\title{
Skalenabhängigkeit von Biodiversität - von der Theorie zur Anwendung
}

\author{
Forschungsbericht \\ zur kumulativen Habilitation
}

\author{
von \\ Dr. rer. nat. habil. Jürgen Dengler
}

Fachbereich Biologie der Universität Hamburg 2012 
Habilitation beantragt: 30.11.2011

Hochschulöffentlicher Vortrag: 10.01.2012

Habilitation im Fach Vegetations- und Makroöologie: 24.04.2012

Forschungsbericht elektronisch veröffentlich: 20.06.2012 


\section{Inhaltsverzeichnis}

1 Einleitung

1.1 Wissenschaftliche Relevanz von Biodiversität ................................................................................... 6

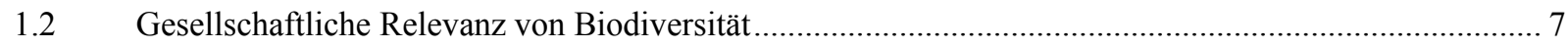

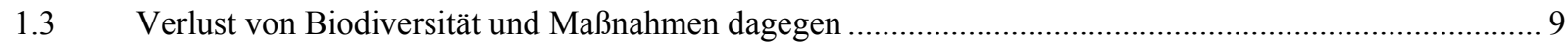

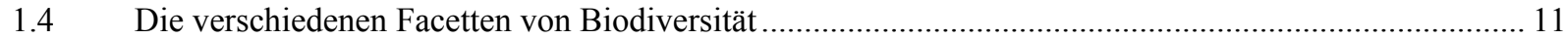

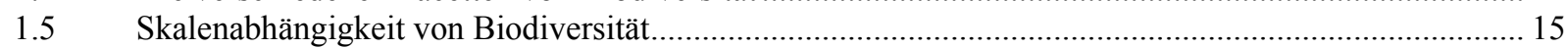

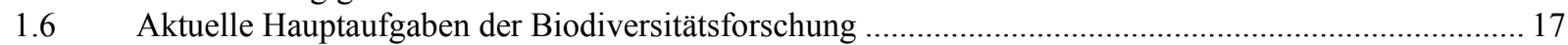

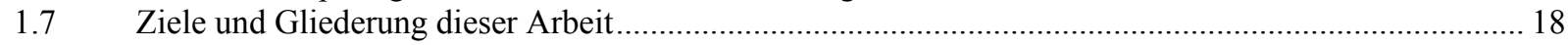

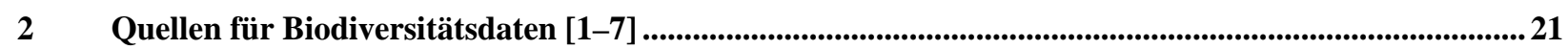

2.1 Methoden zur Erhebung und zum Monitoring von Biodiversität ...................................................... 21

2.2 Vegetationsdatenbanken als Quelle von Biodiversitätsdaten................................................................. 24

3 Theorie, Simulationen und Analysemethoden zur Skalenabhängigkeit von Biodiversität [8-13].......... 29

3.1 Artenzahl-Areal-Beziehungen: Analysemethoden und generelles Konzept ..........................................2 29

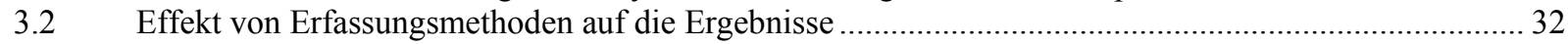

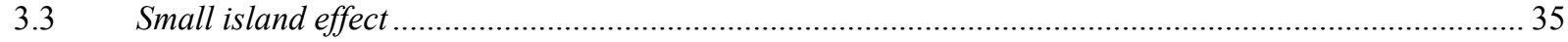

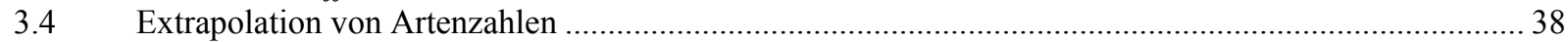

3.5 Auswirkungen der Skalenabhängigkeit von Biodiversität auf Anwendungen ............................................. 39

$4 \quad$ Artendiversität auf verschiedenen Skalenebenen [14-25]............................................................................ 41

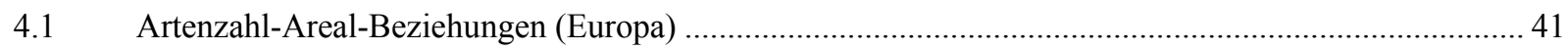

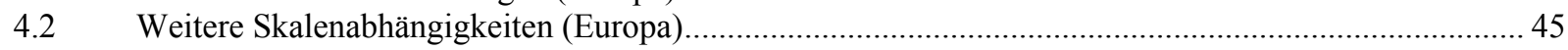

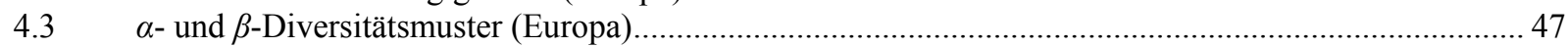

4.4 Abhängigkeit der Artendiversität von der Umwelt (Europa) ................................................................... 49

4.5 Abhängigkeit der Artendiversität von der menschlichen Nutzung (Europa) ...........................................53

4.6 Großräumige Biodiversitätsmuster im südlichen Afrika...................................................................5 57

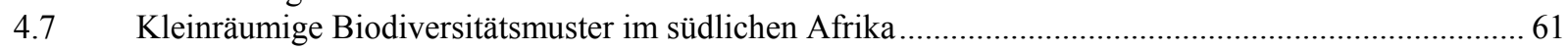

5 Analyse und Diversität von Pflanzengesellschaften [26-38] .......................................................................67

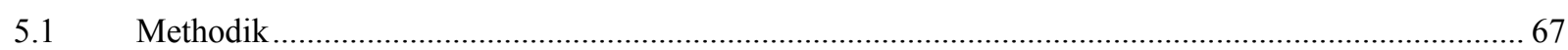

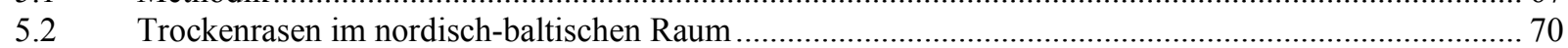

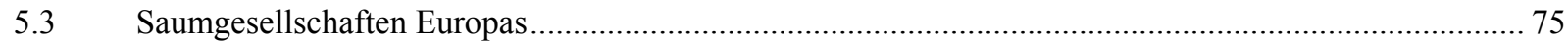

5.4 Montan-subalpine Hochstaudengesellschaften Europas …………………………………………….... 79

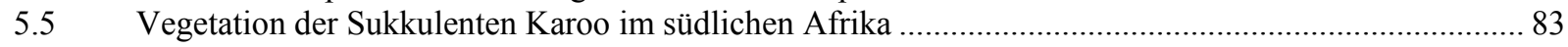

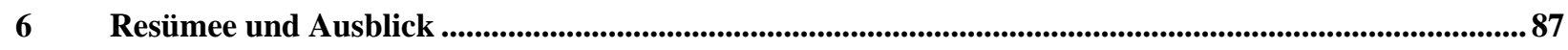

$7 \quad$ Danksagung ................................................................................................................................................ 91

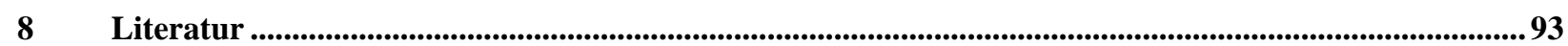

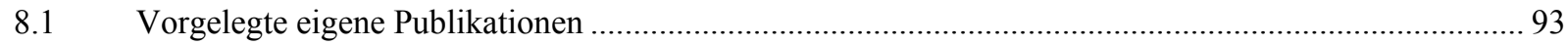

8.2 Alle zitierten Quellen in alphabetischer Reihenfolge.......................................................................... 95

I Curriculum Vitae................................................................................................................................. 115

II Erläuterung des eigenen Anteils an den vorgelegten Publikationen ........................................................119

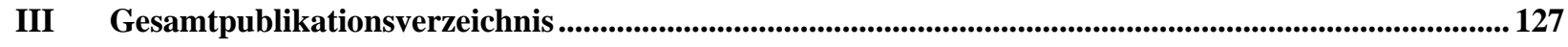

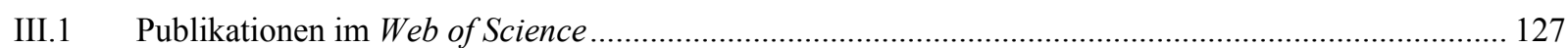

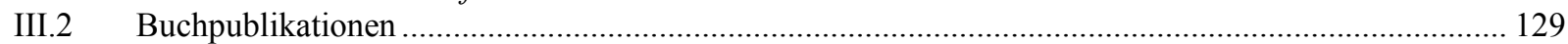

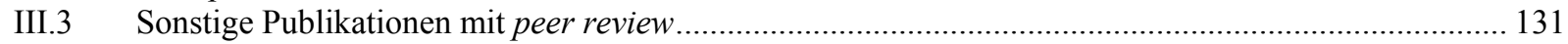

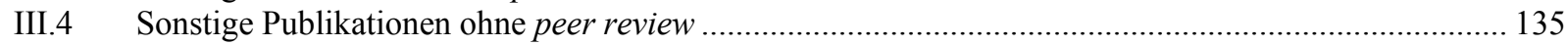

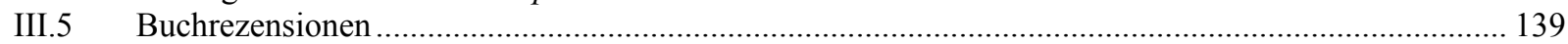

IV Verzeichnis von Lehrveranstaltungen an Universitäten .............................................................................. 141

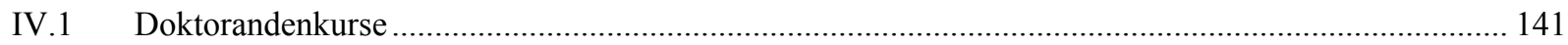

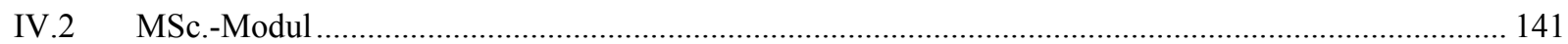

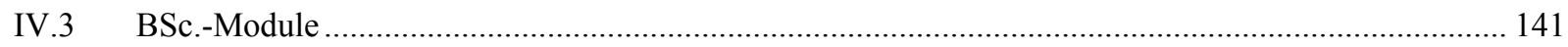

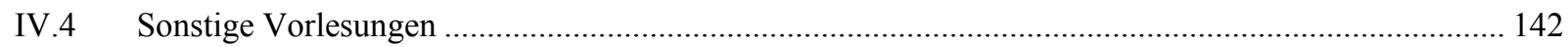

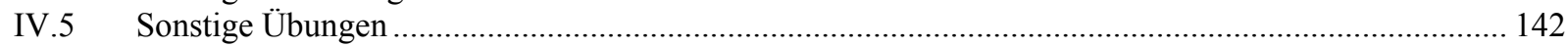




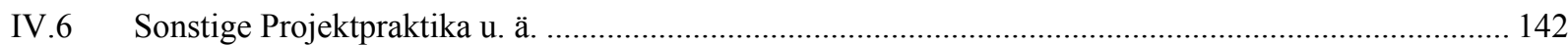

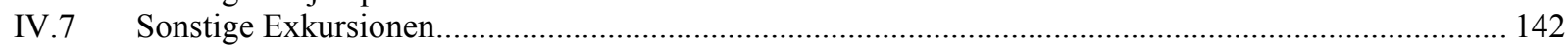

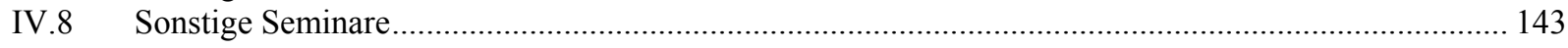

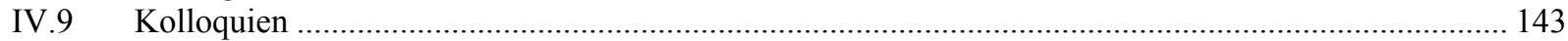

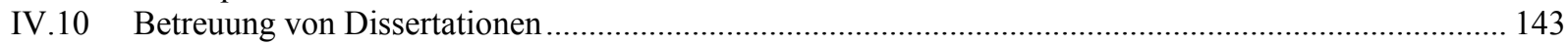

IV.11 Betreuung von Diplom-, Staatsexamens-, Bachelor- und Semesterarbeiten.................................... 143

\section{Anhang I: Kopien der vorgelegten Publikationen, 1. Teil}

Publikationen zu Kapitel 2: Quellen für Biodiversitätsdaten [Publikationen 1-7]

Publikationen zu Kapitel 3: Theorie, Simulationen und Analysemethoden zur Skalenabhängigkeit von Biodiversität [Publikationen 8-13]

Publikationen zu Kapitel 4: Artendiversität auf verschiedenen Skalenebenen [Publikationen 14-25]

\section{Anhang II: Kopien der vorgelegten Publikationen, 2. Teil}

Publikationen zu Kapitel 5: Analyse und Diversität von Pflanzengesellschaften [Publikationen 26-38]

CD-ROM mit den gedruckten Beilagen, Online Supplementary Materials u. ä. der vorgelegten Publikationen sowie farbigen pdf's des Forschungsberichtes und der beiden Anhänge 


\section{Einleitung}

Biodiversität ist ein recht junges Konzept in der Biologie. Als biological diversity (biologische Vielfalt) scheint es erstmals in den 1950er Jahren gebraucht worden zu sein (Magurran 2004). Die kontrahierte Form biodiversity wurde offensichtlich 1985 von W. G. Rosen kreiert (siehe Harper \& Hawksworth 1995) und erschien 1988 erstmals in einer wissenschaftlichen Publikation (Wilson 1988a; deutsch: Wilson 1992). Große Prominenz sowohl in der wissenschaftlichen als auch in der politischen Öffentlichkeit erlangte der Biodiversitäts-Begriff 1992 durch die Convention on Biological Diversity (CBD; deutsch: Übereinkommen über die biologische Vielfalt; United Nations 1992), ein in Rio de Janeiro ausgehandeltes internationales Abkommen, das mittlerweile 168 Staaten der Welt unterzeichnet haben. Seither hat die Anzahl der wissenschaftlichen Publikationen zu Biodiversität sprunghaft zugenommen und steigt noch weiter an (Abb. 1a). Inzwischen sind 42.718 wissenschaftliche Veröffentlichungen $\mathrm{zu}$ diesem Thema im Web of Science erschienen (http://apps.webofknowledge.com; Abfrage am 16.10.2011 mit TOPIC = biodiversity OR "biological diversity"). Anders als in vielen anderen Naturwissenschaften behalten die Ergebnisse der Biodiversitätsforschung offensichtlich ihre Aktualität über lange Zeiträume, wie die seit fast zwanzig Jahren weitgehend konstanten Zitationsraten der BiodiversitätsArtikel aus dem CBD-Jahr 1992 zeigen (Abb. 1b).
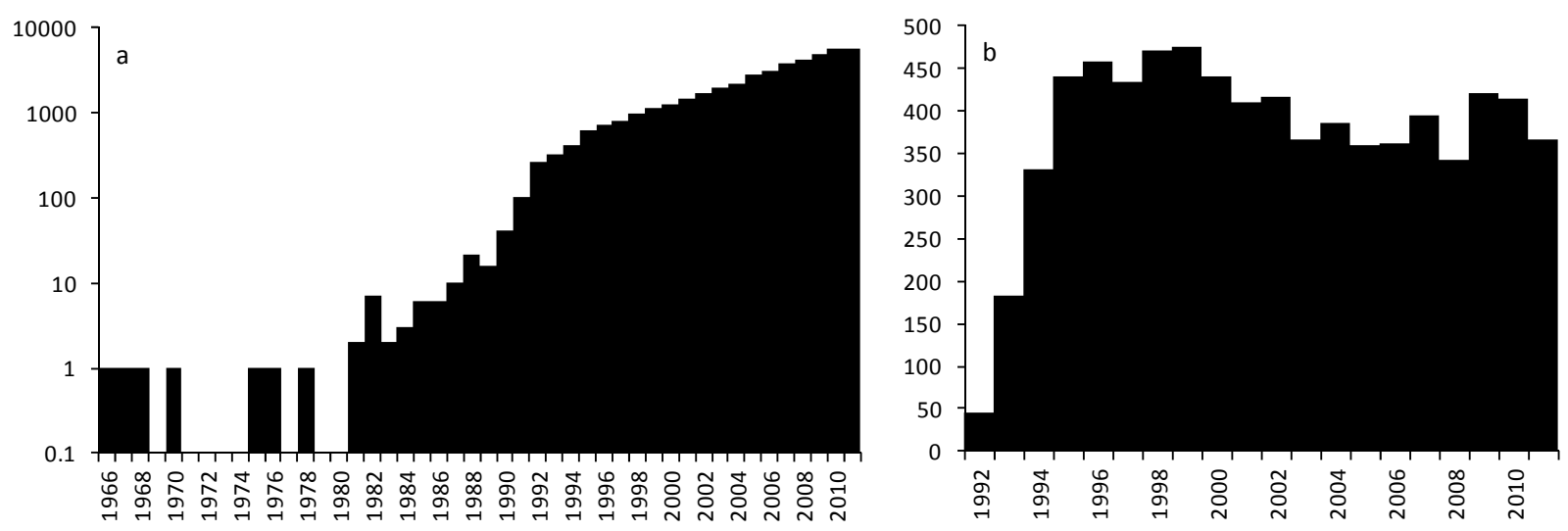

Abb. 1: Publikationen zum Thema Biodiversität im Web of Science (Abfrage am 16.10.2011 mit TOPIC = biodiversity OR "biological diversity"). Teilabbildung a gibt die Anzahl der Veröffentlichungen pro Kalenderjahr in logarithmischer Skalierung an. Im Jahr 1966 gab es erstmals eine Publikation mit dem Begriff Biodiversität im Titel, im Abstract oder in den Keywords, während es 2010 bereits 5589 waren. Teilabbildung b illustriert den zeitlichen Verlauf der Zitierung am Beispiel der im Jahr 1992 veröffentlichten 260 Artikel. In beiden Fällen wurde für das Jahr 2011 der zum Zeitpunkt der Abfrage vorliegende Wert durch Multiplikation mit 4/3 näherungsweise auf das Gesamtjahr extrapoliert. 
Während es viele im Detail unterschiedliche Definitionen des Begriffes Biodiversität gibt (Gaston \& Spicer 2004), ist eine der meist gebrauchten und zugleich umfassendsten jene aus der CBD (United Nations 1992):

"Biological diversity" means the variability among living organisms from all sources including, inter alia, terrestrial, marine and other aquatic ecosystems and the ecological complexes of which they are part: this includes diversity within species, between species and of ecosystems.

Biodiversität als Konzept hat deshalb eine so große Relevanz, weil dieser Begriff einerseits fundamentale Fragen des wissenschaftlichen Verständnisses der biologischen Systeme der Erde zusammenfasst und andererseits der globale Verlust an Biodiversität so große Ausmaße angenommen hat, dass weitgehender Konsens der politischen Akteure herrscht, dass BiodiversitätsSchutz heute ein wichtiges Politikziel ist. Entsprechend haben die Vereinten Nationen die Jahre 2011-2020 als United Nations Decade on Biodiversity ausgerufen (Resolution 65/161; siehe http://www.cbd.int/2011-2020/).

\subsection{Wissenschaftliche Relevanz von Biodiversität}

Die große Vielfalt der Lebewesen auf der Erde hat Forscher seit jeher interessiert und es ist eine der fundamentalsten Fragen der Wissenschaft, wie viele Arten es auf der Erde gibt (Mora et al. 2011). Dabei werden von Taxonomen weiterhin pro Jahr rund 15.000-20.000 Arten neu beschrieben (Chapman 2009, May 2010). Trotz zahlreicher globaler taxonomischer Datenbanken (z. B. Catalogue of Life, www.catalogueoflife.org oder International Plant Name Index, www.ipni.org) ist unklar, wie viele rezente Arten bereits wissenschaftlich beschriebenen wurden; die Schätzungen reichen von etwa 1,244 Millionen (Mora et al. 2011) bis 1,900 Millionen (Chapman 2009). Über die Zahl der tatsächlich vorkommenden rezenten Arten divergieren die Meinungen in der Wissenschaft sogar zwischen etwa 3 Millionen und über 100 Millionen (May 2010), wobei methodisch recht gut abgesicherte Hochrechnungen aus jüngster Zeit 8,7 Millionen (Mora et al. 2011) bzw. 11,3 Millionen (Chapman 2009) prognostizieren. Die Zahl der im Laufe der Erdgeschichte entstandenen und inzwischen größtenteils wieder ausgestorbenen Arten dürfte noch einmal um den Faktor 20-100 höher sein (May 1994, Raup 1994). Mit Darwins Evolutionstheorie (Darwin 1859) stand erstmals ein theoretischer Ansatz zur Verfügung, der die wesentlichen Aspekte der Entstehung dieser Vielfalt überzeugend erklären kann. Moderne Sequenziertechniken in Kombination mit geeigneten statistischen Verfahren erlauben es inzwischen, den 
tatsächlichen Evolutionsverlauf hochauflösend zu rekonstruieren (z. B. Ciccarelli et al. 2006, Qiu et al. 2006, AGP 2009).

Während die Evolutionsforschung zu erklären versucht, wann und wie die vielen unterschiedlichen Taxa entstanden sind, gehört die Beschreibung der ungleichen Verteilung von Biodiversität auf der Erdoberfläche und die Erklärung der zugrunde liegenden Mechanismen zum Kern der Ökologie (Ricklefs \& Schluter 1993, Rosenzweig 1995, Hubbell 2001, Gaston \& Spicer 2004). Hierbei beschäftigt sich die Synökologie (community ecology) und im Bereich der Pflanzen spezifisch die Vegetationsökologie mit kleinräumigen Biodiversitätsmustern in Abhängigkeit von Umweltparametern wie Bodenverhältnissen (z. B. Pärtel 2002, Schuster \& Diekmann 2003), Landnutzung (z. B. Dupré \& Diekmann 2001, Weibull et al. 2003), Störungsregime (z. B. Connell 1978, Collins et al. 1995), räumlicher Heterogenität (z. B. Tilman 1994, Lundholm 2009), Landschaftsstruktur und -geschichte (z. B. Fahrig 2003, Helm et al. 2006) sowie dem regionalen Artenpool (z. B. Eriksson 1993, Dupré et al. 2002). Dagegen sind die sich auf großen Skalenebenen ergebenden Biodiversitätsmuster (z. B. Barthlott et al. 2005) Gegenstand der relativ jungen Teildisziplin Makroökologie (Brown \& Maurer 1989, Brown 1995, Gaston \& Blackburn 2000, Blackburn \& Gaston 2003). Während es unzählige Hypothesen in der Ökologie gibt, die zu erklären versuchen, warum unter bestimmten Umweltbedingungen eine bestimmte Biodiversität auftritt (Reviews z. B. in Grace 1999, Pausas \& Austin 2001, Willig et al. 2003), ist es eine der großen Herausforderungen der Ökologie, diese Einzelansätze zu einer kohärenten Gesamttheorie zusammenzuführen (z. B. Grime 1973, Palmer 1994, Grace 1999, Hubbell 2001, Whittaker et al. 2001, Ricklefs 2004).

Biodiversitätsforschung versucht, aktuelle und historische Gründe gemeinsam zur Erklärung der auftretenden Biodiversitätsmuster heranzuziehen, und bringt damit die vormals getrennten Disziplinen Evolutionsbiologie und Ökologie zusammen. Letztlich geht es um die Frage, warum eine so große Zahl unterschiedlicher Organismenarten entstanden ist und wie es viele davon schaffen, am gleichen Ort von den gleichen fundamentalen Ressourcen zu leben, ohne dass das „Konkurrenz-Ausschluss-Prinzip“ (Review in den Boer 1986, siehe auch Palmer 1994) erkennbar greift.

\subsection{Gesellschaftliche Relevanz von Biodiversität}

Warum sollte sich die Menschheit insgesamt für Biodiversität interessieren und sich über ihren gravierenden Rückgang Sorgen machen? Nach M. Soulé (zitiert in Groom 2006) ist das Aussterben einer Art die unumkehrbarste und tragischste aller Umweltkalamitäten. Die vielen Gründe, warum Biodiversität wertvoll ist und ihre Erhaltung daher wichtig, lassen sich in zwei große 
Gruppen gliedern, intrinsische Werte (weitgehend identisch mit non-use values = nicht nutzungsbezogenen Werten) und utilitaristische Werte (direct- and indirect-use values $=$ Werte durch direkte und indirekte Nutzung), wobei die Grenzziehung je nach Autor etwas divergiert (Gaston \& Spicer 2004, Callicott 2006, Edwards-Jones 2006). Ob Arten ein intrinsischer Wert zugewiesen wird, hängt vom philosophisch-religiösen Weltbild jedes einzelnen ab, doch ist die Ansicht, dass Biodiversität einen Wert an sich hat, in vielen Religionen und Gesellschaften tief verwurzelt (Gaston \& Spicer 2004, Callicott 2006) und findet sich entsprechend auch in vielen nationalen und internationalen Gesetzen und Konventionen zum Biodiversitätsschutz, etwa in der Präambel zur CDB (United Nations 1992). Im weiteren Sinne gehören auch der Vermächtnis-Wert (bequest value) und psycho-spirituale Werte wie ästhetisches Empfinden (die meisten Menschen finden Vielfalt schöner als Eintönigkeit), religiöse Ehrfurcht und wissenschaftliche Erkenntnis zu den nicht-nutzungsbezogenen Werten (Gaston \& Spicer 2004, Callicott 2006, Edwards-Jones 2006).

Die Werte von Biodiversität durch direkten Nutzen (oft auch als biodiversity goods bezeichnet) beziehen sich auf die Nutzung von Arten als Nahrungsmittel, als Medikamente, als Brennstoffe oder als Werkstoffe, sowie die Wertschöpfung aus biodiversen Landschaften im (Öko-) Tourismus (Gaston \& Spicer 2004, Callicott 2006). Während etwa 12.500 Arten der Samenpflanzen als essbar gelten, wurden bislang nur rund 200 domestiziert, von denen wiederum ganze 12 für über $75 \%$ der menschlichen Ernährung verantwortlich sind (Gaston \& Spicer 2004). Hier kann die Nutzung weiterer Wildarten, insbesondere naher Verwandter von derzeitigen Kulturpflanzen die Sicherstellung der menschlichen Ernährung trotz wachsender Weltbevölkerung, anthropogenem Klimawandel oder möglichen Ausbrüchen von Krankheits- und Schädlingskalamitäten gewährleisten (Iltis 1988, Plotkin 1988, Gaston \& Spicer 2004). Auch heute noch basieren die Medikamente, die in den Industrieländern genutzt werden, größtenteils auf Sekundärstoffwechselprodukten von Organismen (Farnsworth 1988, Dobson 1995, Gaston \& Spicer 2004). Ungefähr jede 125. Pflanzenart, die im Detail darauf untersucht wurde, führte zu der Entdeckung eines wichtigen pharmakologischen Wirkstoffes, wobei bislang aber nur ein verschwindend kleiner Anteil der Pflanzen- und andere Organismenarten systematisch „gescreent“ wurde (Dobson 1995). Hier liegen riesige Potenziale, wie verschiedene Beispiele aus jüngerer Zeit zeigen, etwa Paclitaxel (Taxol), ein erst 1971 entdeckter Wirkstoff aus der Rinde von Taxus brevifolia, der inzwischen Grundlage für das meistverkaufte Krebsmedikament ist (vgl. Gaston \& Spicer 2004).

In jüngerer Zeit besonders ins Blickfeld gerückt ist der indirekte Nutzen von Biodiversität, oft als biodiversity services (oder ecosystem services) bezeichnet (Naeem et al. 1994, Mooney et al. 
1995, Chapin et al. 2000, Tilman 2000, Loreau et al. 2001, Gaston \& Spicer 2004, Hooper et al. 2005, Callicott 2006, Naeem et al. 2009). Hier wird argumentiert, dass eine höhere Biodiversität in den meisten Fällen mit einer besseren Funktionalität von Ökosystemen einhergeht, die wiederum zu einer Bereitstellung von Dienstleistungen für den Menschen, wie Regulation von Klima und Wasserhaushalt, erhöhter Biomasseproduktion und größerer Stabilität von Ökosystemen sowie Kontrolle von Schadorganismen in und Bereitstellung von Bestäubern für Agrarflächen einhergeht. Verschiedene experimentelle Studien legen nahe, dass höhere Biodiversität in der Regel zu höherer Biomasseproduktion (z. B. Tilman et al. 1996, Hector et al. 1999, Hooper et al. 2005), aber auch zu höherer Stabilität von Ökosystemeigenschaften führt (z. B. Naem \& Li 1997, Hooper et al. 2005, Haddad et al. 2011). Ein weiteres wichtiges Beispiel ist der Umstand, dass in agrarischen Systemen die Qualität und Quantität der Bestäubung und damit der Ertrag des jeweiligen landwirtschaftlichen Produktes maßgeblich positiv durch die Diversität der Bestäubergemeinschaften beeinflusst wird (Review in Klein et al. 2009). Generell gibt es eine hohe Übereinstimmung zwischen den Erdregionen mit besonders hoher und zugleich bedrohter Biodiversität und einem überdurchschnittlichen Wert der Ökosystemdienstleistungen (Turner et al. 2007).

\subsection{Verlust von Biodiversität und Maßnahmen dagegen}

Das Aussterben von Arten ist wie ihre Neuentstehung ein normaler, letztlich sogar notwendiger Bestandteil der Evolution. Arten und höhere Taxa haben alle eine begrenzte Lebensdauer, die sich in eine mittlere Aussterberate umrechnen lässt (Raup \& Sepkoski 1982, Marshall 1988, Raup 1994, Pimm et al. 1995). Je nach Großgruppe betrug die mittlere Lebensdauer von Arten in der Fossilgeschichte zwischen 1 und 25 Millionen Jahren, entsprechend einer Aussterberate von etwa 0,04-1 pro 1 Million Art-Jahre (May 1994, Raup 1994, Pimm et al. 1995, Gaston \& Spicer 2004). Dieser relativ konstante Arten-Turnover bei insgesamt ansteigendem Trend war in der Erdgeschichte durch fünf große Massen-Aussterbe-Ereignisse (mass extinction events) unterbrochen, bei denen jeweils innerhalb paläontologisch kurzen Zeiträumen 75-96 \% der zuvor lebenden Arten ausstarben, was einer Erhöhung der Aussterberate um ein bis zwei Größenordnungen gegenüber dem Normalen entspricht (Raup 1994, Groom 2006).

Mit dem Erscheinen des modernen Menschen auf dem Planeten hat sich das Bild grundlegend geändert. So ist für den Zeitraum seit 1600 das Aussterben von 816 Arten (389 Wirbellose, 337 Wirbeltiere und 90 Pflanzen) dokumentiert (Hunter 2002). Da nur bei zuvor wissenschaftlich beschriebenen Taxa und auch hier nur bei guter Datenlage ein Verlust überhaupt registriert werden kann, ist evident, dass die tatsächlichen Aussterberaten in den letzten vier Jahrhunderten 
erheblich darüber gelegen haben müssen. Aber schon steinzeitliche Menschen haben große Zahlen von Großsäugern und Vögeln zum Aussterben gebracht; allein durch die Besiedlung der pazifischen Inseln durch den Menschen sind Schätzungen zu Folge über 2000 Vogelarten in wenigen Jahrtausenden ausgestorben (Pimm et al. 1995, Gaston \& Spicer 2004). Extrapoliert man diese Befunde auf die schlechter untersuchten bzw. noch gar nicht beschriebenen Taxa, lässt sich schlussfolgern, dass die Aussterberaten in den letzten Jahrhunderten bei etwa 10-100 pro 1 Million Art-Jahre lagen, also bereits hundertfach über dem evolutiv Normalen (Pimm et al. 1995, World Resources Institute 2005). Während die historischen Verluste überwiegend durch direkte Verfolgung/Nutzung von Arten oder durch anthropogene Invasionen exotischer Arten bedingt waren, dürften aktuell und im kommenden Jahrhundert die Faktoren Landnutzung (d. h. Verlust von natürlichen oder naturnahen Lebensräumen), Klimawandel und Eutrophierung die stärksten Verursacher von Biodiversitätsverlust sein (Sala et al. 2000; siehe auch Wilson 1988b, Vitousek et al. 1997, Thomas et al. 2004). Die zu erwartenden Aussterberaten im 21. Jahrhundert werden nach verschiedenen Schätzungen um den Faktor 300-300.000 über der Hintergrundrate liegen, wenn sich an der globalen Umweltpolitik nichts Grundsätzliches ändert (Wilson 1988b, Pimm et al. 1995, World Resources Institute 2005, Groom 2006, Pereira et al. 2010), so dass sich mit Fug und Recht von einem sechsten Massen-Aussterbe-Ereignis sprechen lässt (Groom 2006), das in seiner Geschwindigkeit alle bisherigen weit in den Schatten stellt.

Das Wissen über den rapiden Rückgang der globalen Biodiversität zusammen mit der sich verbreitenden Erkenntnis über die Bedeutung von Biodiversität für die Menschheit fand schon lange seinen Ausdruck in nationalen Naturschutzgesetzen und -programmen, Roten Listen gefährdeter Arten, Pflanzengesellschaften und Biotope in vielen Ländern und auch global (v. a. IUCN 2011; vgl. Review von Mace et al. 2008) sowie in einzelnen internationalen Verträgen zum Biodiversitätsschutz, etwa der Convention on International Trade in Endangered Species of Wild Fauna and Flora (CITES) von 1973 (siehe http://www.cites.org/) und der Convention on Wetlands (Ramsar-Konvention) von 1971 (siehe http://www.ramsar.org/). Doch erst mit der Verabschiedung der CBD in Rio de Janeiro (United Nations 1992) erhielt der Biodiversitätsschutz einen wirklich einflussreichen Platz in der internationalen Politik. In Folge der CDB fanden bislang zehn Vertragsstaatenkonferenz (Conferences of the Parties $=$ COPs) statt und 173 Staaten haben mittlerweile den von der CBD geforderten National Biodiversity Strategies and Action Plan (NBSAP) verabschiedet (siehe http://www.cbd.int/). Im Jahr 2002 auf der sechsten Konferenz der CBD-Vertragsstaaten vereinbarten diese, „bis 2010 eine signifikante Reduktion der gegenwärtigen Rate des Biodiversitätsverlustes zu erzielen“ [Übersetzung durch den Verfasser] (siehe Balmford et al. 2005). Dieses Ziel wurde jedoch klar verfehlt, wie inzwischen viele 
Wissenschaftler, aber auch das Sekretariat der CBD selbst konzidieren (z. B. Butchart et al. 2010, Mace et al. 2010, Secretariat of the Convention on Biological Diversity 2010). Nach dieser Enttäuschung werden inzwischen neue Ziele und Wege für den globalen Biodiversitätsschutz für das Jahrzehnt bis 2020 formuliert (z. B. Mace et al. 2010, Rands et al. 2010), die auch Eingang in den auf der zehnten Vertragsstaatenkonferenz beschlossenen Strategic Plan for Biodiversity 2011-2020 fanden (siehe http://www.cbd.int/decision/cop/?id=12268).

\subsection{Die verschiedenen Facetten von Biodiversität}

In den bisherigen Abschnitten wurde Biodiversität in vielen Fällen gleichgesetzt mit der Anzahl von Arten innerhalb einer räumlich, zeitlich und/oder taxonomisch abgegrenzten Einheit, etwa die rezenten Gefäßpflanzen Hamburgs. Man verwendet hierfür meist den Fachbegriff Artenreichtum (species richness), wobei das deutsche Wort Artenzahl anders als das englische species number keine Doppeldeutigkeit hat und daher synonym verwendet werden kann. Die weit verbreitete Verwendung des Artenreichtums als Kenngröße in der Biodiversitätsforschung liegt laut Gaston \& Spicer (2004) an (a) seiner praktischen Anwendbarkeit, (b) der Datenverfügbarkeit, (c) seiner Eignung als Surrogat für andere Biodiversitätsaspekte und (d) seiner Übereinstimmung mit dem außerhalb der Wissenschaft vorherrschenden Verständnis von Biodiversität. Artenreichtum ist das älteste und intuitivste Maß von Biodiversität (Magurran 2004) und damit quasi die gemeinsame Währung (,common currency“) in der Biodiversitätsforschung (Gaston \& Spicer 2004), obwohl es zahlreiche weitere Arten gibt, Biodiversität zu quantifizieren.

Bereits die CBD stellt aber klar, dass mit Biodiversität viel mehr gemeint ist als nur die Artenvielfalt, indem sie die drei Komplexitätsebenen (a) genetische Diversität (diversity within species), (b) taxonomische bzw. organismische Diversität (diversity between species) und (c) ökologische Diversität (diversity of ecosystems) benennt (United Nations 1992). Diese drei Ebenen werden auch von den meisten anderen Biodiversitätsforschern als grundlegend anerkannt (Harper \& Hawksworth 1995, van der Maarel 1997, Gaston \& Spicer 2004). Dabei umfasst jede dieser Ebenen wiederum verschiedene Elemente, die organismische Diversität etwa die Diversität von Arten, Gattungen oder Ordnungen (Gaston \& Spicer 2004).

Der Diversitätsbegriff im Allgemeinen, wie der Biodiversitätsbegriff im Speziellen, umfassen zwei Komponenten: (a) die Anzahl von Entitäten und (b) die Unterschiede zwischen diesen (Gaston \& Spicer 2004; siehe auch Purvis \& Hector 2000, Magurran 2004). Unterschiede zwischen den Entitäten (auf einer der drei Komplexitätsebenen) können wiederum als bloße Häufigkeitsunterschiede oder aber als Unterschiede in den Eigenschaften (siehe dazu den folgenden Absatz) analysiert werden. Betrachtet man die Häufigkeit (von Arten innerhalb einer Probe, von 
Allelen innerhalb einer Population o. ä.), lässt sich der Anzahl (richness) die Gleichverteilung (evenness) als im Optimalfall unabhängigen (orthogonalen) Eigenschaften gegenüberstellen (Purvis \& Hector 2000, Magurran 2004, Olszewski 2004). Eine Stichprobe wird immer dann als besonders divers angesehen, wenn die darin enthaltenen Entitäten näherungsweise gleich häufig sind. Es gibt zahlreiche verschiedene Ansätze, evenness zu berechnen, wobei viele nicht die geforderte Unabhängigkeit von der richness erfüllen (Smith \& Wilson 1996). Diversitätsmaße, die die richness-Komponente und die evenness-Komponente in einer einzigen Größe zu vereinigen suchen, werden gewöhnlich als „Diversitätsindizes“ bezeichnet (Magurran 2004), etwa der Shannon-Index, der Simpson-Index oder der Berger-Parker-Index (van der Maarel 1997, Magurran 2004). Viele dieser Diversitätsindizes stehen in einem mathematischen Zusammenhang, der sogenannten Hill's family (van der Maarel 1997) mit

$$
N_{a}=\sum_{i=1}^{S}\left(p_{i}{ }^{a}\right)^{1 /(1-a)},
$$

wobei die $N_{a}$ die verschiedenen Indizes darstellen, $S$ die Artenzahl (oder Anzahl sonstiger Entitäten) und $p_{i}$ die relative Abundanz oder Deckung der Art $i$ sind und $a$ einen Wert zwischen $-\infty$ und $+\infty$ annehmen kann, entsprechend einem Gradienten von einer starken Gewichtung der seltenen zu einer starken Gewichtung der häufigen Arten (Hill 1973, van der Maarel 1997). Die Artenzahl selbst entspricht $N_{0}$, während $N_{1}$ die exponentielle Form des Shannon-Index $H^{\prime}$ ist, also exp $\left(H^{\prime}\right)$. Die verschiedenen Realisationen von $N_{a}$ reflektieren damit zugleich unterschiedliche Gewichtungen der richness- und der evenness-Komponente von Biodiversität.

Allen bisher besprochenen Ansätzen zur Quantifizierung von Biodiversität ist gemein, dass sie alle Arten als gleichwertig ansehen. Sie entsprechen daher dem, was Jürgens et al. (im Druck) innerhalb ihrer durch die Arbeit von Noss (1990) inspirierten vier Dimensionen von Biodiversität als compositional dimension (Dimension der Zusammensetzung) bezeichnen. Die drei weiteren von ihnen angeführten Dimensionen sind (b) Struktur, (c) Funktion und (d) Evolution. Insbesondere die beiden letztgenannten Diversitätsaspekte haben in jüngerer Zeit große Beachtung gefunden. So wurden zahlreiche Vorschläge zur Berechnung funktionaler Diversität (FD; z. B. Petchey \& Gaston 2002, 2006, Ricotta 2005b, Villéger et al. 2008, de Bello et al. 2009) und phylogenetischer Diversität (PD; z. B. Faith 1992, Harper \& Hawksworth 1994, van der Maarel 1997, Hardy \& Senterre 2007, Schweiger et al. 2008, Cadotte et al. 2010) gemacht und diskutiert. Allen gemeinsam ist, dass hier die Arten bei der Diversitätsberechnung umso stärker gewichtet werden, je unähnlicher sie sich funktional sind oder je weiter sie im Stammbaum isoliert voneinander stehen. Anders als bei FD und PD sind die Versuche, die verbleibende der vier Di- 
mensionen von Jürgens et al. (im Druck), die strukturelle Diversität, zu quantifizieren bislang selten und uneinheitlich (z. B. Carranza et al. 1998, Zehm et al. 2003).

Schließlich kann Biodiversität auf drei räumlichen Hierarchieebenen betrachtet werden. Whittaker $(1960,1972)$ hat das Konzept von $\alpha-, \beta$-, und $\gamma$-Diversität ursprünglich für den Artenreichtum eingeführt, doch finden analoge Ansätze inzwischen auch für viele andere Facetten von Biodiversität Anwendung, etwa für funktionale Diversität (de Bello et al. 2009) oder phylogenetische Diversität (z. B. Hardy \& Senterre 2007, Graham \& Fine 2008). Später wurden teilweise noch weitere Hierarchieebenen zu diesem Schema hinzugefügt (siehe Tab. 1; vgl. Magurran 2004). Alle Hierarchieebenen lassen sich dabei auf zwei fundamental verschiedene Kategorien aufteilen (Whittaker 1960, 1972, Magurran 2004, Jurasinski et al. 2009; siehe Tab. 1): Erfassungsdiversität (inventory diversity) und Differenzierungsdiversität (differentiation diversity). Da die einzelnen Hierarchieebenen zwar verbal umschrieben, aber nicht präzise definiert sind und zudem in der Literatur für extrem unterschiedliche räumliche Ausdehnungen verwendet wurden (vgl. Jursasinski et al. 2009), sollte man m. E. die drei Begriffe $\alpha-, \beta$-, und $\gamma$-Diversität universell verwenden, jedoch jeweils die betrachteten Skalen benennen. Damit sind $\alpha$ - und $\gamma$-Diversität die mit dem gleichen Diversitätsindex gemessenen Erfassungsdiversitäten (z. B. Artenreichtum) auf zwei unterschiedlichen, meist räumlichen Skalen, während die $\beta$-Diversität die „Verknüpfung“ dieser beiden Ebenen darstellt, oft als turnover bezeichnet (vgl. aber Vellend 2001, der die undifferenzierte Verwendung des Begriffes turnover kritisiert). Wie schon von Whittaker (1960, 1972) herausgestellt und von verschiedenen aktuellen Reviews (Vellend 2001, Gaston et al. 2007, Jurasinski et al. 2009, Tuomisto 2010a, 2010b, Anderson et al. 2011) zusammengefasst, gibt es verschiedene Möglichkeiten, die Differenzierung zu messen. Im Kern lassen sich Maße, die nur auf die Anzahl (und ggf. Häufigkeitsverteilung) der Elemente abheben (proportional diversity bei Jurasinski et al. 2009 bzw. classical $\beta$ diversity metrics bei Anderson et al. 2011) solchen Maßen gegenüberstellen, die zusätzlich die Artidentitäten berücksichtigen (differentiation diversity [s. str.] bei Jurasinski et al. 2009 bzw. multivariate measures of $\beta$ diversity bei Anderson et al. 2011). Innerhalb jeder dieser beiden Gruppen gibt es wiederum verschiedene Ansätze bzw. Indizes, für die proportional diversity etwa das additive und das multiplikative Verfahren sowie die Steigung der Artenzahl-Areal-Beziehung (Jurasinski et al. 2009, Anderson et al. 2011). Über die Vor- und Nachteile der einzelnen Ansätze gibt es eine intensive akademische Debatte (z. B. Ricotta 2005a, Crist \& Veech 2006, Jost 2007, Tuomisto 2010a), aber ich stimme der Schlussfolgerung von Gaston et al. (2007) und Anderson et al. (2011) zu, dass es nicht ein einziges, bestes $\beta$-Diversitätsmaß gibt, sondern dass verschiedene Maße unterschiedliche relevante Aspekte beleuchten und so erst gemeinsam ein vollständiges Bild ergeben. Verschiedene 
Ansätze zur Bestimmung der $\beta$-Diversität erlauben es auch gleichermaßen, eine hierarchische Partitionierung der großräumigen Diversität ( $\gamma$-Diversität) in $\alpha$ - und $\beta$-Komponenten vorzunehmen (Loreau 2000, Ricotta 2005a, Crist \& Veech 2006, Hardy \& Senterre 2007, Jost 2007, de Bello et al. 2009, Tuomisto 2010a, Anderson et al. 2011).

Tab. 1: Räumliche Hierarchieebenen von Biodiversität, die Zusammenhänge zwischen diesen und ihre Zugehörigkeit zu den zwei Kategorien Erfassungsdiversität und Differenzierungsdiversität (nach Magurran 2004). Die drei meistgebrauchten und zugleich vom Verfasser als sinnvoll erachteten Ebenen sind fett hervorgehoben.

\begin{tabular}{lcc}
\hline Räumliche Hierarchieebene & Erfassungsdiversität & Differenzierungssdiversität \\
\hline Innerhalb einer Stichprobe & Punktdiversität & \\
Zwischen Stichproben, innerhalb eines Habitates & & Musterdiversität \\
Innerhalb eines Habitates & $\boldsymbol{\alpha}$-Diversität & $\boldsymbol{\beta}$-Diversität \\
Zwischen Habitaten, innerhalb einer Landschaft & & $\boldsymbol{\gamma}$-Diversität \\
Innerhalb einer Landschaft & & $\delta$-Diversität \\
$\begin{array}{l}\text { Zwischen Landschaften, innerhalb einer biogeografischen } \\
\text { Provinz }\end{array}$ & & \\
Innerhalb einer biogeografischen Provinz & & \\
\hline
\end{tabular}

Diese kurze Einführung in die Vielfalt unterschiedlichster Quantifizierungen von Biodiversität zeigt, dass es essenziell ist, bei jeder Aussage zum Thema Biodiversität genau zu spezifizieren, auf welche Komplexitätsebene, welches Element, welche Komponente, welche Dimension und welche räumliche Hierarchieebene man sich bezieht. Ich fasse diese unterschiedlichen Kombinationen dieser Kategorisierungen hier unter dem Begriff Facetten der Biodiversität zusammen. Dabei lassen sich die einzelnen Kategorien nahezu beliebig kombinieren. So ist etwa die in der Vegetationsökologie häufig verwendete Kenngröße „Artenzahl in einer Vegetationsaufnahme“ die Kombination von organismischer Diversität (Komplexitätsebene) bezogen auf Arten (Element), gemessen als Anzahl der Elemente (Komponente) bezüglich der Zusammensetzung (Dimension) auf der $\alpha$-Ebene (räumliche Hierarchieebene). Dagegen analysieren de Bello et al. (2009) ebenfalls auf der organismischen Komplexitätsebene und für das Element Arten Diversitätsindizes, die die beiden Komponenten Anzahl und Häufigkeit verbinden, für den Aspekt funktionelle Diversität sowohl auf der $\alpha$ - und $\beta$-Hierarchieebene.

Schließlich gibt es für die meisten Kombinationen der genannten Facetten von Biodiversität wiederum mehrere Möglichkeiten, sie zu quantifizieren - einige wurden oben angedeutet. Angesichts dieser theoretischen und praktischen Vielschichtigkeit des Diversitätsbegriffes, scheint es mir nicht zielführend, ein einziges „echtes“ Diversitätsmaß vielen ,unechten“ gegenüberzustel- 
len (wie es Tuomisto 2010b für die $\beta$-Diversität vorschlägt), noch den Begriff „Diversitätsindex“ auf jene Maße zu verengen, die auf der Ebene der Artendiversität richness- und evennessKomponenten vereinen (z. B. Magurran 2004). Stattdessen gebrauche ich in dieser Arbeit den Begriff Biodiversitätsindex (auch Biodiversitätsparameter oder Biodiversitätsmaß) für jede Form der Quantifizierung einer Facette von Biodiversität. Damit ist der Artenreichtum einer Probe oder eines Gebietes der am häufigsten gebrauchte und m. E. zugleich auch wichtigste Biodiversitätsindex.

\subsection{Skalenabhängigkeit von Biodiversität}

Unter Skalenabhängigkeit von Biodiversität verstehe ich das Phänomen, dass die meisten Biodiversitätsindizes aber auch deren Beziehungen zu Umweltparametern nicht universell sind, sondern sich je nach betrachteter Skalenebene unterscheiden. Folgende drei Skalierungsaspekte sind hier bedeutsam: (1) räumliche Skala; (2) zeitliche Skala und (3) taxonomische oder phylogenetische Skala. Die räumliche Skala ist der bei Weitem bestuntersuchte Bereich, doch zeigen einige neuere Untersuchungen, dass es entsprechende Skalenabhängigkeiten auch für die zeitliche Skala (d. h. Zeitraum; z. B. Adler et al. 2005, Magurran 2007, White 2007, McGlinn \& Palmer 2009) und die taxonomische Skala (d. h. taxonomische Hierarchieebene; z. B. Storch \& Šizling 2008, Mora et al. 2011) gibt und dort oft sehr ähnliche Gesetzmäßigkeiten wie für die räumlich Skala gelten. Im Folgenden möchte ich mich aber auf die räumliche Skala beschränken, wobei hier wiederum mehrere Aspekte unterschieden werden müssen. Nach Scheiner et al. $(2000,2011)$ gibt es vier Komponenten der (räumlichen) Skala, sampling unit, grain, focus und extent, wobei für die meisten Betrachtungen grain und extent die entscheidenden sind. Grain (Korngröße) meint die Größe einer Fläche, für die direkt erhobene Biodiversitätsdaten (z. B. Artenzahlen) vorliegen; extent (Ausdehnung) dagegen die Gesamtfläche des Areals (Polygons), in dem die einzelnen Probeflächen verteilt liegen.

Ihre offensichtlichste Manifestation erfährt die Skalenabhängigkeit von Biodiversität in den Artenzahl-Areal-Beziehungen (species-area relationships, SARs). Dass die Artenzahl mit zunehmender Flächengröße zunimmt, ist ein lang bekanntes Phänomen (Arrhenius 1920, Gleason 1922) und diese Artenzahl-Areal-Beziehungen gelten manchen Ökologen als eines der wenigen fundamentalen Gesetze in der Ökologie (Schoener 1976, Lawton 1999, Lomolino 2000). Die Frage, wie sich diese Artenzahlzunahme mit zunehmender Flächengröße mathematisch am besten beschreiben lässt, ist dagegen bis heute Gegenstand eines intensiven wissenschaftlichen Diskurses (z. B. Preston 1962, Connor \& McCoy 1979, Tjörve 2003, 2009, Stiles \& Scheiner 2007, Guilhaumon et al. 2008). Die mathematische Beschreibung von Artenzahl-Areal-Beziehungen 
hat dabei große theoretische und praktische Bedeutung. Zum einen sagen verschiedene ökologische Theorien unterschiedliche Kurvenverläufe für SARs voraus, womit umgekehrt die für reale Daten ermittelten Funktionen Rückschlüsse auf deren Richtigkeit der Theorien bzw. zumindest deren relative Bedeutung im jeweiligen Fall zulassen (z. B. Hubbell 2001, Stark et al. 2006). Weiterhin sind Artenzahl-Areal-Beziehungen erforderlich, um Artenzahlen aus verschiedenen Untersuchungen auf eine einheitliche Flächengröße (grain) zu „standardisieren“, etwa um Vergleiche durchzuführen oder Artendichtekarten zu erstellen (z. B. Barthlott et al. 2005). Große Bedeutung haben Artenzahl-Areal-Beziehungen (und ähnliche Skalenabhängigkeiten) bei der Extrapolation von Artendiversität auf große Flächen, deren vollständige Inventarisierung schwer oder oft gar nicht möglich wäre (Colwell \& Coddington 1994, He \& Legendre 1996, Plotkin et al. 2000, Scheiner et al. 2011). Schließlich gibt es zahlreiche Anwendungen im Naturschutz, insbesondere die Identifizierung von Biodiversitäts-Hotspots (z. B. Veech 2000, Fattorini 2006, Guilhaumon et al. 2008), die optimale Schutzgebietsplanung (z. B. Desmet \& Cowling 2004), die Vorhersage des Artenschwundes in Folge von Habitatverlusten (z. B. Pimm et al. 1995, Connor \& McCoy 2001, Ulrich 2005, Koh \& Ghazoul 2010) oder Klimawandel (z. B. Thomas et al. 2004) und die Beurteilung anthropogener Einflüsse auf Biodiversität (z. B. Tittensor et al. 2007).

Neben der bloßen Existenz von Artenzahl-Areal-Beziehungen zeigt sich Skalenabhängigkeit von Biodiversität noch in vielen anderen Bereichen. Diese Skalenabhängigkeit, ihre theoretische Erklärung, ihre praktische Nutzung und die Handhabung der von ihr verursachten Probleme sind ein zentrales Thema der Ökologie, wie zwei hochrangige Sammelbände aus jüngerer Zeit belegen (Peterson \& Parker 1998, Storch et al. 2007a). So sind sowohl die den Artenzahl-ArealBeziehungen zu Grunde liegenden Prozesse skalenabhängig (z. B. Shmida \& Wilson 1985, Cornell \& Karlson 1997, Turner \& Tjørve 2005, Field et al. 2009) als auch in manchen Fällen der $z$ Wert, welcher die relative Zunahme der Artenzahl bei einer Flächenvergrößerung beschreibt (z. B. Crawley \& Harral 2001, Drakare et al. 2006, Dolnik \& Breuer 2008). Weiterhin beeinflusst die räumliche Skala zahlreiche andere Aspekte von Biodiversität, etwa Gemeinschaftsmuster (co-occurrence patterns), wie man sie in der Synökologie mittels Ordinationen darstellt und analysiert (z. B. Otýpková \& Chytrý 2006), den Arten-Turnover entlang von Umweltgradienten (z. B. Reed et al. 1993, Grytnes et al. 2008), räumliche Muster von Diversitätsparametern (z. B. Kallimanis et al. 2008) oder Abundanzverteilungen von Arten (z. B. Gleason 1929, Dengler 2003). $\mathrm{Zu}$ den meisten genannten Aspekten gibt es bislang nur wenige regionale Fallstudien, die Allgemeingültigkeit der publizierten Beziehungen ist daher fraglich, andere wurden bislang praktisch gar nicht untersucht, etwa der Effekt unterschiedlicher Korngrößen (z. B. Aufnahmeflächen) auf die als universell geltenden latitudinalen und altitudinalen Diversitätsgradienten 
(z. B. Rosenzweig 1995) oder auf die Vorkommenshäufigkeit („Stetigkeit“) von Arten. Letztere ist relevant, wenn man den Einfluss von anderen Umweltparametern (außer der Fläche) auf das Vorkommen bestimmter Arten bestimmen will oder um diagnostische Arten für die Klassifikation von Lebensgemeinschaften zu ermitteln.

In jüngster Zeit gibt es vermehrt Bemühungen, die unterschiedlichsten Typen von Biodiversitätsmustern (etwa Artenzahl-Areal- und Artenzahl-Zeit-Beziehungen, Artenhäufigkeitsverteilungen) und ihrer Skalenabhängigkeit in einem gemeinsamen theoretischen Rahmen zusammenzuführen, wozu einerseits die neutrale Biodiversitätstheorie und andererseits die makroökologische Perspektive maßgeblich beigetragen haben (Ritchie \& Olff 1999, Gaston \& Blackburn 2000, Hubbell 2001, Storch \& Gaston 2004, Harte 2007, Storch et al. 2008, Ritchie 2010). Da sich einige dieser Muster als (nahezu) allgemeingültig erwiesen haben, besteht die Hoffnung, dass dies auch für die zu Grunde liegenden Prozesse gilt (Storch et al. 2007b). In vielen der Ansätze stehen fraktale Eigenschaften der Natur im Zentrum (z. B. Lennon et al. 2007, Palmer 2007, Storch et al. 2008, Ritchie 2010). In jüngster Zeit haben Nekola \& Brown (2007) herausgestellt, dass in anderen komplexen Systemen (Klima, Ökonomie, Zitationen von Publikationen) sehr ähnliche Diversitätsmuster (sie sprechen ganz allgemein von distributions of wealth) vorkommen, ihre Ergründung mithin zum fundamentalen Verständnis komplexer Systeme und ihrer emergenten Eigenschaften ganz allgemein beiträgt.

\subsection{Aktuelle Hauptaufgaben der Biodiversitätsforschung}

Auf der Basis des Geschilderten sehe ich aktuell die folgenden sieben Hauptaufgaben für die Biodiversitätsforschung, die von der Theorie und Datenerhebung über Analysen und die dafür notwendigen Techniken bis hin zur Anwendung reichen. Dabei vereint die Biodiversitätsforschung Ansätze aus der Phylogenie, Ökologie, Biogeografie und Systemtheorie:

a. Wissenschaftliche Beschreibung aller rezenten Arten von Organismen, einschließlich Aufklärung ihrer phylogenetischen Beziehungen.

b. Verfügbarmachen von umfassenden Daten über alle Arten von Organismen, insbesondere ihrer Verbreitung und wesentlicher funktioneller Merkmale.

c. Klärung der terminologischen und methodischen Fragen, die mit der Vielzahl beschriebener Biodiversitätsparameter und Biodiversitätsmuster verknüpft sind, einschließlich der mathematischen Zusammenhänge zwischen diesen.

d. Umfassende Beschreibung und mechanistische Erklärung, wie verschiedene Facetten der Biodiversität von Umweltparametern (einschließlich Landnutzung und historischen Parametern) abhängen. 
e. Umfassende Beschreibung und mechanistische Erklärung, wie Biodiversität in ihren verschiedenen Facetten Einfluss auf Ökosystemfunktionen und damit letztlich auf ökosystemare Güter und Dienstleistungen für uns Menschen nimmt.

f. Entwicklung von Monitoringsystemen, die eine Beurteilung der künftigen Biodiversitätsentwicklung unter sich abzeichnenden Entwicklungen wie Klima- und Landnutzungswandel erlaubt.

g. Entwicklung von Maßnahmen, die einen effektiven und umfassenden Biodiversitätsschutz ermöglichen, zugleich aber mit den menschlichen Bedürfnissen vereinbar sind.

\subsection{Ziele und Gliederung dieser Arbeit}

$\mathrm{Zu}$ den im vorstehenden Abschnitt herausgearbeiteten Kernzielen der Biodiversitätsforschung leisten die Publikationen, auf denen diese Habilitationsschrift beruht, in vier großen Themenblöcken (s. u.) wichtige Beiträge. Dabei lege ich auf den bislang immer noch oft vernachlässigten Aspekt der Skalenabhängigkeit besonderes Gewicht. Zugleich versuche ich in jedem dieser vier Bereiche den Bogen von theoretisch-methodischen Überlegungen über die empirische Forschung bis hin zu praktischen Anwendungen zu spannen. Die als Beispiele gewählten Artengruppen gehören meist zu den Organismenreichen der Pflanzen (Gefäßpflanzen und Moose) und Pilze (Flechten), vereinzelt habe ich aber auch Tiere betrachtet (Asseln als makroökologisches Modellsystem, Großherbivoren als Weidetiere). Die vier Themenblöcke entsprechen den Kapiteln 2-5 dieser Arbeit:

Kapitel 2 (Quellen für Biodiversitätsdaten) beschäftigt sich mit zwei wesentlichen Aspekten der Aufgaben b und f. Ein erste Gruppe der dort referierten Publikationen präsentiert Kriterien für optimale Verfahren für die standardisierte Erhebung und das Monitoring von Biodiversitätsdaten im Gelände unter Einbeziehung des Skalenaspektes und diskutiert Erfahrungen mit deren praktischer Implementierung. Eine zweite Gruppe wirft ein Schlaglicht auf den großen, bislang aber völlig unzureichend genutzten Fundus hochwertiger Biodiversitätsdaten in Vegetationsdatenbanken und beleuchtet Chancen, aber auch Probleme ihrer Nutzung.

Kapitel 3 (Theorie, Simulationen und Analysemethoden zur Skalenabhängigkeit von Biodiversität) thematisiert innerhalb der Aufgabe c verschiedene Aspekte von Skalenabhängigkeit, insbesondere von Artenzahl-Areal-Beziehungen und ähnlichen Gesetzmäßigkeiten. Hier zeige ich, dass es in diesem Bereich bedingt durch problematische Methoden oder unpräzise Terminologie nicht selten zu falschen Schlussfolgerungen bis hin zum Aufstellen unbegründeter Theorien kommt. Als Konsequenz daraus erarbeite ich eine klare Terminologie der verschiedenen Phänomene und entwickle geeignete statistische Analysemethoden. Die relevanten mathe- 
matischen Zusammenhänge beleuchte ich mittels theoretischer Überlegungen, Simulationen und Fallstudien.

Kapitel 4 (Artendiversität auf verschiedenen Skalenebenen) widmet sich Aufgabe d bezogen auf die organismische Komplexitätsebene. Die einzelnen Abschnitte thematisieren die Abhängigkeit der Artendiversität von der räumlichen Skala, von den betrachteten Biodiversitätsfacetten, von der abiotischen Umwelt und von der menschlichen Nutzung. Dies geschieht anhand von Beispielen aus unterschiedlichen Ökosystemen in Europa und Afrika. In einigen Arbeiten gehe ich hier auch auf Aufgabe g ein.

Kapitel 5 (Analyse und Diversität von Pflanzengesellschaften) schließlich widmet sich ebenfalls der Aufgabe d, aber bezogen auf die ökologische Komplexitätsebene (hier: Pflanzengesellschaften). Es geht um adäquate Verfahren zur Beschreibung der Vielfalt von Pflanzengesellschaften, wobei ich zunächst einige methodische Aspekte beleuchte. Die folgenden Abschnitte beschäftigen sich dann mit der großräumigen Vielfalt und Klassifikation von Trockenrasen, Saum- und Hochstaudengesellschaften in verschiedenen Teilen Europas sowie der Vegetation der Sukkulenten Karoo in Südafrika.

Das abschließende Kapitel 6 führt die Erkenntnis aus den vier Themenblöcken dann zu einem Resümee zusammen und gibt einen kurzen Ausblick auf zu klärende offene Fragen.

Im folgenden Text sind die eigenen Publikationen, welche Bestandteil dieser kumulativen Habilitationsschrift sind, mit einer fortlaufenden, in eckige Klammern gesetzten und fett gedruckten Nummer hinter dem Zitat gekennzeichnet, also beispielsweise „Dengler (2009b) [1]“. Im Literaturverzeichnis finden sich diese Arbeiten in Abschnitt 8.1 unter ihrer Nummer und aus Gründen der Benutzerfreundlichkeit zusätzlich alphabetisch eingereiht im vollständigen Quellenverzeichnis in Abschnitt 8.2. Diese genannten eigenen Arbeiten sind in den beiden Anhängen in eben dieser Reihenfolge abgedruckt. 


\section{Quellen für Biodiversitätsdaten [1-7]}

\subsection{Methoden zur Erhebung und zum Monitoring von Biodiversität}

Vor allem in Folge der Verpflichtungen aus der Convention on Biological Diversity (CBD) zur Dokumentation der Entwicklung der Biodiversität auf ihrem jeweiligen Territorium haben die meisten Staaten der Erde inzwischen Biodiversitätsmonitoringprogramme aufgelegt (siehe http://www.cbd.int/nbsap/; vgl. Dengler 2009b [1]). Daneben gibt es auch vielerlei andere lokale bis globale Monitoringansätze für Biodiversität, die teils erst jüngst etabliert wurden, wie die DFG Biodiversitäts-Exploratorien (Fischer et al. 2010), die teils aber auch auf Datenreihen zurückgreifen können, die lange vor der CBD beginnen, wie einige der in ILTER (International Long Term Ecological Research, Kim 2006) zusammengeschlossenen Projekte (Review in Jürgens et al. im Druck [2]).

Bei den zahlreichen unterschiedlichen Aktivitäten zur Ersterfassung und zum langfristigen Monitoring unterschiedlichster Aspekte von Biodiversität fällt auf, dass vielfach ad-hocLösungen implementiert werden, ohne dass die Komplexität von Biodiversitätsdaten wirklich durchdrungen und nach einer optimalen Lösung gesucht worden wäre. Die Übersicht von Bischoff \& Dröschmeister (2000) über die Biodiversitätsmonitoring-Programme in europäischen Ländern zeigt, dass selbst in diesem mit wissenschaftlicher Kompetenz und finanziellen Resourcen gut ausgestatteten Kontinent die angewandten oder vorgeschlagenen Monitoringverfahren moderne Erkenntnisse der Biodiversitätsforschung, insbesondere zur Skalenabhängigkeit von Biodiversität, oftmals ignorieren und zudem von Land zu Land so unterschiedlich sind, dass kontinentweite Auswertungen kaum möglich erscheinen. Oftmals wird in Biodiversitätsmonitoringprogrammen aber gar nicht die Biodiversität und ihre Veränderung selbst erfasst, sondern andere, leichter und kostengünstiger zu erfassende Parameter, die in einem nicht bekannten $\mathrm{Zu}-$ sammenhang zur tatsächlichen Biodiversität stehen. So misst nur ein einziger der im Hinblick auf das CBD-Ziel Halting the loss of biodiversity by 2010 für Europa vorgeschlagenen und implementierten sogenannten 26 SEBI 2010-Indikatoren (Streamlining European 2010 Biodiversity Indicators; EEA 2007, Schutyser et al. 2009) tatsächlich Veränderungen in der Biodiversität Europas (Indikator 01, Teil a: Populationsindex für weit verbreitete Vogelarten, Teil b: Populationsindex für Tagfalterarten des Grünlandes). Drei weitere Indizes messen indirekt die tatsächliche oder drohenden Veränderungen der Artendiversität von ausgewählten Taxa (Indikator 02: Red List Index; Indikator 03: Species of European interest) und die genetische Diversität von Nutztieren (Indikator 06: Livestock genetic diversity), indem sie Experteneinschätzungen zur Gefährdungsstatus bzw. zum Erhaltungszustand aggregieren. Die übrigen 22 Indikatoren be- 
schäftigen sich nur indirekt mit Biodiversitätsänderungen, indem sie zu quantifizieren versuchen, wie sich Maßnahmen mit mutmaßlich positivem (Schutzgebietsausweisungen) oder negativem (Nährstoffeintrag in Ökosysteme) Effekt auf Biodiversität im Laufe der Zeit auswirken. Man muss also konstatieren, dass es, abgesehen von den Brutvögeln, selbst in Europa de facto kein wirkliches Biodiversitätsmonitoring gibt.

Hier setzen die beiden Publikationen von Dengler (2009b) [1] und Jürgens et al. (im Druck) [2] an, indem sie (a) basierend auf dem aktuellen Stand der Forschung Kriterien für ein zielführendes Biodiversitätsmonitoring formulieren, (b) existierende Monitoringsysteme bezüglich ihrer Stärken und Schwächen evaluieren und (c) einen eigenen Vorschlag basierend auf den beiden ersten Punkten entwickeln und begründen. Das von Dengler (2009b) [1] vorgeschlagene System zielt auf eine standardisierte, skalenübergreifende Erfassungsmethode für botanische Diversität, sowohl für Einmalerfassungen als auch für das Monitoring. Das in Jürgens et al. (im Druck) [2] präsentierte System der BIOTA-Biodiversitätsobservatorien zielt primär auf Monitoring der Biodiversität unterschiedlichster Taxa (Pflanzen, Tiere, Flechten, Mikroorganismen) ebenfalls auf verschiedenen Skalenebenen und gemeinsam mit den relevanten Umweltfaktoren (Boden, Wetter, Landnutzung) und ist damit eingebettet in die Ideen eines Global Earth Observation System of Systems (GEOSS; Scholes et al. 2008). Beide Ansätze sind weltweit in allen terrestrischen und semiterrestrischen Lebensräumen anwendbar. Trotz unabhängiger Entstehung weist der botanische Teil der BIOTA.-Biodiversitätsobservatorien große Ähnlichkeiten mit dem von Dengler (2009b) [1] entwickelten Konzept auf. Wesentliche Aspekte, die beide Ansätze von den meisten anderen bisherigen Erfassungs- und Monitoring-Verfahren absetzen, sind:

1. Parallele Erfassung mehrerer räumlicher Flächengrößen (grain sizes), die sich vorzugsweise jeweils um eine Zehnerpotenz unterscheiden und die zugleich mit den Flächengrößen wichtiger bisheriger Erfassungssysteme wie den Whittaker-Plots und ihren Modifikationen (Shmida 1984, Stohlgren et al. 1995, Peet et al. 1998) sowie in der Pflanzensoziologie häufig gebrauchten oder als Standards vorgeschlagenen Flächengrößen (Chytrý \& Otýpková 2003, Dengler 2003) bestmögliche Übereinstimmung haben (also $1 \mathrm{~m}^{2}, 10 \mathrm{~m}^{2}, 100 \mathrm{~m}^{2}, \ldots$ ). Dieses Vorgehen erlaubt es, (a) Biodiversitätsmuster, ihre Abhängigkeit von Umweltfaktoren und ihre zeitliche Veränderung auf mehreren Skalenebenen zu studieren und (b) Extrapolationen von Artenzahlen auf größere, schwerlich direkt erfassbare Flächen vorzunehmen (siehe Abschnitt 3.4).

2. Die kleineren Flächengrößen werden innerhalb jedes Untersuchungsgebietes repliziert, da die Variabilität von Artenzahlen hier besonders hoch ist (s. a. Dengler 2006 [17]) und daher eine 
Mittelwertbildung sinnvoll ist, um das „Durchschlagen“ stochastischen „Rauschens“ auf die Ergebnisse zu vermeiden (s. a. Dengler \& Boch 2008b [11])

3. Um statistisch belastbare Aussagen zu erlauben, etwa die Bildung unverzerrter Mittelwerte, werden die Serien geschachtelter Aufnahmeflächen (nested plot series) randomisiert (oder zumindest systematisch) innerhalb des Untersuchungsgebietes (extent; z. B. Landschaftsausschnitt) verteilt. Das Gleiche gilt für die Anordnung der replizierten Teilaufnahmeflächen gleicher Größe innerhalb der größten Fläche einer Serie.

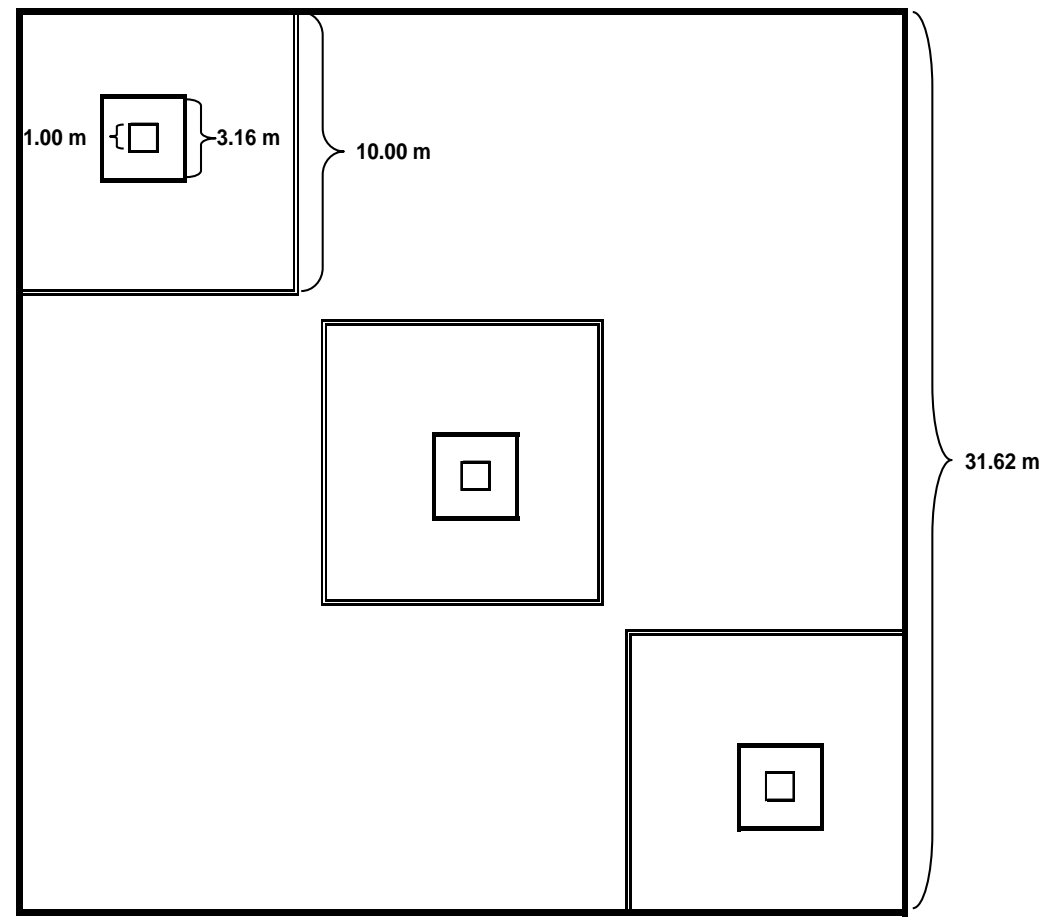

Abb. 2: Beispielhafte Umsetzung des von Dengler (2009b) [1] vorgeschlagenen Designs für die Erfassung und das Monitoring von botanischer Artendiversität. Dargestellt ist eine nested-plot-Serie mit einer größten Aufnahmefläche von $1.000 \mathrm{~m}^{2}$. Jede der drei $1 \mathrm{~m}^{2}$-Flächen beinhaltet noch je eine Teilfläche mit $0,1 \mathrm{~m}^{2}$ und eine mit $0,01 \mathrm{~m}^{2}$ (nicht dargestellt). In den Aufnahmeflächen aller Größen werden komplette Artenlisten aller oberirdisch sichtbaren Pflanzenarten (incl. Moosen, Flechten und Makroalgen) angefertigt, auf einer oder auch mehreren Flächengrößen kann zusätzlich eine Deckungsschätzung der Arten erfolgen. Insgesamt wird eine größere Zahl solcher nested-plot-Serien randomisiert innerhalb des Landschaftsausschnittes angeordnet, dessen Biodiversität es zu charakterisieren gilt. (verändert aus Dengler 2009b [1]). 


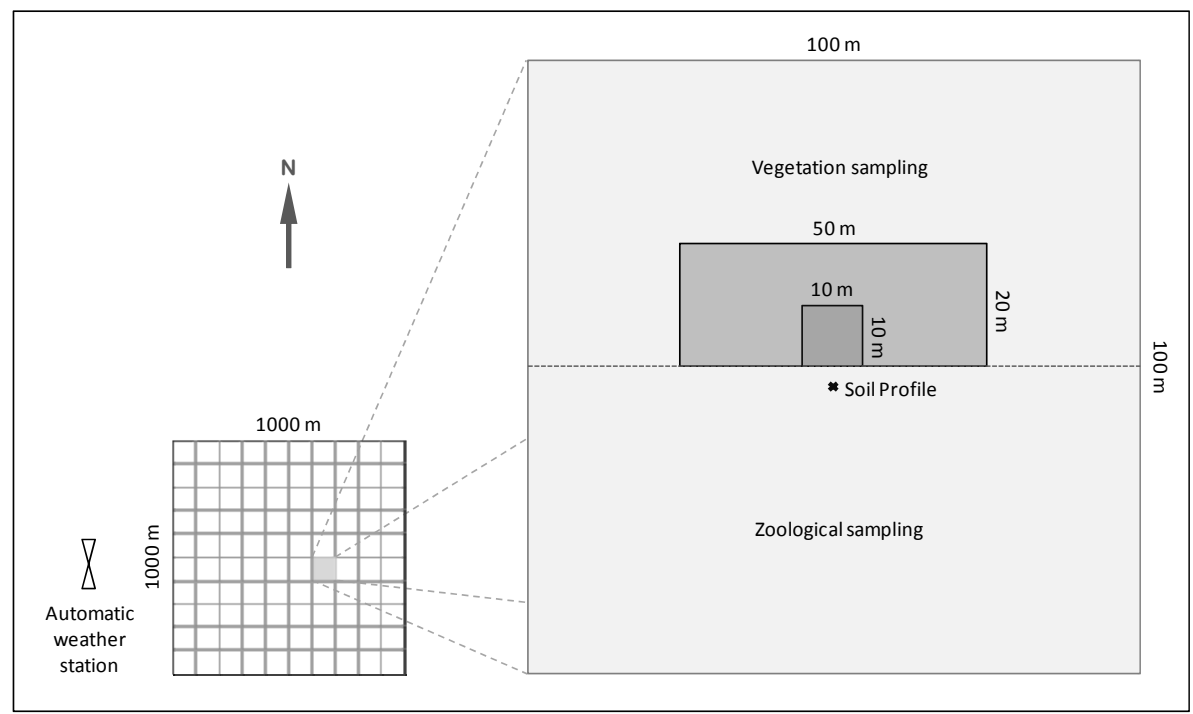

Abb. 3: Schematische Darstellung des Erhebungsdesigns der BIOTA Biodiversitätsobservatorien (aus Jürgens et al. im Druck) [6]. Innerhalb der $1 \mathrm{~km}^{2}$ großen Observatorien werden typischerweise 20 der 100 1-ha-Flächen nach einer mittels eines stratifiziert-randomisierten Verfahren erstellten Rangfolge für die genauere botanische Untersuchung ausgewählt. Auf diesen findet dann das Monitoring der Gefäßpflanzendiversität auf drei geschachtelten Flächen der Größen $100 \mathrm{~m}^{2}, 1000 \mathrm{~m}^{2}$ und $10.000 \mathrm{~m}^{2}$ (= 1 ha) statt. Die anderen Disziplinen (Zoologie, Bodenkunde) können je nach Bedarf und Möglichkeiten eine andere Anzahl von 1-ha-Flächen analysieren, wobei die höchstrangigen Flächen von allen Disziplinen gemeinsam bearbeitet werden.

In Abb. 2 ist eine mögliche Umsetzung des modularen Konzeptes von Dengler (2009b) [1] zur Erfassung von botanischer Artendiversität illustriert. Verschiedene Varianten davon wurden in den vergangenen Jahren in unterschiedlichen Kontexten erfolgreich angewandt (z. B. Allers \& Dengler 2007 [16], Dengler 2005 [15], 2006 [17], Dengler \& Boch 2008b [11]). Abb. 3 zeigt die methodischen Kernaspekte, wie Biodiversitätsmonitoring - nicht nur für Planzen - auf BIOTABiodiversitätsobservatorien erfolgt. Dieses Verfahren blickt inzwischen auf ein Jahrzehnt erfolgreiche Anwendung entlang von drei Großtransekten in Afrika zurück (Jürgens et al. im Druck [2]). Wesentliche auf diesem Ansatz basierende Publikationen aus dem südlichen Afrika sind etwa Haarmeyer et al. (2010b) und Schmiedel et al. (2010) [23].

\subsection{Vegetationsdatenbanken als Quelle von Biodiversitätsdaten}

Ökoinformatik bzw. Biodiversitätsinformatik (ecoinformatics, biodiversity informatics) ist eine noch junge, sich aber dynamisch entwickelnde Disziplin an der Schnittstelle von Ökologie und Informatik (Bisby 2000, Kareiva 2001, Brunt et al. 2002, siehe Review in Dengler et al. 2011a [3]). Während „Bioinformatik“ in den letzten zwei Jahrzehnten zu einem zentralen Pfeiler der Biowissenschaften wurde, setzte sich mehr und mehr eine Verengung des Begriffes auf die An- 
wendung von Ansätzen der Informationstheorie und -technik auf molekularbiologische Daten durch, während Ökoinformatik im tatsächlichen Sinne des Begriffs ein wichtiger Teil der Bioinformatik ist. Eine federführend vom Verfasser in Hamburg organisierte Tagung brachte Anfang 2010 knapp 150 Ökoinformatiker aus aller Welt zusammen, um über den aktuellen Stand der Forschung zu diskutieren. Daraus entstand eine Sonderausgabe des Journal of Vegetation Scien$c e$, welche mit methodischen und konzeptionellen Beiträgen wie auch beispielhaften Analysen den aktuellen Stand der Disziplin aufzeigt und künftige Entwicklungen skizziert, insbesondere unter dem Aspekt möglicher Beiträge der Ökoinformatik zur global change-Debatte (Dengler et al. 2011a [3]).

Ein zentraler Aspekt sind hier die riesigen und bislang nur ansatzweise genutzten Datenmengen in Vegetationsdatenbanken (Ewald 2001, Schaminée et al. 2009, Dengler et al. 2011b [4], Jansen et al. 2011 [5]). Als ein Ergebnis der Ökoinformatik-Tagung in Hamburg wurde unter Federführung des Verfassers eine globale Metadatenbank für Vegetationsdatenbanken gegründet, der Global Index of Vegetation-Plot Databases (GIVD) (Dengler et al. 2011b [3]). Diese Metadatenbank steht inzwischen im Internet jedermann zur Verfügung (www.givd.info) und erfreut sich großer Beliebtheit (über 600.000 Zugriffe seit September 2010). Auf dieser Plattform können Nutzer jetzt anhand unterschiedlicher Kriterien nach Vegetationsdaten suchen, die für ihre jeweilige Fragestellung geeignet sind, und erfahren zu welchen Konditionen diese von den jeweiligen Datenbankbesitzern bereit gestellt werden.

Ende 2010 waren in GIVD insgesamt 132 Datenbanken weltweit registriert, die rund 4,5 Millionen Vegetationsaufnahmen (incl. Zeitreihen und nested plots) von knapp 2,5 Millionen unabhängigen Aufnahmeflächen enthielten (Dengler et al. 2011b [4]). Der GIVD-Initiative ist es damit in kürzester Zeit gelungen, rund $70 \%$ der nach gegenwärtigem Kenntnisstand in elektronischen Datenbanken verfügbaren Vegetationsaufnahmen zu erschließen (Dengler et al. 2011b [4]). Die meisten Länder der Erde sind zumindest mit einzelnen Vegetationsaufnahmen in GIVD vertreten, doch liegt die mit Abstand größte Datendichte in Europa vor, gefolgt von Neuseeland, südlichem Afrika und den USA (Abb. 4). In Dengler et al. (2011b) [4] analysieren wir die Eigenschaften der in GIVD registrierten Vegetationsaufnahmen eingehend, insbesondere auch im Hinblick auf ihre Eignung für ökologische und biodiversitätsbezogene Analysen. Es zeigte sich unter anderem, dass die älteste erfasste Vegetationsaufnahme aus dem Jahr 1864 stammt, wobei Vegetationsaufnahmen in größerer Zahl erst nach dem Zweiten Weltkrieg angefertigt wurden. Immerhin in $17 \%$ bzw. $10 \%$ der erfassten Vegetationsaufnahmen wurden neben Gefäßpflanzen auch Moose bzw. Flechten erfasst. An Umweltdaten ist in 59 \% der Fälle eine Lokalisierung mit einer Genauigkeit von mindestens $1 \mathrm{~km}$ vorhanden, gefolgt von Meereshöhe (46 \%), Hangneigung 
und -exposition (38\%) und Landnutzungsinformationen (25\%). Die verwendeten Flächengrößen variieren erheblich, von weniger als $1 \mathrm{~m}^{2}$ bis zu $10.000 \mathrm{~m}^{2}$ und mehr (Abb. 5), was einerseits Ausdruck der (früher) fehlenden Standardisierung ist (siehe dazu auch Abschnitte 2.1 und 3.5) und anderseits die Notwendigkeit aufzeigt, Methoden $\mathrm{zu}$ entwickeln, die derartige heterogene Daten trotzdem nutzbar machen (z. B. Dengler et al. 2009 [13]).

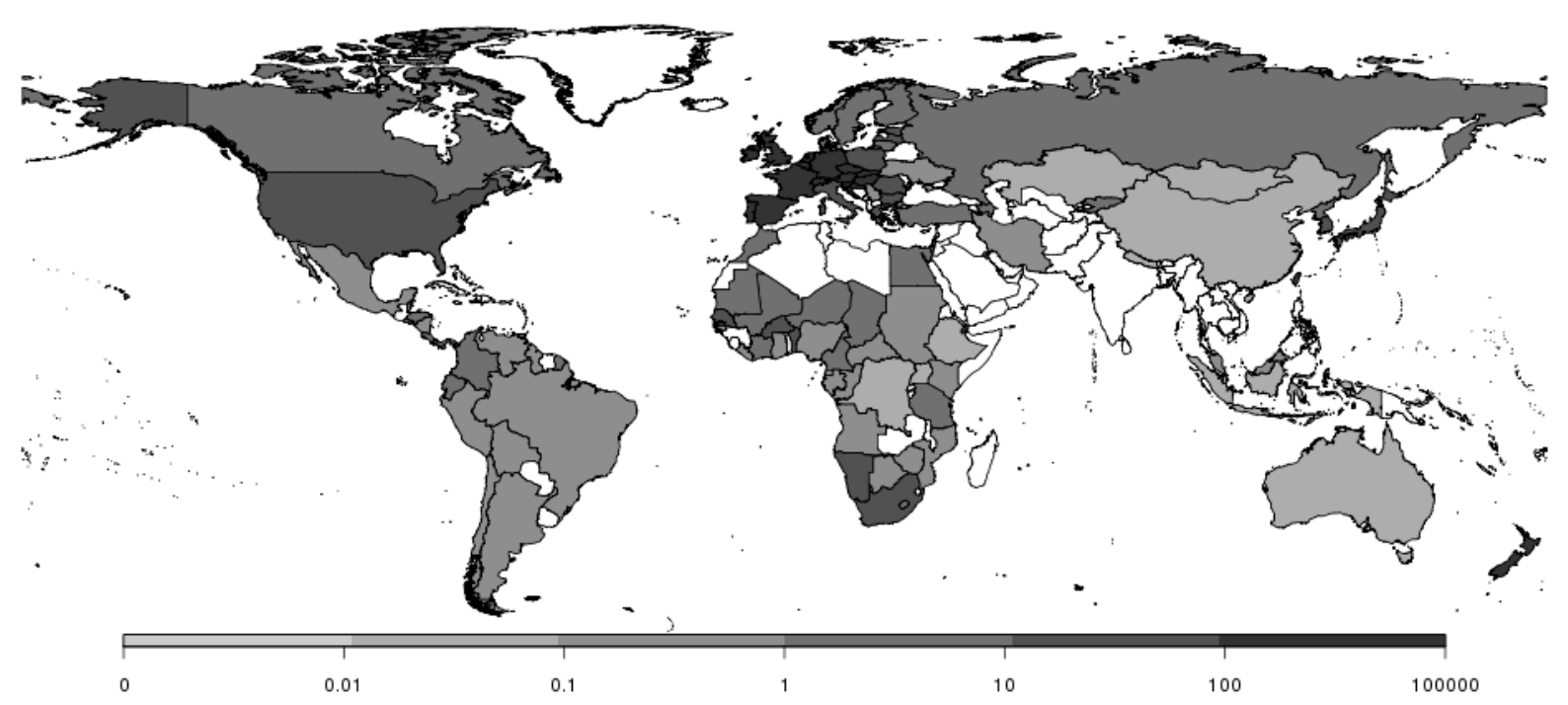

Abb. 4: Globale Verteilung der insgesamt rund 2,5 Millionen unabhängigen Vegetationsaufnahmen, die Ende 2010 in GIVD registriert waren (aus Dengler et al. 2011b [4]). Die Dichte ist als Anzahl von Vegetationsaufnahmen pro $1.000 \mathrm{~km}^{2}$ dargestellt und logarithmisch skaliert.

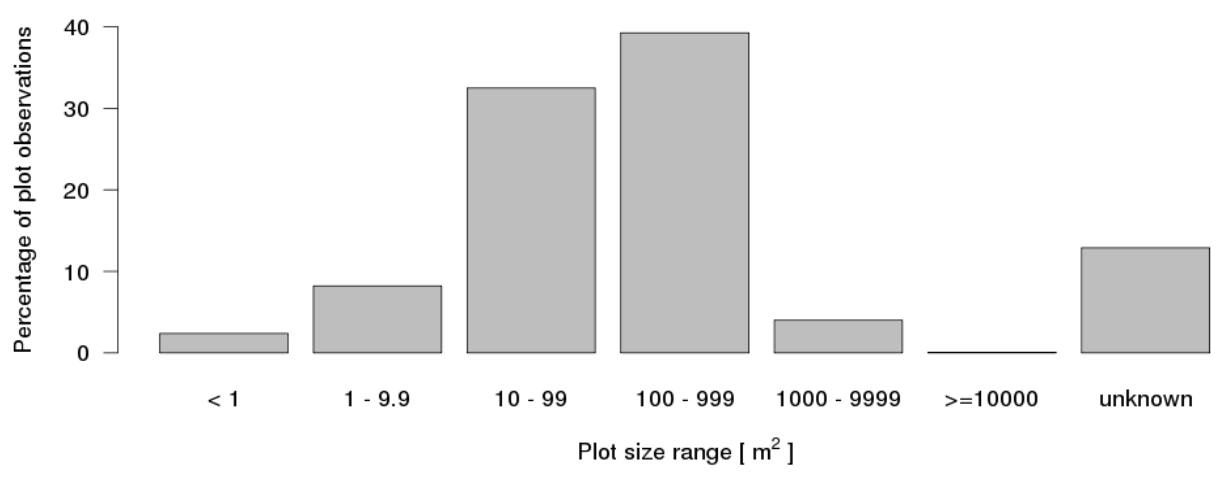

Abb. 5: Globale Verteilung der insgesamt rund 4,5 Millionen Vegetationsaufnahmen (incl. Zeitreihen und nested plots), die Ende 2010 in GIVD registriert waren (aus Dengler et al. 2011b [4]). Auf der Abszisse sind die Flächengrößen der Vegetationsaufnahmen in $\mathrm{m}^{2}$, auf der Ordinate deren relativer Anteil dargestellt.

Von den weltweit in GIVD-Datenbanken registrierten Vegetationsaufnahmen stammen $56 \%$ aus Mitteleuropa (Jansen et al. 2011 [5]). Diese gute Datenlage ist sicherlich der langen und intensiven pflanzensoziologischen Tradition in dieser Region zu verdanken, doch kommt es auch dar- 
auf an, dass frühzeitig Persönlichkeiten mit Organisationstalent und großen integrativen Fähigkeiten eine nationale Datenbanklösung angestoßen haben. So haben die kleinen Niederlande dank der Initiative von Schaminée et al. (1995 ff.) heute eine einzige große Datenbank mit rund 600.000 Vegetationsaufnahmen, während das größere Deutschland seinen Bestand von geschätzten 2,3 Millionen Vegetationsaufnahmen (Schaminée et al. 2009) bislang zu weniger als $10 \%$ digitalisiert hat und die verfügbaren digitalen Aufnahmen - auch in Folge des föderalen Systems - auf zahlreiche kleine und mittelgroße Datenbank verstreut sind (derzeit 180.000 Aufnahmen in 27 Datenbanken; Jansen et al. 2011 [5]).

Der Wert der mit GIVD erschlossenen Vegetationsdaten liegt zunächst an ihrer großen Fülle. Wenn man von durchschnittlich 20 Arten pro Vegetationsaufnahme ausgeht, entsprechen 2,5 Millionen Vegetationsaufnahmen rund 50 Millionen Nachweisen von Pflanzenarten in Raum und Zeit, was ein enormer zusätzlicher Datenfundus verglichen mit den derzeit von GBIF, der Global Biodiversity Information Facility (http://data.gbif.org/), erschlossenen rund 217 Millionen Datensätzen für alle Taxa (einschließlich Tieren, Pilzen, Mikroorganismen) ist (Dengler et al. 2011b [4]). Gegenüber den reinen Vorkommensdaten in GBIF haben die von GIVD erschlossenen Vegetationsdaten erhebliche Vorteile, da sie zusätzlich Häufigkeitsangaben, ggf. Altersund Schichtangaben, Angaben zu den gemeinsam an einer Stelle vorkommenden weiteren Arten sowie in vielen Fällen am Standort gemessene (und nicht nur aus globalen Kartenwerken abgeleitete) Umweltparameter aufweisen (Dengler et al. 2011b [4]). Das ermöglichst es, ökologisch relevante Fragen etwa nach den Ursachen von Biodiversitätsmustern im kleinskaligen Bereich oder danach, ob die Arten in Pflanzengesellschaften bezüglich ihrer Merkmale und/oder ihrer phylogenetischen Verwandtschaft mehr oder weniger streuen als dies von einem Nullmodell zu erwarten wäre (z. B. Cavender-Bares et al. 2009), anhand von umfangreichen, große geografische Gebiete abdeckenden Datensätzen zu analysieren. Die retrospektive Analyse der in vielen Regionen seit 50 oder gar 100 Jahren in weitgehend einheitlicher Weise erhobenen Vegetationsaufnahmen erlaubt eindeutige Quantifizierungen, wie sich Klimawandel, Nährstoffeintrag oder Landnutzungswandel auf die Verbreitung und Häufigkeit von Arten ausgewirkt hat (z. B. Lenoir et al. 2008, Dupré et al. 2010, Jandt et al. 2011). Dies ist von kaum zu unterschätzendem Wert, wenn es darum geht, die weit verbreiteten, aber recht spekulativen Zukunftsprojektionen von Biodiversität mittels species distribution modelling (z. B. Thomas et al. 2004, Pompe et al. 2008) zu ,kalibrieren“.

Ein häufiges Problem, das beim Zusammenführen und/oder gemeinsamen Auswerten von unterschiedlichen Vegetationsdatenbanken (oder ganz allgemein sippenbezogenen biologischen Datenbanken, wie etwa Kartierungsdaten, Sammlungsdaten, Merkmalsdaten, Gefährdungsdaten) 
auftritt, sind unterschiedliche taxonomische Auffassungen (Jansen \& Dengler 2008 [6], 2010 [7]). Vielfach wird dieses Problem unterschätzt, da die Datenbankmanager vermuten, dass es sich einfach darum handelt, Synonyme, also verschiedene Namen für den gleichen Inhalt, aufeinander ,abzubilden“. Dies wäre datenbanktechnisch noch relativ einfach und weitgehend automatisch lösbar, wobei auch hier gerade in Regionen mit langer floristischer Tradition die Vielzahl unterschiedlicher Florenwerke in oftmals zahlreichen Auflagen, von denen fast nie zwei die gleiche taxonomische Sicht vertreten, einen erheblichen Aufwand verursachen kann. Das ungleich größere, den meisten mit floristisch-vegetationskundlichen Datenbanken Befassten aber nicht bewusste Problem stellt die - taxonomisch völlig korrekte - Anwendung eines Namens in unterschiedlich weit gefasstem Sinne dar (Berendsohn 1995, Koperski et al. 2000; siehe Abb. 6), welches sich nur eingeschränkt automatisiert lösen lässt (Jansen \& Dengler 2010 [7]). In Jansen \& Dengler (2010) [7] erörtern wir die mit Pflanzennamen in Vegetationsdatenbanken verknüpften Probleme und stellen in Jansen \& Dengler (2008) [6] mit der GermanSL eine taxonomische Referenzliste vor, welche die Flora von Deutschland (Gefäßpflanzen, Moose, Flechten, Makroalgen) in einer für Vegetationsdatenbanken adäquaten Weise elektronisch abbildet.

\begin{tabular}{|c|c|c|c|c|}
\hline \multirow[t]{3}{*}{ Ascherson (1864) } & \multicolumn{4}{|c|}{ F. ovina L. } \\
\hline & \multicolumn{3}{|c|}{ F. ovina ssp. vulgaris Koch } & $\begin{array}{l}\text { F. ovina ssp. } \\
\text { duriuscula (L.) }\end{array}$ \\
\hline & F. ovina ssp. v & jaris var. vulgaris & \begin{tabular}{|c|} 
F. ovina ssp. \\
vulgaris var. \\
tenuifolia (Sibth.) \\
Ascherson
\end{tabular} & \\
\hline \multirow[t]{3}{*}{ Jäger \& Werner (2005) } & \multicolumn{4}{|c|}{ F. ovina agg. } \\
\hline & \multicolumn{2}{|c|}{ F. ovina L. } & \begin{tabular}{|c|}
$\begin{array}{c}\text { F. filiformis } \\
\text { Pourr. }\end{array}$ \\
\end{tabular} & $\begin{array}{c}\text { F. brevipila } \\
\text { Tracey }\end{array}$ \\
\hline & $\begin{array}{c}\text { F. ovina ssp. } \\
\text { ovina }\end{array}$ & $\begin{array}{c}\text { F. ovina ssp. } \\
\text { guestfalica } \\
\text { (Boenn. ex } \\
\text { Rchb.) K. Richt. }\end{array}$ & & \\
\hline \multirow[t]{2}{*}{ Wisskirchen \& Haeupler (1998) } & \multicolumn{4}{|c|}{ F. ovina agg. } \\
\hline & F. ovina L. & $\begin{array}{l}\text { F. guestfalica } \\
\text { Boenn. ex Rchb. }\end{array}$ & $\begin{array}{l}\text { F. filiformis } \\
\text { Pourr. }\end{array}$ & $\begin{array}{l}\text { F. brevipila } \\
\text { Tracey }\end{array}$ \\
\hline
\end{tabular}

Abb. 6: Drei unterschiedliche Gliederungskonzepte der Gattung Festuca (aus Jansen \& Dengler 2010 [7]). Senkrecht untereinander stehende Elemente bezeichnen inhaltliche Entsprechungen. Der Artname $F$. ovina L. ist jeweils taxonomisch korrekt angewandt und meint doch drei verschiedene Inhalte (Taxonyme oder taxon views). Ohne die taxonomische Sichtweise des Bearbeiters zu kennen, ist daher keine inhaltlich korrekte Zuordnung eines Datenbankeintrags ,Festuca ovina L.“ möglich. 


\section{Theorie, Simulationen und Analysemethoden zur Skalenabhän- gigkeit von Biodiversität [8-13]}

\subsection{Artenzahl-Areal-Beziehungen: Analysemethoden und generelles Konzept}

Als fundamentalster und offensichtlichster Aspekt der Skalenabhängigkeit von Biodiversität beschäftigen Artenzahl-Areal-Beziehungen (species-area relationships, SARs) die Ökologen schon lange und intensiv (z. B. Arrhenius 1920, Gleason 1922, Preston 1962, Connor \& McCoy 1979, 2001, Williamson 1988, Rosenzweig 1995, Lomolino 2001, Drakare et al. 2006, Guilhaumon et al. 2008). Ein wesentlicher Aspekt dieser Analysen ist das Bemühen, diejenige mathematische Funktion zu finden, welche die Beziehung zwischen Flächengröße $(A)$ und Artenzahl $(S)$ am besten beschreibt, da dies einerseits das theoretische Verständnis befördert, andererseits die praktische Anwendung, etwa bei der Extra- oder Interpolation von Artenreichtumsdaten, erleichtern würde. Anfangs wurden in der Diskussion in der Regel nur die Potenzfunktion (power function; Arrhenius 1920, Preston 1962) und die Logarithmusfunktion (logarithm function, oftmals fälschlich als Exponentialfunktion bezeichnet; Gleason 1922) betrachtet. Dies lag vor allem daran, dass diese Funktionstypen durch eine Logarithmus-Transformation einer oder beider Achsen einer einfachen Analyse mittels linearer Regression zugänglich waren, wie auch eine lineare Beziehung zwischen Artenzahl und Flächengröße, die aber allgemein als ungeeignet für diesen $\mathrm{Zu}$ sammenhang angesehen wird (Connor \& McCoy 1979). In jüngerer Zeit wurden zahlreiche weitere Funktionstypen für die Modellierung von Artenzahl-Areal-Beziehungen vorgeschlagen (Reviews in Tjørve 2003, 2009, Dengler 2009a [8]).

Studien zur Eignung unterschiedlicher Funktionstypen weisen bis in die jüngste Zeit oft fundamentale methodische Probleme auf, die ihre Ergebnisse zweifelhaft erscheinen lassen. Diese wurden von mir erstmals umfassend zusammengestellt (Dengler 2008 [9], 2009a [8]):

1. In der Literatur ist die Typologie von Artenreichtums-Beziehungen (species richness relationships) trotz verschiedener terminologischer Vorschläge (Gotelli \& Colwell 2001, Scheiner 2003, 2004, Gray et al. 2004a, 2004b, Scheiner et al. 2011) weiterhin inkonsistent und widersprüchlich. Deswegen habe ich eine neue Typologie vorgeschlagen, die die Unvollständigkeiten und Widersprüchlichkeiten bisheriger Ansätze zu überwinden versucht (Dengler 2009a [8]). Besonders strittig sind hier Artenreichtums-Beziehungen, deren einzelne Datenpunkte jeweils einer Gesamtfläche entsprechen, die aus vielen nicht zusammenhängenden Einzelflächen besteht. Obwohl hier Artenreichtum flächenbezogen ermittelt wird, sollte man solche Kurven nicht als Artenzahl-Areal-Beziehung bezeichnen, sondern zu den Artenzahl- 
Erhebungsaufwands-Beziehungen (species-sampling relationships oder species-sampling effort relationships; Dengler 2009a [8], Azovsky 2011) stellen, mit denen sie grundlegende mathematische Eigenschaften teilen. Autoren, die meinen, hier handle es sich um eine normale Artenzahl-Areal-Beziehung, kommen oftmals zu unberechtigten Schlussfolgerungen (z. B. Stiles \& Scheiner 2007).

2. Einige vorgeschlagene Funktionstypen sind theoretisch unmöglich: So sagt die oft verwendete Logarithmusfunktion mathematisch zwingend negative Artenzahlen auf den kleinsten Flächengrößen voraus. Viele Funktionen beinhalten auch einen Sättigungswert, d. h. einen oberen Grenzwert der Artenzahl, der asymptotisch erreicht wird, was für ArtenzahlErhebungsaufwands-Beziehungen ein angemessenes Modell ist, nicht jedoch für ArtenzahlAreal-Beziehungen im eigentlichen Sinne (Williamson et al. 2001, 2002, ausführliche Diskussion in Dengler 2009a [8]).

3. Wenn zwecks Anwendung einer einfachen linearen Regression bestimmte Funktionstypen nicht direkt modelliert werden, sondern indirekt durch Transformation der Ordinate, man also beispielsweise $\log S$ als abhängige Größe hat, sind die Gütemaße der Anpassung nicht mit einer anderen Regression vergleichbar, in der $S$ die abhängige Größe ist (Loehle 1990, Fattorini 2007). Dieses Problem betrifft viele Vergleiche zwischen Logarithmus- und Potenzfunktion in der Literatur, da letztere oftmals in der Form $\log S=\log c+z \log A$ anstelle von $S=c A^{z}$ modelliert wurde.

4. Wenn Modelle mit einer unterschiedlichen Anzahl von Funktionsparametern verglichen werden, muss der unterschiedlichen Modellkomplexität durch ein geeignetes statistisches Gütemaß Rechnung getragen werden (Quinn \& Keough 2002, Johnson \& Omland 2004). Das vielfach verwendete $R^{2}$ ist dann ungeeignet und sollte am besten durch AICc (Akaike Information Critierion corrected for small $n$ ) ersetzt werden (Burnham \& Andersson 2002).

5. Die Beurteilung der Eignung einer bestimmten Funktion kann alternativ für $S$ oder für $\log S$ erfolgen, es gibt a priori keinen Grund, die eine oder die andere Lösung zu bevorzugen (Williamson 1988, Quinn \& Keough 2002). Als drittes Beurteilungskriterium habe ich den log error of extrapolation (LEE) eingeführt, der die Extrapolationsgüte von Funktionen beurteilt, indem die (bekannten) Artenzahlen auf der größten Fläche mit denjenigen verglichen werden, die von einer Regression des gleichen Funktionstyps vorhergesagt werden, die nur auf kleineren Flächengrößen basiert (Dengler 2009a [8]).

Ausgehend von diesen fünf Überlegungen habe ich anhand von 14 nested-plot-Datensätzen in kontinuierlicher Vegetation und von 6 Inseldatensätze jeweils 12 mögliche Funktionstypen so- 
wohl im $S$-Raum als auch im $\log S$-Raum verglichen (Dengler 2009a [8]). Ein typisches Ergebnis ist für fünf Funktionstypen in Abb. 7 visualisiert.
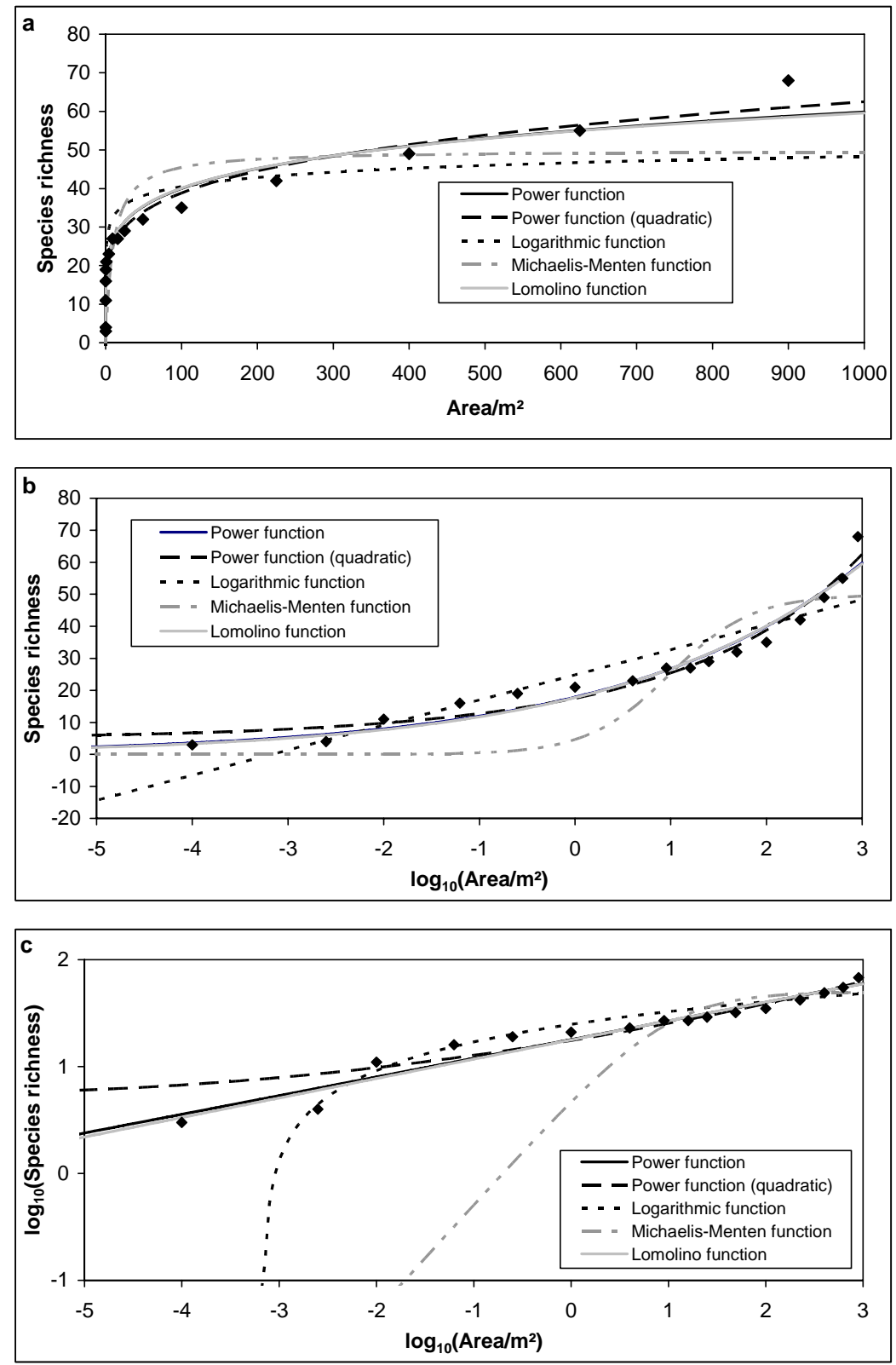

Abb. 7: Beispiel für die Anpassung von fünf verschiedenen Funktionstypen an Artenzahl-Areal-Daten einer Trockenrasengesellschaft, dargestellt in drei arithmetischen Räumen (aus Dengler 2008 [9]). Potenzfunktion (power function) und Logarithmusfunktion (logarithmic function) sind unbegrenzt wachsende Funktionen mit zwei Funktionsparametern; die quadratische Potenzfunktion (power function [quadratic]) ist eine drei-parametrige Variante der Potenzfunktion mit skalenabhängig zu- oder abnehmendem $z$-Wert; Michaelis-Menten- und Lomolino-Funktion sind „Sättigungsfunktionen“ mit zwei bzw. drei Parametern. In diesem Beispiel war die quadratische Potenzfunktion (beurteilt nach AICc im $S$-Raum) das beste Modell, mit geringem Abstand gefolgt von der normalen Potenzfunktion $(\triangle \mathrm{AICc}=0,43)$; die Eignung der Lomolino-Funktion war schon deutlich geringer $(\triangle \mathrm{AICc}=4,67)$, während die Logarithmus- und die Michaelis-Menten-Funktion, wie bereits visuell erkennbar, unangemessen für die Daten waren $(\triangle \mathrm{AICc}=21,24$ bzw. 33,01). 
Für die nested-plot-Daten erwiesen sich Potenzfunktion, quadratische Potenzfunktion, Lomolino-Funktion und die kumulative beta-P-Funktion nach der Anpassungsgüte als die geeignetsten Modelle. Von diesen war die Extrapolationsgüte (LEE) der normalen, im $S$-Raum angepassten Potenzfunktion am besten war. Bei den Insel-Daten waren die die Ergebnisse in der Grundtendenz ähnlich, doch gab es viel mehr Unterschiede zwischen den einzelnen Datensets als bei den nested-plot-Daten. Das lässt sich leicht dadurch erklären, dass im Gegensatz zu einem nestedplot-Datensatz bei einem Insel-Datensatz nicht nur die Flächengröße, sondern auch die Umweltbedingungen notwendigerweise von Datenpunkt zu Datenpunkt variieren und damit mehr „Rauschen“" in die Daten kommt (Dengler 2009a [8]).

Während in der Betrachtung der Kurvenanpassung und Extrapolationsgüte die LomolinoFunktion und die kumulative beta-P-Funktion sich kaum von der Potenzfunktion und deren quadratischer Variante unterschieden, rate ich in der Gesamtabwägung von der Anwendung der beiden erstgenannten Funktionen ab (Dengler 2009a [8]). Gegen sie spricht (a) der Umstand, dass sie eine theoretisch nicht begründbare Artensättigung auf großen Flächen unterstellen, (b) dass ihre Parameter schlecht zu interpretieren und (c) die Parameterschätzungen sehr instabil sind. Die quadratische Potenzfunktion eignet sich zwar in kleinen Skalenabschnitten hervorragend um eine graduelle Änderung des $z$-Wertes zu „simulieren“, doch sagt sie einen theoretisch nicht möglichen Extremwert vorher (meist allerdings außerhalb des betrachteten Kurvenbereichs). Damit bleibt die normale Potenzfunktion als bester genereller Ansatz für die Beschreibung von Artenzahl-Areal-Beziehungen übrig, der zudem den Vorteil hat, zwei gut interpretierbare Parameter $(c, z)$ zu liefern, für die es in der Literatur eine umfangreiche Vergleichsbasis gibt (z. B. Hobohm 1998, Dolnik 2003, Fridley et al. 2005, Drakare et al. 2006, Kreft et al. 2008, Schmiedel et al. 2010 [23]). Wenn man also nicht sehr viele unterschiedliche Modelle simultan vergleichen will, wie es Guilhaumon et al. (2008) oder Dengler (2009a) [8] getan haben, erscheint daher die standardmäßige Verwendung der Potenzfunktion mittels nicht-linearer Regression angepasst im $S$-Raum als bester Ansatz, ggf. gekoppelt mit einem statistischen Test, ob sich der $z$-Wert skalenabhängig ändert (vgl. two-slope model in Dengler 2010 [12]).

\subsection{Effekt von Erfassungsmethoden auf die Ergebnisse}

In Dengler (2008) [9] gebe ich einen Überblick darüber, welche - bislang oftmals ignorierten methodischen Aspekte bei kleinskaligen Artenzahl-Areal-Analysen potenziell die Ergebnisse verfälschen. Dabei stelle ich - abgesehen von den in Abschnitt 3.1 schon eingehend besprochenen adäquaten Anwendung von Regressionsverfahren und Methoden zur Beurteilung der Anpassungsgüte - vier weitere Punkte heraus: 
1. Any-part- oder grid-point-System: Eine Pflanzenart kann in einer Aufnahmefläche entweder als vorhanden gewertet werden, wenn sie in diese mit irgendeinem oberirdischen Teil hineinragt (any-part oder shoot presence), oder wenn ihr Zentroid innerhalb der Fläche liegt (gridpoint). Oftmals werden auch nur die Pflanzen gezählt, die in einer Fläche wurzeln (rooted presence), was mathematisch dem grid-point-System ähnelt. Alle Verfahren bewirken auf kleinen Flächen mathematisch unvermeidliche Abweichungen vom „,normalen“ Verlauf einer Artenzahl-Areal-Beziehung. Beim any-part-System nähert sich der $z$-Wert auf kleinen Flächen 0, beim grid-point-System dagegen 1 (Dengler 2003, Williamson 2003). Die Abweichungen beim grid-point-System fallen allerdings sehr viel stärker aus und treten schon bei relativ größeren Flächen auf (Williamson 2003; siehe auch Dengler 2005 [15]).

2. Form der Aufnahmeflächen: Langestreckte Flächen beinhalten im Mittel mehr Arten als kompakte Flächen der gleichen Größe (Kunin 1997, Bossuyt \& Hermy 2004, Stohlgren 2007).

3. Räumlicher Zusammenhang der Aufnahmeflächen: Es ist eine allgemein anerkannte ökologische Tatsache, dass eine aus nicht zusammenhängenden Teilen zusammengesetzte „Fläche“ aufgrund (a) des distance decay in den abiotischen Standortbedingungen (Nekola \& White 1999) und (b) der starken Distanzabhängigkeit biologischer Prozesse (wie etwa Ausbreitung) im Mittel mehr Arten beinhalten wird als eine zusammenhängende Fläche gleicher Größe. Diese Tatsache macht auch den fundamentalen Unterschied zwischen SARs und SSRs aus (siehe Dengler \& Boch 2008b [11], Dengler 2009a [8]).

4. Replizierung und räumliche Anordnung der Aufnahmeflächen: Wenn es das Ziel ist, allein den Effekt der Fläche auf die Artenzahl zu ermitteln, sollten alle übrigen Umweltbedingungen zwischen den verschiedenen Flächengrößen konstant gehalten werden. Das bedeutet, dass die kleineren Flächengrößen eines nested-plot-Designs repliziert und ihre Artenzahlen gemittelt werden sollten. Dies gilt insbesondere für die kleinsten Flächen, auf denen Artenzahlen relativ gesehen am stärksten variieren (siehe auch Dengler 2006 [17])

Punkt 3 haben wir in Dengler \& Oldeland (2010) [10] mittels einer Simulationsstudie analysiert. In Dengler (2009a) [8] habe ich theoretisch begründet, dass Artenzahl-Areal-Beziehungen (species-area relationships, SARs) und Artenzahl-Erfassungsaufwands-Beziehungen (speciessampling effort relationships, SSRs) fundamental andere Kurvenverläufe haben, und auf dieser Basis dafür plädiert, dass Erfassungsschemata, die mathematisch die Eigenschaften von SSRs haben, auch als solche bezeichnet werden sollten, selbst wenn die Probenahme auf definierten (aber nicht zusammenhängenden!) Flächen erfolgte. Scheiner (2009; siehe auch Scheiner et al. 2011) hat gegen diese theoretische Aussage und die darauf basierende typologische Konsequenz 
vehement protestiert. Diesen Disput haben wir in Dengler \& Oldeland (2010) [10] zum Anlass genommen, um in einer Simulationsstudie zu testen, ob die von Dengler (2009a) [8] gemachten Vorhersagen zutreffen. Dazu haben wir mehrere Replikate einer virtuellen Landschaft mit $64 \times$ 64 Zellen simuliert, in der 100 Arten mit realistischen Häufigkeitsverteilungen und räumlichen Verteilungsmustern auftreten. Auf diese Landschaft haben wir dann die SAR- und die SSRErfassungsmethode (siehe Abb. 8) sowohl für die Gesamtfläche wie auch für den zentralen $32 \times$ 32 Zellen großen Ausschnitt angewandt. Das Ergebnis (Abb. 9) zeigt, dass sich die Kurvenverläufe von SARs und SSRs erheblich unterscheiden, wie von Dengler (2009a) [8] und Chiarucci et al. (2009) vorhergesagt. Im Multi-Modell-Vergleich wurden die SARs am besten durch die Potenzfunktion bzw. deren quadratische Variante, die SSRs dagegen am besten durch die Sättigungsfunktionen (Lomolino, Michaelis-Menten) abgebildet.

a)

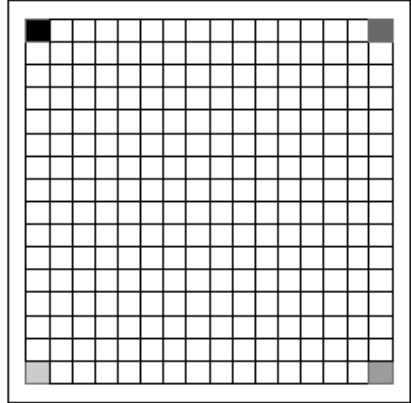

c)

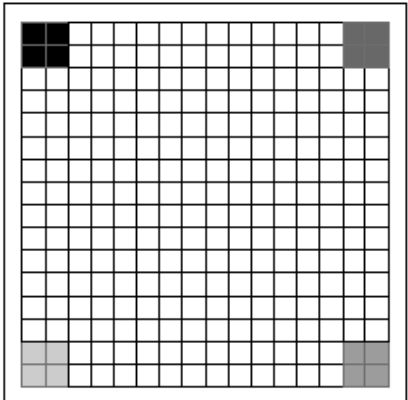

e)

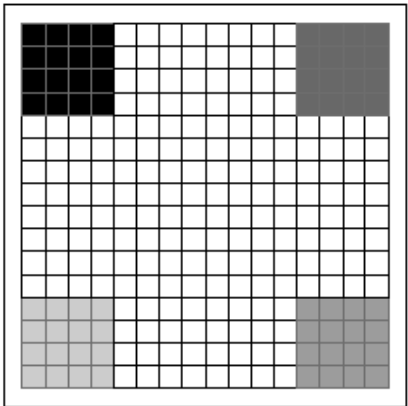

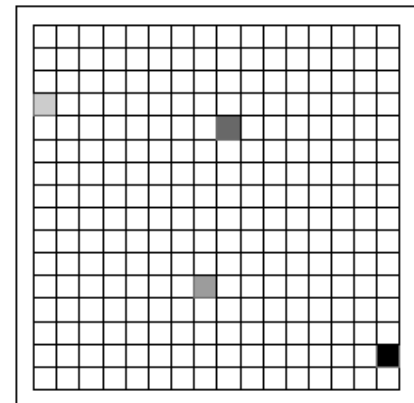

d)

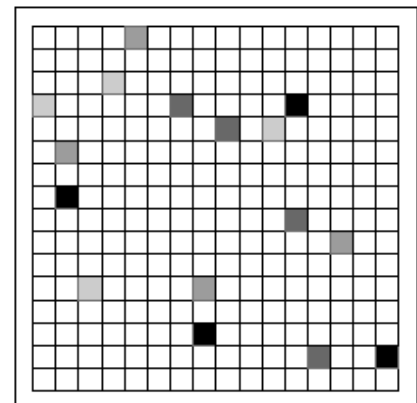

f)

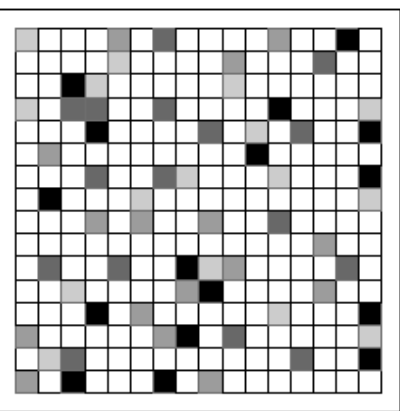

Abb. 8: Vergleich der SAR- und der SSR-Erfassungsmethode (aus Dengler \& Oldeland 2010 [10]). Die Probefläche (sampling unit) ist immer eine Rasterzelle groß, die betrachtete Korngröße (grain) steigt von oben nach unten von 1 über 4 zu 16. In allen Fällen markieren gleiche Grautöne diejenigen Rasterzellen, die zusammen als eine Einheit betrachtet werden und deren gemeinsamer Artenreichtum ermittelt wird. Der entscheidende Unterschied ist, dass bei der SAR-Methode, die Flächen zusammenhängend sind, bei der SSR-Methode dagegen nicht. 


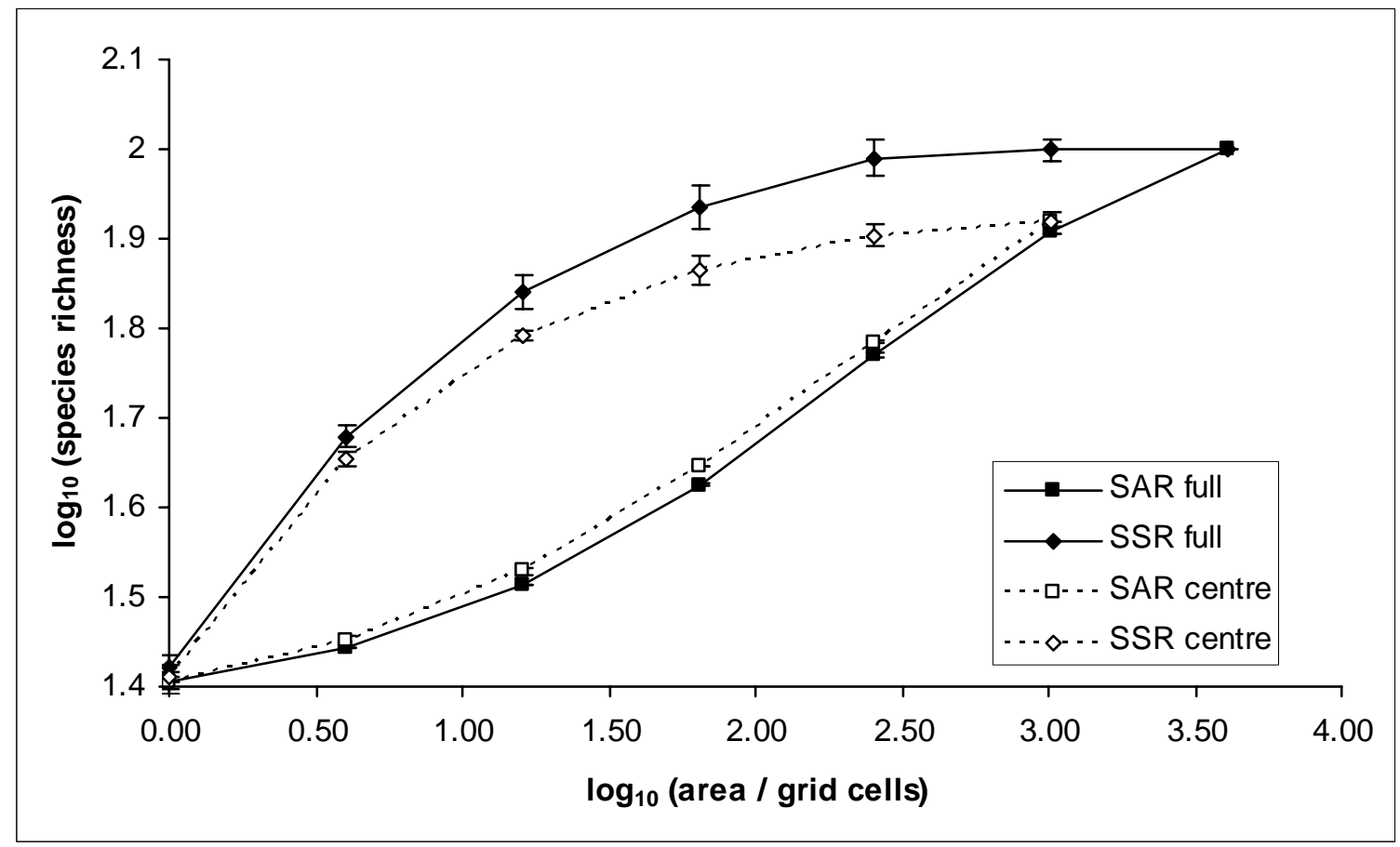

Abb. 9: Effekt der SAR- und SSR-Erfassungsmethode auf die Kurvenverläufe von Artenreichtums-Beziehungen (aus Dengler \& Oldeland 2010 [10]). Dargestellt sind die Mittelwerte ( \pm Standardfehler) aus fünf zufälligen virtuellen Landschaften mit jeweils 100 Arten $\left(\log _{10} S=2\right)$. Beprobt wurden sowohl die Gesamtfläche (full) als auch deren zentrales Viertel (centre).

Punkt 4 haben wir in Dengler \& Boch (2008b) [11] mit einer Fallstudie analysiert. Dazu haben wir anhand von realen Daten aus estnischen Trockenrasengesellschaften getestet, welchen Einfluss es auf die Modellauswahl hat, wenn man nicht mit einem nested-plot-Design und replizierten kleineren Flächengrößen arbeitet, wie von Dengler (2008) [9] und Dengler (2009a) [8] empfohlen. Dazu wurden anhand der gleichen Datensets jeweils nested-plot-SARs und SARs aus zufälligen, nicht geschachtelten Aufnahmeflächen, und Mittelwert-SARs und Einzelwert-SARs verglichen. Zwar erwies sich in allen Varianten die normale Potenzfunktion als das mit Abstand beste Modell, doch wurde die relative Eignung (ausgedrückt als $\triangle \mathrm{AICc}$ ) der ungeeigneten Logarithmus- und Michaelis-Menten-Funktion besser, wenn man statt Mittelwerten Einzelwerte oder statt nested plots random plots verwendete. Das ist genau der von Dengler (2008) [9] vorhergesagte Effekt.

\subsection{Small island effect}

Im Jahr 1963 wurde von Niering erstmals ein ,anomales“ Verhalten von Artenzahlen in Beziehung zu Flächengröße für die kleinsten Inseln eines Atolls berichtet. Die Darstellung der von ihm gefundenen Artenzahl-Areal-Beziehung im halblogarithmischen Raum suggerierte, dass 
unterhalb eines bestimmten Schwellenwertes $T$ die Artenzahl unabhängig von der Fläche variiert und erst darüber eine „normale“ Artenzahl-Areal-Beziehung auftritt. In der Folge gab es immer wieder einzelne Berichte von ähnlichen Phänomenen (Review in Dengler 2010 [12]). Lomolino \& Weiser (2001) berichten, dass dieser sogenannte small island effect (SIE) in den meisten der von ihnen analysierten Inseldatenset vorkomme, und entsprechend avancierte dieses Phänomen mehr und mehr zum ,gesicherten“ Lehrbuchwissen der Biogeografie und Makroökologie (Lomolino 2000, Lomolino et al. 2006, Whittaker \& Fernández-Palacios 2007). Doch wurden auch frühzeitig erhebliche Zweifel an den Methoden und an der Existenz des SIE überhaupt geäußert (Williamson et al. 2001, Burns et al. 2009, Dengler 2009a [8], Tjørve \& Tjørve 2011).

Dies habe ich in Dengler (2010) [12] zum Anlass genommen, alle bisher für den SIE verwendeten Definitionen und Methoden einem kritischen Review zu unterziehen, einen Vorschlag für einen methodisch einwandfreien Test auf das Vorliegen eines SIEs zu machen und diesen an einem Datenset auszuprobieren, für das kurz zuvor das Vorliegen eines SIEs behauptet worden war (Sfenthourakis \& Triantis 2009: Asselarten [Isopoda] auf Inseln der Ägäis). Es zeigte sich, dass alle bisherigen Studien, die vom Vorliegen eines SIEs berichtet haben, einen oder mehrere methodische Probleme aufweisen, von denen jedes für sich genommen die Gefahr eines falschpositiven Befundes erhöhen (Dengler 2010 [12]: p. 258). Das häufigste Problem ist wie bei normalen SAR-Analysen (siehe Dengler 2009a [8]), dass $R^{2}$ als Gütemaß verwendet wurde, wenn Funktionen unterschiedlicher Komplexität verglichen wurden. Wenn man eine Potenzfunktion und ihre SIE (breakpoint-low) Variante anhand von $R^{2}$ vergleicht, wird die Version mit SIE mathematisch notwendig immer gleich gut oder besser abschneiden. Statistisch korrekt hätte der zusätzliche Parameter $T$ durch die Verwendung von AICc als Gütemaß „,bestraft“ werden müssen. Ein besonders eklatantes Problem ist die Verwendung der Logarithmusfunktion für Inseln mit Artenzahlen, die nur geringfügig über 0 liegen. Hier wird, da eine Logarithmusfunktion immer (unmögliche) negative Artenzahl für kleine Flächen vorhersagt (siehe Abb. 10), die Modellauswahl aus mathematischer Notwendigkeit der SIE-Variante der Logarithmusfunktion den Vorzug vor ihrer normalen Variante geben, was dann nur Folge der generellen Nicht-Eignung der Logarithmusfunktion ist, und kein Beleg für einen SIE. Einen ähnlichen Effekt hätte das vielfach zu beobachtende, statistisch aber nicht statthafte Weglassen von Inseln ohne Arten aus den Analysen (Williams 1996). Weitere methodische Probleme sind in Dengler (2010) [12] ausführlich dargestellt. 


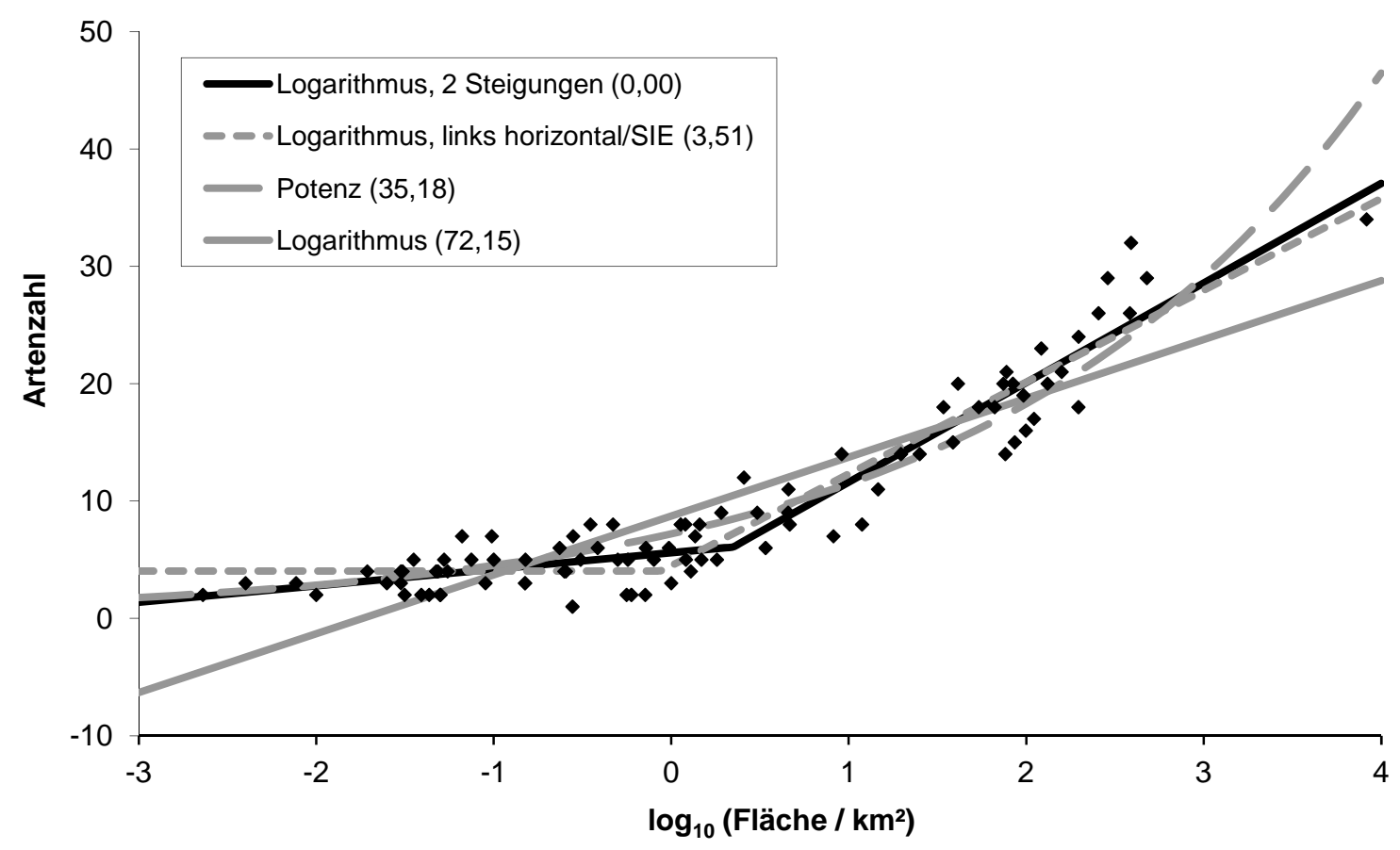

Abb. 10: Artenreichtum von Asseln (Isopoda) auf 90 Inseln unterschiedlicher Größe in der Ägäis (modifiziert aus Dengler 2010 [12]). Es sind vier verschiedene Funktionen vergleichend dargestellt, wobei die Zahlen in Klammern deren $\triangle \mathrm{AICc}-$ Wert darstellen ( 0 bezeichnet das beste Modell).

Die Re-Analyse der Daten von Sfenthourakis \& Triantis (2009) mit statistisch korrekten Verfahren und unter Berücksichtung von weiteren möglichen Funktionstypen zeigte, dass in diesem Fall tatsächlich kein SIE gemäß den Definitionen von MacArthur \& Wilson (1967), Lomolino (2000) und Triantis et al. (2006) vorlag. In diesem Fall erwies sich das two-slope-Modell, das unter einem gewissen Schwellenwert eine andere (geringere), aber nicht fehlende Steigung der Artenzahl-Areal-Kurve vorhersagt, als das beste Modell (Abb. 10). Inzwischen wurden sämtliche Datensets von Lomolino \& Weiser (2001) in einer von mir betreuten Bachelor-Arbeit mit den Methoden aus Dengler (2010) [12] re-analysiert (Wallenfang 2011). Es zeigte sich, dass meine Vermutungen aufgrund der methodischen Probleme in dieser und anderen Studien alle zutrafen. Fälle, bei denen die Artenzahl-Areal-Kurve im linken Teil statistisch abgesichert tatsächlich nicht ansteigt, waren sehr viel seltener, als von Lomolino \& Weiser (2001) behauptet. Insbesondere traten Fälle ohne Steigung im rechten Kurvenabschnitt fast genauso häufig auf (Wallenfang 2011), was impliziert, dass dieses Muster nichts mit der Größe der Inseln zu tun hat, sondern Ausdruck eines „stochastischen Rauschens“ ist, das bei Insel-SARs im Gegensatz zu nested-plotSARs statistisch unvermeidlich ist (vgl. Dengler \& Boch 2008b [11]). 


\subsection{Extrapolation von Artenzahlen}

Die Extrapolation von Artenzahlen auf größere Flächen, die einer direkten Erhebung nicht oder nur schwerlich zugänglich sind, ist eine der wesentlichen Anwendungen von ArtenreichtumsBeziehungen (species richness relationships, SRRs im Sinne von Scheiner et al. 2011) ganz allgemein (z. B. Colwell \& Coddington 1994, Gotelli \& Colwell 2001, Dengler 2009a [8], Scheiner et al. 2011). Es gibt vier wesentliche methodische Ansätze zur Extrapolation: (a) ArtenzahlAreal-Beziehungen (SARs); (b) Artenzahl-Erhebungsaufwands-Beziehungen (SSRs); (c) sogenannte non-parametric richness estimators (Magurran 2004); (d) ein jüngst von Harte et al. (2009) an hochrangiger Stelle vorgeschlagenes, anderweitig aber noch nicht getestetes Verfahren, das eine Hochrechnung mit Hilfe von mittlerer Artendichte und mittlerer Individuendichte auf einer beliebigen kleineren Skala ermöglichen soll.

Angesichts der großen Bedeutung der Artenreichtums-Extrapolation ist es erstaunlich, dass praktisch noch nie das Verhalten dieser vier unterschiedlichen Ansätze im gleichen System miteinander verglichen und zu einer bekannten Gesamtartenzahl in Relation gesetzt wurde. Die wenigen systematischen Vergleiche, die bislang publiziert wurden, fanden normalerweise nur innerhalb einer der vier Kategorien und oftmals in Unkenntnis der tatsächlichen Gesamtartenzahl eines Systems statt, etwa nur zwischen den verschiedenen non-parametric richness estimators (Review in Magurran 2004). Die Studie von Dengler \& Oldeland (2010) [10] betritt insofern wichtiges methodisches Neuland, indem sie zumindest zwei der methodischen Ansätze in einem System (virtuelle Landschaft) mit bekannter Gesamtartenzahl vergleicht. Es zeigte sich, dass bei korrekter Anwendung sowohl SARs als auch SSRs in der Lage sind, Artenzahlen auf einer vierfach größeren Fläche recht genau vorherzusagen. Es müssen allerdings zwei entscheidende methodische Aspekte beachtet werden, ohne die eine korrekte Vorhersage nicht möglich ist (Dengler \& Oldeland 2010 [10]):

1. Es müssen geeignete Funktionstypen für die Extrapolation verwendet werden, d. h. Potenzfunktion (ggf. auch Lomolino-Funktion) für SARs und eine Sättigungsfunktion (z. B. Michaelis-Menten oder Lomolino) für SSRs.

2. Bei SSRs kann immer nur genau auf jene Fläche (extent) extrapoliert werden, für die die Erhebung gedacht war (d. h. innerhalb deren Grenzen die einzelnen sampling units zufällig verteilt waren), während bei SARs mit nur unwesentlichen Fehlern auch auf größere (oder kleinere) Flächen als den extent extrapoliert werden kann.

Während diese Simulationsstudie nach meinem Wissen die erste Arbeit war, in der zwei der vier fundamentalen Extrapolationsansätze miteinander und mit einer bekannten Gesamtartenzahl verglichen wurden, hat inzwischen eine von mir initiierte und betreute Diplomarbeit anhand von 
realen Felddaten alle vier Ansätze verglichen und kommt zu qualitativ ähnlichen Ergebnissen (Peters 2010).

\subsection{Auswirkungen der Skalenabhängigkeit von Biodiversität auf Anwendun- gen}

Während ich in den bisherigen vier Abschnitten von Kapitel 3 den Einfluss der Fläche auf die Artenzahl behandelt habe, soll es in diesem letzten Abschnitt um den Einfluss der Fläche auf die sogenannte Stetigkeit (constancy) gehen. Anders als die diversen Artenreichtums-Beziehungen fand dieser Aspekt der Skalenabhängigkeit bislang so gut wie keine Beachtung in der Literatur (siehe aber Du Rietz 1922, Moravec 1973, Nosek 1986, Dengler 2003, Šizling \& Storch 2004). Dabei steht die Stetigkeits-Areal-Beziehung in einem engen Zusammenhang mit der ArtenzahlAreal-Beziehung, da letztere einfach das Integral der Stetigkeits-Areal-Beziehungen über alle Arten darstellt (Storch et al. 2008, Dengler et al. 2009 [13]).

Die Stetigkeits-Areal-Beziehung hat nicht nur theoretische Bedeutung im Kontext der verschiedenen skalenabhängigen makroökologischen Muster (Šizling \& Storch 2004, Storch et al. 2008), sondern auch eminent praktische Bedeutung, da Stetigkeit eine vielfach angewandte Größe ist und die vorherrschende Unkenntnis über ihrer Skalenabhängigkeit leicht zu Fehlschlüssen führt. So dürfen etwa die Stetigkeiten von erwünschten (z. B. Rote Liste) oder unerwünschten Arten (z. B. Neophyten) nicht zwischen Vegetationstypen oder Behandlungsvarianten verglichen werden, wie dies in der Literatur oft geschieht, wenn nicht die Verwendung einheitlicher Flächengrößen sichergestellt ist. Die größte praktische Relevanz hat die Stetigkeit aber in der Vegetationsklassifikation, da sowohl „klassische“ (Braun-Blanquet 1964, Bergmeier et al. 1990, Dengler 2003, Tsiripidis et al. 2009, Willner et al. 2009) also auch „statistische“ Verfahren (z. B. Hill 1979, Bruelheide 2000, Chytrý et al. 2002) zu Bestimmung des diagnostischen Wertes von Arten implizit nahezu immer die Stetigkeiten von Arten in verschiedenen Aufnahmenkollektiven vergleichen. Die traditionelle, aber falsche Annahme in der Pflanzensoziologie war, dass Flächengröße keine Rolle mehr spiele, sobald man sich jenseits eines bestimmten „MinimumAreals“ befindet (Braun-Blanquet 1964, Westhoff \& van der Maarel 1973, Barkman 1989, Dierschke 1994). Obwohl das Konzept des Minimum-Areals unvereinbar mit den seit Langem bekannten fundamentalen Erkenntnissen zu Artenzahl-Areal-Beziehungen ist (siehe Abschnitte 1.5 und 3.1), findet man es weiterhin auch in biologischen und ökologischen Lehrbüchern, die nichts mit der pflanzensoziologischen Tradition zu tun haben (z. B. Sitte et al. 2002: p. 987, Tremp 2005). 
Eine Analyse realer Daten zeigt jedoch, dass mit zunehmender Flächengröße nicht nur weitere Arten hinzukommen, sondern die Stetigkeit der zuvor schon vorhandenen Arten auch zunimmt (Abb. 11b). Letztlich macht mit zunehmender Flächengröße jede Art einen „Übergang“ von 0 \% Stetigkeit zu 100 \% Stetigkeit durch; häufige und seltene Arten unterscheiden sich nur darin, bei welcher Flächengröße ihre 50 \%-Schwelle liegt. Dieser Übergang lässt sich mathematisch wie folgt beschreiben (Dengler et al. 2009 [13]):

$$
C(A)=1-\left(1-C_{0}\right)^{\left(\frac{A}{A_{0}}\right)^{d},}
$$

wobei $C$ die Stetigkeit und $A$ die Fläche, $A_{0} / C_{0}$ das Wertepaar für eine bekannte Flächengröße und $d$ ein empirisch zu ermittelnder „Dämpfungskoeffizient“ sind.
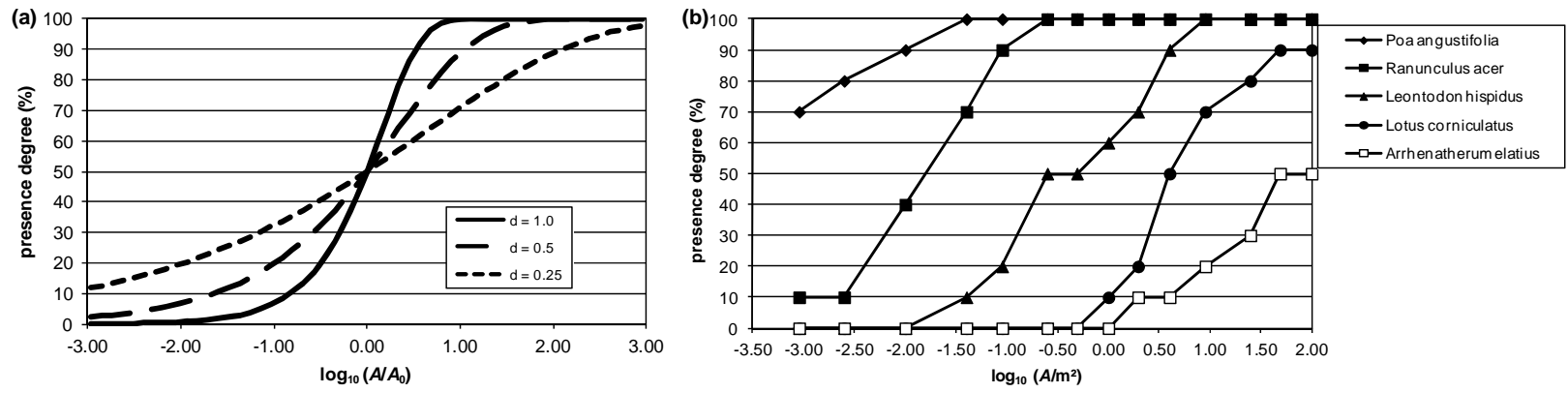

Abb. 11: Abhängigkeit der Stetigkeit von Arten von der betrachteten Aufnahmeflächengröße (modifiziert aus Dengler et al. 2009 [13]). Teilabbildung (a) zeigt theoretische Funktionen für $C_{0}=0,5$ und $d=1,0,5$ und 0,25. Teilabbildung (b) zeigt empirische „Stetigkeitsübergänge“ im Sanguisorbo-Deschampsietum (nach Daten von Moravec 1973).

In Dengler et al. (2009) [13] konnten wir anhand umfangreicher realer nested-plot-Daten aus unterschiedlichen Vegetationstypen zeigen, dass die theoretisch vorhergesagten StetigkeitsAreal-Beziehungen immer in dieser Form auftreten, nur die $d$-Werte gewissen fallspezifischen Einflüssen unterworfen sind. Die Bestimmung der $d$-Werte erlaubte es uns, (a) zu ermitteln, wie stark Stetigkeitswerte in Folge unterschiedlicher Flächengrößen verzerrt sein können und (b) eine ungefähre Umrechnungsformel auf eine Standardflächengröße vorzuschlagen. Es zeigte sich, dass Flächengrößen einen stark verzerrenden Einfluss auf die Ergebnisse haben, wenn sie um mehr als den Faktor 5 variieren. Durch die große und noch nicht genau verstandene Variabilität von $d$, ist die Hoffnung auf eine zuverlässige Umrechnung von Stetigkeitswerten auf andere Flächengrößen trügerisch und es scheint für die Zukunft weitaus sinnvoller, Erhebungen mit wenigen standardisierten Flächengrößen durchzuführen (Chytrý \& Otypková 2003, Dengler 2003; siehe auch Abschnitt 2.1). 


\section{Artendiversität auf verschiedenen Skalenebenen [14-25]}

\subsection{Artenzahl-Areal-Beziehungen (Europa)}

Um zu ergründen, welchen Gesetzmäßigkeiten Artenzahl-Areal-Beziehungen in verschiedenen Pflanzengesellschaften im kleinskaligen Bereich folgen, haben wir unterschiedlichste Trockenrasentypen der estnischen Insel Saaremaa analysiert (Dengler \& Boch 2008b [11]). Dafür lagen sorgfältig erhobene Artenzahlen für Gefäßpflanzen, Moose und Flechten (incl. epiphytischer und epilithischer Sippen) für Flächengrößen von $1 \mathrm{~cm}^{2}$ bis $100 \mathrm{~m}^{2}$ vor, wobei alle Flächengrößen unter $100 \mathrm{~m}^{2}$ gemäß den Vorschlägen von Dengler (2008) [9], 2009b [1]) fünffach repliziert waren und als Mittelwerte Eingang in die Analysen fanden. Wir haben fünf Funktionstypen, die sich hinsichtlich ihres generellen Verlaufes (mit/ohne Aysmptote) und der Anzahl der Parameter (2 bzw. 3) unterscheiden (Tab. 2; siehe auch Abb. 7), in einem auf AICc basierenden Multimodellvergleich (Burnham \& Anderson 2002) analysiert. Es zeigte sich, dass in 69 \% der Fälle ein einfaches Potenzgesetz und in 31 \% der Fälle ein Potenzgesetz mit quadratischem Term die Artenzahlzunahme mit der Fläche am besten beschreibt. Im $S$-Raum war in keinem der analysierten Bestände eine andere Funktion jemals die beste und die hohen $\triangle \mathrm{AICc}-$ Werte (Lomolino: 7,6; Logarithmus: 30,3; Michaelis-Menten: 30,6) zeigen, dass sie keinen Erklärungswert hatten (Dengler \& Boch 2008b [11]). Die im $S$-Raum gefittete normale Potenzfunktion war auch hinsichtlich der Extrapolationsgüte am überzeugendsten mit einem LEE-Wert von $-0,02 \pm 0,03$, was anzeigt, dass im Mittel eine leichte Unterschätzung stattfand. Bei den quadratischen Potenzfunktionen war das quadratische Glied immer sehr gering und teils positiv, teils negativ.

Tab. 2: Die fünf in der Arbeit von Dengler \& Boch (2008b) [11] verglichenen Funktionstypen zur Beschreibung von Artenzahl-Areal-Beziehungen. $S=$ Artenreichtum; $A=$ Fläche; $b_{i}=$ modellierter Parameter. Bei der Potenzfunktion werden $b_{0}$ and $b_{1}$ meist als $c$ und $z$ bezeichnet. Es ist zu beachten, dass im Falle der quadratischen Potenzfunktion die ermittelten Extremwerte of weit außerhalb der betrachteten Flächengrößen liegen, der Effekt des quadratischen Gliedes also auf einen allmählichen Anstieg oder Abstieg der relativen Steigung beschränkt.

\begin{tabular}{llllc}
\hline Funktionsname & Model & Kurvenverlauf & $\begin{array}{l}\text { Anzahl } \\
\text { Parameter }\end{array}$ & $\begin{array}{c}\text { Obere As- } \\
\text { ymptote }\end{array}$ \\
\hline Potenz (normal) & $S=b_{0} A^{b 1}$ & Unbegrenzt, konvex & 2 & nein \\
Potenz (quadratisch) & $S=10^{\wedge}\left(b_{0}+b_{1} \log A+b_{2}(\log A)^{2}\right)$ & $\begin{array}{l}\text { u-förmig }\left(b_{2}>0\right) \text { oder } \\
\text { umgkehrt u-förmig }\left(b_{2}<0\right)\end{array}$ & 3 & nein \\
Logarithmus & $S=b_{0}+b_{1} \log A$ & Unbegrenzt, konvex & 2 & nein \\
Michaelis-Menten & $S=b_{0} A /\left(b_{1}+A\right)$ & Sättigung, konvex & 2 & $\mathrm{ja}\left(b_{0}\right)$ \\
Lomolino & $S=b_{0} /\left(1+\left(b_{1} \log (b 2 / A)\right)\right)$ & Sättigung, sigmoid & 3 & $\mathrm{ja}\left(b_{0}\right)$ \\
\hline
\end{tabular}


Die Befunde von Dengler \& Boch (2008b) [11] unterstützen den Vorschlag von mir (Dengler 2009a [8]), die Potenzfunktion als einfachen und gut begründeten Standardansatz zur Analyse von Artenzahl-Areal-Beziehungen auf jedweder räumlichen Skalenebene zu verwenden und ggf. auf die Veränderung der $z$-Werte in Abhängigkeit von der räumlichen Skala zu testen, was geschehen kann, indem man (a) eine quadratische Potenzfunktion bzw. (b) ein two-slope-Modell in den Modellvergleich mit einbezieht (vgl. Dengler 2010 [12]) oder (c) die $z$-Werte abschnittsweise berechnet und dann mittels Varianzanalyse (ANOVA) vergleicht. Das extrem schlechte Abschneiden der Logarithmusfunktion im Vergleich mit der Potenzfunktion, wie ich dies auch bei einer Analyse von nested-plot-Daten unterschiedlichster Vegetationstypen in Dengler (2009a) [8] gefunden habe, widerlegt empirisch, die von manchen Vegetationskundlern angenommene besondere Eignung dieses Funktionstyps im kleinskaligen Bereich (Gleason 1922, Stohlgren et al. 1995, He \& Legendre 1996, van der Maarel 1997). Eine solche Überlegenheit der Logarithmusfunktion gerade in diesem Bereich kann wegen ihrer fundamentalen Probleme mit der korrekten Beschreibung von Artenzahlen nahe 0 (vgl. Dengler 2010 [12], siehe Abschnitt 3.3) auch theoretisch nicht gegeben sein kann.

Wenn man mit der Potenzfunktion als Grundmodell arbeitet, eine eventuelle Skalenabhängigkeit aber in Betracht zieht, kann man vielfach zu aufschlussreichen Ergebnissen kommen, von denen ich hier drei exemplarisch benennen möchte:

1. Löbel et al. (2004) [14] haben auf Öland Trockenrasengesellschaften, die ökologische jenen auf Saaremaa (Dengler \& Boch 2008b [11]) gleichen, mit ähnlichen Methoden analysiert. Der einzige wesentliche Unterschied war, dass Löbel et al. (2004) [14] mit rooted presence $(\approx$ grid point-System) gearbeitet haben, Dengler \& Boch (2008b) [11] dagegen mit shoot presence (= any part-System) (vgl. Williamson 2003, Dengler 2008 [9]), siehe Abschnitt 3.2). Der Effekt dieses Methodenunterschiedes war erheblich: Anders als Dengler \& Boch (2008b) [11] fanden Löbel et al. (2004 [14]; für Details siehe Löbel 2002) durchgängig quadratische Potenzfunktionen als beste Modelle, wenn alle Flächengrößen ab $1 \mathrm{~cm}^{2}$ berücksichtigt wurden. Unabhängig davon, welche Gesellschaft oder welche der Artengruppen (alle oder Gefäßpflanzen, Moose und Flechten getrennt) betrachtet wurde, waren die Kurven im doppellogarithmischen Raum immer deutlich konvex (Löbel et al. 2004 [14], was einem erheblichen quadratischen Glied von durchschnittlich -0,06 entspricht (Löbel 2002). Wenn nur der Bereich ab $100 \mathrm{~cm}^{2}$ angeschaut wurde, verschwand die Kurvatur (in log-log-Darstellung) weitgehend und die Artenzahl-Areal-Beziehung ließ sich hervorragend durch eine normale Potenzfunktion beschreiben. Damit liegt erstmals ein unabhängiger Beleg für das von Williamson (2003) theoretisch vorhergesagte und an einem Beispiel aus dem tropischen Regenwald 
belegte „Aufsteilen“ von SARs zu kleinen Flächen hin vor, wenn mit dem grid-point-System gearbeitet wird. Dieser Befund unterstützt den in Abschnitt 3.2 gemachten Vorschlag, vorzugsweise mit dem any-part-System zu arbeiten.

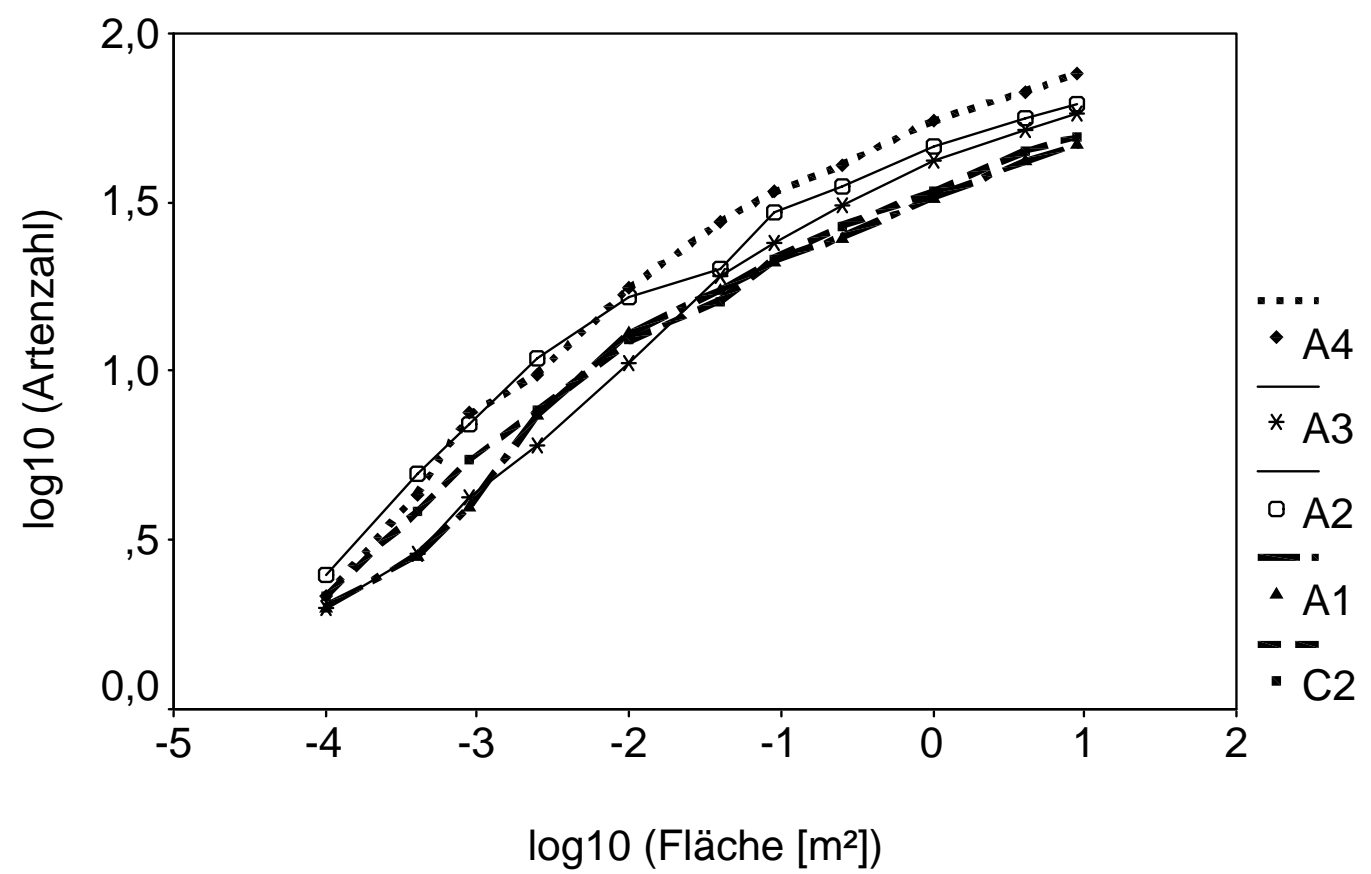

Abb. 12: Gemittelte Artenzahl-Areal-Kurven für Trockenrasengesellschaften des Großen Alvars auf der schwedischen Ostseeinsel Öland, erhoben mit dem grid-point-System (aus Löbel et al. 2004 [14]). A1 = Crepido pumilaeAllietum alvarensis; A2 = Fulgensio bracteatae-Poetum alpinae; A3 = Helianthemo oelandici-Galietum oelandici; A4 = Gypsophilo fastigiatae-Globurlarietum vulgaris; C2 = Veronico spicatae-Avenetum. Zu den Vegetatationseinheiten vgl. Löbel \& Dengler (2008) [32] und Abschnitt 5.2).

2. In uckermärkischen Trockenrasen haben wir in einem Studentenkurs Flächen bis hinunter zu $1 \mathrm{~mm}^{2}$ mit dem any-part-System analysiert (Dengler 2005 [15]). Dazu hatten wir ein Gestell, an dessen oberen Ende präzise Bohrungen angebracht waren, durch die „Stempel“ mit definierter Fläche langsam abgesenkt werden konnten. Dabei wurde von der Seite beobachtet, ob und welche Pflanzenteile getroffen wurden. Erstaunlicherweise ließen sich die ArtenzahlAreal-Beziehungen hier meist bis hinunter zu den Kleinstflächen sehr gut mit einem normalen Potenzgesetz beschreiben. Nur in wenigen Fällen (Beispiel siehe Abb. 13) trat ein merkliches Abflachen am linken Rand der Kurve auf, wie von Dengler (2003) und Williamson (2003) vorhergesagt. Das zeigt, dass die im any-part-System zu Kleinstflächen hin auftretenden Irregularitäten so minimal sind und erst bei so winzigen Flächen auftreten, dass sie für praktische 
Anwendungen i. d. R. bedeutungslos sein dürften, anders als die Abweichungen beim gridpoint-System (s. o.).

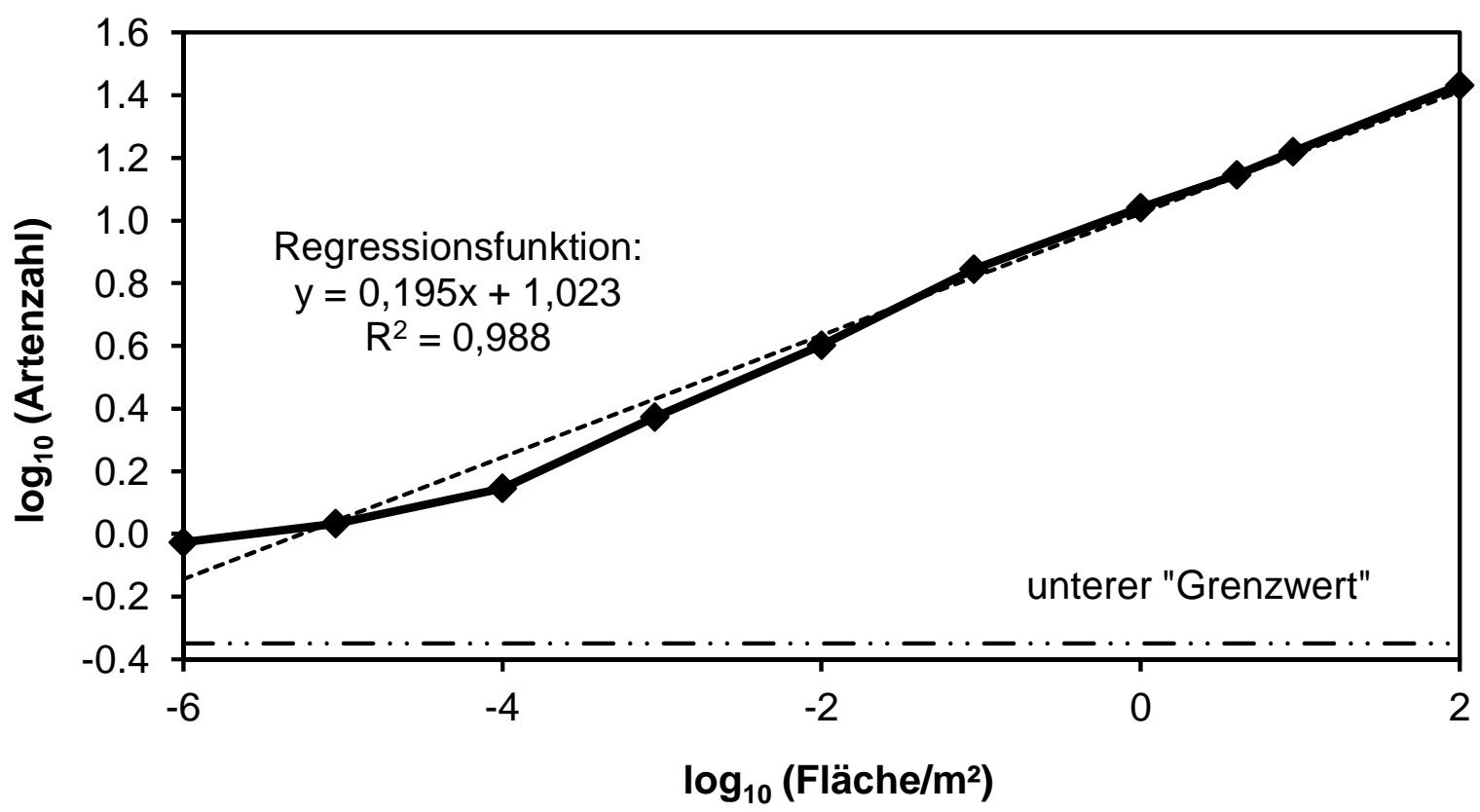

Abb. 13: Beispiel einer Artenzahl-Areal-Kurve für einen Bestand des Corniculario-Corynephoretum in der Uckermark (NO-Brandenburg), erhoben mit dem any-part-System (verändert aus Dengler 2005 [15]). Die Kurve zeigt unterhalb von $1 \mathrm{~cm}^{2}$ ein deutliches Abflachen. Der geschätzte untere Grenzwert, d. h. der Logarithmus der Deckung $(45 \%)=-0,35$, wenn man unterstellt, dass sich die Arten kaum überdecken, ist ebenfalls dargestellt.

3. Bei einer Analyse von Artenzahl-Areal-Beziehungen an Aufnahmeflächen, die im Bereich des Messtischblattes Lüneburg (TK 2628) zufallsverteilt und ohne Rücksicht auf Homogenitätskriterien eingerichtet wurden (Allers \& Dengler 2007 [16]), zeigte sich, dass die z-Werte von den kleinsten zu den größeren Flächen deutlich anstiegen (Abb. 14). Crawley \& Harral (2001) haben bei systematischen Erhebungen in Silwood Park ein ganz ähnliches Muster gefunden, für das bislang keine überzeugende Erklärung vorliegt. Möglicherweise hat sich in diesem Fall dadurch, dass in Form von großen Stauden, Sträuchern und Bäumen viel größere Organismen Berücksichtigung fanden als in den Trockenrasen von Beispiel 2 der Punkt des Abflachens der Artenzahl-Areal-Kurve deutlich weiter nach rechts verschoben. Hier besteht weiterer Forschungsbedarf. 


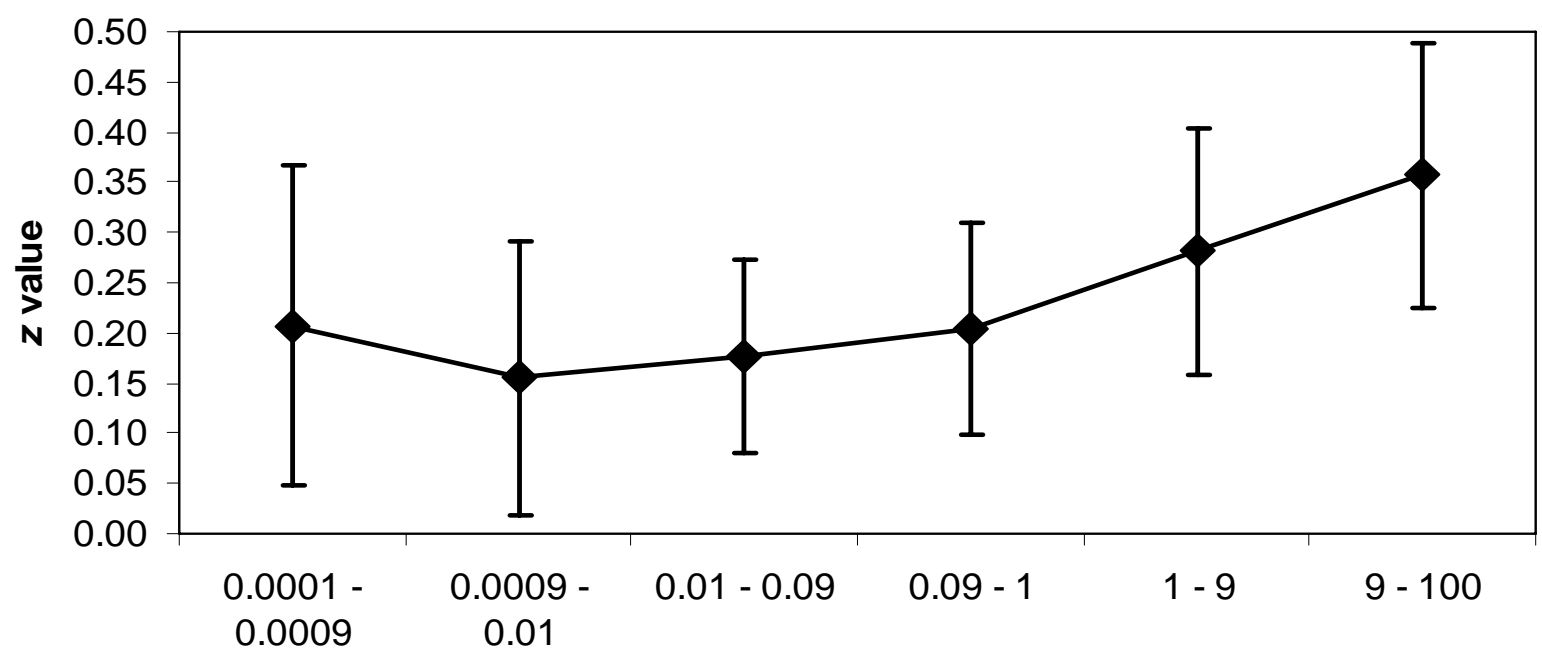

Plot size range $\left[\mathrm{m}^{2}\right]$

Abb. 14: Skalenabhängige Variabilität $\operatorname{der} z$-Werte in zufallsverteilten nested-plot-Serien auf dem Messtischblatt Lüneburg (TK 2728) (verändert aus Allers \& Dengler 2007 [16]). Auf der Abszisse sind die jeweiligen Flächengrößenübergänge, auf der Ordinate die „lokalen“ $z$-Werte aufgetragen. Dargestellt sind Mittelwerte und Standardabweichungen aus insgesamt 50 solchen Serien.

Die in den verschiedenen Untersuchungen ermittelten $z$-Werte liegen alle im Bereich 0,18-0,28 für Trockenrasen (Dengler 2005 [15], Dengler \& Boch 2008b [11]) bzw. 0,15-0,41 für zufällig in der mitteleuropäischen Landschaft verteilte Aufnahmeflächen (Allers \& Dengler 2007 [16]), mit einem Mittelwert von in beiden Fällen 0,22. Damit liegen die Werte alle in einem recht engen Bereich und nahe an 0,25, was generell als typischer $z$-Wert gilt (Williamson 1988, Gaston \& Spicer 2004). Warum z-Werte nicht viel mehr im insgesamt möglichen Bereich zwischen 0 und 1 variieren, für verschiedene Habitate und Taxa so ähnlich sind und sich zudem oft über viele Größenordnungen der Fläche kaum verändern (wenn man leicht erklärliche Artefakte wie die Auswirkungen des grid-point-Systems auf kleinen Flächen ignoriert), ist eine der großen, noch nicht zufriedenstellend geklärten Fragen der Biodiversitätsforschung. Jedenfalls deuten die von mir (und Mitarbeitern) in verschiedenen Pflanzengesellschaften erhobenen Werte (neben den zitierten Daten auch zahlreiche unpublizierte) darauf hin, dass $z$-Werte innerhalb von Vegetationstypen erheblich mehr variieren als zwischen Vegetationstypen. Da $z$-Werte ein Maß für $\beta$ Diversität sind (siehe Abschnitt 1.5), lassen sich positive oder negative Abweichungen vom Durchschnitt als besonders hoher bzw. niedriger räumlicher Arten-Turnover interpretieren.

\subsection{Weitere Skalenabhängigkeiten (Europa)}

Neben Artenzahl-Areal-Beziehungen gibt es noch weitere interessante Phänomene der Skalenabhängigkeit in Pflanzengesellschaften, wovon ich mich in Dengler (2006) [17] mit zweien be- 
schäftigt habe. Zunächst gehe ich anhand von Daten, die in uckermärkischen Trockenrasen nach dem in Dengler (2009b) [1] vorgestellten Erfassungssystem erhoben wurden, der Frage nach, wie sich die Variabilität der Artenzahl skalenabhängig (grain) ändert. Es zeigte sich hier wie auch an Datensätzen von Trockenrasen Ölands und der Kurischen Nehrung (Russland), dass die absolute Standardabweichung monoton mit der Flächengröße ansteigt, die relative Streuung (Variationskoeffizient) dagegen monoton abnimmt. Diese Gesetzmäßigkeit ließ sich in beiden Fällen sehr gut mit einer steigenden bzw. fallenden Exponentialfunktion in Bezug auf die logarithmierte Fläche beschreiben (Abb. 15).
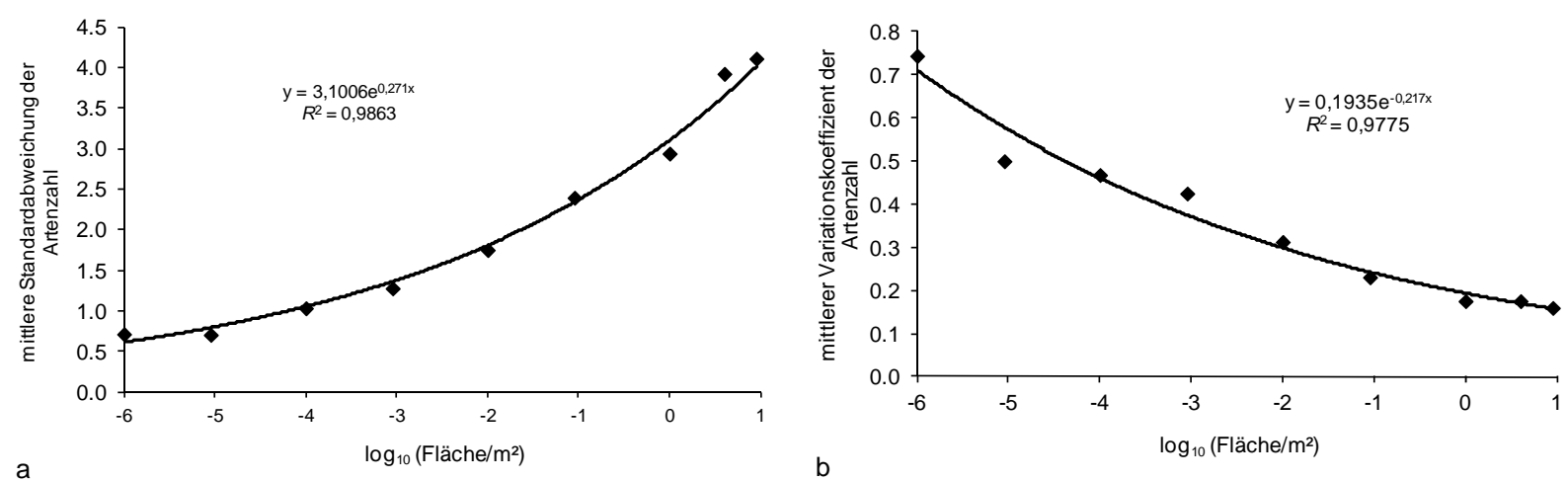

Abb. 15: Variabilität von Artenzahlen (a: Standardabweichung; b: Variationskoeffizient) auf unterschiedlich großen

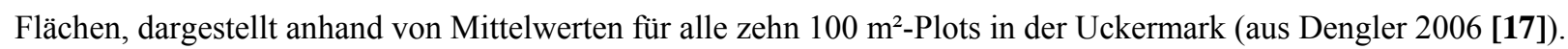

Die Skalenabhängigkeit des Variationskoeffizienten (CV) der Artenzahl $S$ in Abhängigkeit von der Fläche $A$ gehorcht also der Gleichung $C V=b_{0} \cdot \mathrm{e}^{b_{1} \cdot \log _{10}\left(A / \mathrm{m}^{2}\right)}$. In Dengler (2006) [17] habe ich die Funktionsparameter $b_{1}$ und $b_{2}$ für unterschiedliche Trockenrasentypen in drei europäischen Regionen bestimmt. Die gefundene Regelhaftigkeit lässt sich verwenden, um zu quantifizieren, wie viel stärker repliziert kleinere Aufnahmeflächengrößen sein müssen, um die dort vorliegende Artendichte mit gleicher Genauigkeit wie auf einer größeren Fläche zu ermitteln (Tab. 3). 
Tab. 3: Mindestanzahl von Replikaten, um in einem visuell homogen erscheinenden Trockenrasenbestand mittlere Artendichten auf den kleineren Flächen mit der gleichen Genauigkeit wie auf der größten untersuchten Fläche zu ermitteln. Die Angaben basieren auf den mittleren, minimalen und maximalen Exponenten $b_{1}$ (Werte in Klammern). In der letzten Zeile ist der Faktor angegeben, um den die Zahl der Replikate bei jeder Verringerung der Flächengröße auf ein Zehntel erhöht werden muss (verändert nach Dengler 2006 [17]).

\begin{tabular}{llcccc}
\hline & $\begin{array}{c}\text { Flächengröße } \\
\text { (Vielfaches der } \\
\text { Startfläche) }\end{array}$ & $\begin{array}{c}\text { Mittel } \\
(\mathbf{- 0 , 2 6 4 )}\end{array}$ & $\begin{array}{c}\text { Min. } \\
(\mathbf{- 0 , 1 7 3 )}\end{array}$ & $\begin{array}{c}\text { Max. } \\
(\mathbf{- 0 , 4 4 5 )}\end{array}$ & $\begin{array}{c}\text { Vorschlag } \\
\text { Barkman } \\
(\mathbf{1 9 8 9})\end{array}$ \\
\hline Startfläche $\left(A_{0}\right)$ & 1 & 1 & 1 & 1 & 1 \\
Kleinere Flächen & 0,1 & 2 & 1 & 2 & 2 \\
& 0,01 & 3 & 2 & 6 & 5 \\
& 0,001 & 5 & 3 & 14 & 10 \\
& 0,0001 & 8 & 4 & 35 & 20 \\
& 0,00001 & 14 & 6 & 86 & 50 \\
\hline Faktor für $A=A_{0} / 10$ & 0,000001 & 24 & 8 & 208 & 100 \\
\hline
\end{tabular}

Weiterhin betrachte ich in Dengler (2006) [17] die Frage, ob sich bestimmte Aspekte von Biodiversität in Trockenrasen entfernungsabhängig verändern, man also den für abiotische Umweltbedigungen (Nekola \& White 1999) oder großskalige Biodiversitätsmuster (Qian et al. 2005, Qian \& Ricklefs 2007) wohlbekannten distance decay auch für kleinskalige Biodiversitätsmuster belegen kann (vgl. Palmer \& White 1994). Der Nachweis einer solchen Entfernungsabhängigkeit für eine Facette der Biodiversität, würde zugleich implizieren, dass keine Homogenität vorliegt. Ich habe die Entfernungsabhängig von Unterschieden in den Artenzahlen und in der floristischen Ähnlichkeit (Sörensen-Index) für einen Trockenrasentyp der Uckermark (Festucion valesiacae; $n=15$ ) und einen der Insel Öland (Crepido-Allietum; $n=66$ ) beispielhaft analysiert. In beiden Fällen zeigte der Sörensen-Index einen deutlichen und signifikanten Entfernungsabfall, während die Artendichten sich entweder nicht signifikant oder zwar signifikant, aber in vernachlässigbarem Umfang veränderten. Die Befunde zeigen, dass auch visuell homogen erscheinende Bestände einer Vegetationseinheit nicht wirklich homogen sind, sondern eine deutliche räumliche Strukturierung ihrer Artenzusammensetzung aufweisen.

\section{$4.3 \alpha$ - und $\beta$-Diversitätsmuster (Europa)}

In Turtureanu \& Dengler (im Druck) [18] gehen wir der Frage nach, wie sich ein $\alpha$ Diversitätsmaß und fünf verschiedene $\beta$-Diversitätsmaße in Relation zu einander verhalten und wie sie von abiotischen Umweltparametern abhängen. Als Modellsystem haben wir hierfür die 
Gefäßpflanzenvegetation von Waldlichtungen unterschiedlicher Größe in den rumänischen Karpaten ausgewählt (Abb. 16). Bei der Erstellung minimaler adäquater Modelle mittels GLMMs (generalized linear mixed models), zeigte, dass die sechs verglichenen Facetten der Gefäßpflanzendiversität von unterschiedlichen Umweltfaktoren beeinflusst wurden, und hier selbst die fünf Varianten von $\beta$-Diversität trotz gleichen Datensatzes und gleicher Artengruppe ein sehr verschiedenes Verhalten zeigten (Tab. 4). Für die kleinskalige $\alpha$-Diversität waren Kronendachöffnung (positiv) und Laubstreubedeckung (negativ) die entscheidenden Faktoren. Von den $\beta$ Diversitätsmaßen wurden zwei durch die Spannweite der Kronendachöffnung (positiv), eines durch die Meereshöhe und zwei durch keinen der berücksichtigten Umweltfaktoren erklärt. Dieses Bild differenzierte sich noch weiter, wenn man statt der Artenzahl insgesamt die Grasland-, Wald- und Saumarten (vgl. Abschnitt 5.3) getrennt betrachtete (Turtureanu \& Dengler im Druck [18]). Diese Befunde zeigen, wie wichtig es einerseits für eine umfassende Biodiversitätsbetrachtung ist, unterschiedliche Biodiversitätsfacetten parallel zu analysieren, dass andererseits Aussagen zu Biodiversität ,im Allgemeinen“ mit Vorsicht zu betrachten sind.

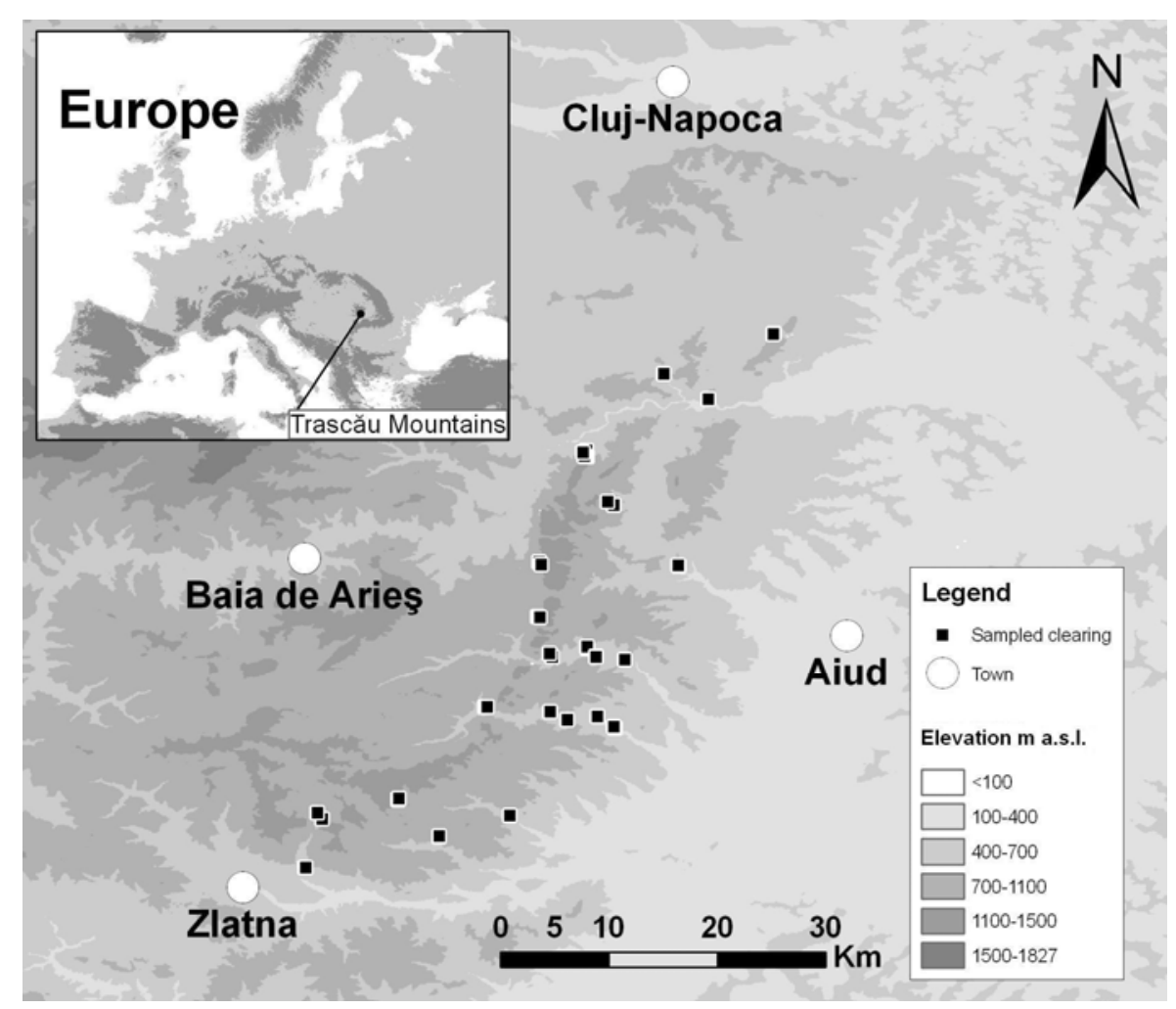

Abb. 16: Untersuchte Waldlichtungen in den Trascău-Bergen, einem Teil der rumänischen Karpaten (aus Turtureanu \& Dengler im Druck [18]). 
Tab. 4: Minimal adäquate Modelle für die Abhängigkeit von sechs verschiedenen Biodiversitätsfacetten der Gefäßpflanzenartenzahl (gesamt) von Umweltparametern, berechnet mit einem GLMM ( $\alpha$-Diversität, $n=160)$ bzw. multiplen linearen Regressionen ( $\beta$-Diversität, $n=40$ ). Die Modelle entstanden durch schrittweise Vereinfachung aus dem vollen Modell, das im Falle der $\alpha$-Diversität die Parameter Kronendachöffnung, Laubstreubedeckung, Hangneigung und heat load index sowie die Identität der Lichtung umfasste. Im Falle der $\beta$-Diversitäten fanden die Mittelwerte und Spannweiten der vier erstgenannten Umweltparameter sowie Gesteinstyp (sauer oder basisch) und Meereshöhe Berücksichtigung (verändert aus Turtureanu \& Dengler im Druck [18]).

\begin{tabular}{|c|c|c|c|c|}
\hline Diversitätsfacette & $\begin{array}{l}\text { Mittelwert } \\
\quad( \pm \text { SD) }\end{array}$ & Parameter & $\begin{array}{l}\text { Standardi- } \\
\text { sierter Koef- } \\
\text { fizient }\end{array}$ & $p$-Wert \\
\hline \multirow[t]{2}{*}{$\alpha$ : Artenreichtum auf $1 \mathrm{~m}^{2}$} & $17.17( \pm 4.94)$ & Kronendachöffnung & 0.146 & 0.007 \\
\hline & & Laubstreubedeckung & -0.118 & 0.002 \\
\hline$\beta_{\text {Add }}:$ Additive $\beta$-Diversität & $20.03( \pm 4.88)$ & $\begin{array}{l}\text { Kronendachöffnung: } \\
\text { Spannweite }\end{array}$ & 0.452 & 0.003 \\
\hline$\beta_{\text {Mult: }}$ Multiplikative $\beta$-Diversität & $2.17( \pm 0.21)$ & Meereshöhe & 0.330 & 0.037 \\
\hline $\begin{array}{l}\beta_{\text {distance decay:Steigung der distance-decay- }} \\
\text { Beziehung }\end{array}$ & $0.02( \pm 0.01)$ & - & & \\
\hline $\begin{array}{l}\beta_{\text {SOR }} \text { : Sørensen-basierte multivariate } \beta \text { - } \\
\text { Diversität }\end{array}$ & $0.64( \pm 0.05)$ & $\begin{array}{l}\text { Kronendachöffnung: } \\
\text { Spannweite }\end{array}$ & 0.322 & 0.043 \\
\hline $\begin{array}{l}\beta_{\text {SIM }} \text { : Simpson-basierte multivariate } \beta \text { - } \\
\text { Diversität }\end{array}$ & $0.57( \pm 0.07)$ & - & & \\
\hline
\end{tabular}

\subsection{Abhängigkeit der Artendiversität von der Umwelt (Europa)}

Anhand des umfangreichen Datensatzes der von mir betreuten Diplomarbeit von Löbel (2002) konnten wir in Löbel et al. (2006) [19] umfassend der Frage nachgehen, welche Faktoren den Pflanzenartenreichtum im kleinskaligen Bereich (grain $=4 \mathrm{~m}^{2}$ ) bestimmen. Dafür standen uns 452 Vegetationsaufnahmen von sämtlichen Trockenrasentypen im Südteil der schwedischen Insel Öland zur Verfügung (Abb. 19). Die große Besonderheit dieses Datensatzes ist, dass Löbel (2002) nicht nur die Gefäßpflanzen, sondern auch die Moose und Flechten umfassend bearbeitet und uns zudem in jeder Aufnahmefläche zahlreiche Umweltparameter erhoben hat. Während die meisten anderen Publikationen zu Diversitätsmustern in der Vegetation nur eines dieser drei Taxa betrachten, konnten wir alle drei vergleichend analysieren und zudem mögliche Interaktionen zwischen diesen hinterfragen. 


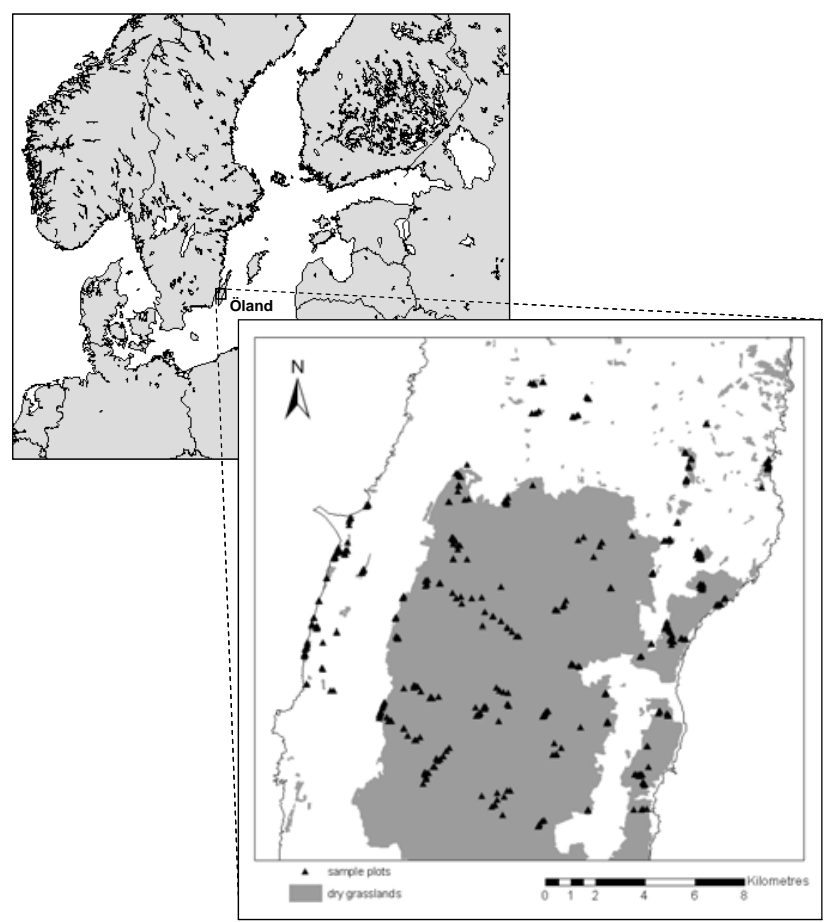

Abb. 17: Untersuchungsgebiet im Süden der schwedischen Insel Öland mit Verbreitung der Trockenrasen (grau) und Lage der Vegetationsaufnahmeflächen (schwarz) (aus Löbel et al. 2006 [19]).

Für jede der 452 Vegetationsaufnahmen standen uns Artenzahlen und Deckung gesamt sowie für jedes der drei Großtaxa sowie die folgenden Prediktoren zur Verfügung: Vegetationsdeckung gesamt und pro Großtaxon, Anteil offener Felsen (\%), Bodentiefe (cm), Mikrotopografie (cm), Landnutzung (beweidet, gemäht, ungenutzt), pH-Wert des Oberbodens, Größe des jeweiligen Trockenrasengebietes und Anteil von Trockenrasen in einem Umkreis mit Radius $r$ um die Aufnahmefläche (mit $r=100 \mathrm{~m}, 250 \mathrm{~m}, 500 \mathrm{~m}, 1 \mathrm{~km}$ und 2,5 km). Mit diesen Daten haben wir zunächst Generalized Linear Mixed Models (GLMMs) mit allen Umweltparametern mit Ausnahme jener zur Raumstruktur gerechnet. Im Ausgangsmodell wurden dabei die einfachen und quadrierten Variablen sowie deren Interaktionsterme berücksichtigt. In einer schrittweisen Modellvereinfachung wurde die Zahl der Prediktoren so lange reduziert, bis nur noch signifikante zurückblieben. Anschließend haben wir getestet, ob die Hinzunahme von Raumparametern (Größe des Trockenrasengebietes und Isolation) die Modelle noch weiter verbessern. Abschließend haben wir die Effekte der Deckungen der einzelnen Artengruppen einmal auf die Vielfalt der Artengruppen, zum anderen auf deren Residuen aus den zuvor berechneten GLMMs betrachtet.

Die Artenvielfalt ingesamt und in den Großtaxa wurde am stärksten vom pH-Wert des Bodens beeinflusst, wobei der Effekt immer positiv war, mit einer deutlichen quadratischen Komponente (entsprechend einem unimodalen Verlauf) bei den Gefäßpflanzen (Abb. 18, Tab. 5). Dies entspricht dem bekannten Muster, dass Artendiversität von Pflanzen in gemäßigten und 
borealen Breiten mit zunehmendem pH-Wert meist ansteigt, was oftmals damit erklärt wird, dass in den glazialen Refugien etwa der mittel- und nordeuropäischen Flora überwiegend basische Gesteine vorherrschten (Pärtel 2002, Ewald 2003b, Schuster \& Diekmann 2003). Ein etwas überraschender Befund ist die u-förmige Beziehung von Gesamt-, Moos- und Flechtenartenzahlen zur Bodentiefe, d. h. hier wurden die niedrigsten Artenzahlen bei mittleren Tiefgründigkeiten gefunden. Offener Felsen erwies sich als förderlich für die Vielfalt von Flechten, aber nachteilig für jene der Gefäßpflanzen. Erhöhte Mikrotopografie (verursacht etwa durch Frostwechselereignisse oder Viehtritt) hatte einen positiven Einfluss auf die Artendichte von allen Gruppen außer den Flechten und unterstützt somit die Theorie, dass kleinräumige abiotische Heterogenität wesentlich zur Aufrechterhaltung einer hohen Biodiversität beiträgt (Grubb 1977, Palmer 1994, Tilman 1994). Der Landschaftskontext hatte ebenfalls einen Einfluss auf die kleinräumige Diversität, indem sich die Größe des Trockenrasengebietes und der Anteil von Trockenrasen in der Umgebung meist positiv auswirkten. Unerwarteterweise war die Nutzung in keinem der Modelle ein relevanter Aspekt.

Bei der Analyse möglicher Interaktionen zwischen den Taxa schien es bei alleiniger Betrachtung der Artenzahlen zunächst so, als hätte die Gefäßpflanzendeckung einen starken negativen Effekt auf die Vielfalt in den beiden Nicht-Gefäßpflanzengruppen (Abb. 19). Dieses Muster verschwand aber, wenn man statt dessen die GLMM-Residuen betrachtete (Abb. 19), was zeigt, dass die Gefäßpflanzen hier wohl nicht ursächlich sind, sondern eine Kollinearität mit anderen Umweltfaktoren vorliegt.
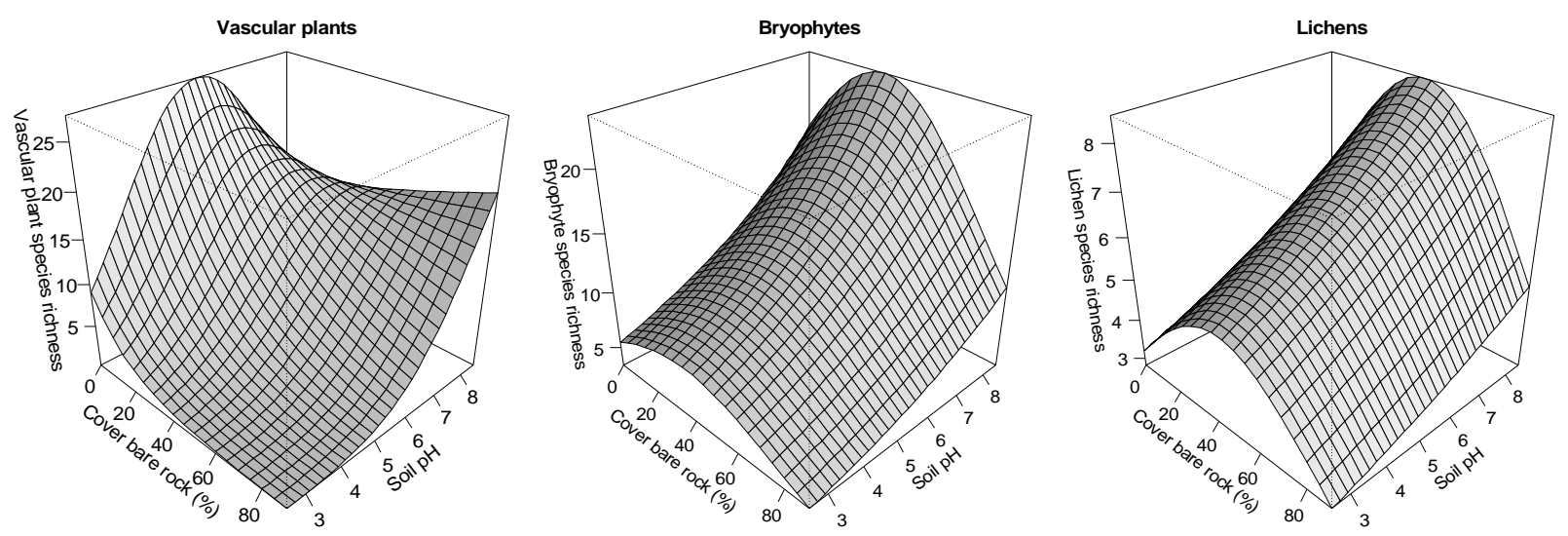

Abb. 18: Effekte der des Boden-pH-Wertes und des Anteils an der Obefläche anstehender Felsen auf die Artenvielfalt von Gefäßpflanzen, Moosen und Flechten in öländischen Trockenrasen auf $4 \mathrm{~m}^{2}$ (verändert aus Löbel et al. 2006 [19]). Dargestellt sind die mittels GLMM modellierten response surfaces (anhand der partiellen Regressionskoeffizienten). 
Tab. 5: GLMMs für die Abhängigkeit der Pflanzenartenzahlen (4 $\left.\mathrm{m}^{2}\right)$ von Umweltparametern in öländischen Trockenrasen (verändert aus Löbel et al. 2006 [19]). Alle signifikanten Parameter $(p<0,05)$ sind dargestellt unter Angabe der Richtung der Beziehung. Grau hinterlegte Zellen stehen für höchstsignifikante Zusammenhänge $(p<$ $0,001)$.

\begin{tabular}{|c|c|c|c|c|}
\hline & Alle Arten & Gefäßpflanzen & Moose & Flechten \\
\hline Mittlere Artendichte auf $4 \mathrm{~m}^{2}$ & 40,7 & 23,6 & 11,4 & 5,4 \\
\hline Räumliche Autokorrelation bis & $160 \mathrm{~m}$ & $600 \mathrm{~m}$ & $160 \mathrm{~m}$ & $300 \mathrm{~m}$ \\
\hline Boden-pH & + & + & + & + \\
\hline$(\text { Boden-pH })^{2}$ & n. s. & - & n. s. & n. s. \\
\hline Bodentiefe & - & n. s. & - & - \\
\hline$(\text { Bodentiefe })^{2}$ & + & n. s. & + & + \\
\hline Anteil offener Felsen & + & - & n. s. & + \\
\hline$(\text { Anteil offener Felsen) })^{2}$ & - & n. s. & - & - \\
\hline Mikrotopografie & + & + & + & n. s. \\
\hline$(\text { Mikrotopografie })^{2}$ & n. s. & n. s. & n. s. & + \\
\hline Boden-pH $\times$ Anteil offener Felsen & n. s. & + & n. s. & n. s. \\
\hline Boden-pH $\times$ Mikrotopografie & - & + & n. s. & n. s. \\
\hline Fläche des Trockenrasengebietes & + & n. s. & + & + \\
\hline Anteil von Trockenrasen innerhalb von $500 \mathrm{~m}$ & + & n. s. & n. s. & + \\
\hline Fläche $\times$ Anteil von Trockenrasen & - & n. s. & n. s. & n. s. \\
\hline
\end{tabular}
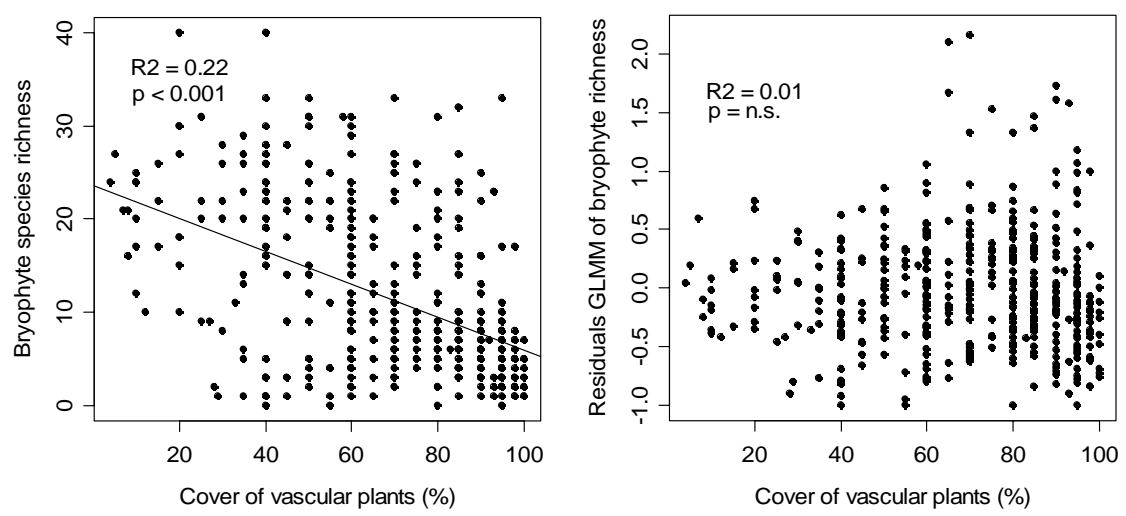

Abb. 19: Abhängigkeit von (a) der Moosartenzahl auf $4 \mathrm{~m}^{2}$ und (b) der Residuen des GLMMs für die Moosartenzahl (in Abhängigkeit von den Umweltvariablen) von der Deckung der Krautschicht in Trockenrasen der Insel Öland (verändert aus Löbel et al. 2006 [19]). 


\subsection{Abhängigkeit der Artendiversität von der menschlichen Nutzung (Euro-} pa)

Zwar gehört der Mensch auch zur Umwelt anderer Organismen; da die menschliche Nutzung aber einen „besonderen“ Umweltfaktor darstellt, habe die drei europäischen Beiträge zu diesem Themenkomplex in einen eigenen Abschnitt zusammengestellt. Zwei beschäftigen sich mit der Auswirkung unterschiedlicher menschlicher Nutzungen auf die Gefäßpflanzendiversität in Trockenrasen (Ruprecht et al. 2009 [20], Vassilev et al. 2011 [21]), der dritte dagegen mit jener auf die Diversität epiphytischer Nicht-Gefäßpflanzen in Wäldern (Friedel et al. 2006 [12]).

Das Siebenbürgische Becken in Rumänien beherbergt derzeit noch besonders großflächige und artenreiche Trockenrasengesellschaften, doch sind diese auch dort durch Änderungen der Landnutzung bedroht, insbesondere Aufgabe der vormals vorherrschenden Schaf- oder Rinderbeweidung sowie Aufforstung mit der nicht-heimischen Pinus nigra. In Ruprecht et al. (2009) [20] haben wir für zwei der typischen Trockenrasenassoziationen der Region, das Stipetum pulcherrimae und das Stipetum lessingianae (die nomenklatorische Prüfung dieser Namen steht noch aus), untersucht, wie sich eine auf den einzelnen Flächen 30-50 Jahre zurückliegende Nutzungsänderung auf die heutige Artenzusammensetzung und auf Diversitätsparameter auswirkt. Da keine Vegetationsaufnahmen aus der Zeit vor der Nutzungsänderung vorliegen, haben wir stattdessen einen Vergleich mit aktuell noch beweideten Trockenrasen in ähnlicher standörtlicher Situation vorgenommen (space-for-time substitution). Um Vergleichbarkeit der Vegetationsaufnahmen, die teils auf $16 \mathrm{~m}^{2}$, teils auf $25 \mathrm{~m}^{2}$ angefertigt worden waren, zu gewährleisten, haben wir alle Artenreichtumsdaten mit $z=0,21$ auf $16 \mathrm{~m}^{2}$ standardisiert (Dengler 2005 [15]). Es zeigte sich, dass sich die Bestandesstruktur in beiden Assoziationen signifikant und stark geändert hat, d. h. die Deckung der Krautschicht in den Pinus-Plantagen war erheblich reduziert, die Deckung der Streu hatte von den genutzten über die brach gefallenen hin zu den aufgeforsteten Beständen zugenommen und nennenswerte Anteile von offenem Boden gab es nur in den noch genutzten Beständen (Ruprecht et al. 2009 [20]). Die standardisierten Artenzahlen nahmen im Stipetum lessingianae von der Weide über die Brache hin zur Aufforstung leicht und nicht signifikant ab, während im Stipetum pulcherrimae (wo es keine noch genutzten Bestände gab), die Aufforstungen signifikant artenreicher waren als die Brachen. Alle anderen betrachteten Diversitätsmaße (Shannon-Index, Evenness, Anzahl Rote-Liste-Arten) zeigten dagegen keinen signifikanten Effekt der verschiedenen Nutzungen. Während die Veränderungen auf der Ebene summarischer Diversitätsparameter also gering ausfielen, zeigte sich bei der Betrachtung von Einzelarten, dass v. a. typische Festuco-Brometea-Arten (z. B. Dichantium ischaemum und Asperula cynanchica) 
bei Aufforstung stark zurückgehen, während sich weit verbreitete, ruderale oder holzige Arten ausbreiten.

In Bulgarien geht die extensive Weidenutzung artenreicher Grasländer ebenfalls erheblich zurück, insbesondere seit Beitritt des Landes zur Europäischen Union. Um den Effekt der Nutzungsaufgabe auf die pflanzliche Biodiversität zu analysieren haben wir in einem Abschnitt des Balkangebirges (Ponor) die Gefäßpflanzendiversität von 137 zufällig platzierten $16 \mathrm{~m}^{2}$-Plots ebenfalls mit einer space-for-time substitution analysiert (Vassilev et al. 2011 [21]; siehe Abb. 20). Teils werden die Flächen noch beweidet, teils waren sie in den 1990er Jahren brach gefallen.

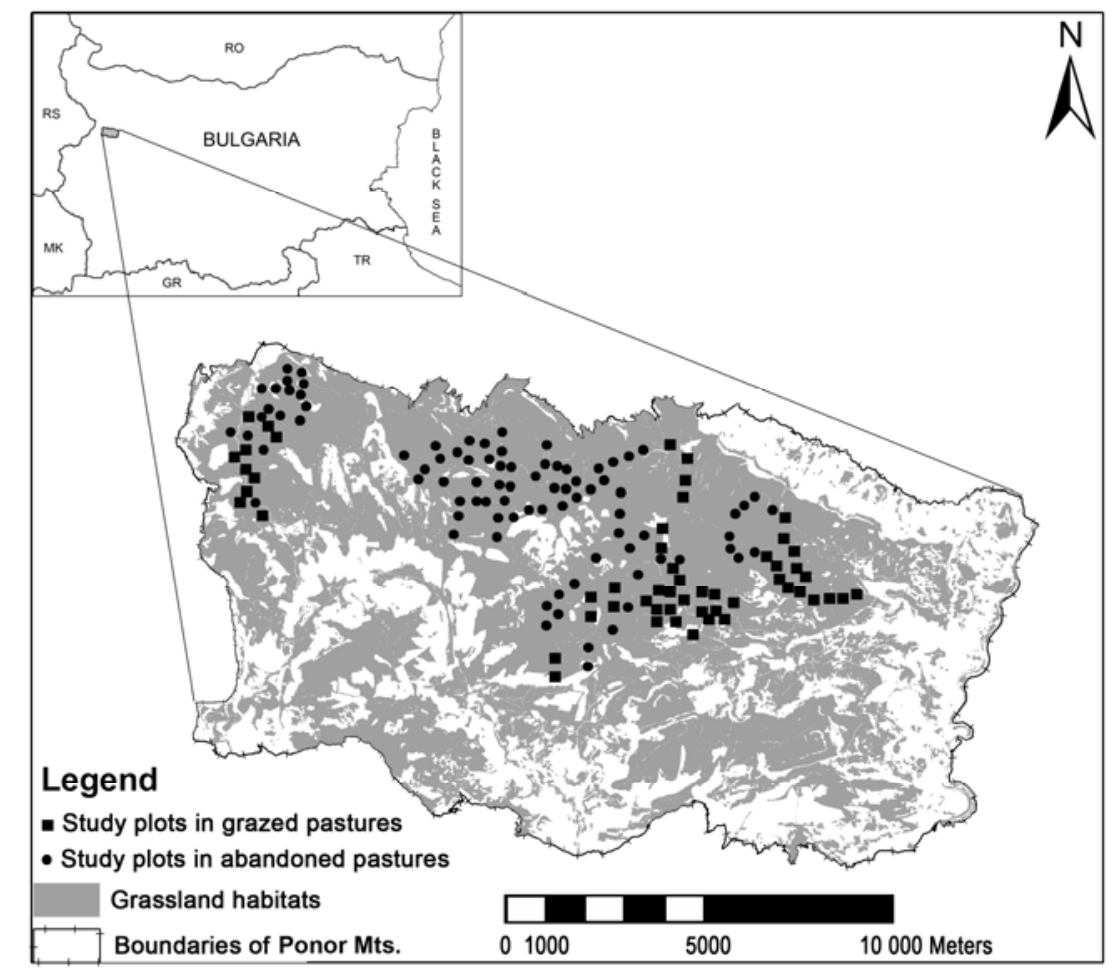

Abb. 20: Lage des Untersuchungsgebietes und der Aufnahmeflächen in der Studie zum Einfluss der Nutzungaufgabe auf die Gefäßpflanzendiversität bulgarischer Grasländer (aus Vassilev et al. 2011 [21]). Die graue Fläche bezeichnet die Grasländer, die schwarzen Quadrate beweidete Plots und die schwarzen Kreis brach gefallene Plots.

Es zeigte sich, dass die Nutzungsaufgabe vermehrt Flächen in größerer Höhenlage und auf tiefgründigeren Böden betraf. Deshalb wurden diese beiden abiotischen Parameter als Covariablen der Kovarianzanalysen (ANCOVAs) verschiedener Diversitäts- und Strukturparameter genutzt (Tab. 6). Zwar nahm die Gesamtartenzahl beim Brachfallen erwartungsgemäß ab, doch stieg zugleich die Zahl der Arten mit Naturschutzbedeutung sogar leicht an. Weiterhin zeigte sich, dass auch rund 15 Jahren nach dem Brachfallen, die Sukzession in Richtung Gebüsch extrem 
langsam erfolgt, diese zoo-anthropogenen Graslandgesellschaften also unerwartet stabil sind. Tatsächlich wuchsen auf den beweideten Flächen sogar geringfügig, aber signifikant mehr junge Gehölze, was darauf zurückzuführen sein könnte, dass die Störungen durch die Weidetiere erst geeignete Etablierungsnischen schaffen.

Tab. 6: Unterschiede in Diversitäts- und Strukturparametern zwischen beweideten und brach gefallenen Trockenrasen im Balkangebirge (Bulgarien) (verändert aus Vassilev et al. 2011 [21]). Dargestellt ist der tatsächliche Mittelwerte (d. h. ohne Standardisierung durch die Covariablen) \pm Standardabweichung. Der $p$-Wert bezieht sich auf ANCOVAs mit Meereshöhe und Tiefgründigkeit als Covariablen.

\begin{tabular}{lccr}
\hline Parameter & Weide & Brache & $\boldsymbol{p}$-Wert \\
\hline Artenreichtum Gefäßpflanzen $\left(16 \mathrm{~m}^{2}\right)$ & $\mathbf{3 4 , 3 \pm \mathbf { 8 , 1 }}$ & $32,3 \pm 8,7$ & $\mathbf{0 , 0 0 2}$ \\
Anzahl von Arten mit Naturschutzbedeutung $\left(16 \mathrm{~m}^{2}\right)$ & $1,0 \pm 1,2$ & $\mathbf{1 , 7} \pm \mathbf{1 , 3}$ & $<\mathbf{0 , 0 0 1}$ \\
Vegetationshöhe [cm] & $61,1 \pm 20,4$ & $\mathbf{7 4 , 4} \pm \mathbf{2 7 , 1}$ & $<\mathbf{0 , 0 0 1}$ \\
Deckung Gehölze [\%] & $\mathbf{4 , 2} \pm 5,7$ & $2,6 \pm 4,6$ & $\mathbf{0 , 0 3 2}$ \\
\hline
\end{tabular}

Wie schon im rumänischen Beispiel zeigte sich auch in Ponor, dass recht geringen Änderungen der Diversitätsparameter erhebliche Veränderungen in der Artenzusammensetzung gegenüber stehen. Von den insgesamt 316 Gefäßpflanzenarten waren 33 in den Brachen signifikant seltener und 21 signifikant häufiger. Die erste Gruppe umfasst v. a. typische Festuco-Brometea-Arten und einige allgemeine Weidezeiger (wie Disteln), die zweite v. a. Trifolio-Geranietea-Arten und einige mesophile Gräser. Bei der Betrachtung des Reichtums in verschiedenen funktionellen Gruppen zeigten sich die größten Unterschiede bezüglich der Blattanordnung (Rosettenpflanzen bei Beweidung angerreichert, Arten mit Beblätterung an der ganzen Sprossachse bei Brache angereichert) und der hygrischen Präferenz von Arten (Mesoxerophyten und Xerophyten bei Beweidung angereichert, Mesophyten dagegen bei Brache). Die Befunde zeigen insgesamt große Ähnlichkeiten mit anderen Studien im südöstlichen Europa (z. B. Ruprecht et al. 2009 [20], Škornik et al. 2010), aber auch einige gebietsspezifische Besonderheiten. Wir kommen zu dem Schluss, dass es für die Erhaltung der botanischen Diversität des Gebietes insgesamt am besten ist, wenn man versucht, ein Mosaik von unterschiedlichen Nutzungsintensitäten der Grasländer von relativ stark beweidet bis zeitweise ungenutzt - aufrechtzuerhalten.

In Friedel et al. (2006) [22] schließlich untersuchen wir in nordostdeutschen Buchenwäldern den Einfluss der Waldbewirtschaftung auf die Diversität und Artenzusammensetzung epiphytischer Moose und Flechten. Dazu wurden je 100 Buchen (Fagus sylvatica) der oberen Baumschicht in einem seit 1961 nicht mehr forstwirtschaftlich genutzten Waldschutzgebiet (Serrahn) und in einem nahe gelegenen und vergleichbaren Nutzwald (Wilhelminenhof) analysiert. Die Erfassung aller Moos- und Flechtenarten erfolgte bis zu einer Stammhöhe von $2 \mathrm{~m}$. Da sich die 
Stammdurchmesser und damit die besiedelbaren Rindenflächen in beiden Waldtypen erheblich unterschieden, haben wir die Artenzahlen für den Vergleich mittels einer Artenzahl-Areal-Kurve (siehe Abschnitte 1.5 und 3.1) auf $1 \mathrm{~m}^{2}$ standardisiert. Dabei erwies sich eine Potenzfunktion mit $z=0,459$ als das beste Modell. Es zeigte sich, dass die Gesamtzahl der auf 100 Bäumen gefundenen Epiphyten im urwaldähnlichen Serrahn zwar 40 \% höher war als im Wirtschaftswald, dass der Unterschied bezogen auf die gleiche Rindenfläche deutlich geringer ausfällt und nur bei den Flechten signifikant ist (Tab. 7).

Tab. 7: Vergleich der Epiphytenflora von je 100 Buchenstämmen in einem unbewirtschafteten und einem bewirtschafteten Buchenwald in Mecklenburg-Vorpommern (verändert aus Friedel et al. 2006 [22]). Es sind die Gesamtwerte für 100 Stämme und die auf $1 \mathrm{~m}^{2}$ Rindenfläche standardisierten Werte dargestellt. Fettsatz zeigt einen signifikanten Unterschied auf dem Signifkanzniveau $\alpha=0,05$ an.

\begin{tabular}{lcc}
\hline & $\begin{array}{c}\text { Unbewirtschafteter } \\
\text { Wald }\end{array}$ & Wirtschaftswald \\
\hline Untersuchte Stammoberfläche $\left[\left(\mathrm{m}^{2}\right]\right.$ & 345 & 296 \\
Gesamtartenzahl Moose & 27 & 18 \\
Gesamtartenzahl Flechten & 22 & 17 \\
Mittlere Artendichte Moose $\left(1 \mathrm{~m}^{2}\right)$ & $1,7 \pm 1,2$ & $1,5 \pm 0,9$ \\
Mittlere Artendichte Flechten $\left(1 \mathrm{~m}^{2}\right)$ & $\mathbf{1 , 1} \pm \mathbf{1 , 0}$ & $\mathbf{0 , 8} \pm \mathbf{0 , 9}$ \\
\hline
\end{tabular}

Wirft man einen näheren Blick auf die Artenzusammensetzung, zeigen sich sehr viel deutlichere Unterschiede zwischen den Nutzungsvarianten. Fünf Epiphytenarten waren auf Bäumen des ungenutzten Waldes signifikant häufiger (u. a. die Flechten Graphis scripta und Pyrenula nitida), während vier im Nutzwald häufiger vertreten waren (u. a. das Moos Aulacomnium androgynum). Betrachtet man die Zeigerwerte der beiden Gruppen diagnostischer Arten (Tab. 8), so zeigt sich, dass die typischen Arten des Nutzwaldes höheren Lichtgenuss, saurere Rinde und stärkeren Schadstoffeinfluss anzeigen, wobei nur der erste Unterschied signifikant war.

Tab. 8: Vergleich der ökologischen Zeigerwerte der diagnostischen Epiphytenarten für unbewirtschaftete und bewirtschaftete Buchenwälder in Mecklenburg-Vorpommern (verändert aus Friedel et al. 2006 [22]). In Klammern ist jeweils die Zahl der diagnostischen Arten mit verfügbaren Zeigerwerten angegeben. Fettsatz zeigt einen signifikanten Unterschied auf dem Signifkanzniveau $\alpha=0,05$ an.

\begin{tabular}{lcccc}
\hline & Licht & Feuchte & Reaktion & Toxitoleranz \\
\hline Indikatorarten für ungenutzten Wald & $\mathbf{3 , 4}(n=5)$ & $4,0(n=5)$ & $4,3(n=4)$ & $5,4(n=5)$ \\
Indikatorarten für genutzten Wald & $\mathbf{5 , 5 ( n = 4 )}$ & $4,5(n=4)$ & $2,5(n=4)$ & $7,3(n=3)$ \\
\hline
\end{tabular}




\subsection{Großräumige Biodiversitätsmuster im südlichen Afrika}

Die im Rahmen des Projektes BIOTA Southern Africa erhobenen Daten zur Gefäßpflanzendiversität auf standardisierten BIOTA Biodiversitätsobservatorien (Jürgens et al. im Druck [2]) bieten die Chance, Phytodiversitätsmuster entlang zweier zusammen über 2000 km langen Transekte im Detail zu beschreiben und durch die gemessenen oder modellierten Umweltfaktoren zu erklären. Hier handelt es sich also um großräumige (bezogen auf den extent) oder makroökologische Muster. In Schmiedel et al. (2010) [23] präsentieren wir erste Auswertungen dieses auch auf globaler Skala hinsichtlich Methodenkonsistenz und großräumiger Abdeckung einmaligen Datensatzes. Hervorzuheben ist zudem die große Zahl verfügbarer Biodiversitäts- und abiotischen Umweltparameter (Tab. 9).

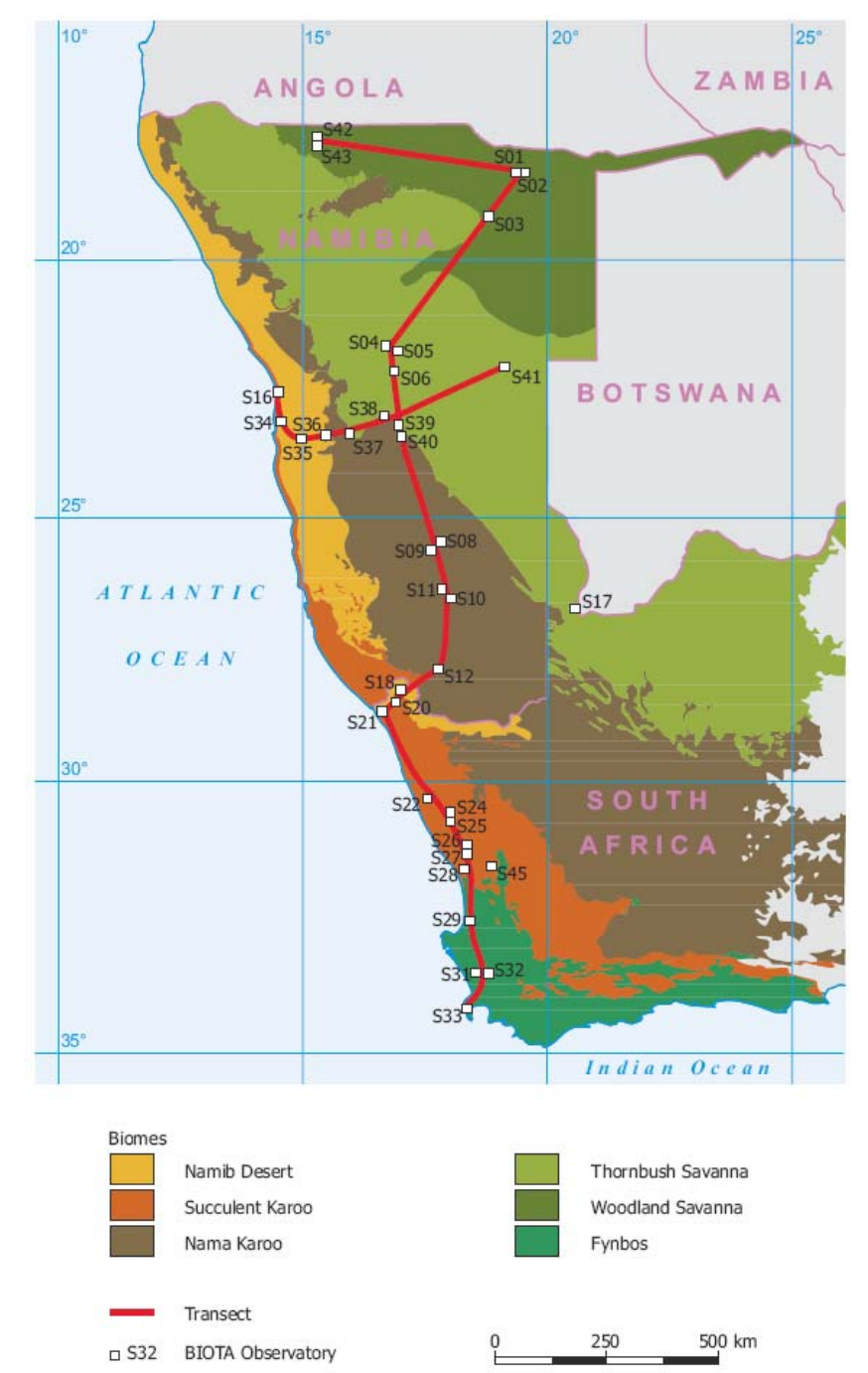

Abb. 21: Lage der 37 BIOTA-Biodiversitätsobservatorien (Quadrate) entlang der Transekte von BIOTA Southern Africa mit Zuordnung zu den sechs Biomen der Region (aus Haarmeyer et al. 2010b). Die Biome sind von Süd nach Nord: Fynbos, Sukkulente Karoo, Nama Karoo, Dornbuschsavanne und Baumsavanne, sowie entlang der namibianischen Küste die Namibwüste. 
Tab. 9: Variablen, die in den statistischen Analysen der Muster der Gefäßpflanzendiversität im südlichen Afrika genutzt wurden. Überwiegend handelt es sich um metrische Variablen; die ordinalen und kategorialen Variablen sowie evtl. Transformationen metrischer Daten zur Erzielung ungefährer Normalverteilung sind durch entsprechende Zusätze in eckigen Klammern gekennzeichnet (verändert aus Schmiedel et al. 2010 [23]).

\begin{tabular}{|c|c|c|}
\hline Variablentyp & Variablengruppe & Variablen \\
\hline \multirow[t]{5}{*}{ Prediktoren } & Allgemeine Daten & $\begin{array}{l}\text { Geografische Breite; geografische Länge; Meeres- } \\
\text { höhe; Regensaison [2 Kategorien]; Biom [6 Katego- } \\
\text { rien], Intensität der Landnutzung [4-teilige Ordi- } \\
\text { nalskala, als quasi-metrisch behandelt] }\end{array}$ \\
\hline & $\begin{array}{l}\text { Klima (modellierte Daten aus } \\
\text { Worldclim, Hijmans et al. } \\
\text { 2005) }\end{array}$ & $\begin{array}{l}\text { Jahresmitteltemperatur; Jahresminimaltemperatur; } \\
\text { Jahresmaximaltemperatur; jährliche Temperatur- } \\
\text { spanne; tägliche Temperaturspanne; Jahresnieder- } \\
\text { schlag; Anteil des Winterregens; Dauer der Tro- } \\
\text { ckensaison; Ariditätsindex (De Martonne) }\end{array}$ \\
\hline & $\begin{array}{l}\text { Klima (Daten von den BIO- } \\
\text { TA-Wetterstationen) }\end{array}$ & $\begin{array}{l}\text { Interannuelle Niederschlagsvariabilität; relative } \\
\text { Luftfeuchtigkeit; Windgeschwindigkeit; Global- } \\
\text { strahlung; Potenzielle Evapotranspiration (PET); } \\
\text { Ariditätsindex (UNEP) }\end{array}$ \\
\hline & Boden (Chemie) & $\begin{array}{l}\mathrm{pH} \text {; elektrische Leitfähigkeit [log-transformiert]; } \\
\text { organischer Kohlenstoffgehalt [log-transformiert] }\end{array}$ \\
\hline & $\begin{array}{l}\text { Boden (Pedodiversität, nach- } \\
\text { Petersen 2008) }\end{array}$ & $\begin{array}{l}\text { Anzahl an Bodenreferenzgruppen;Anzahl an Bo- } \\
\text { deneinheiten; Evenness der Bodeneinheiten; Anzahl } \\
\text { der Bodenökotypen; Größe des parametrischen } \\
\text { Raumes }\end{array}$ \\
\hline \multirow[t]{2}{*}{$\begin{array}{l}\text { Biodiversitätsparameter } \\
\text { (abhängige Variablen) }\end{array}$} & $\alpha$-Diversität & $\begin{array}{l}\text { Kumulierte Familienzahl }\left(1 \mathrm{~km}^{2}\right) \text {; kumulierte Arten- } \\
\text { zahl }\left(1 \mathrm{~km}^{2}\right) \text {; Artenreichtum }\left(1 \mathrm{~km}^{2} \text {, ausgewähltes }\right. \\
\text { Jahr }) \text {; mittlerer Artenreichtum }\left(1000 \mathrm{~m}^{2}\right) \text {; maximaler } \\
\text { Artenreichtum }\left(1000 \mathrm{~m}^{2}\right) \text {; mittlerer Artenreichtum } \\
\left(100 \mathrm{~m}^{2}\right) \text {; maximaler Artenreichtum }\left(100 \mathrm{~m}^{2}\right)\end{array}$ \\
\hline & $\beta$-Diversität & $\begin{array}{l}z \text {-Wert }\left(1000 \mathrm{~m}^{2}-1 \mathrm{~km}^{2}\right) ; z \text {-Wert }\left(100-1000 \mathrm{~m}^{2},\right. \\
\text { berechnet für zusammengehörige Werte aus nested } \\
\text { plots }) ; z \text {-Wert }\left(100-1000 \mathrm{~m}^{2} \text {; berechnet für die }\right. \\
\text { Mittelwerte des jeweiligen Observatoriums })\end{array}$ \\
\hline
\end{tabular}

Es zeigte sich das die Gefäßpflanzendiversität erheblich zwischen den sechs Biomen variiert (Abb. 22). Das Muster zwischen den Biomen (d. h. ihre Diversität relativ zu den anderen) ändert sich zudem maßgeblich in Abhängigkeit von der räumlichen Hierarchieebene $(\alpha-$ bzw. $\beta$ Diversität; vgl. Abschnitt 1.4) und der betrachteten räumlichen $\left(100 \mathrm{~m}^{2}, 1000 \mathrm{~m}^{2}, 1 \mathrm{~km}^{2}\right)$ und taxonomischen Skala (Arten vs. Familien; vgl. Abschnitt 1.5). So ist etwa die $\alpha$-Diversität in der Namibwüste am niedrigsten, die $\beta$-Diversität (gemessen als $z$-Wert der Artenzahl-ArealBeziehung) dagegen am höchsten und zugleich am variabelsten. Beim Artenreichtum liegt der Fynbos unter den betrachteten Biomen auf den beiden kleinen Skalenebenen weit vorne, während auf $1 \mathrm{~km}^{2}$ die Observatorien in der Sukkulenten Karoo ähnlich artenreich waren (im Median sogar artenreicher). Dieser Befund stellt eine Grundannahme der Makroökologie in Frage, dass nämlich latitudinale Artendichtegradienen ,universell“ seien (z. B. Kaufman 1995, Rosenzweig 1995, Gaston \& Spicer 2004). Sie sind es offensichtlich nicht - je nach betrachter Skalenebene 
können sich die Muster erheblich ändern (vgl. dazu auch ähnliche Befunde aus einem gänzlich anderen Ökosystem in Dengler et al. 2006b [31] und Dengler \& Boch 2008a [36]). Das Bild ändert sich auch bei der Betrachtung verschiedener taxonomischer Skalen erheblich: so ist die Baumsavanne auf $1 \mathrm{~km}^{2}$ beim Artenreichtum nur im Mittelfeld, übertrifft beim Familienreichtum dann aber sogar die Sukkulente Karoo und den Fynbos.
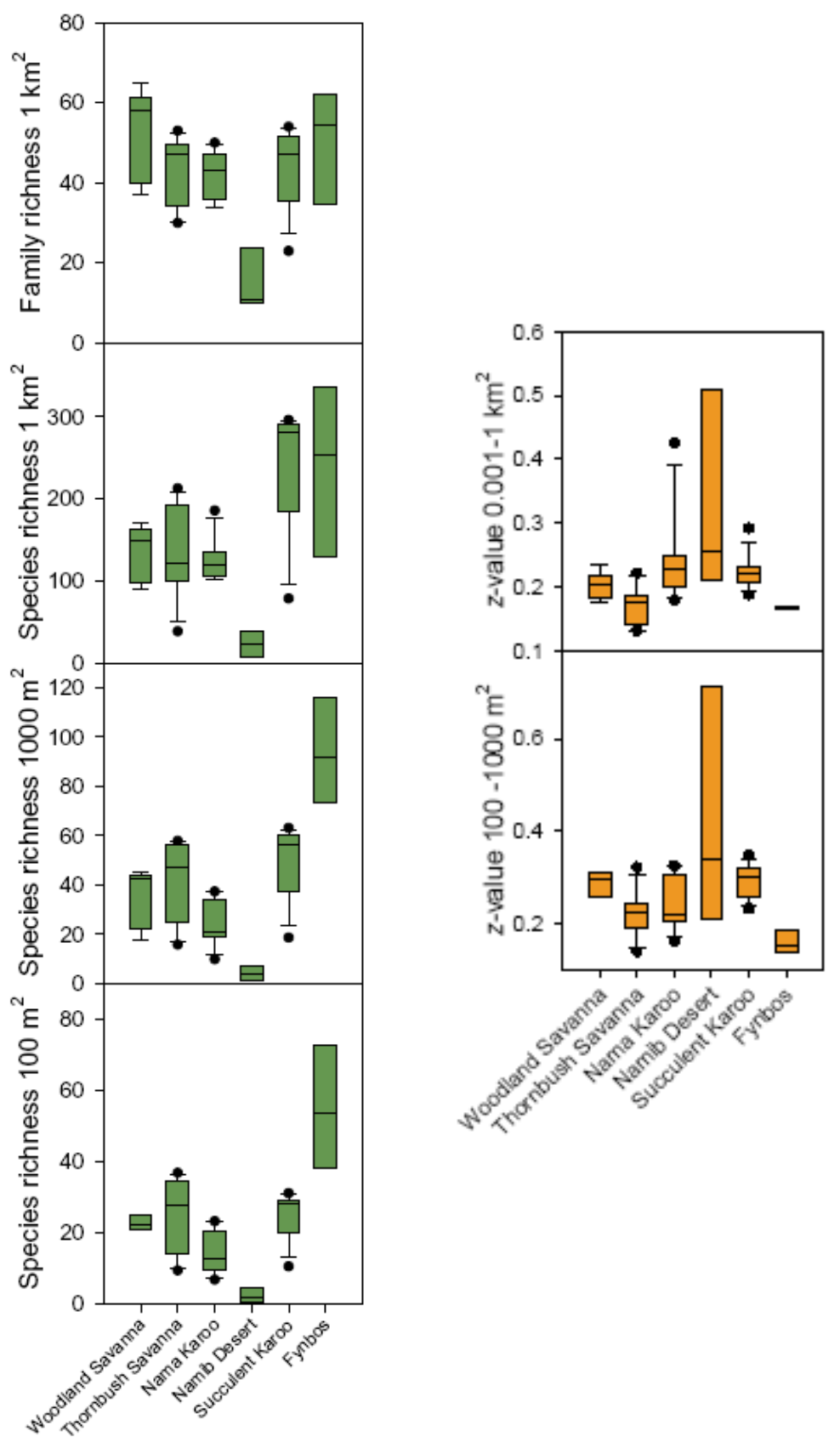

Abb. 22: Vergleich von vier $\alpha$-Diversitätsmaßen (links) und zwei $\beta$-Diversitätsmaßen (rechts) in den sechs Biomen im westlichen Teil des südlichen Afrikas (aus Schmiedel et al. 2010 [23]). 
In einer ersten Analyse der Faktoren, die Einfluss auf die verschiedenen Biodiversitätsparameter nehmen (detaillierte Analysen sind derzeit in Arbeit), zeigte sich dass die beiden wichtigsten Faktoren die Niederschlagsmenge und die Niederschlagsverteilung innerhalb des Jahres sind (Abb. 23), wobei die Winterregengebiete auf allen Skalenebenen ungefähr 2,5 mal so viele Arten wie die Sommerregengebiete aufweisen. Während der positive Einfluss der Niederschlagsmenge auf Biodiversität in der Makroökologie allgemein anerkannt ist (Kreft et al. 2008, Qian \& Ricklefs 2008), kennen wir keine andere makroökologische Studie, die zuvor einen solchen Einfluss der Art der Saisonalität (nicht des Grades der Saisonalität) demonstriert hat. Dies stellt die Makroökologie vor die Herausforderung, zu testen, ob dieses Muster auch in anderen Erdregionen und auch für andere Großtaxa gilt und was mögliche Erklärungen sind.

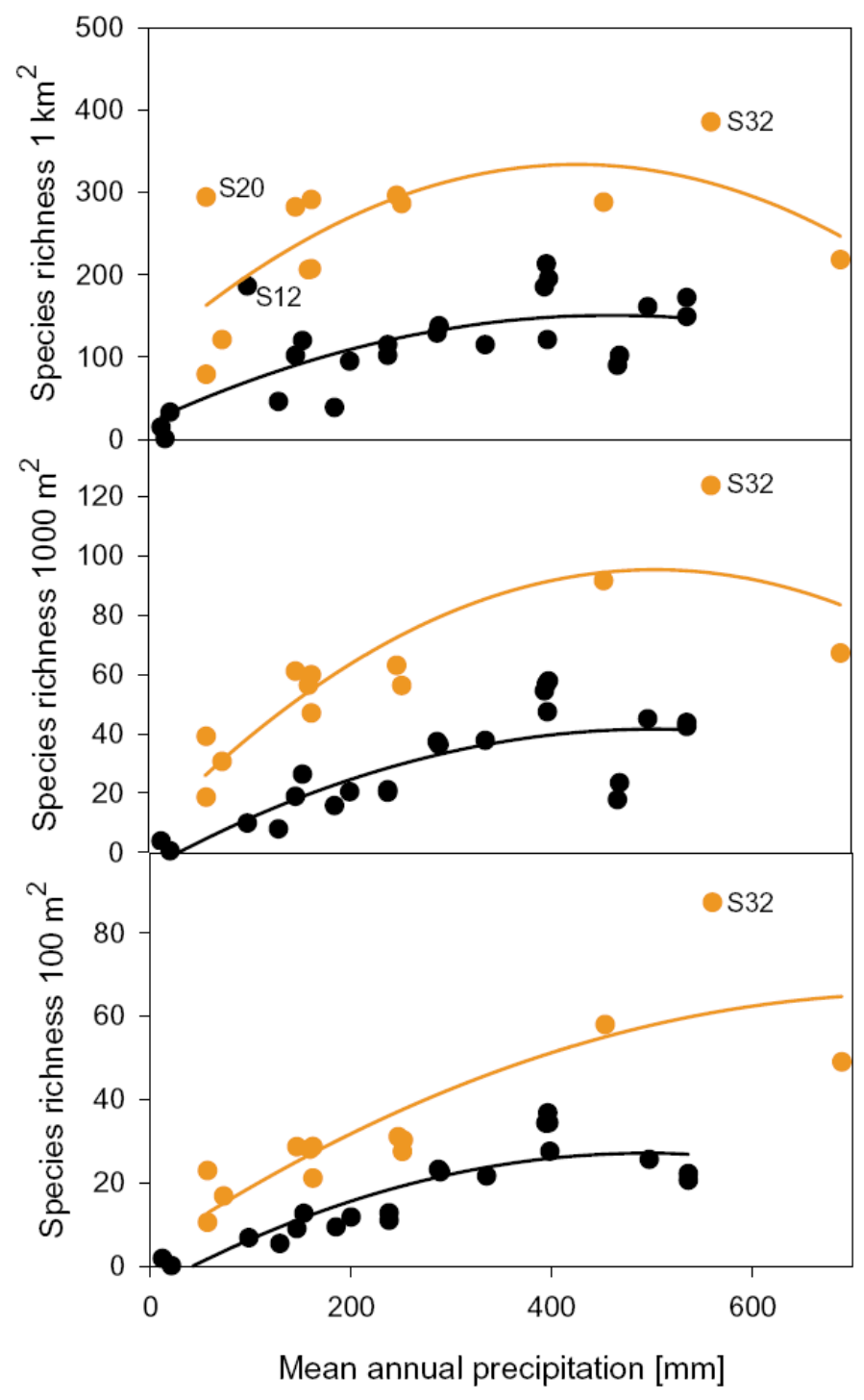

Abb. 23: Einfluss von Niederschlagsmenge und Niederschlagsverteilung auf den Gefäßpflanzenartenreichtum im südlichen Afrika, dargestellt für drei Korngrößen (grain sizes) (aus Schmiedel et al. 2010 [23]). Die obere Kurve bezeichnet jeweils die Winterregengebiete, die untere die Sommerregengebiete. 
Unter den Bodenparametern hatte der $\mathrm{pH}-$ Wert einen negativen Einfluss auf den Artenreichtum auf $1000 \mathrm{~m}^{2}(p=0,026)$, also genau anders herum als in den meisten temperaten und borealen Ökosystemen (vgl. Abschnitt 4.3). Auf der Quadratkilometer-Skala wurde der Gefäßpflanzenartenreichtum stark und hochsignifikant von der Anzahl der Bodenökotypen beeinflusst, die auf einem Observatorium vorkamen ( $p=0,003 ; r^{2}=0,3616$; Abb. 24).

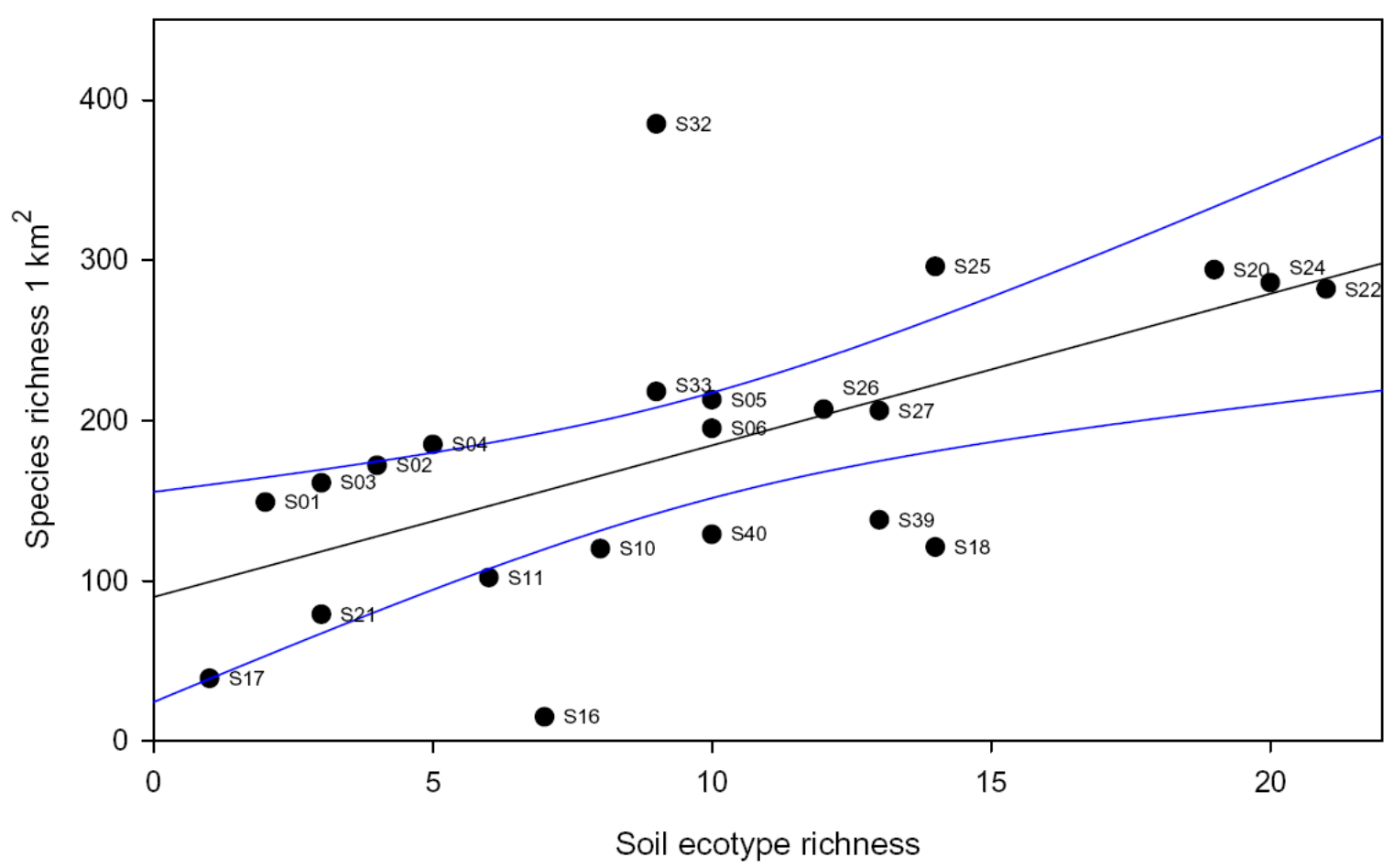

Abb. 24: Einfluss der Anzahl der Bodenökotypen auf den Gefäßpflanzenartenreichtum pro $1 \mathrm{~km}^{2}$ (aus Schmiedel et al. 2010 [23]).

\subsection{Kleinräumige Biodiversitätsmuster im südlichen Afrika}

Zwei Studien mit meiner Beteiligung beschäftigen sich mit den kleinräumigen (bezogen den extent) Biodiversitätsmuster in der Sukkulenten Karoo, demjenigen Biom im südlichen Afrika, das als einziges arides Biom zu den 25 globalen biodiversity hotspots von Myers et al. (2000) gehört. Haarmeyer et al. (2010a) [24] betrachten dabei den Einfluss von Habitat und Nutzungsintensität, während Schmiedel et al. (im Druck) [25] die zeitliche Dynamik über mehr als eine Dekade analysieren. Beide Studien fanden im Zentrum der Knersvlakte statt (Abb. 25). 


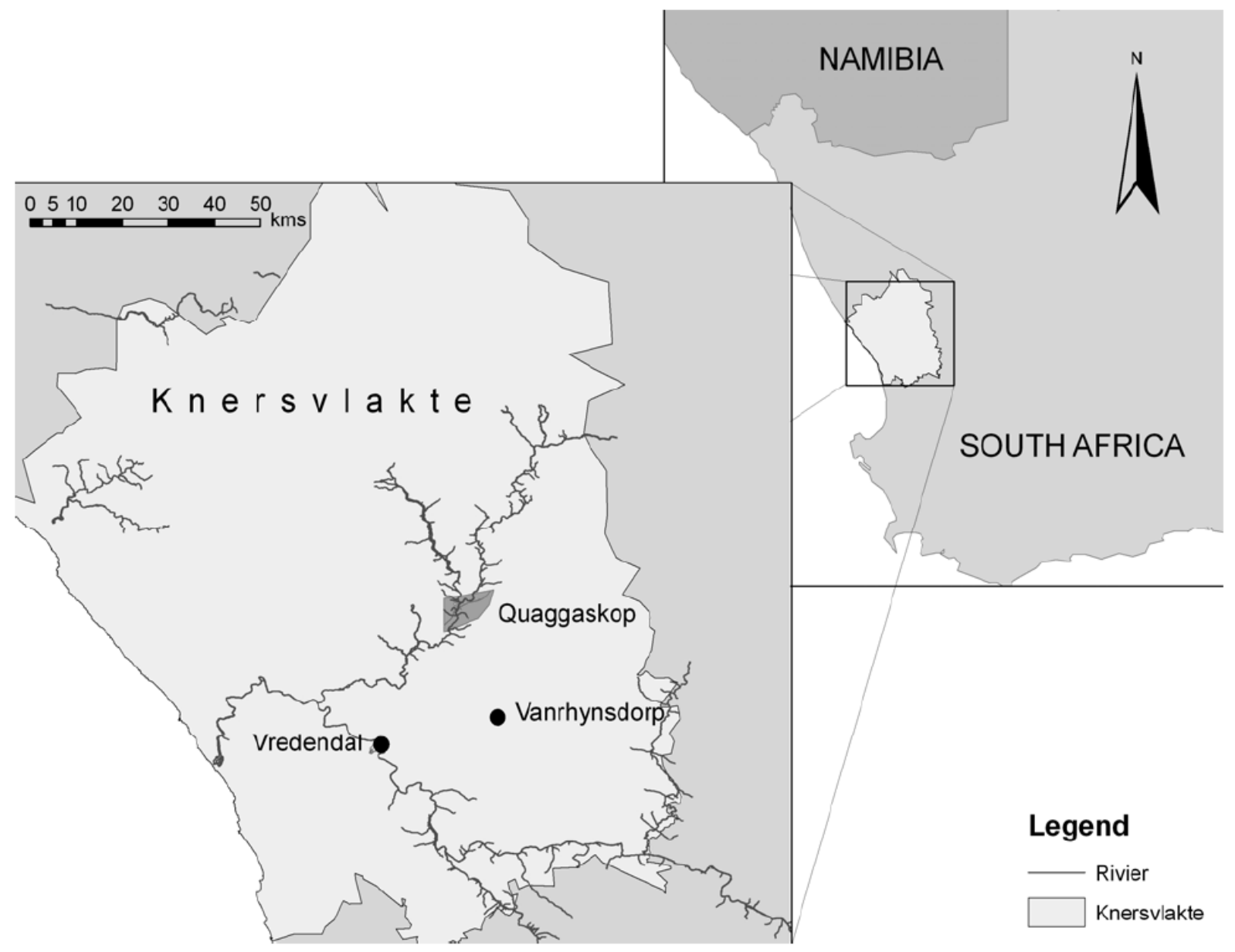

Abb. 25: Lage der Farm Quaggaskop in der Knersvlakte, auf der die Studien von Haarmeyer et al. (2010a; incl. benachbarter Farmen) [24] und Schmiedel et al. (im Druck) [25] stattfanden (aus Schmiedel et al. im Druck [25]).

Im Zuge der Erstellung eines Managementplanes für die geplante Knersvlakte Conservation Area haben Haarmeyer et al. (2010a) [24] den Effekt verschiedener Beweidungsintensitäten mit Schafen und Ziegen auf naturschutzrelevante Parameter auf der Populationsebene einzelner Pflanzenarten (Fortpflanzungserfolg, Keimungserfolg - hier nicht wiedergegeben) und auf der Ebene der Pflanzengesellschaft (Biodiversitätsindizes, Artenzusammensetzung) analysiert. Dazu wurden pro Beweidungsintensität (Kategorien: keine, mittel, hoch) verteilt über verschiedene Farmen und Camps je 17 Plots von $1000 \mathrm{~m}^{2}(50 \mathrm{~m} \times 20 \mathrm{~m})$ angelegt, auf denen dann die eigentlichen Untersuchungen auf 100 regelhaft angeordneten Probeflächen (Subplots) von je $400 \mathrm{~cm}^{2}$ stattfanden. Dieses Design erlaubt es, $\alpha$-, $\beta$ - und $\gamma$-Komponenten der Diversität (vgl. Abschnitt 1.4) getrennt zu betrachten, wobei als Surrogat für die $\gamma$-Diversität die kumulierte Artenzahl aller zugehörigen 100 Subplots $(\alpha)$ verwendet wurde.

Es zeigte sich, dass sich die beiden im Gebiet vorkommenden Habitattypen Quarzfelder (siehe Schmiedel et al. im Druck [25]) und die dazwischen liegende Matrix mit meist lehmigen Bö- 
den in fast allen Biodiversitätsparametern wie auch in den gemessenen Umweltparametern erheblich unterscheiden (Auswahl in Tab. 10). Während die Matrix auf beiden Skalenebenen $(\alpha, \gamma)$ erheblich artenreicher ist, kommen in den Quarzfeldern trotzdem mehr endemische Arten vor und $\beta$-Diversität sowie Evenness unterscheiden sich nicht signifikant. Dieser erhebliche Unterschied zwischen den beiden Habitattypen machte es erforderlich, den Beweidungseffekt für jedes der beiden Habitate getrennt zu betrachten (Tab. 11, 11). Es zeigt sich, dass in beiden Habitaten der kleinräumige Artenreichtum ohne Beweidung am höchsten ist. Während auf den Quarzfeldern die übrigen Diversitätsparameter nicht signifikant von der Beweidung beeinflusst werden, verhalten sich $\beta$-Diversität und Evenness in der Matrixvegetation unimodal in Relation zur Beweidungsintensität. Die entspricht den Vorhersagen der Intermediate Disturbance Hypothesis (IDH; vgl. Connell 1978). Dagegen ist die $\alpha$-Diversität der Matrixvegetation bei mittlerer Beweidungsintensität geringer als bei fehlender wie auch bei starker Beweidung. Dieses Muster ist unvereinbar mit der IDH und wir konnten auch sonst keine überzeugende Erklärung dafür finden. Schließlich haben wir auch noch den Landnutzungseffekt auf die Artenzusammensetzung analysiert, indem wir diagnostische Arten mittels phi-Werten (Chytrý et al. 2002) für die drei Beweidungsstufen innerhalb der beiden Habitattypen bestimmt haben. Es zeigte sich in beiden Habitaten, dass es umso mehr diagnostische Arten gibt, je niedriger die Beweidungsstufe ist, wobei endemische Arten überhaupt nur diagnostisch für keine Beweidung oder moderate Beweidung waren.

Tab. 10: Vergleich ausgewählter Biodiversitätsindizes (Gefäßpflanzen) und Umweltparameter zwischen den beiden Habitattypen der Knersvlakte, Quarzfeld und Matrix, mit $t$-Tests (verändert aus Haarmeyer et al. 2010a [24]). Es sind jeweils die Mittelwerte \pm Standardabweichungen angegeben.

\begin{tabular}{lccl}
\hline Variable & Quarzfeld & Matrix & $\boldsymbol{p}$-Wert \\
\hline$\alpha$-Diversität: mittlere Artenzahl pro Subplot $\left(0.04 \mathrm{~m}^{2}\right)$ & $1,14 \pm 0,47$ & $1,66 \pm 0,62$ & $\mathbf{0 , 0 0 2}$ \\
$\gamma$-Diversität: kumulierte Artenzahl per Plot $\left(1000 \mathrm{~m}^{2}\right)$ & $23 \pm 6$ & $31 \pm 6$ & $<\mathbf{0 , 0 0 1}$ \\
$\beta$-Diversität $($ multiplikativ) & $22 \pm 7$ & $21 \pm 6$ & 0,439 \\
Shannon-Evenness $\left(1000 \mathrm{~m}^{2}\right)$ & $0,46 \pm 0,12$ & $0,42 \pm 0,17$ & 0,335 \\
Anzahl endemischer Arten $\left(1000 \mathrm{~m}^{2}\right)$ & $8 \pm 3$ & $6 \pm 2$ & $\mathbf{0 , 0 1 3}$ \\
\hline $\mathrm{pH}$ & $6,33 \pm 1,31$ & $7,67 \pm 0.47$ & $<\mathbf{0 , 0 0 1}$ \\
Leitfähigkeit $\left[\mu \mathrm{S} \mathrm{cm}^{-1}\right], \log$-transformiert & $4836 \pm 2405$ & $2554 \pm 2117$ & $<\mathbf{0 , 0 0 1}$ \\
\hline
\end{tabular}


Tab. 11: Vergleich ausgewählter Biodiversitätsindizes (Gefäßpflanzen) in Quarzfeldern der Knersvlakte zwischen den drei Beweidungsstufen mittels ANOVAs (verändert aus Haarmeyer et al. 2010a [24]). Es sind jeweils die Mittelwerte \pm Standardabweichungen angegeben. Die hochgestellten Buchstaben bezeichnen homogene Gruppen gemäß Tukeys HSD post-hoc-Test auf einem Signifikanzniveau von $\alpha=0,05$.

\begin{tabular}{lcccc}
\hline Variable & Keine & Mittel & Hoch & $\boldsymbol{p}$-Wert \\
\hline$\alpha$-Diversität: mittlere Artenzahl pro Subplot $\left(0.04 \mathrm{~m}^{2}\right)$ & $1,51 \pm 0,41^{\mathrm{a}}$ & $0,92 \pm 0,30^{\mathrm{b}}$ & $1,00 \pm 0,47^{\mathrm{b}}$ & $\mathbf{0 , 0 0 9}$ \\
$\gamma$-Diversität: kumulierte Artenzahl per Plot $\left(1000 \mathrm{~m}^{2}\right)$ & $26 \pm 6$ & $21 \pm 6$ & $22 \pm 7$ & 0,282 \\
$\beta$-Diversität (multiplikativ) & $18 \pm 5$ & $24 \pm 7$ & $24 \pm 8$ & 0,086 \\
Shannon-Evenness $\left(1000 \mathrm{~m}^{2}\right)$ & $0,41 \pm 0,10$ & $0,51 \pm 0,11$ & $0,46 \pm 0,14$ & 0,190 \\
Anzahl endemischer Arten $\left(1000 \mathrm{~m}^{2}\right)$ & $9 \pm 3$ & $9 \pm 3$ & $6 \pm 3$ & 0,177 \\
\hline
\end{tabular}

Tab. 12: Vergleich ausgewählter Biodiversitätsindizes (Gefäßpflanzen) in der Matrix der Knersvlakte zwischen den drei Beweidungsstufen mittels ANOVAs (verändert aus Haarmeyer et al. 2010a [24]). Es sind jeweils die Mittelwerte \pm Standardabweichungen angegeben. Die hochgestellten Buchstaben bezeichnen homogene Gruppen gemäß Tukeys HSD post-hoc-Test auf einem Signifikanzniveau von $\alpha=0,05$.

\begin{tabular}{lcccc}
\hline Variable & Keine & Mittel & Hoch & $\boldsymbol{p}$-Wert \\
\hline$\alpha$-Diversität: mittlere Artenzahl pro Subplot $\left(0.04 \mathrm{~m}^{2}\right)$ & $2,07 \pm 0,63^{\mathrm{a}}$ & $1,12 \pm 0,21^{\mathrm{b}}$ & $1,78 \pm 0,52^{\mathrm{a}}$ & $\mathbf{0 , 0 0 1}$ \\
$\gamma$-Diversität: kumulierte Artenzahl per Plot $\left(1000 \mathrm{~m}^{2}\right)$ & $35 \pm 7$ & $29 \pm 4$ & $29 \pm 3$ & 0,061 \\
$\beta$-Diversität (multiplikativ) & $17 \pm 4^{\mathrm{b}}$ & $27 \pm 5^{\mathrm{a}}$ & $17 \pm 5^{\mathrm{b}}$ & $<\mathbf{0 , 0 0 1}$ \\
Shannon-Evenness $\left(1000 \mathrm{~m}^{2}\right)$ & $0,32 \pm 0,13^{\mathrm{b}}$ & $0,55 \pm 0,12^{\mathrm{a}}$ & $0,39 \pm 0,18^{\mathrm{ab}}$ & $\mathbf{0 , 0 1 6}$ \\
Anzahl endemischer Arten $\left(1000 \mathrm{~m}^{2}\right)$ & $7 \pm 2$ & $7 \pm 1$ & $5 \pm 2$ & 0,096 \\
\hline
\end{tabular}

In Schmiedel et al. (im Druck) [25] analysieren wir die jährlichen Daten zu Individuenzahlen aller Gefäßpflanzenarten auf acht $25 \mathrm{~m}^{2}$ großen Plots in typischen Quarzfeld-Vegetationstypen der Knersvlakte über einen Zeitraum von 12 Jahren. Schon die gemeinsame Auftragung von Artenzahlen und Jahresniederschlägen macht visuell klar, dass Niederschläge die Artenzahl positiv beeinflussen (Abb. 26). So waren die Artenzahlen in den Jahren 1996 und 1997 signifikant über dem langjährigen Mittel und das waren genau auch die beiden Jahre mit den höchsten Niederschlägen unter allen Erfassungsjahren. Betrachtet man die Veränderungen etwas genauer (Abb. 27), sieht man dass das besonders feuchte Jahr zu besonders vielen (Re-) Etablierungen von Arten führte, während das besonders trockene Jahr 1998 die höchste lokale Aussterberate bei zugleich niedrigster (Re-) Etablierungsrate aufweist. Neben dem Artenreichtum haben wir in Schmiedel et al. (im Druck) [25] auch die Populationsdynamik aller Arten genauer analysiert, was hier nicht im Detail referiert werden soll. Einzig die auf Lebensformebene aggregierte Darstellung (Abb. 28) soll hier kurz angesprochen werden. Sie zeigt sehr schön - quasi lehrbuchhaft - wie die drei Lebensformen Therophyten, Geophyten und Chamaephyten unterschiedlich auf eine variable Umwelt reagieren. Therophyten reagieren extrem stark (die Darstellung von Abb. 
28 ist logarithmiert!) und extrem schnell auf überdurchschnittlichen Regen durch Abundanzzunahme und auf Trockenheit durch Abundanzrückgang, während die Reaktion der Chamaephyten stark gedämpft und verzögert ist und die Geophyten sich intermediär verhalten.
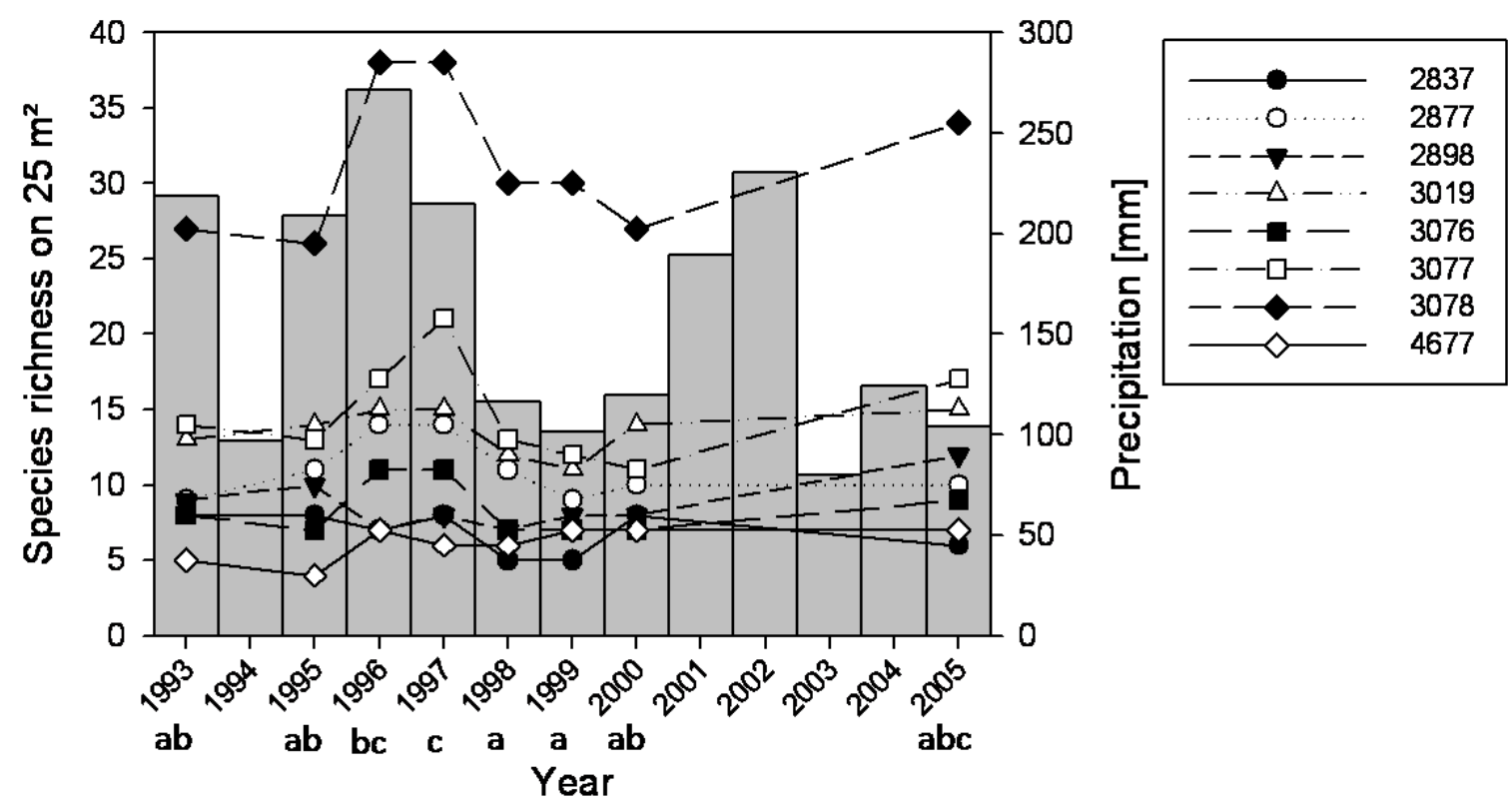

Abb. 26: Veränderungen der Artenzahlen der acht Quarzfeld-Dauerflächen in der Knersvlakte in Relation zum Jahresniederschlag der Station Vanrhynsdorp (aus Schmiedel et al. im Druck [25]). Die Buchstaben stehen für Unterschiede der jährlichen Artenzahl im Vergleich zum langjährigen Mittel, analysiert mit einer ANOVA und Tukeys HSD post-hoc-Test auf einem Signifikanzniveau von $\alpha=0,05$.

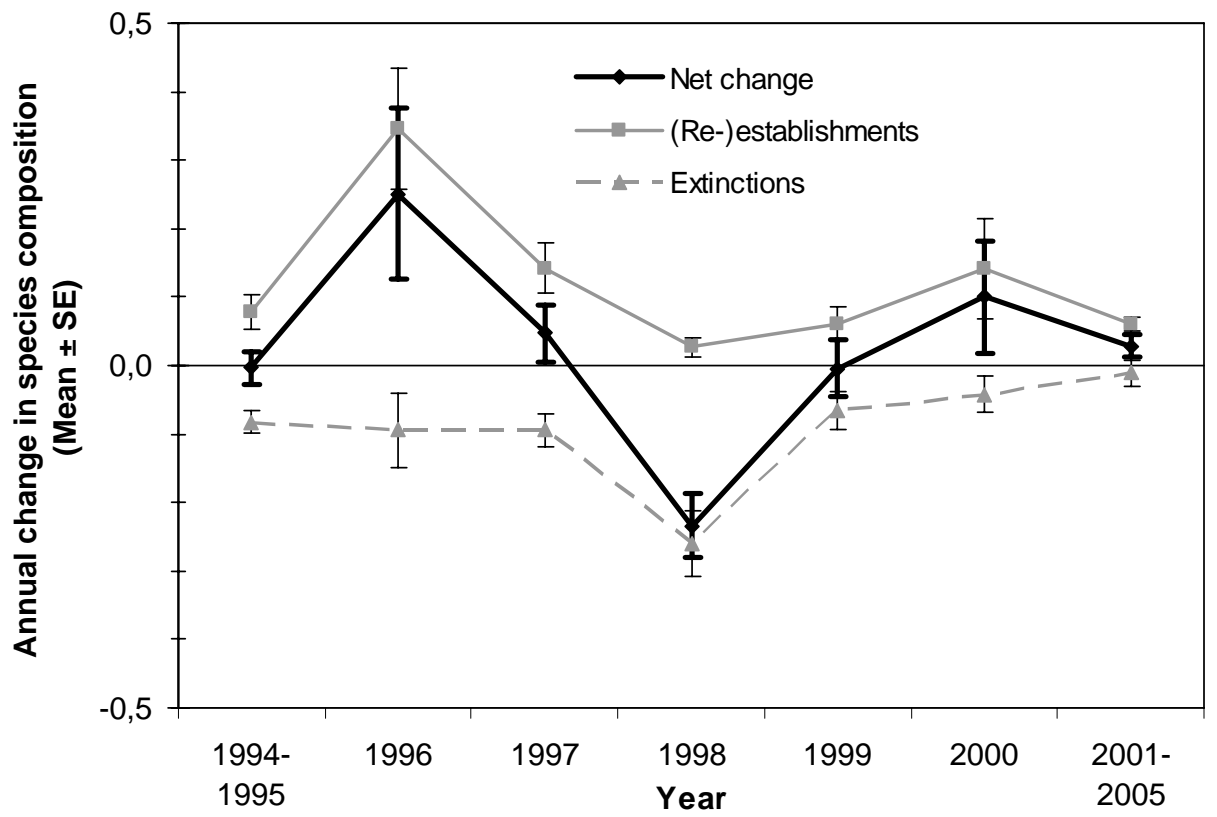

Abb. 27: Relative jährliche Änderungen in der Artenzusammensetzung (Mittelwert der acht Dauerflächen). Die Netto-Veränderungen sind aufgeschlüsselt in Gewinne und Verluste (aus Schmiedel et al. im Druck [25]). 


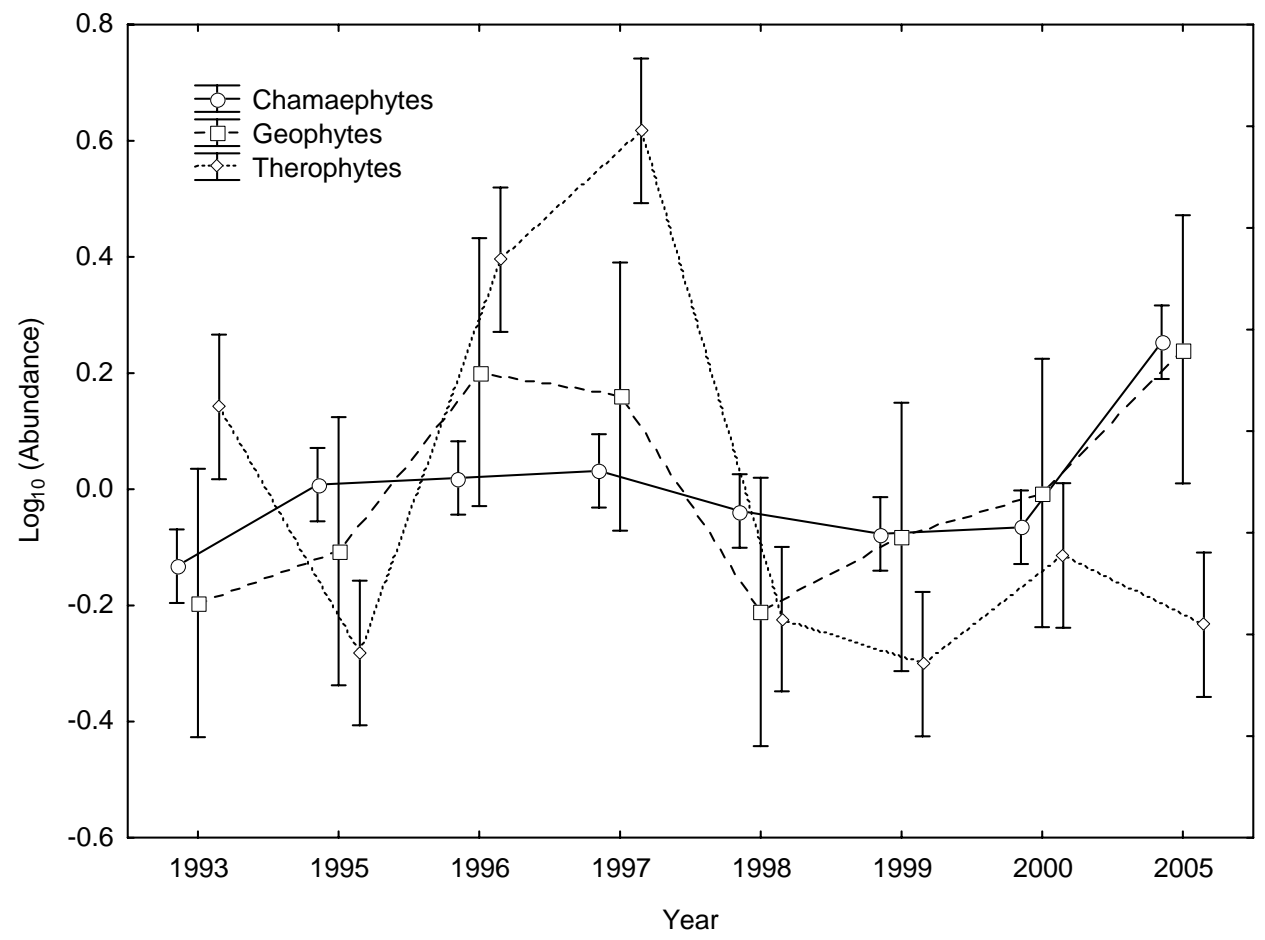

Abb. 28: Ergebnisse der zweifaktoriellen ANOVA der log-transformierten relativen Abundanzwerte in Abhängigkeit von Jahr und Lebensform. Sowohl das Jahr $\left(F_{7 ; 1216}=12,201\right)$ als auch die Interaktion Jahr $\times$ Lebensform $\left(F_{14 ; 1256}=13,949\right)$ waren hoch signifikant $(p<0.001)$, während die Lebensform alleine aufgrund der Definition der relativen Abundanz keinen Effekt hatte (aus Schmiedel et al. im Druck [25]). 


\section{Analyse und Diversität von Pflanzengesellschaften [26-38]}

\subsection{Methodik}

Pflanzensoziologie ist ein Teilbereich der Vegetationsökologie, der sich mit rezenten (vs. fossilen), taxonomischen (vs. physiognomischen oder funktionellen) Pflanzenvergesellschaftungen auf der räumlichen Skala von Beständen (vs. Landschaften oder Biomen) beschäftigt. Ihr grundlegendes Ziel ist die Definition und funktionelle Charakterisierung von Vegetationstypen basierend auf der vollständigen Artenkombination von Beständen [Übersetzung durch den Verfasser]. So haben wir in dem von mir initiierten Beitrag für die Encyclopedia of Ecology diese Disziplin der Ökologie definiert (Dengler et al. 2008 [26]). Der Pflanzensoziologie, also jener auf Josias Braun-Blanquet zurückgehenden, zunächst vor allem in Mittel- und Südeuropa vertretenen Schule der Vegetationsökologie (Braun-Blanquet 1964, Westhoff \& van der Maarel 1973, Dierßen 1990, Dierschke 1994) haftete lange etwas Verstaubtes an, zum Teil auch deshalb, weil manche Vertreter der Disziplin sich wenig offen für neue Methoden zeigten, die anderswo in der Vegetationsökologie entwickelt wurden. Wie Ewald (2003a) in seiner Critique for phytosociology herausstellt, war manche Kritik der Pflanzensoziologie oder an einzelnen ihrer exponierten Vertreter sicher gerechtfertigt, doch übersieht sie die großen Stärken der Pflanzensoziologie und ihren fundamentalen methodischen Beitrag zum Studium der Biodiversität auf der ökosystemaren Komplexitätsebene, im Fall der Pflanzen eben der Pflanzengesellschaften. (1) Durch das seit rund 100 Jahren weitgehend unverändert und millionenfach angewandte System der Vegetationsaufnahmen hat die pflanzensoziologische Schule der Vegetationskunde einen Datenfundus geschaffen, von dem Erdregionen ohne pflanzensoziologische Tradition nur träumen können (vgl. Abschnitt 2.2). (2) Die Pflanzensoziologie ist die einzige vegetationsökologische Schule die es bislang vermocht hat, ein konsistentes Klassifikationssystem kleinräumiger Vegetationstypen über große Räume zu schaffen, das erst eine Quantifizierung von und Kommunikation über botanische Diversität auf der Ebene von Vergesellschaftungen möglich macht und nicht auf banallaienhafter Ebene, etwa bei ökologisch kaum aussagekräftigen Einheiten wie „Buchenwald“ oder „Grasland“ stehen bleiben muss. Europas Pflanzensoziologen haben vor knapp einer Dekade erstmals eine Klassifikation der Vegetationstypen Europas bis hinunter zu den Verbänden vorgelegt (Rodwell et al. 2002) und eine wesentlich umfangreichere, aktualisierte Neuauflage befindet sich auch unter Beteiligung des Verfassers kurz vor der Fertigstellung (Mucina et al. in Vorb.). Diese detailliert floristisch abgegrenzten und ökologisch-chorologisch charakterisierten Vegetationseinheiten sind nicht nur essenziell für die Beschreibung der Untersuchungsobjekte in der Ökologie, ohne sie wäre auch eine so detaillierte Karte der natürlichen Vegetation wie sie Bohn 
et al. (2004) sie für ganz Europa erstellt haben, nicht denkbar gewesen, und auch die Habitattypen der Flora-Fauna-Habitat-Richtlinie (FFH-Richtlinie; englisch: Habitats Directive) der Europäischen Union, einem der wirksamsten Naturschutzinstrumente des Kontinentes, orientiert sich über große Strecken an pflanzensoziologischen Einheiten, da nur sie die notwendige ökologische Präzision haben (European Commission 2007). In den 1990er und 2000er Jahren begann dann quasi eine Renaissance der Pflanzensoziologie, v. a. getragen durch drei Entwicklungen, die sich gegenseitig befruchteten:

1. Entwicklung neuer Methoden zur Bestimmung diagnostischer Arten und zur Definition und Abgrenzung von Vegetationseinheiten, die sowohl weniger subjektiv als frühere Verfahren wie auch auf sehr große Datenbestände anwendbar sind (Bergmeier et al. 1990, Bruelheide 1995, 2000, Bruelheide \& Jandt 1997, Chytrý et al. 2002, Dengler 2003, Koči et al. 2003, Tichý 2005, Tichý \& Chytrý 2006, Willner 2006, 2011, Tsiripidis et al. 2009, Tichý et al. 2010).

2. Etablierung großer nationaler und regionaler Vegetationsdatenbanken (vgl. Dengler et al. $2011 \mathrm{~b}$ [4] und Jansen et al. 2011 [5])

3. Leistungsfähige und frei verfügbare Computerprogramme, die (1) und (2) ermöglichen, namentlich TURBOVEG (Hennekens \& Schaminée 2001) und JUICE (Tichý 2002).

In der Konsequenz entstanden einerseits in verschiedenen Regionen Europas erstmals konsistente und umfassende Klassifikationen sämtlicher Vegetationstypen größerer Gebiete basierend auf umfassenden Vegetationsdatenbanken (Großbritannien: Rodwell 1991 ff.; Niederlande: Schaminée et al. 1995 ff.; Slowakei: Valachovič 1995 ff. und Janišova 2007; MecklenburgVorpommern: Berg et al. 2001, 2004; Tschechische Republik: Chytrý 2007 ff.; Österreich: Willner \& Grabherr 2007), anderseits gewann die moderne Pflanzensoziologie in den letzten 15 Jahren weltweit auch in Gebieten Beachtung und Anwendung, in der sie zuvor nicht verbreitet war (siehe Dengler et al. 2008 [26]). Allerdings fehlte bislang ein modernes englischsprachiges Lehrbuch der Disziplin und die letzten veröffentlichten Lehrbücher sind auf Deutsch (Dierßen 1990, Dierschke 1994) und aus der Zeit vor der Renaissance der Pflanzensoziologie. Ziel von Dengler et al. (2008) [26] war es daher, eine kompakte Kurzeinführung in die Pflanzensoziologie für die internationale Leserschaft zu geben. Auf 13 Seiten bringen wir eine hoch kondensierten Überblick über Historie, Ziele, Begriffe, Konzepte, Methoden und Anwendungen der modernen Pflanzensoziologie, wie wir sie verstehen.

Eines der methodisch modernen, auf einer sehr großen Datenbank basierenden vegetationskundlichen Übersichtswerke ist Berg et al. (2001, 2004) für Mecklenburg-Vorpommern, an dessen Konzeption und Realisation ich maßgeblich beteiligt war. Nach Abschluss des Projektes 
haben wir unsere methodischen Vorschläge samt kritischer Reflektion und praktischen Erfahrungen bei ihrer Umsetzung in verschiedenen Publikationen für andere Anwender aufbereitet, von denen ich drei hier in die Habilitationsschrift einbeziehen möchte:

1. In Dengler et al. (2005) [27] stellen wir heraus, dass wir für moderne pflanzensoziologische Bearbeitungen größerer Gebiete drei Punkte als essenziell erachten: (a) konsistente Methodik für die Klassifikation aller real vorkommenden Vegetationstypen; (b) umfassende Dokumentation der verwendeten Methodik; (c) Präsentation der Ergebnisse in einer Weise, welche die Überprüfung der Methodenkonformität erlaubt. Wir stellen die Berg et al. (2001, 2004) zugrunde liegende, im Wesentlich auf Dengler (2003) beruhende Methodik vor, die sich in 12 Axiomen zusammenfassen lässt. Sie hat in Mecklenburg-Vorpommern eine de novoKlassifikation aller Vegetationstypen erlaubt und auf allen syntaxonomischen Ebenen zu klar abgegrenzten, ökologisch gut interpretierbaren Einheiten geführt, die in vielen Fällen mit zuvor publizierten Klassifikationskonzepten übereinstimmen, in einzelnen Bereichen aber auch neue Perspektiven aufzeigen. Ein wesentlicher Unterschied zu den meisten anderen aktuellen Ansätzen ist, dass das von Dengler et al. (2005) [27] präsentierte Verfahren in der Lage ist, alle real auftretenden Vegetationsbestände zu klassifizieren, insbesondere durch die umfassende Anwendung des Zentralsyntaxonkonzeptes (Dierschke 1981, 1994, Bergmeier et al. 1990 als „Verbandsbasalgesellschaft“, Dengler 2003). Dagegen führt die alleinige Fixierung auf positive Charakterisierung von Vegetationseinheiten (vgl. auch die Diskussion in Tsiripidis et al. 2009) in vielen anderen modernen Vegetationsklassifikationen dazu, dass ein erheblicher Teil der realen Vegetation nicht klassifiziert werden kann. So konnten für die Vegetation of the Czech Republic nur rund 30-50 \% aller vorhandenen Vegetationsaufnahmen mit Hilfe der verwendeten Artengruppenmethode (Bruelheide 2000, Koči et al. 2003) Assoziationen zugeordnet werden und ist entsprechend in den Vegetationstabellen wiedergegeben (Chytrý 2007). Das führt dazu, dass die Vegetationstabellen ein stark ,verzerrtes“ Bild der realen Vegetation geben; einige in der Tschechischen Republik sehr häufige Einheiten wie das überwiegend negativ charakterisierte Rubo caesii-Calamagrostietum epigeji fehlen in Konsequenz des gewählten Ansatzes sogar gänzlich (vgl. Chytrý 2009).

2. In Berg \& Dengler (2005) [28] stellen wir die große, bislang aber meist kaum gewürdigte und nur sehr unvollständig genutzte Bedeutung von Moos- und Flechtenarten bei der syntaxonomischen Klassifikation heraus. Der Blick auf diese Tatsache wurde in der Vergangenheit vielfach dadurch verstellt, dass etliche Bearbeiter Moose und Flechten in Vegetationsaufnahmen nicht notieren. Dadurch scheinen die Nicht-Gefäßpflanzenarten dann in Vegetationstabellen sehr viel seltener zu sein als sie tatsächlich sind, wenn Stetigkeiten wie häufig zu beobachten, 
methodisch aber falsch durch Division durch die Gesamtaufnahmenzahl statt nur durch die Zahl der Aufnahmen mit Moos- und Flechtenbearbeitung ermittelt wird. Wenn man diesen Fehler vermeidet, wie wir das in Berg et al. $(2001,2004)$ getan haben, zeigt sich, dass es sehr viel mehr diagnostische Arten unter diesen beiden Artengruppen gibt als die einschlägige pflanzensoziologische Literatur vermuten lässt. Moose kommen in allen Vegetationsklassen mit Ausnahme dreier stark salzbeeinflusster vor und spielen in den meisten auch eine Rolle als diagnostische Arten. Flechten sind zwar auf weniger Vegetationseinheiten beschränkt, haben dort aber dann eine umso größere Bedeutung als Charakter- und Differenzialarten.

3. Während auf der Ebene von Arten Rote Listen schon lange ein etabliertes und zentrales Instrument des Biodiversitätsschutzes sind und einer stetigen methodischen Weiterentwicklung unterliegen (Schnittler \& Ludwig 1996, Gruttke 2005, Ludwig et al. 2005, IUCN 2011), waren entsprechende Ansätze auf der ökosystemaren Ebene bislang wenig verbreitet. Seit den 1980er Jahre wurden in Deutschland zwar Rote Listen von Biotoptypen (BFANL 1986, Blab \& Riecken 1993) und seltener von Pflanzengesellschaften (Rennwald 2002; Übersicht bei Köppel 2002) erstellt, doch fehlte diesen meist eine den modern Roten Listen von Organismen vergleichbare stringente Methodik. In Timmermann et al. (2006) [29] präsentieren wir daher den von uns im Rahmen des Projektes „Pflanzengesellschaften MecklenburgVorpommerns und ihre Gefährdung“(Berg et al. 2001, 2004) entwickelten Ansatz. Bei dieser Bewertungsmethode standen drei Aspekte im Vordergrund: (a) klare Trennung von naturwissenschaftlicher Beschreibung und Prognose von der normativen Bewertung; (b) Anpassung der Kriterien einer Roten Liste an die Spezifika von Pflanzengesellschaften als Schutzobjekten; (c) eine möglichst objektive und transparente Gestaltung des Verfahrens. Wir differenzieren in eine naturwissenschaftliche Gefährdungseinstufung und eine normative „naturschutzfachliche Wertstufe“, die zusammen den Handlungsbedarf ergeben. Sowohl Gefährdungseinstufung als auch naturschutzfachliche Wertstufe setzen sich aus verschiedenen Einzelkriterien zusammen, die mittels Matrizen transparent und eindeutig aggregiert und schließlich zum Handlungsbedarf kombiniert werden. Die vorgeschlagene Methodik hat sich bei den Pflanzengesellschaften Mecklenburg-Vorpommerns hervorragend bewährt, enthält aber auch Aspekte die zur weiteren Optimierung der Bewertungsverfahren in Roten Listen anderer Schutzobjekte (Arten, Biotope) beitragen können.

\subsection{Trockenrasen im nordisch-baltischen Raum}

Die Daten zweier von mir betreuter Diplomarbeiten zu Trockenrasen auf Ostseeinseln Öland (Löbel 2002) bzw. Saaremaa (Boch 2005) boten die Grundlage zu einer umfassenden Revision 
der Trockenrasenvegetation der hemiborealen Zone Europas. Die ökologische Besonderheit, die hohe Diversität und die Großflächigkeit von Trockenrasengesellschaften Ölands hat schon seit Carl von Linnés erstem Besuch im Jahr 1741 die Botaniker und Ökologen fasziniert und war Anlass zu unzähligen Studien (z. B. Albertson 1950, Braun-Blanquet 1963, Krahulec et al. 1986, Sjögren 1988, van der Maarel 2008; vgl. Löbel \& Dengler 2008 [32]). Erst in jüngerer Zeit wurde klar, dass es im Westen Estlands, vor allem auf der Insel Saaremaa, ähnliche und ähnlich vielfältige Trockenrasentypen gibt (z. B. Pärtel et al. 1999), zu denen aus der Zeit vor dem Fall des Eisernen Vorhangs kaum Daten vorliegen. Ein besonderes und europaweit einmaliges Habitat der Ostseeinseln stellt die Vegetation über oberflächennah anstehenden Kalksteinplatten, die sogenannten Alvare, die als prioritärer Habitattyp 6280 (Nordic alvar and precambrian calcareous flatrocks) den besonderen Schutz der Europäischen Union genießen (European Commission 2007). Daneben gibt es in der Region aber auch vielfältige Trockenrasentypen auf Moränen, Sanderflächen und Küstendünen.

Die beiden Arbeiten von Dengler \& Löbel (2006) [30] und Dengler et al. (2006b) [31] beschäftigen sich mit den Gesellschaften der basiphilen Felsgrusgesellschaften (Alysso alyssoidisSedetalia) im nordisch-baltischen Raum und vergleichen diese mit entsprechenden Felsgrusgesellschaften in Mitteleuropa. Es zeigte sich, dass die nordisch-baltischen Gesellschaften zwar klar zur genannten Ordnung gehören, floristisch aber so verschieden von den aus dem temperaten Mitteleuropa beschriebenen Einheiten sind, dass sie nicht in den dortigen Verband Alysso alyssoidis-Sedion eingegliedert, sondern diesem als nordisch-baltisches Pendant gegenüber gestellt werden sollten (Abb. 29), das wir unter dem Namen Tortello tortuosae-Sedion albi Hallberg ex Dengler \& Löbel 2006 beschreiben, indem wir einen von Hallberg (1971) gemachten Vorschlag validieren. Innerhalb des nordischen Verbandes lassen sich insgesamt sechs Assoziationen differenzieren, die in zwei Gruppen zerfallen, einen Unterverband mit den Einheiten auBerhalb der Alvare (2.1 und 2.2 in Abb. 29) und einen Unterverband mit den streng auf die Alvargebiet beschränkten Einheiten (2.3-2.6 in Abb. 29). Die nordisch-baltischen Einheiten zeichnen sich durch sehr viel häufigeres Auftreten zahlreicher Moos- und Flechtensippen, einige arktisch-alpine Taxa. vermehrtes Auftreten mesophiler sowie einiger Staunässe bzw. zeitweilige Überflutung anzeigende Taxa aus, während therophytische Gefäßpflanzen in den mitteleuropäischen Einheiten häufiger sind. Dazu kommen einige junge Endemiten der Alvarflora. Insgesamt sind die nordisch-baltischen Einheiten, wenn man sie mit mitteleuropäischen Aufnahmen gleicher Flächengröße und mit sorgfältiger Kryptogamenbearbeitung vergleicht rund doppelt so artenreich, wobei der Unterschied bei Flechten und Moosen besonders ausgeprägt, doch auch bei den Gefäßpflanzen signifikant ist (Tab. 13). Mit unseren Untersuchungen unterstreichen wir, 
dass bei der Alvarvegetation nicht nur die bislang in der Literatur überwiegend untersuchten, stärker gefäßpflanzendominierten Typen bedeutsam sind, sondern dass gerade die ganz offenen, auf den ersten Blick vielleicht sogar „öde“ aussehenden Bestände extrem hohe Artendiversität einschließlich der Vorkommen von etlichen der insgesamt sehr wenigen Endemiten Nordeuropas (vgl. Jonsell \& Karlsson 2004) sowie auch eine große Diversität auf Gesellschaftsebene (mit sechs stark differenzierten Assoziationen) aufweisen.

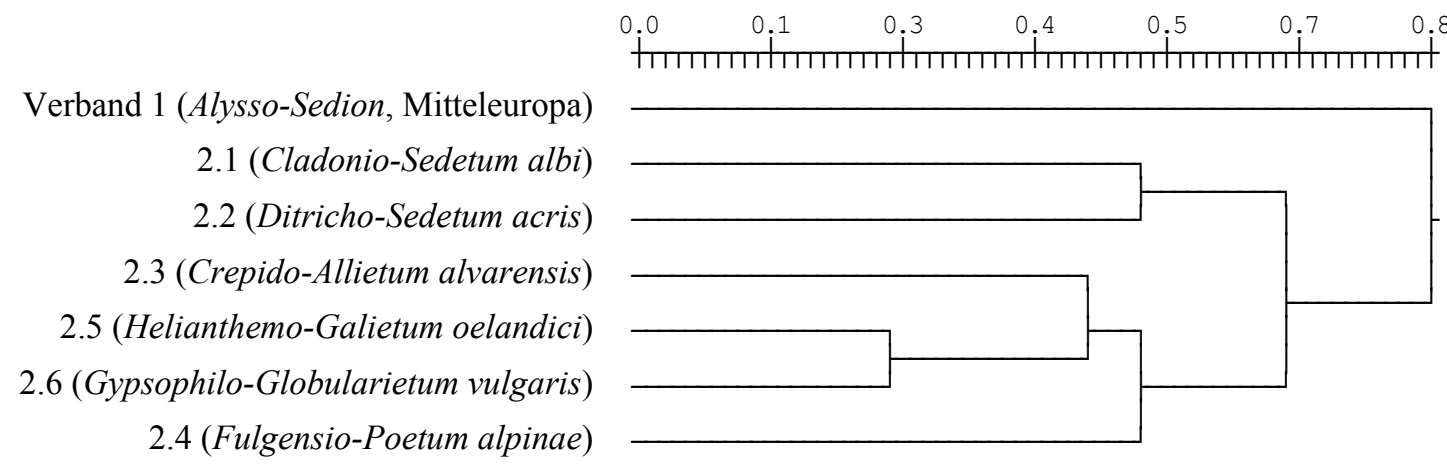

Abb. 29: Dendrogramm der nordischen Assoziationen (2.1-2.6) und des mitteleuropäischen Verbandes der AlyssoSedetalia. Die Clusteranalyse erfolge mit dem Bray-Curtis-Distanzmaß nach der Zuordnung der Aufnahmen zu den Syntaxa mittels der complete linkage-Methode (average linkage und single linkage erbrachten das gleiche Ergebnis) (verändert aus Dengler et al. 2006b [31]).

Tab. 13: Vergleich der Artendichten in verschiedenen Syntaxa der Alysso alyssoidis-Sedetalia auf $4 \mathrm{~m}^{2}$ (verändert aus Dengler \& Löbel (2006) [30]). Verband 1 ist das mitteleuropäische Alysso alyssoidis-Sedion, Verband 2 das nordisch-baltische Tortello tortuosae-Sedion albi mit seinen sechs Assoziationen (Namen siehe Tab. 13). Es sind jeweils die Mittelwerte \pm Standardabweichungen angegeben. Die hochgestellten Buchstaben bezeichnen homogene Gruppen gemäß Tukeys HSD post-hoc-Test auf einem Signifikanzniveau von $\alpha=0,05$.

\begin{tabular}{lccccccc}
\hline & Verband 1 & $\mathbf{2 . 1}$ & $\mathbf{2 . 2}$ & $\mathbf{2 . 3}$ & $\mathbf{2 . 4}$ & 2.5 & 2.6 \\
& $n=35$ & $n=6$ & $n=14$ & $n=74$ & $n=25$ & $n=41$ & $n=39$ \\
\hline Gesamtartenzahl & $17.7(6.9)^{\mathrm{a}}$ & $36.0(12.9)^{\mathrm{b}}$ & $41.4(9.7)^{\mathrm{b}}$ & $42.5(14.5)^{\mathrm{b}}$ & $48.4(12.0)^{\mathrm{bc}}$ & $52.9(10.8)^{\mathrm{c}}$ & $53.6(12.6)^{\mathrm{c}}$ \\
Gefäßpflanzen & $13.9(4.9)^{\mathrm{a}}$ & $23.3(4.6)^{\mathrm{bc}}$ & $22.3(4.6)^{\mathrm{bc}}$ & $19.2(8.2)^{\mathrm{b}}$ & $12.1(6.1)^{\mathrm{a}}$ & $20.5(6.2)^{\mathrm{bc}}$ & $23.2(4.3)^{\mathrm{c}}$ \\
Moose & $2.5(2.3)^{\mathrm{a}}$ & $7.2(6.4)^{\mathrm{b}}$ & $9.8(3.5)^{\mathrm{b}}$ & $17.7(7.8)^{\mathrm{c}}$ & $22.7(7.0)^{\mathrm{c}}$ & $19.3(7.6)^{\mathrm{c}}$ & $18.8(8.7)^{\mathrm{c}}$ \\
Flechten & $1.3(1.4)^{\mathrm{a}}$ & $5.3(2.9)^{\mathrm{b}}$ & $9.2(5.7)^{\mathrm{bc}}$ & $5.1(3.9)^{\mathrm{b}}$ & $13.0(4.6)^{\mathrm{d}}$ & $12.9(4.5)^{\mathrm{d}}$ & $11.5(5.2)^{\mathrm{cd}}$ \\
\hline
\end{tabular}

Boch \& Dengler (2006) [32] und Löbel \& Dengler (2008) [33] geben dann eine umfassende Beschreibung aller Trockenrasentypen der Inseln Saaremaa und Öland. Neben den schon besprochenen, besonders vielfältigen Felsgrusgesellschaften (Unterklasse Sedo-Scleranthenea der Koelerio-Corynephoretea) gibt es auch Sandtrockenrasen (Unterklasse Koelerio-Corynephorenea, Koelerio-Corynephoretea) und Kalktrockenrasen (Festuco-Brometea; siehe Abb. 30). Auch bei 
den Festuco-Brometea erwiesen sich die nordisch-baltischen Einheiten als so eigenständig, dass wir ihre Einordnung in einen eigenen Verband vorschlagen (Filipendulo vulgaris-Helictotrichion pratensis Dengler \& Löbel in Dengler et al. 2003), wohingegen die Sandtrockenrasenassoziationen durchweg weiter verbreiteten Einheiten entsprechen, wie sie etwa auch in Polen oder Nordostdeutschland vorkommen (z. B. Dengler 2004). Während sich die Artendichte in den verschiedenen Assoziationen auch deutlich unterscheidet (siehe Abb. 31), sind doch die Mittelwerte meist sehr hoch und die Maxima gehören zu den höchsten weltweit jemals auf dieser Skalenebene nachgewiesenen Werten (Öland: 80 Arten auf $4 \mathrm{~m}^{2}$; Saaremaa: 71 Arten auf $4 \mathrm{~m}^{2}$ ). Dabei ist der Anteil der Nicht-Gefäßpflanzen generell höher als in Trockenrasengesellschaften anderer Regionen und macht in allen Sedo-Scleranthenea-Gesellschaften im Mittel mehr als die Hälfte aller Arten aus (Löbel \& Dengler 2008 [33]).

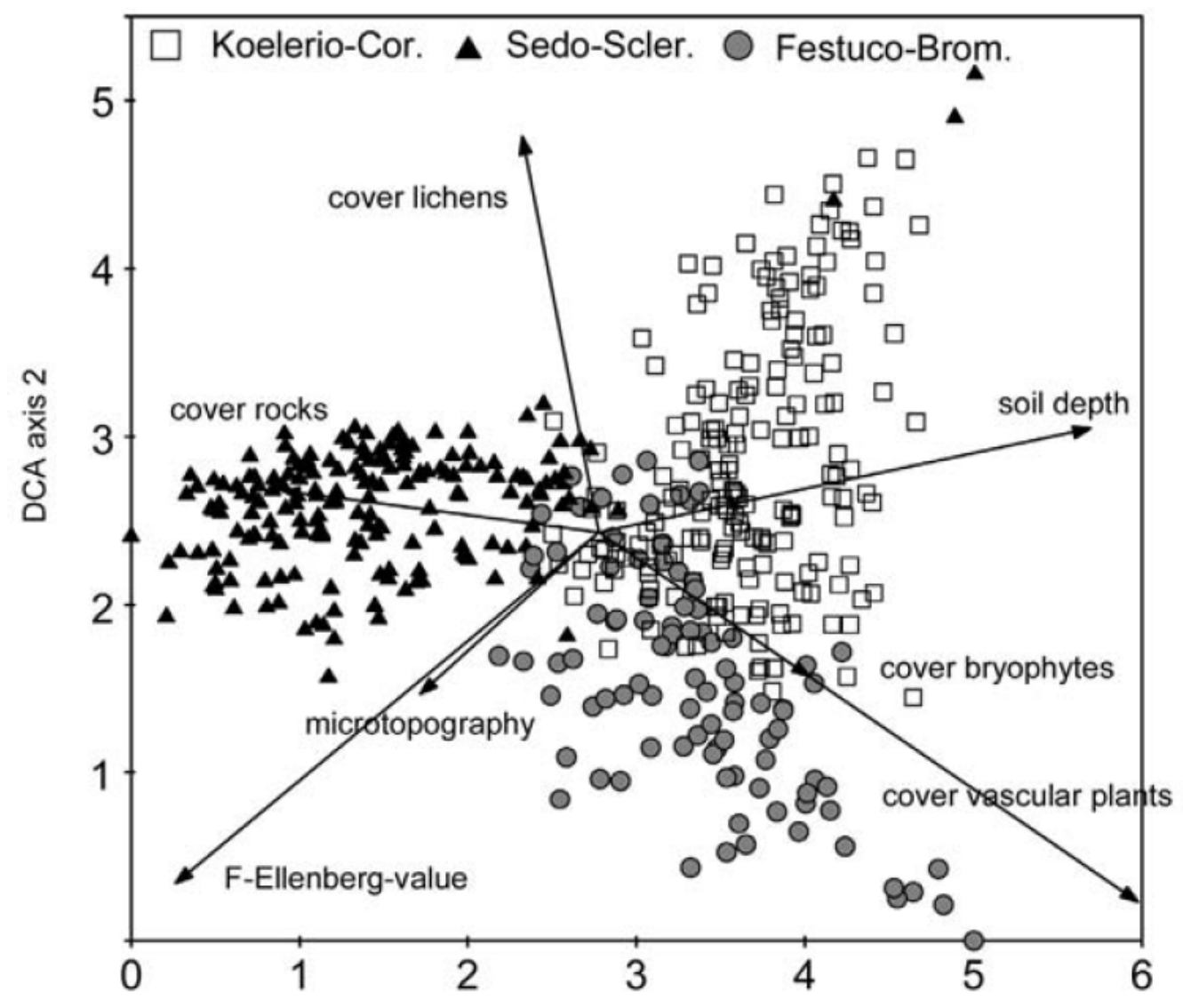

DCA axis 1

Abb. 30: Anordnung der 469 Vegetationsaufnahmen öländischer Trockenrasen (Lage der Aufnahmeflächen siehe Abb. 17) gemäß ihrer floristischen Ähnlichkeit in einem DCA-Ordinationsdiagramm (aus Löbel \& Dengler 2008 [33]). Die Symbole stehen für die drei vorkommenden hochrangigen Syntaxa (Quadrate: Koelerio-Corynephorenea; Dreiecke: Sedo-Scleranthenea; Kreise: Festuco-Brometea). Zu Interpretationszwecken wurden einige Struktur- und Umweltparameter nachträglich in das Ordinationsdiagramm geplottet. 


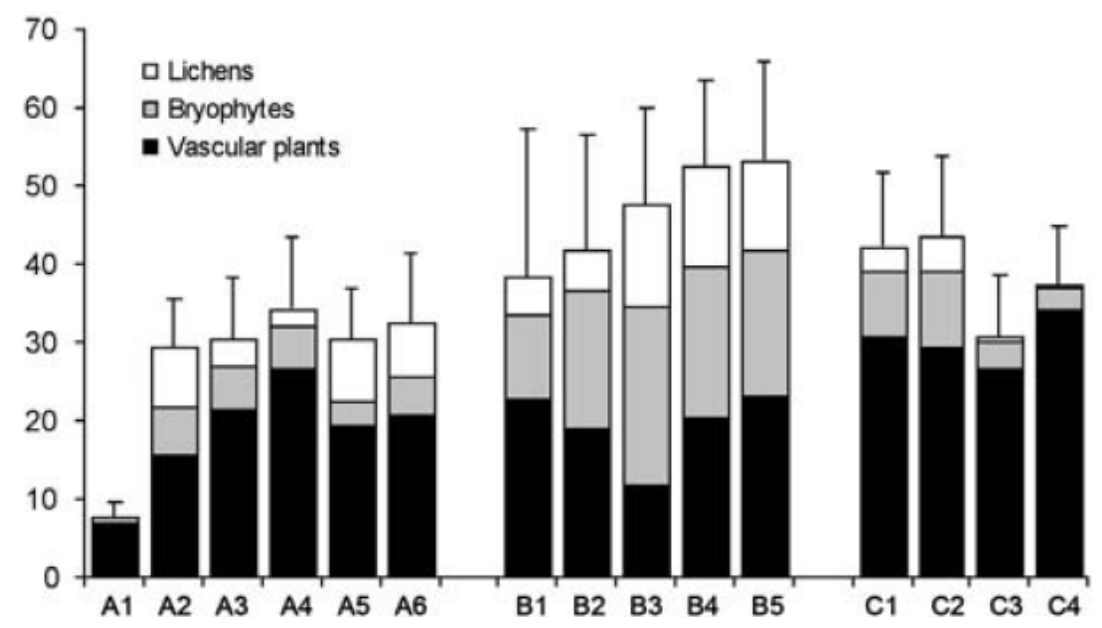

Abb. 31: Artendichte und Artengruppenzusammensetzung auf $4 \mathrm{~m}^{2}$ für die 15 auf Öland vorkommenden Trockenrasengesellschaften (aus Löbel \& Dengler 2008 [33]). Es sind Mittelwerte und Standardabweichungen dargestellt; schwarz $=$ Gefäßpflanzen; grau $=$ Moose $;$ wei $\beta=$ Flechten $; \mathrm{A}=$ Koelerio-Corynephorenea $; \mathrm{B}=$ Sedo-Scleranthenea $;$ $\mathrm{C}=$ Festuco-Brometea $)$.

Es ist in der Vergangenheit schon mehrfach hervorgehoben worden, dass nordische Pendants von schwerpunktmäßig im temperaten Europa verbreiteten Syntaxa nicht einfach die gleichen Einheiten oder eine „verarmte“ Entsprechung der mitteleuropäischen Einheiten darstellen (z. B. Braun-Blanquet 1963, Hallberg 1971, Dierschke 1974, Diekmann 1995, 1997). Doch nur in wenigen Fällen wurden aus solchen Befunden klassifikatorische Konsequenzen gezogen, meist blieb es - auch wegen des allgemein geringen Interesses nordeuropäischer Vegetationsökologen an Vegetationsklassifikation - bei der unbefriedigenden Gleichsetzung nordeuropäischer Einheiten mit ihren mitteleuropäischen Entsprechungen. In Dengler et al. (2006b) [31] beleuchten wir die Situation für fünf (Unter-) Klassen. Es zeigt sich, dass in fast allen Fällen die nordischen Bestände verglichen mit Mitteleuropa eine „ungewöhnliche“ Artenzusammensetzung haben (es kommen dort Arten gemeinsam vor, die dies in Mitteleuropa selten oder nie tun) und einen generell höheren Anteil von Moosen und Flechten (Tab. 14). Besonders stark abgesetzt mit extrem erhöhter Artendichte und Kryptogamenanteil sind die Sedo-Scleranthenea (s. o.), während die Koelerio-Corynephorenea des nordisch-baltischen Raums sich in den betrachteten Kriterien nur unwesentlich von jenen Mitteleuropas unterscheiden (Tab. 14). Besonders bemerkenswert ist, dass die kleinräumige Artendichte in drei der Syntaxa in der hemiborealen Zone deutlich höher ist als in der temperaten Zone, was die oft behauptete Universalität des latitudinalen Diversitätsgradienten vom Äquator zu den Polen in Frage stellt. Während auf größeren Skalenebenen (etwa $10.000 \mathrm{~km}^{2}$ wie bei Barthlott et al. 2005) Regionen in Mitteleuropa zweifelsohne eine höhere Phytodiversität verglichen mit solchen in Nordeuropa aufweisen, gilt dies im kleinskaligen 
Bereich offensichtlich nicht generell oder möglicherweise sogar generell nicht. Hier besteht noch Forschungsbedarf bezüglich der tatsächlich vorliegenden Muster und ihrer Ursachen.

Tab. 14: Vergleich der nordischen Gesellschaften von fünf (Unter-) Klassen mit ihren jeweiligen mitteleuropäischen Entsprechungen (modifiziert aus Dengler et al. 2006b [31]). $++=$ viel höher; $+=$ höher; $\pm=$ ähnlich; $-=$ niedriger; eingeklammerte Symbole stehen für geringfügige bzw. unsichere Unterschiede.

\begin{tabular}{lccccc}
\hline & $\begin{array}{c}\text { Sedo- } \\
\text { Scleranthenea }\end{array}$ & $\begin{array}{c}\text { Koelerio- } \\
\text { Corynephorenea }\end{array}$ & $\begin{array}{c}\text { Festuco- } \\
\text { Brometea }\end{array}$ & $\begin{array}{c}\text { Trifolio- } \\
\text { Geranietea }\end{array}$ & $\begin{array}{c}\text { Querco- } \\
\text { Fagetea }\end{array}$ \\
\hline "Ungewöhnliche" & $\mathrm{ja}$ & nein & $\mathrm{ja}$ & $\mathrm{ja}$ & $\mathrm{ja}$ \\
Artenzusammensetzung & & + & - & $(-)$ & - \\
Größe des Artenpools & + & + & $(+)$ & + & $?$ \\
Kleinräumige Artendichte & ++ & \pm & + & + & + \\
Anteil der Kryptogamen & ++ & $\mathrm{jein}$ & $\mathrm{ja}$ & $\mathrm{ja}$ & $\mathrm{ja}$ \\
Separate nordische Syntaxa & $\mathrm{ja}$ & & & &
\end{tabular}

\subsection{Saumgesellschaften Europas}

Basierend auf den Diplomarbeiten von Eisenberg (2003) und Krebs (2003) haben wir in Dengler et al. (2006a [34], 2007 [35]) die Saumgesellschaften Nordostniedersachsens umfassend analysiert und im europäischen Kontext betrachtet. Dabei wurde die Gesamtheit aller Saumgesellschaften grundwasserferner Standorte, und sowohl Außensäume als auch Innensäume analysiert. Eine Saumgesellschaft nach unserer Definition liegt vor, wenn sich Struktur- und Artenzusammensetzung deutlich sowohl von der angrenzenden Offenlandvegetation als auch von der Krautschicht der angrenzenden Gehölzvegetation unterscheiden (Abb. 32).

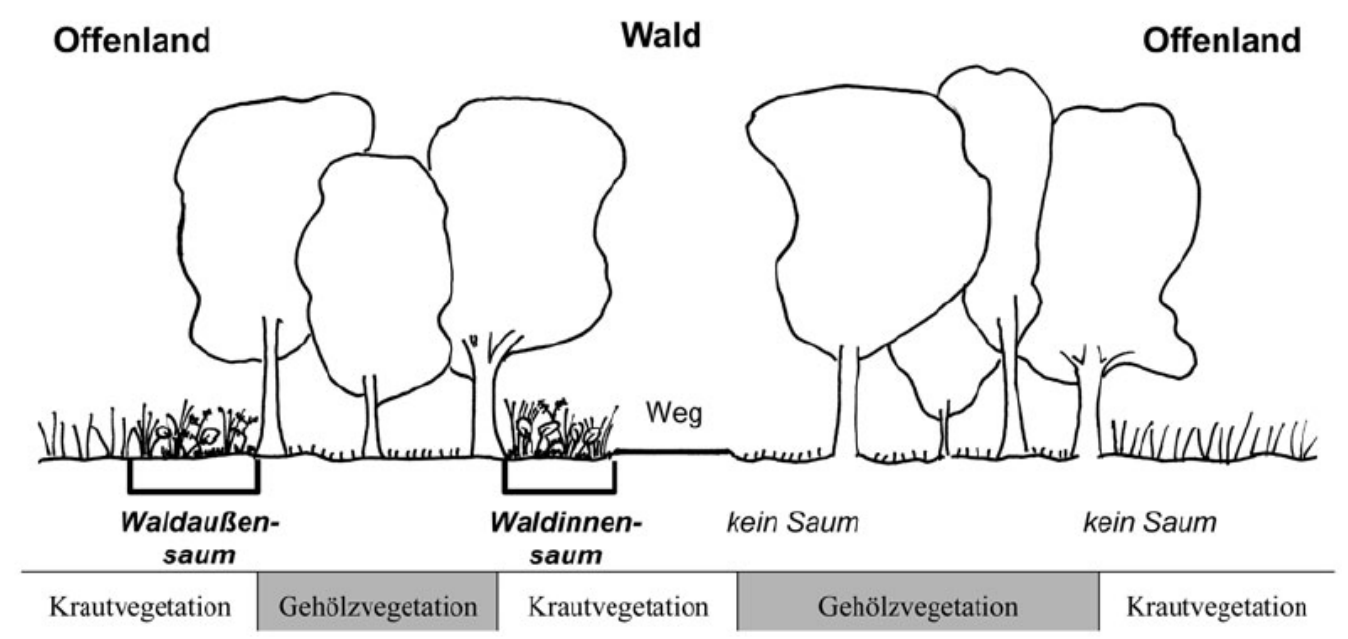

Abb. 32: Schematische Darstellung zur Erläuterung unserer strukturellen Saumdefinition sowie der Begriffe Waldaußensaum und Waldinnensaum (aus Dengler et al. 2006a [34]). 
Aufgrund der 446 eigenen Vegetationsaufnahmen aus Nordostniedersachsen und einer Analyse von Stetigkeitstabellen aus der Literatur aus großen Teilen Europas, die auf insgesamt mehr als 15.000 Vegetationsaufnahmen beruhen, schlagen wir eine syntaxonomische Klassifikation der Saumgesellschaften vor, die floristische und ökologische Beziehungen besser widerspiegelt als viele bisherige Gliederungsansätze. Die Hauptgliederung erfolgt anhand der Trophie in die nitrophytischen Säume der Klasse Artemisietea vulgaris und in die Säume magerer Standorte der Klasse Trifolio-Geranietea (Abb. 33, 33). Die in der Literatur fast immer vertretene Abgrenzung sogenannter „Schlagfluren“ (Klasse Epilobietea angustifolii) von den Artemisietea vulgaris und die oftmals vorgenommene Abtrennung azidophytischer Säume (Melampyro-Holcetea) von den Trifolio-Geranietea wurde weder durch unsere nordostdeutschen Aufnahmen noch durch die Analyse der Stetigkeitstabellen aus ganz Europa gestützt (Abb. 33). Dagegen konnten wir zeigen, dass es innerhalb der Trifolio-Geranietea zwei Gruppen von weit verbreiteten Assoziationen gibt, die bislang in syntaxonomischen Bearbeitungen weitgehend unberücksichtigt geblieben sind (Verbände Poion nemoralis und Violo-Stellarion holosteae). Wir konnten viele neue diagnostische Arten der Syntaxa herausarbeiten, insbesondere unter den Moosen, die von vielen früheren Saumbearbeitern gar nicht erhoben wurden, obwohl sie oft erhebliche Deckungen haben (Abb. 35).

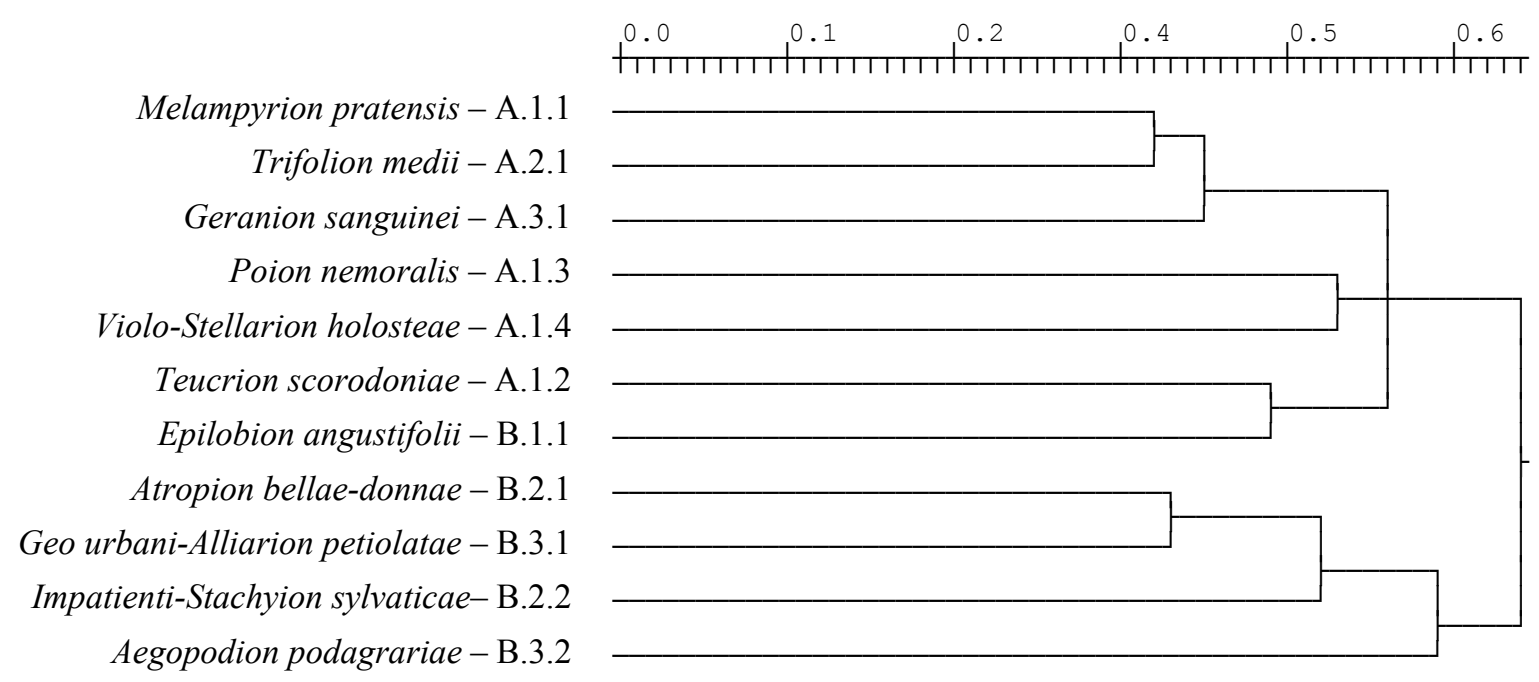

Abb. 33: Dendrogramm einer post hoc-Clusteranalyse für die 11 in Nordostniedersachsen vorkommenden SaumVerbände, berechnet auf Basis der Stetigkeitskennwerte. Die floristische Distanz wurde nach Bray-Curtis ermittelt und die Agglomeration fand aufgrund der Distanz zwischen den Gruppenzentroiden (average linkage) statt. Die Nummercodes geben die Hierarchie der Syntaxa wieder, die auf der Basis einer europaweiten Analyse von Stetigkeitstabellen unter Berücksichtigung des niedersächsischen Materials vorgeschlagen wurde. Man erkennt, dass die Clusteranalyse die Trennung der beiden Klassen (A: Trifolio-Geranietea; B: Artemisietea vulgaris) auch anhand des niedersächsischen Materials weitgehend bestätigt, die Anordnung der Verbände in Ordnungen dagegen überregional ein teilweise anderes Bild ergab als es der niedersächsische Datensatz nahelegte (verändert aus Dengler et al. 2006a [34]). 


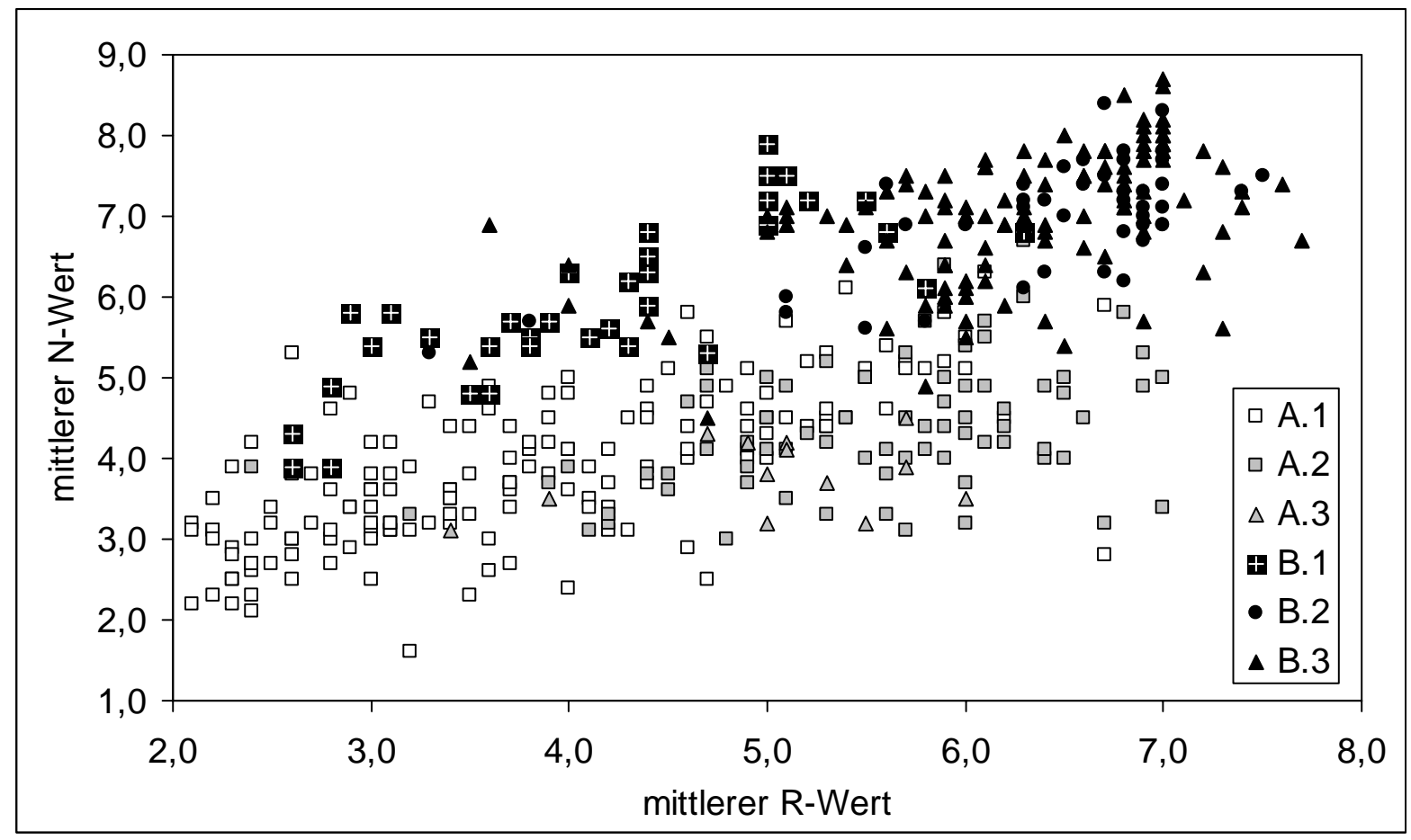

Abb. 34: Ökogramm der 446 Vegetationsaufnahmen von Saumgesellschaften aus Niedersachsen basierend auf mittleren Zeigerwerten für Reaktion $(\mathrm{R})$ und Stickstoff $(\mathrm{N})$. Die Symbole bezeichnen die sechs unterschiedenen Ordnungen: A.1 = Melampyro pratensis-Holcetalia mollis; A.2 = Origanetalia vulgaris; A.3 = Anthrisco ramosiGeranietalia sanguinei; B.1 = Galeopsio-Senecionetalia sylvatici; B.2 = Circaeo lutetianae-Stachyetalia sylvaticae; B.3 = Galio-Alliarietalia petiolatae (aus Dengler et al. 2007 [35]).

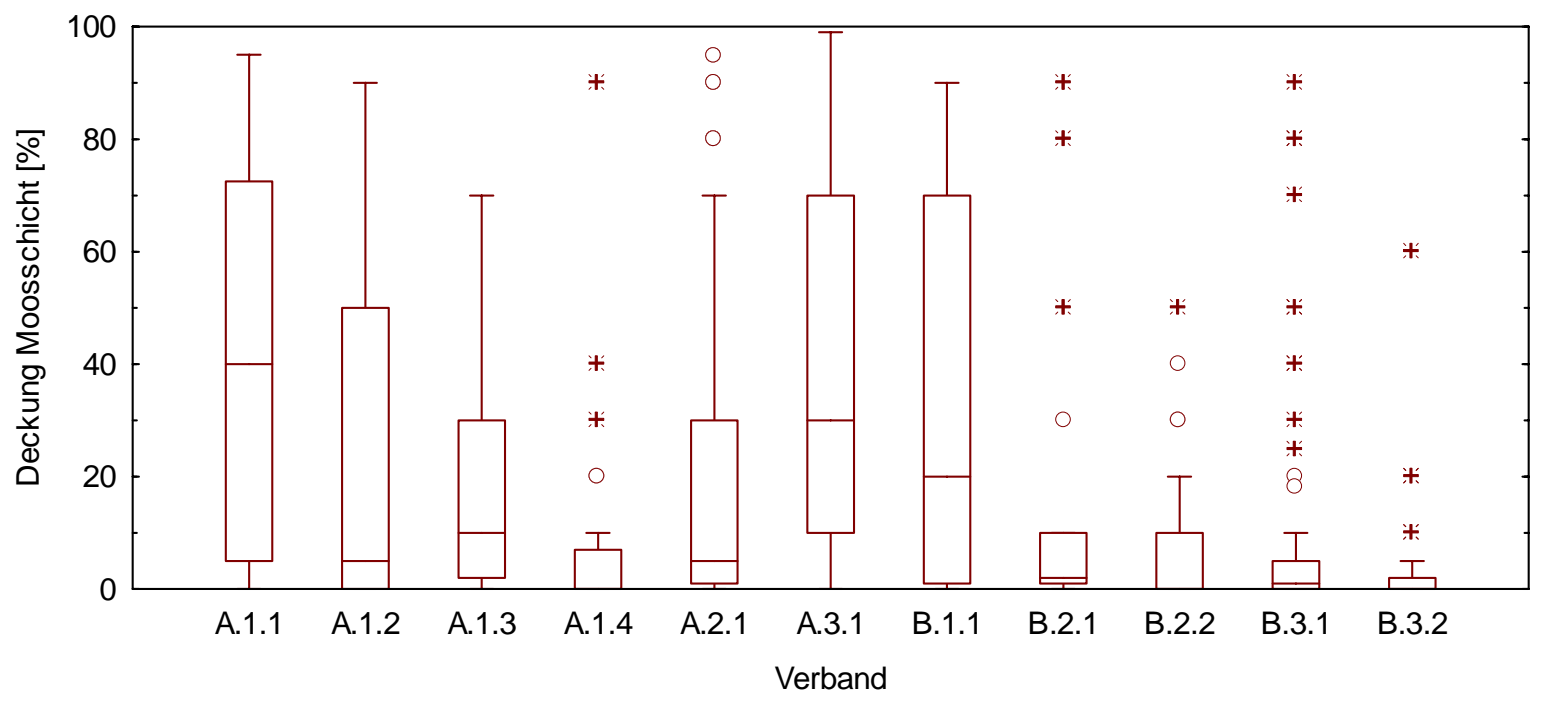

Abb. 35: Deckung der Moosschicht in Aufnahmen von niedersächsischen Saumgesellschaften (aus Dengler et al. 2007 [35]). Die Nummerncodes der Verbände sind in Abb. 33 aufgeschlüsselt. 
Die wesentlichen differenzierenden ökologischen Faktoren zwischen den hochrangigen Syntaxa illustriert Abb. 34 basierend auf Ellenberg-Zeigerwerten schön. Während der Nährstofffaktor die Klassen trennt, gibt es innerhalb jeder Klasse eine azidophytische Unterklasse (A.1, B.1) und eine basiphytische Unterklasse (A.2, A.3, B.2, B.3), was auch durch die direkte Analyse der Boden-pH-Werte bestätigt wurde (Dengler et al. 2007 [35]). Ein weiterer, bislang oft vernachlässigter Standortfaktor sind die Strahlungsverhältnisse, die wir mit einem Horizontoskop (Tonné 1954) ermittelt haben, und die vor allem auf Verbandsebene differenzieren (Abb. 36).

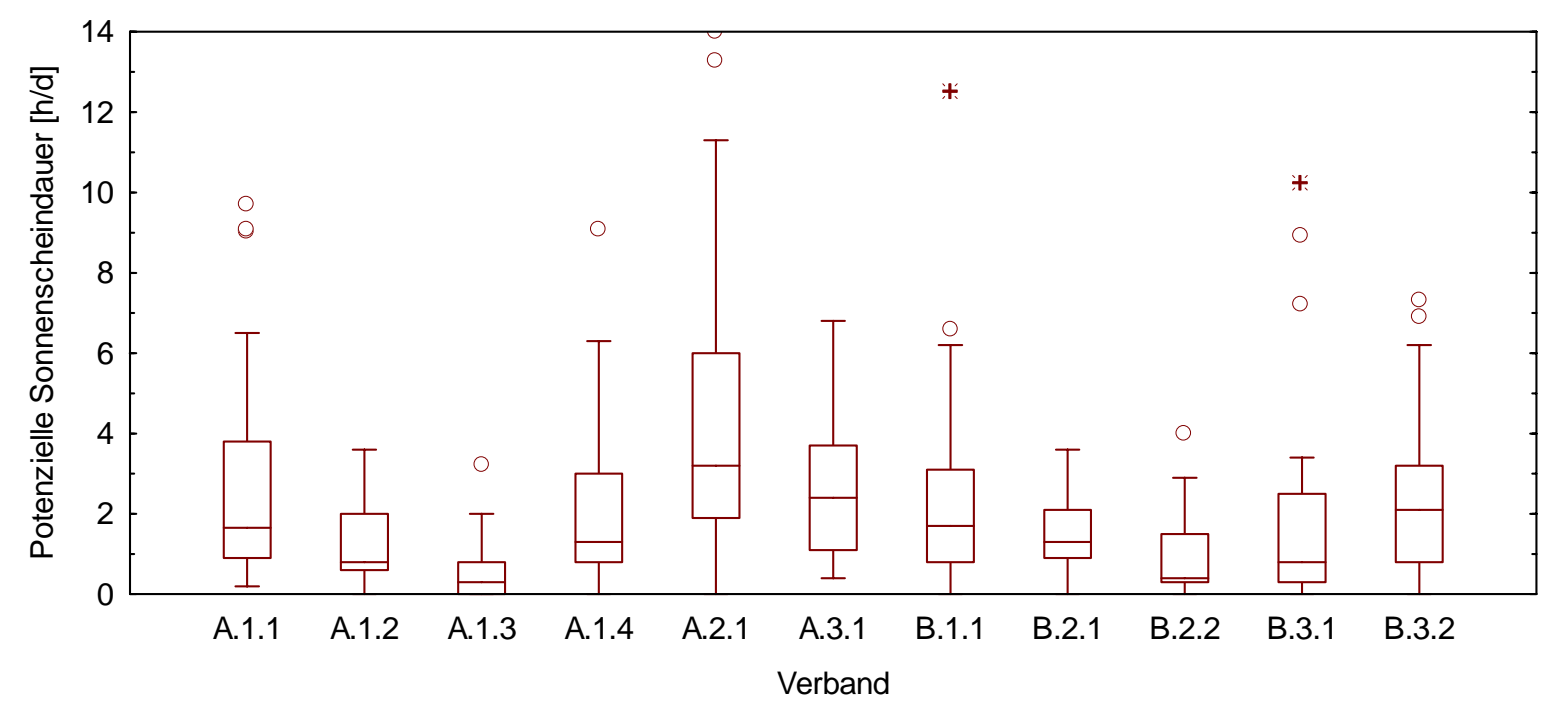

Abb. 36: Potenzielle mittlere Sonnenscheindauer während der Vegetationsperiode an den Standorten von niedersächsischen Saumgesellschaften (aus Dengler et al. 2007 [35]). Die Nummerncodes der Verbände sind in Abb. 33 aufgeschlüsselt.

Betrachtet man die Phytodiversität in den Saumgesellschaften, so zeigt sich, dass bezogen auf die $5 \mathrm{~m}^{2}$-Aufnahmeflächen die basiphytischen Säume magerer Standorte (A.2, A.3) gemeinsam mit den Circaeo-Stachyetalia (B.2) eine Gruppe bilden die eine signifikant höhere $\alpha$-Diversität aufweist als die Gruppe der anderen drei Ordnungen (azidophytische und nitrophytische Säume mit Ausnahme der Circaeo-Stachyetalia) (Dengler et al. 2007 [35]). Dies bestätigt das aus zahlreichen anderen temperaten und borealen Vegetationstypen bekannte Muster höherer Diversität in basenreichen Systemen (vgl. Abschnitt 4.3). Betrachtet man die kumulierte Diversität aller Saumaufnahmen auf den beiden untersuchten Messtischblättern 2728 (Lüneburg) und 2832 (Dannenberg [Elbe]), so ergibt sich ein erstaunliches Bild: Auf nur 0,0009 \% bzw. 0,0008 \% der Gesamtfläche des jeweiligen Messtischblattes fanden wir $34 \%$ bzw. $30 \%$ der insgesamt dort nachgewiesenen Gefäßpflanzenarten. Das zeigt zum einen die außerordentliche $\beta$-Diversität der 
Säume, die viel mit ihrem extremen Längen-Breiten-Verhältnis zu tun hat (vgl. Abschnitt 3.2), macht aber auch ihre Bedeutung für den Naturschutz deutlich.

Schließlich haben wir in einer kleinen Studie, die Saumgesellschaften der estnischen Insel Saaremaa auf nährstoffarmen Standorten (Trifolio-Geranietea) analysiert (Dengler \& Boch 2008a [36]). Diese sind dort sehr großflächig und blütenbunt ausgebildet, waren aber nie zuvor dokumentiert worden. Wir konnten die Vielfalt der dort vorkommenden Saumgesellschaften 12 Gesellschaften in fünf Verbänden und drei Ordnungen zuordnen, wobei aufgrund des vorherrschenden basenreichen Untergrundes die azidophytische Ordnung Melampyro-Holcetalia nur mit einer einzigen und nicht besonders typisch ausgeprägten Assoziation vorkam. Die estnischen Säume sind mit16 bis 48 Pflanzenarten auf $5 \mathrm{~m}^{2}$ besonders artenreich. Dies konnten wir im Vergleich zu den selbst erhobenen Aufnahmen aus Niedersachsen (Dengler et al. 2006a [34]: gleiche Methodik) und Nordostdeutschland (unpubl.; $10 \mathrm{~m}^{2}$, mittels SAR auf $5 \mathrm{~m}^{2}$ umgerechnet) zeigen. Demnach waren alle Assoziationen, die sowohl in Estland als auch in einer der norddeutschen Regionen vorkamen in Estland meist signifikant artenreicher, im Vergleich zu Niedersachsen ( $n$ =7) um den Faktor 1,4-2,2, im Vergleich zu Nordostdeutschland ( $n=6)$ um den Faktor 1,2-1,9. Dies ist ein weiteres Beispiel, wo der latitudinale Diversitätsabfall vom Äquator zu den Polen im kleinskaligen Bereich nicht gilt, obwohl zumindest in Nordostdeutschland auch der Artenpool an Saumarten höher ist als in Estland. Wie in anderen Vegetationstypen im Norden (vgl. Abschnitt 5.2), beobachtet man auch in den Säumen, dass hier Arten zusammen vorkommen, die dies in Mitteleuropa normalerweise nicht tun würden, z. B. ausgesprochene Basiphyten gemeinsam mit ausgesprochenen Azidophyten (vgl. Abschnitt 5.2). Die spannende Frage ist das Warum. Mögliche Erklärungen könnten eine geringere Konkurrenzkraft von Arten bei kürzerer Vegetationsperiode oder geringerer atmogener Nährstoffeintrag sein, was beides höheren kleinräumigen Artenreichtum begünstigen könnte (Dengler \& Boch 2008a [36]). Das sind aber bislang nicht viel mehr als Spekulationen und die Erklärung dieses unerwarteten, aber wissenschaftlich umso spannenderen Befundes bedarf weiterer empirischer Beobachtungen (gilt das auch für weitere Vegetationstypen? gilt das auch im altitudinalen Gradienten? überlappen die Nischen von Pflanzenarten in der hemiborealen Zone mehr als in der temperaten?) und dann ggf. auch Experimente.

\subsection{Montan-subalpine Hochstaudengesellschaften Europas}

Die montan-subalpine Hochstaudengesellschaften der Mulgedio-Aconitetea sind in den Mittelund Hochgebirgen Europas eine allgemein verbreitete Vegetationsklasse. Wie für die meisten anderen Vegetationsklassen auch gab es datenbankgestützte moderne Klassifikationen bislang 
nur auf regionaler Ebene (Slowakei: Kliment et al. 2007, Tschechische Republik: Koči 2007). In der Arbeit von Michl et al. (2010) [37] haben wir die vom Erstautor erstellte umfassende Datenbank der Klasse genutzt, um zumindest für Mittel- und Nordeuropa einer konsistenten großskaligen Klassifikation näherzukommen. Wir waren hierbei mit vielen Problemen konfrontiert, die alle solchen supranationalen oder gar europaweiten Klassifikationen, von denen aufgrund der zunehmenden Verfügbarkeit nationaler Vegetationsdatenbanken (vgl. Abschnitt 2.2) in den nächsten Jahren mutmaßlich zahlreiche entstehen werden, Probleme bereiten. Insofern leistet unsere Studie auch ein Stück „Pionierarbeit“. Einige wichtige solche Probleme und unsere Lösungsvorschläge sind:

1. Während die gemeinsame Klassifikation von Vegetationsaufnahmen unterschiedlicher Flächengrößen die Ergebnisse massiv verfälschen kann (siehe Abschnitt 3.5), sind in der pflanzensoziologischen Literatur Europas sehr unterschiedliche Flächengrößen im Gebrauch. Während man optimalerweise nur eine einzige Flächengröße verwenden sollte, lässt sich das bei Literaturdaten nicht realisieren. Wir haben deshalb geschaut für welche ungefähr fünffachen Plotgrößen-Bereich, der von Dengler et al. (2009) [13] als gerade noch vertretbar angesehen wird, die größte Aufnahmezahl und die beste geografische und syntaxonomische Abdeckung vorliegt. In unserem Fall haben wir uns für den Bereich 9-50 $\mathrm{m}^{2}$ entschieden.

2. Da man europaweit anders als in den Ländern mit umfassenden nationalen Datenbanken nicht sämtliche Vegetationstypen auf einmal klassifizieren kann, wird man auf absehbare Zeit immer die Aufnahmen des zu betrachtenden Vegetationstyps (in unserem Fall also der Mulgedio-Aconitetea) in einer a priori-Selektion aus den einzelnen Datenbanken extrahieren müssen. Dies ist ein für das spätere Ergebnis entscheidender Schritt, doch sind die beiden hier bislang meist verfolgten Ansätze sehr problematisch. (a) Verwendet man die ursprüngliche $\mathrm{Zu}$ ordnung der Autoren der Aufnahmen zu einem höheren Syntaxon, wird man kaum die inkonsistente Sicht unterschiedlicher Bearbeiter aus vielen Ländern überwinden können; zudem gibt es viele Aufnahmen in Datenbanken, die gar nicht klassifiziert wurden. (b) In jüngerer Zeit wurden für solche supranationalen Studien manchmal a priori-Selektionen anhand der Deckungsgrade weniger dominanter Arten vorgenommen (z. B. Illyés et al. 2007). Dieser Ansatz ist zwar einfach zu implementieren und lässt sich hervorragend reproduzieren, hat aber den Nachteil, dass man im ausgewählten Aufnahmekollektiv viele eigentlich nicht in das Syntaxon gehörende Aufnahmen hat, während anderseits viele, die hineingehören würden, fehlen. Wir haben daher, einen Vorschlag von Dengler et al. (2006c) aufgreifend, die a prioriSelektion mittels a priori-Kennartenlisten für die gewünschte Klasse und für alle Nachbarklassen vorgenommen. Jede Aufnahme in der Datenbank wurde dann derjenigen Klasse zu- 
geordneten, deren diagnostische Arten (deckungsgewichtet) dominierten. Ähnlich wie Methode (b) ist dieses Verfahren exakt reproduzier- und übertragbar, kommt aber den üblichen Syntaxonabgrenzungen sehr viel näher, die sich ja auch an der gesamten Artenkombination und nicht an wenigen dominanten Arten festmachen. Ein wesentlicher Aspekt ist, dass hier eine Abwägung zwischen verschiedenen Klassen stattfindet. Zwar ist auch hier eine subjektive a priori-Festlegung der Kriterien (hier der Arten) nötig, aber in Fällen, in denen die Meinungen über den Kennwert einer Art in der Literatur auseinander gehen, kann man sie entweder unberücksichtigt lassen oder beiden Syntaxa zuweisen. Da in jedem Fall sehr viel mehr Arten zur Beurteilung herangezogen werden als bei Methode (b), ist die Vorauswahl wenig sensitiv gegenüber der a priori-Beurteilung einzelner Arten.

3. Gerade bei überregionalen Klassifikationen erlangt die adäquate klassifikatorische Behandlung negativ gekennzeichneter Einheiten große Bedeutung (vgl. Abschnitt 5.1).

Basierend auf diesen und einigen weiteren Überlegungen haben wir ein Startdatenset von 993 Aufnahmen aus dem gesamten temperaten Europa (Abb. 37) und aus Skandinavien erhalten. Dieses haben wir zunächst einer Clusteranalyse unterzogen, die sechs Cluster erbrachte, von denen fünf weitgehend Verbänden in herkömmlichen Gliederungen entsprachen, während der sechste alle skandinavischen Aufnahmen vereinte (Abb. 38). Diese waren in bisherigen Gliederungsvorschlägen immer unter den aus Mitteleuropa beschriebenen Verbänden subsummiert worden (Dierßen 1996, Rodwell et al. 2002). Bei einer Analyse möglicher diagnostischer Arten für die die sechs Verbände und mögliche übergeordnete Einheiten (Ordnungen), zeigte sich, dass die vom Dendrogramm suggerierte Teilung in zwei Gruppen mit je drei Verbänden nicht gut belegt ist, während eine Zusammenfassung der mitteleuropäischen fünf Verbände in einer Ordnung und ihrer Gegenüberstellung zu einer neuen skandinavischen Ordnung durch eine große Zahl von Kenn- und Trennarten gestützt wird. Entsprechend haben wir diese Lösung auch umgesetzt. Es zeigte sich hier wieder einmal, dass sich eine Clusteranalyse normalerweise nicht $1: 1$ in eine sinnvolle syntaxonomische Gliederung umsetzen lässt, sondern einer „Nachbearbeitung“ bedarf, wenn das Ziel floristisch gut charakterisierte Einheiten sind. Im vorliegenden Fall lag das Problem vermutlich daran, dass wir relativ wenige Vegetationsaufnahen aus Skandinavien zur Verfügung hatten, während viele numerische Verfahren sensibel auf „unbalancierte“ Datensets reagieren. Die Kriterien der Nachbearbeitung müssen allerdings transparent gemacht werden. Weiterhin zeigte sich einmal mehr, dass die Eigenständigkeit vieler nordischer Vegetationstypen bislang verkannt wurde, weil einerseits die Skandinavier wenig eigenes Interesse daran hatten, ihre Vegetation zu klassifizieren, andererseits Mitteleuropäer, die in Skandinavien vegetati- 
onskundlich arbeiteten, oft dazu neigten, die dortigen Vegetationstypen unter ähnlichen Einheiten zu subsummieren, die sie aus ihrer Heimat bereits kannten.

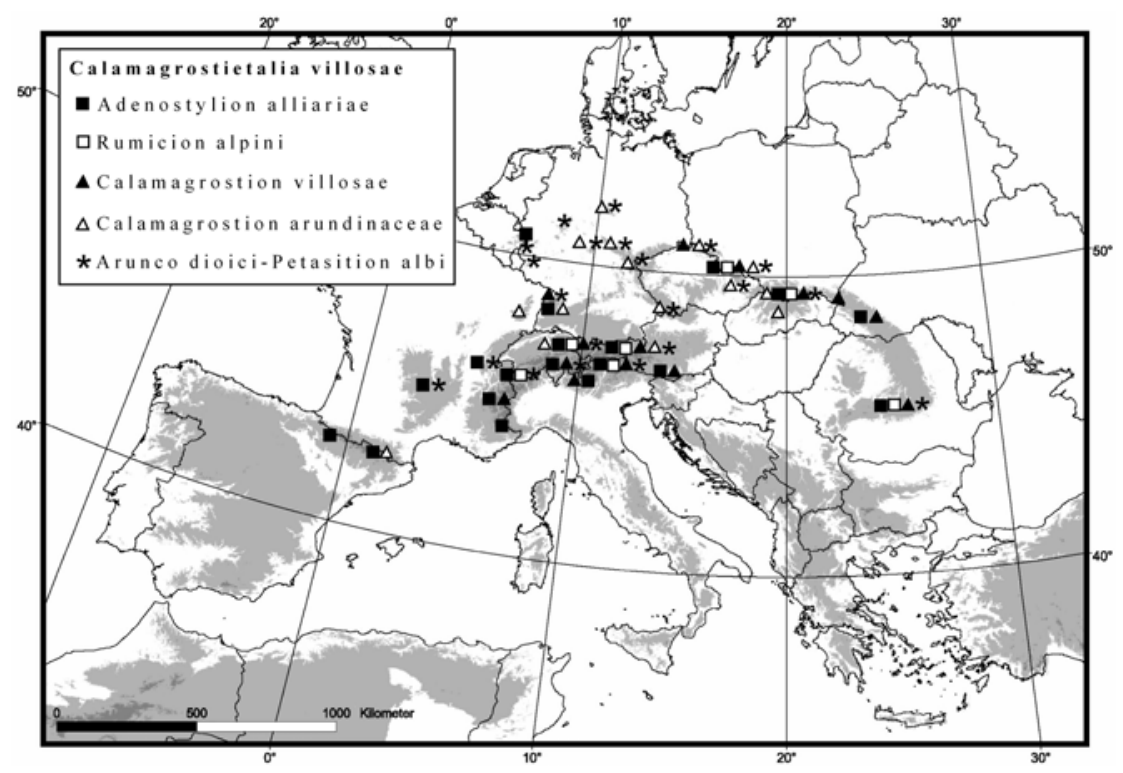

Abb. 37: Herkunft der Aufnahmen der Mulgedio-Aconietea-Aufnahmen aus dem temperaten Europa und ihre Zugehörigkeit zu den unterschiedenen fünf Verbänden (aus Michl et al. 2010 [37]).

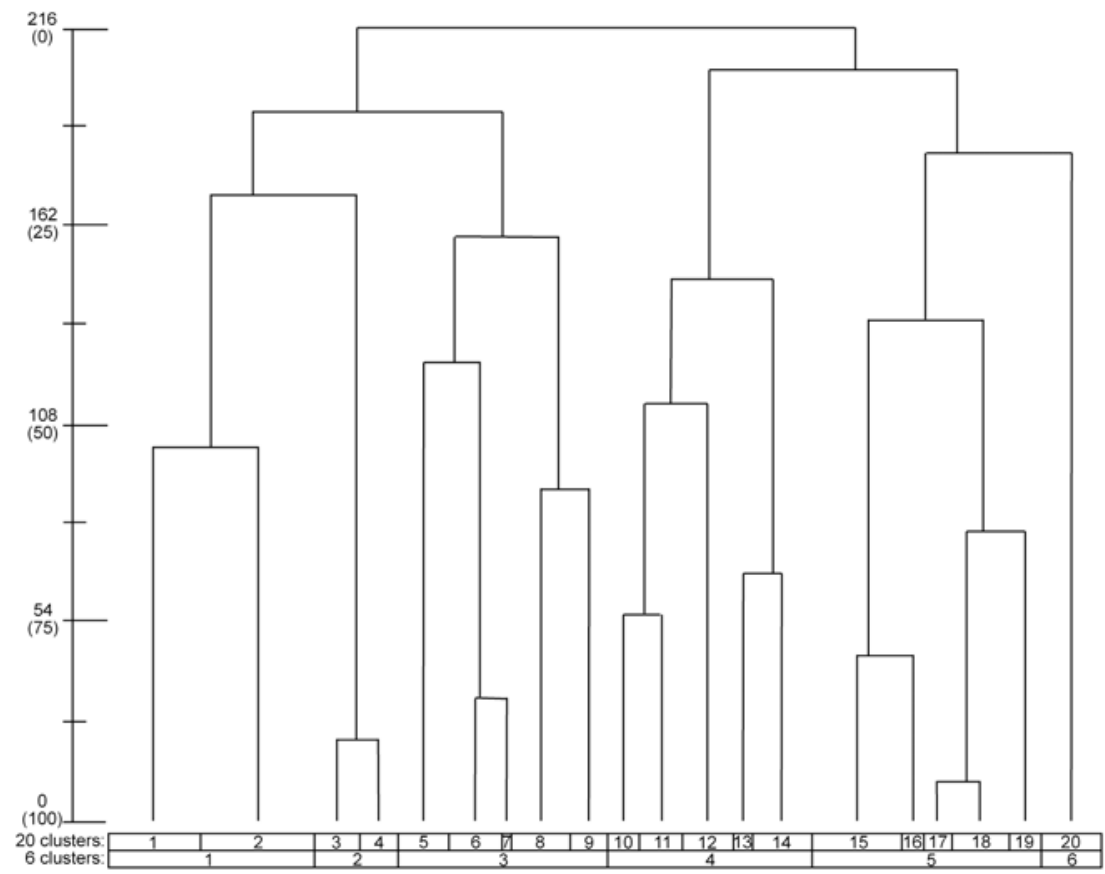

Abb. 38: Ergebnis der Clusteranalyse der 993 Aufnahmen europäischer Mulgedio-Aconitetea-Gesellschaften. Dargestellt sind die 6-Cluster-Lösung und die 20-Cluster-Lösung die als Verbände bzw. als Assoziationen interpretiert wurden. Bei der 6-Cluster-Lösung bedeuten: 1 = Adenostylion alliariae, 2 = Rumicion alpini, 3 = Calamagrostion villosae, 4 = Calamagrostion arundinaceae, 5 = Arunco dioici-Petasition albi, $6=$ Epilobio lactiflori-Geranietalia sylvatici (aus Michl et al. 2010 [37]). 


\subsection{Vegetation der Sukkulenten Karoo im südlichen Afrika}

In Südafrika gibt es seit Kurzem eine landesweite Vegetationskarte (Mucina \& Rutherford 2006), die bezogen auf den ganzen afrikanischen Kontinent ihresgleichen sucht. Ihre Kartiereinheiten entsprechen dabei Einheiten der potenziell natürlichen Vegetation, wie sie in Europa gebräuchlich sind (Bohn et al. 2004). Sie umfassen auch auf unterster Ebene fast immer Komplexe verschiedener Vegetationstypen, die beim vorgegebenen Kartiermaßstab nicht auflösbar waren (Mucina et al. 2006). Für viele Fragen der Grundlagenforschung und der angewandten Forschung (z. B. Weidemanagement, Naturschutz) bedarf es aber Kartiereinheiten, die sowohl die oft ausgeprägten kleinräumigen edaphischen Unterschiede auflösen, die etwa für die Vegetation der Sukkulenten Karoo prägend sind, als auch eine Differenzierung von Vegetationstypen erlaubt, die bei zunehmender Nutzungsintensität die natürliche Vegetation ersetzen. Während in der südafrikanischen Literatur durchaus regelmäßig lokale und regionale Studien basierend auf Vegetationsaufnahmen publiziert werden, begnügen sich diese doch fast immer mit formlosen Beschreibungen der gefundenen Einheiten, wodurch sich die Autoren in der Regel auch aus der Notwendigkeit entlassen fühlen, ihre Ergebnisse in einen überregionalen Kontext einzuordnen und zu diskutieren. In der Sukkulenten Karoo fehlten selbst solche Fallstudien weitgehend, obwohl dieses Biom einer der Biodiversitäts-Hotspots weltweit ist (Myers et al. 2000).

Die Arbeit von Luther-Mosebach et al. (im Druck) [38] hat insofern Pilotcharakter, als wir darin basierend auf 355 Vegetationsaufnahmen eine pflanzensoziologische Regionalbearbeitung vornehmen (Abb. 39), die aktuellen europäischen Maßstäben entspricht (z. B. Dengler et al. 2008 [26]), wie sie bislang in Südafrika nicht üblich waren. Die Vegetationsaufnahmen von einheitlich $100 \mathrm{~m}^{2}$ Größe (zur Bedeutung dieses Aspektes vgl. Abschnitt 3.5) wurden überwiegend nach einem randomisiert-zufälligen Verfahren ausgewählt und an ihren Standorten zahlreiche Relief-, Boden-, Struktur- und Nutzungsparameter ermittelt.

Wir konnten im Gebiet zwei pflanzensoziologische Klassen differenzieren und formal beschreiben, die sich in vier Ordnungen mit sechs Verbänden und 16 Assoziationen gliedern. Die Hauptdifferenzierung folgt dabei dem größten floristischen und ökologischen Gradienten zwischen der Klasse Hermannio trifurcae-Zygophylletea morgsanae der Matrixvegetation auf salzarmen Böden und der Klasse Didelto carnosae-Cephalophylletea inaequalis auf Salzböden (Tab. 15). Eine weitere Klasse dürften die stark differenzierten Vegetationstypen der Quarzfelder sein (vgl. Abschnitt 4.7), die im engeren Untersuchungsgebiet aber relativ selten sind, so dass wir einstweilen von einer formalen Beschreibung abgesehen haben (Einheit 2.3 in Tab. 15). Auf Ordnungs- und Verbandsebene spiegelt unser Gliederungsvorschlag floristische Gradienten in Abhängigkeit von Reliefposition, $\mathrm{pH}-$ Wert und Landnutzung wieder (Tab. 15). Mit dem Ver- 
band Grielo humifusi-Galenion sarcophyllae haben wir vermutlich erstmals im südlichen Afrika eine Gruppe anthropogener Ersatzvegetationstypen formal beschrieben, hier die Einheiten, die sich bei Überweidung ausbilden. Von den unterschiedenen Verbänden wies das Berkheyo fruticosae-Rhoion undulatae, das an felsigen Hängen und damit an den edaphisch feuchtesten und zugleich kleinräumig heterogensten Standorten wächst, mit im Mittel 30,0 Gefäßpflanzenarten auf $100 \mathrm{~m}^{2}$ die höchste $\alpha$-Diversität auf. Dagegen waren die Quarzfelder mit extrem salzigen und sauren Böden (Einheit 2.3 in Tab. 15) mit im Mittel nur 11,8 Arten pro $100 \mathrm{~m}^{2}$ am artenärmsten.

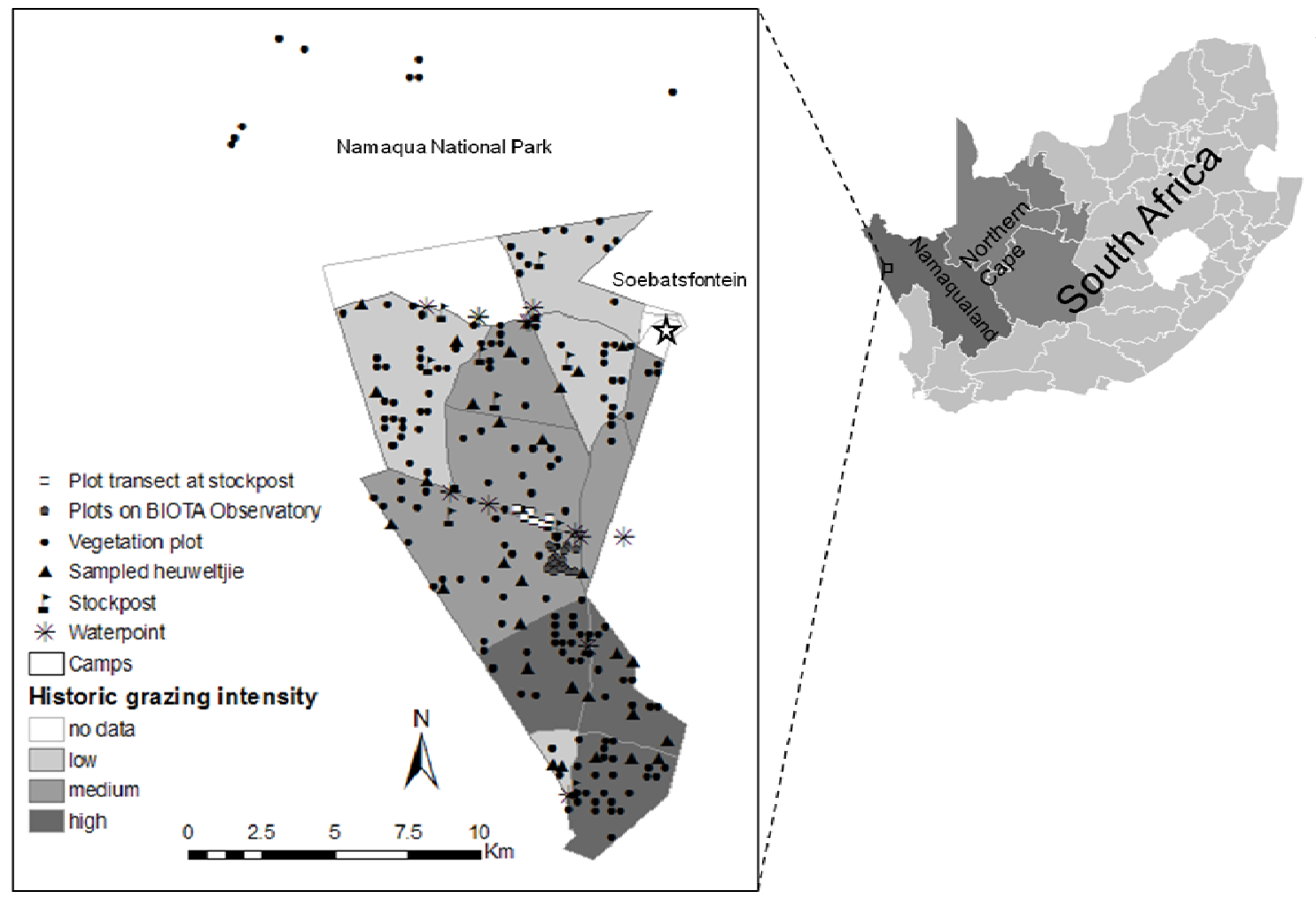

Abb. 39: Lage des Untersuchungsgebietes und Verteilung der Aufnahmeflächen der Klassifikationsstudie in der Hardeveld-Region der Sukkulenten Karoo (aus Luther-Mosebach et al. im Druck [38]). Die einzelnen Symbole entsprechen oftmals mehreren benachbarten Aufnahmeflächen.

Insgesamt zeigte sich, dass sich aktuelle pflanzensoziologischen Verfahren (vgl. Abschnitt 5.1) sehr wohl auch in einer natürlichen oder zumindest sehr naturnahen Vegetation hervorragend anwenden lassen, und nicht nur, wie manchmal behauptet, in einer durch scharfe anthropogene Nutzungsgrenzen geprägten Landschaft wie jener Mitteleuropas. Tatsächlich sind die von uns beschriebenen Syntaxa meist besser floristisch charakterisiert als auf der gleichen syntaxonomischen Ebene angesiedelte Einheiten in Europa. Ein Vergleich mit den wenigen anderen deskriptiven Vegetationsstudien aus der Sukkulenten Karoo zeigte, dass die beiden von uns beschriebe- 
nen Klassen im ganzen Biom verbreitet sind, und die in anderen Bereichen des Bioms beschriebenen informellen Einheiten zumindest teilweise zu den auch in unserer Studienregion vorkommenden Verbänden und Ordnungen gehören. Dagegen gab es auf der Assoziationsebene praktisch keine Übereinstimmungen selbst in nur 70-150 km entfernten Gebieten, was die extrem hohe $\beta$-Diversität des Bioms auch auf dieser Skalenebene unterstreicht (vgl. die Analysen zur $\beta$ Diversität bis zu $1 \mathrm{~km}^{2}$ in Abschnitt 4.6: Abb. 22).

Tab. 15: Vorgeschlagene syntaxonomische Gliederung der Hardeveld-Vegetation in der Sukkulenten Karoo (modifiziert aus Luther-Mosebach et al. im Druck [38]). Die Einheit 2.3 dürfte einer dritten Klasse entsprechen, wurde vorerst aber mangels genügend Aufnahmematerials nicht formal beschrieben und untergliedert. Heuweljies sind meist kreisrunde, fossile Termitarien, die bestimmten Bereichen der Sukkulenten Karoo ein schon von Weitem sichtbares, charakteristisches Vegetationsmuster verleihen, mit einer starken floristischen und edaphischen Differenzierung zwischen den Termitarien und der sie umgebenden Matrix.

\begin{tabular}{|c|c|c|c|c|c|c|}
\hline \multicolumn{4}{|c|}{ Klasse 1: Hermannio trifurcae-Zygophylletea morgsanae } & \multicolumn{3}{|c|}{$\begin{array}{l}\text { Klasse 2: Didelto carnosae-Cephalophylletea inae- } \\
\text { qualis }\end{array}$} \\
\hline \multicolumn{3}{|c|}{$\begin{array}{l}\text { Ordnung 1.1: } \\
\text { Hermannio trifurcae-Othonnetalia sedifoliae }\end{array}$} & \multirow{2}{*}{$\begin{array}{l}\text { Ordnung } \\
\text { 1.2: } \\
\text { Berkheyo } \\
\text { fruticosae- } \\
\text { Rhoetalia } \\
\text { undulatae } \\
\text { Verband } \\
\text { 1.2.1: Berk- } \\
\text { heyo frutico- } \\
\text { sae-Rhoion } \\
\text { undulatae }\end{array}$} & \multirow{2}{*}{$\begin{array}{c}\text { Ordnung 2.1: } \\
\text { Mesembry- } \\
\text { anthemo gueri- } \\
\text { chani- } \\
\text { Eberlanzietalia } \\
\text { cyathiformis } \\
\text { Verband 2.1.1: } \\
\text { Mesembry- } \\
\text { anthemo gueri- } \\
\text { chani- } \\
\text { Eberlanzion } \\
\text { cyathiformis }\end{array}$} & \multirow{2}{*}{$\begin{array}{c}\text { Ordnung 2.2: } \\
\text { Euphorbio hama- } \\
\text { tae- } \\
\text { Cephalophylletalia } \\
\text { inaequalis } \\
\\
\text { Verband 2.2.1: } \\
\text { Euphorbio hama- } \\
\text { tae-Cephalophyllion } \\
\text { inaequalis }\end{array}$} & \multirow[t]{2}{*}{$\begin{array}{l}\text { Einheit 2.3: } \\
\text { ohne Namen }\end{array}$} \\
\hline $\begin{array}{l}\text { Verband } \\
\text { 1.1.1: } \\
\text { Othonnion } \\
\text { cylindricae }\end{array}$ & $\begin{array}{l}\text { Verband } \\
\text { 1.1.2: } \\
\text { Stipagrostio } \\
\text { obtusae- } \\
\text { Othonnion } \\
\text { sedifoliae }\end{array}$ & $\begin{array}{l}\text { Verband } \\
\text { 1.1.3: } \\
\text { Grielo humifu- } \\
\text { si-Galenion } \\
\text { sarcophyllae }\end{array}$ & & & & \\
\hline $\begin{array}{l}\text { Tiefgründige, } \\
\text { rote Sande; } \\
\text { gering salin, } \\
\text { neutrale } \\
\text { Böden }\end{array}$ & $\begin{array}{l}\text { Sanddünen; } \\
\text { gering saline, } \\
\text { neutrale } \\
\text { Böden }\end{array}$ & $\begin{array}{l}\text { Flachgründige, } \\
\text { sandige Stan- } \\
\text { dorte; gering } \\
\text { saline, neutra- } \\
\text { le Böden; } \\
\text { gestörte Habi- } \\
\text { tate (intensiv } \\
\text { beweidet) }\end{array}$ & $\begin{array}{l}\text { Felsige Hän- } \\
\text { ge und Kup- } \\
\text { pen; leicht } \\
\text { saure Böden }\end{array}$ & $\begin{array}{l}\text { Zentren der } \\
\text { Heuweltjies und } \\
\text { ähnliche Vegeta- } \\
\text { tion; hoch saline, } \\
\text { leicht alkalische } \\
\text { Böden }\end{array}$ & $\begin{array}{l}\text { Matrixvegetation in } \\
\text { Heuweltjie- } \\
\text { Bereichen; hoch } \\
\text { saline, leicht saure } \\
\text { Böden }\end{array}$ & $\begin{array}{l}\text { Quarzfelder; } \\
\text { extrem sali- } \\
\text { ne, saure } \\
\text { Böden }\end{array}$ \\
\hline $\begin{array}{l}12 \text { Aufnah- } \\
\text { men }\end{array}$ & $\begin{array}{l}19 \text { Aufnah- } \\
\text { men }\end{array}$ & 50 Aufnahmen & $\begin{array}{l}77 \text { Aufnah- } \\
\text { men }\end{array}$ & 94 Aufnahmen & 95 Aufnahmen & 8 Aufnahmen \\
\hline 1 Assoziation & 1 Assoziation & $\begin{array}{l}2 \text { Assoziatio- } \\
\text { nen }\end{array}$ & $\begin{array}{l}3 \text { Assoziatio- } \\
\text { nen }\end{array}$ & 4 Assoziationen & 5 Assoziationen & $\begin{array}{l}\text { keine Asso- } \\
\text { ziation be- } \\
\text { schrieben }\end{array}$ \\
\hline
\end{tabular}




\section{Resümee und Ausblick}

In dieser Arbeit habe ich einen weiten Bogen durch verschiedene Felder der Biodiversitätsforschung gespannt und - so hoffe ich - an verschiedenen Stellen, einen Beitrag zur Fortentwicklung der Methoden, zum wissenschaftlichen Erkenntnisgewinn und/oder zur Verbesserung praktischer Umsetzung des gewonnenen Wissens geleistet. Ich habe dabei meine Publikationen aus vier großen Themenblöcken vorgestellt, wobei die zahlreichen Querverweise im Text deren vielfältige Verknüpfungen aufzeigen. Die hier zusammengeführten Arbeiten betrafen zahlreiche Facetten von Biodiversität sowie unterschiedliche Artengruppen, Ökosysteme und geografische Regionen (incl. virtueller Landschaften). Doch gibt es einige Schlüsselerkenntnisse, die sich durch viele meiner Arbeiten ziehen und die ich hier daher noch einmal zusammenfassend hervorheben will:

1. Biodiversität hat viele Facetten. Die sowohl in der wissenschaftlichen Diskussion als erst recht im Anwendungsbereich, etwa wenn es um Biodiversitätsschutz geht, zu findende Verengung auf einen oder wenige Aspekte ist nicht zielführend. Es wird nur derjenige zu einem umfassenden Bild kommen, der sich auf die Diversität von Biodiversität einlässt. Dies gilt insbesondere für die folgenden drei von mir in verschiedenen Arbeiten thematisierten Aspekte:

- Oftmals verhalten sich die verschiedenen Facetten von Biodiversität nicht kongruent, etwa $\alpha$ - vs. $\beta$-Diversität, Artenreichtum vs. Evenness, Diversität aller Arten vs. Diversität von Endemiten (vgl. Abschnitte 4.3-4.7).

- Ebenso unterscheiden sich die Biodiversitätsmuster verschiedener Taxa oft erheblich, weswegen die sowohl in der Forschung als auch im Naturschutz oft vorzufindende „Blickverengung“ auf Wirbeltiere und Gefäßpflanzen sehr problematisch ist. So machen Moose und Flechten einen erheblichen Anteil der botanischen Diversität aus, in manchen Ökosystemen sogar die Mehrheit. Daher lohnt es sich, sie in botanische Biodiversitätsanalysen einzubeziehen (vgl. Abschnitte 4.1, 4.4 und 4.5). Das Testen ökologischer Theorien an drei so verschiedenen Großtaxa verspricht ein viel tieferes Systemverständnis als die alleinige Betrachtung von Gefäßpflanzen. Wie ich gezeigt habe, können die NichtGefäßpflanzen auch praktische Bedeutung in Bereichen haben, wo diese bislang nicht so wahrgenommen wurde, etwa bei der Vegetationsklassifikation (vgl. Abschnitte 5.1 und $5.2)$.

- Die Skalenabhängigkeit im engeren Sinne, also Abhängigkeit von Biodiversitätsmustern und der sie bedingenden Faktoren von der betrachteten Korngröße (grain) ist ein bislang 
in der ökologischen Forschung und erst recht in der Anwendung weitgehend negiertes Phänomen. Ich habe gezeigt, dass diese Abhängigkeit sehr bedeutsam ist und einige als universell geltende „Muster“ (z. B. hemiboreale Zone ist artenärmer als temperate Zone oder Fynbos ist das Biom mit der höchsten Phytodiversität im südlichen Afrika) tatsächlich nicht allgemeingültig, sondern skalenabhängig sind (vgl. Abschnitte 4.6 und 5.2). Diese Erkenntnis sollte eine Warnung sein, Erkenntnisse ungeprüft von einer Skalenebene auf eine andere zu übertragen. Zugleich eröffnet das Studium dieser Skalenabhängigkeit vermutlich auch neue Wege zum Verständnis der treibenden Kräfte makroökologischer Muster.

2. Biodiversitätsforschung wie auch Biodiversitätsschutz sind auf eine gute Datengrundlage angewiesen (was nicht heißen soll, dass Biodiversitätsschutz warten sollte, bis die Datengrundlage besser ist).

- Es ist erschreckend, wie wenige konsistent über große geografische Räume und längere Zeiträume erhobene Biodiversitätsdaten es wirklich gibt. Hier ist es dringend geboten, international an einem Strang zu ziehen und globale Monitoringprogramme der verschiedenen Facetten von Biodiversität mit standarisierten Methoden lokal zu implementieren (vgl. Abschnitt 2.1).

- Die jetzt in großen Vegetationsdatenbanken verfügbar werdenden Datenmengen sind ein riesiger „Schatz“, der neue Analysemöglichkeiten für die botanische Biodiversitätsforschung verspricht, vorausgesetzt man ist sich der fast allen solchen Datenbanken inherenten Probleme (nicht-randomisierte Erhebung, verschiedene Plotgrößen, inkonsistente Sippennomenklatur) bewusst und sucht gezielt nach methodischen Lösungen für diese (vgl. Abschnitte 2.2 und 3.5.

3. In meinen methodisch-theoretischen Arbeiten hat mich immer wieder erstaunt, wie oft methodisch fragwürdige oder falsche Ergebnisse trotz der Qualitätssicherung durch das peer review-Verfahren publiziert und manchmal ganze Theorien darauf aufgebaut werden, die sogar Eingang in Lehrbücher finden. Wichtige Beispiele hierfür sind

- die irreführende Gleichsetzung von SARs und SSRs (vgl. Abschnitte 3.1 und 3.4),

- die falsche Annahme, dass sich SARs im Bereich von Pflanzengesellschaften am besten mit der Logarithmusfunktion beschreiben lassen (vgl. Abschnitt 4.1),

- die falsche Behauptung, dass der small island effect häufig auftritt (vgl. Abschnitt 3.3), oder

- die falsche Annahme, dass unterschiedliche Flächengrößen keinen wesentlichen Effekt bei auf Stetigkeiten basierenden Analysen habe (vgl. Abschnitt 3.5). 
Biodiversiätsforschung ist ein sehr komplexes und theoretisch wie methodisch anspruchsvolles Feld. Hier sind umso mehr kritische Aufmerksamkeit und das Hinterfragen von scheinbar gut belegten Theorien gefragt, wenn man diese Disziplin weiterbringen will. 


\section{Danksagung}

Gute Wissenschaft braucht kreative Freiräume und den intensiven Austausch mit Kolleginnen und Kollegen aus der gleichen und aus Nachbardisziplinen. Wenn ich auf die vier Jahre in der Abteilung Biodiversität, Evolution und Ökologie der Pflanzen an der Universität Hamburg zurückschaue, so waren dies die bislang mit Abstand innovativsten und produktivsten meiner wissenschaftlichen Laufbahn. Mein herzlicher Dank gilt daher Prof. Dr. Norbert Jürgens einerseits für die Aufnahme in seine Arbeitsgruppe, andererseits dafür, mir in Forschung und Lehre sehr große Freiräume gewährt zu haben, die gleichermaßen die Fortführung und Begründung neuer eigener Forschungsstränge wie auch die Mitwirkung an den Forschungs- und Publikationstätigkeiten der Arbeitsgruppe in verschiedenen Regionen Afrikas einschlossen. Mein herzlicher Dank gilt allen Mitgliedern der Arbeitsgruppe, die dafür gesorgt haben, dass ich mich vom ersten Tag an in Hamburg wohl fühlte. Besonders zu nennen sind hier meine wissenschaftlichen KollegInnen Dr. Ute Schmiedel, Dr. Manfred Finckh, Dr. Jens Oldeland, Daniela Haarmeyer, Dirk Wesuls, Dr. Gerhard Muche, Jona Luther-Mosebach, Niels Dreber, Rasmus Revermann, Tene Kwetche Sop, Wiebke Hanke und Zakia Akasbi, mit denen ich in den Jahren viele anregende Diskussionen hatte, die oftmals auch zur gemeinsamen Betreuung von Abschlussarbeiten oder zu gemeinsamen Publikationen führten. Annegret Saphir, Karen Dehn, Monika Petersen, Imke Oncken, Thomas Hillmann, Andrzej Suwald und Dr. Ingo Homburg unterstützten mich in vielerlei organisatorischer Hinsicht, wofür ich ihnen herzlich dankbar bin.

Meine wissenschaftliche Laufbahn wurde wesentlich durch zwei Kollegen beeinflusst, ohne die ich wohl nicht da angekommen wäre, wo ich jetzt stehe. Prof. Dr. Klaus Dierßen (Kiel), mein Diplom- und Doktorvater, hat mich geprägt, indem ich (a) von seiner herausragenden Artenkenntnis und seinem tiefen Verständnis ökologischer Systeme über viele Jahre lernen durfte, (b) er mir große Freiheiten ließ, meinen eigenen Weg zu finden, mich dabei aber immer seiner Rückendeckung versicherte, und (c) mich lehrte, Autoritäten zu misstrauen, dabei aber nicht selbst doktrinär zu werden. PD Dr. Christian Berg (Graz, vormals Rostock) verdanke ich, dass er mich Ende der 1990er Jahre als Co-Leiter des Projektes „Pflanzengesellschaften MecklenburgVorpommerns“ engagierte, aus dem nicht nur zahlreiche wissenschaftliche „Produkte“ entsprungen sind, sondern auch eine freundschaftlich-kollegiale Beziehung, die uns seit vielen Jahren immer wieder zusammenbringt.

Die hier vorgelegte Habilitationsschrift wäre nicht möglich gewesen ohne die intensive Kooperation mit zahlreichen Kolleginnen und Kollegen aus dem In- und Ausland. Allen meinen Koautorinnen danke ich deshalb für intensive, manchmal auch kontroverse, letztlich aber immer sachorientierte Diskussionen, die zu gemeinsamen Publikationen führten, die sich sehen lassen 
können. Besonders erwähnen möchte ich hier die von mir (mit-) betreuten Diplom-, Staatsexamens- und BachlorkandidatInnen. Ich hatte das unglaubliche Glück, in all den Jahren nur sehr gute und hoch motivierte KandidatInnen zu haben, was dazu beitrug dass wir nach Abgabe der Arbeiten diese meist gut publizieren konnten, aber auch dass ich mit vielen der ehemaligen KandidatInnen auch Jahre später noch freundschaftlich verbunden bin. Namentlich hervorheben möchte ich hier Dr. Swantje Löbel (Uppsala und Adenbüttel), Dr. Steffen Boch (Bern) und Daniela Haarmeyer (Frankfurt) für ihre herausragenden Diplomarbeiten, die jeweils gleich zu mehreren in diese Habilitationsschrift einbezogenen hochrangigen Publikationen führten. Vielen Dank für die gute und enge Zusammenarbeit und dafür, dass wir teilweise noch Jahre nach Ende der Diplomarbeit daraus gemeinsam spannende wissenschaftliche „Stories“ gemacht haben (und noch machen).

Von der Universität Hamburg danke ich neben Prof. Dr. Norbert Jürgens auch Prof. Dr. Kai Jensen und Prof. Dr. Jörg Ganzhorn für die wohlwollende Unterstützung meiner Habilitation und für die „drängende“ Ermunterung, das „Opus“ endlich zum Abschluss zu bringen. Dr. Jens Oldeland hat dankenswerterweise eine frühere Fassung dieser Arbeit kritisch durchgesehen und mit seinen Anregungen zu ihrer Optimierung beigetragen.

Schließlich und ganz besonders gilt mein tiefer Dank meinen Eltern, Doris und Reinhart Dengler, die meinen beruflichen Weg seit Beginn des Studium in jeder Hinsicht gefördert und unterstützt haben, gerade auch in Zeiten, in denen er nicht gerade verlief, sondern von Stagnation und Zweifeln oder Arbeitslosigkeit unterbrochen war. 


\section{Literatur}

\subsection{Vorgelegte eigene Publikationen}

\section{Quellen von Biodiversitätsdaten}

[1] Dengler, J. (2009b): A flexible, multi-scale approach for standardised recording of plant species richness patterns. - Ecological Indicators 9: 1169-1178.

[2] Jürgens, N., Schmiedel, U., Haarmeyer, D. H., Dengler, J., Finckh, M., Goetze, D., Gröngröft, A., Hahn, K., Koulibaly, A., Luther-Mosebach, J., Muche, G., Oldeland, J., Petersen, A., Porembski, S., Rutherford, M. C., Schmidt, M., Sinsin, B., Strohbach, B. J., Thiombiano, A., Wittig, R., Zizka, G. (im Druck): The BIOTA Biodiversity Observatories in Africa - A standardized framework for large-scale environmental monitoring. - Environmental Monitoring and Assessment. DOI: 10.1007/s10661-011-1993-y.

[3] Dengler, J., Ewald, J., Kühn, I., Peet, R. K. (2011a): Ecoinformatics and global change - an overdue liaison. - Journal of Vegetation Science 22: 577-581.

[4] Dengler, J., Jansen, F., Glöckler, F., Peet, R. K., De Cáceres, M., Chytrý, M., Ewald, J., Oldeland, J., Finckh, M., Lopez-Gonzalez, G., Mucina, L., Rodwell, J. S., Schaminée, J. H. J., Spencer, N. (2011b): The Global Index of Vegetation-Plot Databases (GIVD): a new resource for vegetation science. - Journal of Vegetation Science 22: 582-597.

[5] Jansen, F., Dengler, J., Glöckler, F., Chytrý, M., Ewald, J., Oldeland, J., Schaminée, J. H. J. (2011): Die mitteleuropäischen Datenbanken im Global Index of Vegetation-Plot Databases (GIVD). - Tuexenia 31: $351-367$.

[6] Jansen, F., Dengler, J. (2008): GermanSL - Eine universelle taxonomische Referenzliste für Vegetationsdatenbanken in Deutschland. - Tuexenia 28: 239-253.

[7] Jansen, F., Dengler, J. (2010): Plant names in vegetation databases - a neglected source of bias. - Journal of Vegetation Science 21: 1179-1186.

\section{Theorie, Simulationen und Analysemethoden zur Skalenabhängigkeit von Biodiversität}

[8] Dengler, J. (2009a): Which function describes the species-area relationship best? - A review and empirical evaluation. - Journal of Biogeography 36: 728-744.

[9] Dengler, J. (2008): Pitfalls in small-scale species-area sampling and analysis. - Folia Geobotanica 43: 269287.

[10] Dengler, J., Oldeland, J. (2010): Effects of sampling protocol on the shapes of species richness curves. Journal of Biogeography 37: 1698-1705.

[11] Dengler, J., Boch, S. (2008b): Sampling-design effects on properties of species-area curves - a case study from Estonian dry grassland communities. - Folia Geobotanica 43: 289-304.

[12] Dengler, J. (2010): Robust methods for detecting a small island effect. - Diversity and Distributions 16: 256266.

[13] Dengler, J., Löbel, S., Dolnik, C. (2009): Species constancy depends on plot size - a problem for vegetation classification and how it can be solved. - Journal of Vegetation Science 20: 754-766. 


\section{Artendiversität auf verschiedenen Skalenebenen}

[14] Löbel, S., Dengler, J., Hobohm, C. (2004): Beziehungen zwischen der Artenvielfalt von Gefäßpflanzen, Moosen und Flechten in Trockenrasen der Insel Öland (Schweden). - Kieler Notizen zur Pflanzenkunde Schleswig-Holstein und Hamburg 32: 9-13.

[15] Dengler, J. (2005): Zwischen Estland und Portugal - Gemeinsamkeiten und Unterschiede der Phytodiversitätsmuster europäischer Trockenrasen. - Tuexenia 25: 387-405.

[16] Allers, M.-A., Dengler, J. (2007): Small-scale patterns of plant species richness in the central European landscape. - Verhandlungen der Gesellschaft für Ökologie 37: 181.

[17] Dengler, J. (2006): Variabilität von Artendichte und Artenzusammensetzung auf unterschiedlichen räumlichen Skalenebenen - Exemplarische Untersuchungen aus Trockenrasen und Konsequenzen für das Probedesign von Biodiversitätsuntersuchungen. - In: Bültmann, H., Fartmann, T., Hasse, T. [Eds.]: Trockenrasen auf unterschiedlichen Betrachtungsebenen. - Arbeiten aus dem Institut für Landschaftsökologie Münster 15: 7381. Münster.

[18] Turtureanu, P. D., Dengler, J. (im Druck): Different aspects of plant diversity show contrasting patterns in Carpathian forest openings. - Plant Ecology. DOI: 10.1007/s11258-011-0007-z.

[19] Löbel, S., Dengler, J., Hobohm, C. (2006): Species richness of vascular plants, bryophytes and lichens in dry grasslands: The effects of environment, landscape structure and competition. - Folia Geobotanica 41: 377393.

[20] Ruprecht, E., Szabó, A., Enyedi, M. Z., Dengler, J. (2009): Steppe-like grasslands in Transylvania (Romania): characterisation and influence of management on species diversity and composition. - Tuexenia 29: 353-368 + 1 Tabelle.

[21] Vassilev, K., Pedashenko, H., Nikolov, S. C., Apostolova, I., Dengler, J. (2011): Effect of land abandonment on the vegetation of upland semi-natural grasslands in the Western Balkan Mts., Bulgaria. - Plant Biosystems 145: 654-665.

[22] Friedel, A., Oheimb, G. von, Dengler, J., Härdtle, W. (2006): Species diversity and species composition of epiphytic bryophytes and lichens - a comparison or managed and unmanaged beech forests in NE Germany. - Feddes Repertorium 117: 172-185.

[23] Schmiedel, U., Dengler, J., Luther-Mosebach, J., Gröngröft, A., Muche, G., Petersen, A., Strohbach, B. J., Jürgens, N. (2010): Patterns and dynamics of vascular plant diversity along the BIOTA transects in southern Africa. - In: Schmiedel, U., Jürgens, N. [Eds.]: Biodiversity in Southern Africa. Volume 2: Patterns and Processes at Regional Scale: pp. 118-135. Klaus Hess Publishers, Göttingen \& Windhoek.

[24] Haarmeyer, D. H., Schmiedel, U., Dengler, J., Bösing, B. M. (2010a): How does grazing intensity affect different vegetation types in arid Succulent Karoo, South Africa? Implications for conservation management. - Biological Conservation 143: 588-596.

[25] Schmiedel, U., Dengler, J., Etzold, S. (im Druck): Vegetation dynamics of endemic-rich quartz fields in the Succulent Karoo, South Africa, in response to recent climatic trends. - Journal of Vegetation Science. DOI: 10.1111/j.1654-1103.2011.01346.x.

\section{Analyse und Diversität von Pflanzengesellschaften}

[26] Dengler, J., Chytrý, M., Ewald, J. (2008): Phytosociology. - In: Jørgensen, S. E., Fath, B. D. [Eds.]: Encyclopedia of Ecology: pp. 2767-2779. Elsevier, Oxford. 
[27] Dengler, J., Berg, C., Jansen, F. (2005): New ideas for modern phytosociological monographs. - Annali di Botanica N. S. 5: 49-66.

[28] Berg, C., Dengler, J. (2005): Moose und Flechten als diagnostische Arten von Pflanzengesellschaften - eine Übersicht aus Mecklenburg-Vorpommern. - Herzogia 18: 145-161.

[29] Timmermann, T., Dengler, J., Abdank, A., Berg, C. (2006): Objektivierung von Naturschutzbewertungen Das Beispiel Roter Listen von Pflanzengesellschaften. - Naturschutz und Landschaftsplanung 38: 133-139.

[30] Dengler, J., Löbel, S. (2006): The basiphilous dry grasslands of shallow, skeletal soils (Alysso-Sedetalia) on the island of Öland (Sweden), in the context of North and Central Europe. - Phytocoenologia 36: 343-391.

[31] Dengler, J., Löbel, S., Boch, S. (2006b): Dry grassland communities of shallow, skeletal soils (SedoScleranthenea) in northern Europe. - Tuexenia 26: 159-190 + 6 Tabellen.

[32] Boch, S., Dengler, J. (2006): Floristische und ökologische Charakterisierung sowie Phytodiversität der Trockenrasen auf der Insel Saaremaa (Estland). - In: Bültmann, H., Fartmann, T., Hasse, T. [Eds.]: Trockenrasen auf unterschiedlichen Betrachtungsebenen. - Arbeiten aus dem Institut für Landschaftsökologie Münster 15: 55-71. Münster.

[33] Löbel, S., Dengler, J. (2008) [,,2007’]: Dry grassland communities on southern Öland: phytosociology, ecology, and diversity. - In: van der Maarel, E. [Ed.]: Structure and dynamics of alvar vegetation on Öland and some related dry grasslands - Dedicated to Ejvind Rosén on his $65^{\text {th }}$ birthday. - Acta Phytogeographica Suecica 88: 13-32. Svenska Växtgeografiska Sällskapet, Uppsala.

[34] Dengler, J., Eisenberg, M., Schröder, J. (2006a): Die grundwasserfernen Saumgesellschaften Nordostniedersachsens im europäischen Kontext - Teil I: Säume magerer Standorte (Trifolio-Geranietea sanguinei). - Tuexenia 26: 51-93 + 9 Tabellen.

[35] Dengler, J., Eisenberg, M., Schröder, J. (2007): Die grundwasserfernen Saumgesellschaften Nordostniedersachsens im europäischen Kontext - Teil II: Säume nährstoffreicher Standorte (Artemisietea vulgaris) und vergleichende Betrachtung der Saumgesellschaften insgesamt. - Tuexenia 27: 91-136 + 6 Tabellen.

[36] Dengler, J., Boch, S. (2008a): Forest-edge communities (Trifolio-Geranietea sanguinei) on the island of Saaremaa (Estonia): Phytosociology and biodiversity patterns. - In: Dengler, J., Dolnik, C., Trepel, M. [Eds.]: Flora, Vegetation und Naturschutz zwischen Schleswig-Holstein und Südamerika - Festschrift für Klaus Dierßen zum 60. Geburtstag. - Mitteilungen der Arbeitsgemeinschaft Geobotanik in SchleswigHolstein und Hamburg 65: 257-286 + 2 Beilagen. Kiel.

[37] Michl, T., Dengler, J., Huck, S. (2010): Montane-subalpine tall-herb vegetation (Mulgedio-Aconitetea) in central Europe: large-scale synthesis and comparison with northern Europe. - Phytocoenologia 40: 117-154.

[38] Luther-Mosebach, J., Dengler, J., Schmiedel, U., Röwer, I. U., Labitzky, T., Gröngröft, A. (im Druck): A first formal classification of Hardeveld vegetation in Namaqualand, South Africa. - Applied Vegetation Science. DOI: 10.1111/j.1654-109X.2011.01173.x.

\subsection{Alle zitierten Quellen in alphabetischer Reihenfolge}

Adler, P. B., White, E. P., Lauenroth, W. K., Kaufman, D. M., Rassweiler, A., Rusak, J. A. (2005): Evidence for a general species-time-area relationship. - Ecology 86: 2032-2039.

AGP [The Angiosperm Phylogeny Group] (2009): An update of the Angiosperm Phylogeny Group classification for the orders and families of flowering plants: AGP III. - Botanical Journal of the Linnean Society 161: 105-121. 
Albertson, N. (1950): Der grosse südliche Alvar der Insel Öland - Eine pflanzensoziologische Übersicht. - Svensk Botanisk Tidskrift 44: 270-331 + 4 Tafeln.

Allers, M.-A., Dengler, J. (2007): Small-scale patterns of plant species richness in the central European landscape.Verhandlungen der Gesellschaft für Ökologie 37: 181.

Anderson, M. J., Crist, T. O., Chase, J. M., Vellend, M., Inouye, B. D., Freestone, A. L., Sanders, N. J., Cornell, H. V., Comita, L. S., Davies, K. F., Harrison, S. P., Kraft, N. J. B., Stegen, J. C., Swenson, N. G. (2011): Navigating the multiple meanings of $\beta$ diversity: a roadmap for the practicing ecologist. - Ecology Letters 14: $19-28$.

Arrhenius, O. (1920): Distribution of the species over the area. - Meddelanden från K Vetenskapsakademiens Nobelinstitut 4(7): 1-6.

Ascherson, P. (1864): Flora der Provinz Brandenburg, der Altmark und des Herzogthums Magdeburg. - XII + 146 +1034 pp. Hirschwald, Berlin.

Azovsky, A. I. (2011): Species-are and species-sampling effort relationships: disentangling the effects. - Ecography 34: $18-30$.

Balmford, A., Bennun, L., ten Brink, B., Cooper, D., Côté, I. M., Crane, P., Dobson, A., Dudley, N., Dutton, I., Green, R. E., Gregory, R. D., Harrison, J., Kennedy, E. T., Kremen, C., Leader-Williams, N., Lovejoy, T. E., Mace, G., May, R., Mayaux, P., Morling, P., Redford, K., Ricketts, T. H., Rodríguez, J. P., Sanjayan, M., Schei, P. J., van Jaarsveld, A. S., Walther, B. A. (2005): The Convention on Biological Diversity's 2010 target. Science 307: 212-213.

Barkman, J. J. (1989): A critical evaluation of minimum area concepts. - Vegetatio 85: 89-104.

Barthlott, W., Mutke, J., Rafiqpoor, D., Kier, G., Kreft, H. (2005): Global centers of vascular plant diversity. - Nova Acta Leopoldina N. F. 92: 61-83.

Berendsohn, W. G. (1995): The concept of "potential taxa" in databases. - Taxon 44: 207-212.

Berg, C., Dengler, J. (2005): Moose und Flechten als diagnostische Arten von Pflanzengesellschaften - eine Übersicht aus Mecklenburg-Vorpommern. - Herzogia 18: 145-161.

Berg, C., Dengler, J., Abdank, A. (2001) [Eds.]: Die Pflanzengesellschaften Mecklenburg-Vorpommerns und ihre Gefährdung - Tabellenband. - 341 pp., Weissdorn, Jena.

Berg, C., Dengler, J., Abdank, A., Isermann, M. (2004) [Eds.]: Die Pflanzengesellschaften MecklenburgVorpommerns und ihre Gefährdung - Textband. - 606 pp., Weissdorn, Jena.

Bergmeier, E., Härdtle, W., Mierwald, U., Nowak, B., Peppler, C. (1990): Vorschläge zur syntaxonomischen Arbeitsweise in der Pflanzensoziologie. - Kieler Notizen zur Pflanzenkunde in Schleswig-Holstein und Hamburg 20: $92-110$.

BFANL [Bundesforschungsanstalt für Naturschutz und Landschaftsökologie] (1986) [Ed.]: Rote Listen von Pflanzengesellschaften, Biotopen und Arten. - Schriftenreihe für Vegetationskunde 18: 166 pp. BFANL, Bonn.

Bisby, F. A. (2000): The quiet revolution: biodiversity informatics and the internet. - Science 289: $2309-2312$.

Bischoff, C., Dröschmeister, R. (2000) [Eds.]: European Monitoring for Nature Conservation. - Schriftenreihe für Lanschaftspflege und Naturschutz 62: 199 pp. Bundesamt für Naturschutz, Bonn.

Blab, J., Riecken, U. (1993) [Eds.]: Grundlagen und Probleme einer Roten Liste der gefährdeten Biotoptypen Deutschlands: Referate und Ergebnisse des gleichnamigen Symposiums der Bundesforschungsanstalt für Naturschutz und Landschaftsökologie vom 28.-30. Oktober 1991. - Schriftenreihe für Landschaftspflege und Naturschutz 38: 339 pp. Kilda, Greven. 
Blackburn, T. M., Gaston, K. J. (2003) [Eds.]: Macroecology: Concepts and Consequences - The 43rd Annual Symposium of the British Ecological Society held at the University of Birmingham, 17-19 April 2002. - XVII + 422 pp., Blackwell, Malden, MA.

Boch, S. (2005): Phytodiversität, Charakterisierung und Syntaxonomie der Trockenrasen auf Saaremaa (Estland). II + 89 + IX pp. + Beilagemappe. Diplomarbeit, Institut für Ökologie und Umweltchemie, Universität Lüneburg. URL: http://www.biologie.uni-hamburg.de/bzf/syst/Diplomarbeit_Steffen_Boch_2005.pdf.

Boch, S., Dengler, J. (2006): Floristische und ökologische Charakterisierung sowie Phytodiversität der Trockenrasen auf der Insel Saaremaa (Estland). - In: Bültmann, H., Fartmann, T., Hasse, T. [Eds.]: Trockenrasen auf unterschiedlichen Betrachtungsebenen. - Arbeiten aus dem Institut für Landschaftsökologie Münster 15: 55-71. Münster.

Bohn, U., Gollub, G., Hettwer, C., Neuhäuslová, Z., Raus, T., Schlüter, H., Weber, H., Hennekens, S. (2004) [Eds.]: Map of the Natural Vegetation of Europe. Scale $1: 2500$ 000. Interactive CD-ROM: Explanatory Text, Legend, Maps. - CD-ROM + 19 pp. Bundesamt für Naturschutz, Bonn.

Bossuyt, B., Hermy, M. (2004): Species turnover at small scales in dune slack plant communities. - Basic and Applied Ecology 5: 321-329, Jena.

Braun-Blanquet, J. (1963): Das Helianthemo-Globularion, ein neuer Verband der baltischen Steppenvegetation. Veröffentlichungen des Geobotanischen Instituts der Eidgenössischen Technischen Hochschule, Stiftung Rübel, in Zürich 37: 27-38.

Braun-Blanquet, J. (1964): Pflanzensoziologie - Grundzüge der Vegetationskunde. 3. Aufl. - XIV + 865 pp. Springer, Wien.

Brown, J. H. (1995): Macroecology. - 269 pp. University of Chicago Press, Chicago.

Brown, J. H., Maurer, B. A. (1989): Macroecology: the division of food and space among species on continents. Science 243: 1145-1150.

Bruelheide, H. (1995): Die Grünlandgesellschaften des Harzes und ihre Standortbedingungen. Mit einem Beitrag zum Gliederungsprinzip auf der Basis von statistisch ermittelten Artengruppen. - Dissertationes Botanicae 244: IX + 338 pp. + Tabellen A1-A11. Borntraeger, Berlin.

Bruelheide, H. (2000): A new measure of fidelity and its application to defining species groups. - Journal of Vegetation Science 11: 167-178.

Bruelheide, H., Jandt, U. (1997): Demarcation of communities in large databases. - Phytocoenologia 27: $141-159$.

Brunt, J. W., McCartney, P., Baker, K., Stafford, S. G. (2002): The future of ecoinformatics in long term ecological research. - In: Callaos, N. [Ed.]: Proceedings of the 6th World Multiconference on Systemics, Cybernetics and Informatics: SCI 2002 ; July 14-18, 2002 Orlando, Florida, USA. Volume 7: Information Systems Development, II: pp. 367-372. International Institute of Informatics and Systemics, Orlando, FL, US.

Burnham, K. P., Anderson, D. R. (2002): Model Selection and Multimodel Inference - a Practical InformationTheoretic Approach. 2. Aufl. - XXVI + 488 pp. Springer, New York.

Burns, K. C., McHardy, R. P., Pledger, S. (2009): The small-island effect: fact or artefact? - Ecography 32: 269276.

Butchart, S. H. M., Waldpole, M., Collen, B., van Strien, A., Scharlemann, J. P. W., Almond, R. E. A., Baillie, J. E. M., Bomhard, B., Brown, C., Bruno, J., Carpenter, K. E., Carr, G. M., Chanson, J., Chenery, A. M., Csirke, J., Davidson, N. C., Dentener, F., Foster, M., Galli, A., Galloway, J. N., Genovesi, P., Gregory, R. D., Hockings, M., Kapos, V., Lamarque, J.-F., Leverington, F., Loh, J., McGeoch, M. A., McRae, L., Minasyan, A., Hernández 
Morcillo, M., Oldfield, T. E. E., Pauly, D., Quader, S., Revenga, C., Sauer, J. R., Skolnik, B., Spear, D., Stanwell-Smith, D., Stuart, S. N., Symes, A., Tierney, M., Tyrrell, T. D., Vié, J.-C., Watson, R. (2010): Global biodiversity: indicators of recent declines. - Science 328: 1164-1168.

Cadotte, M. W., Davies, T. J., Regetz, J., Kembel, S. W., Cleland, E., Oakley, T. H. (2010): Phylogenetic diversity metrics for ecological communities: integrating species richness, abundance and evolutionary history. - Ecology Letters 13: 96-105.

Callicott, J. B. (2006): Conservation values and ethics. - In: Groom, M. J., Meffe, G. K., Carroll, C. R. [Eds.]: Principles of Conservation Biology. 3. Aufl.: pp. 111-135. Sinauer, Sunderland, MA.

Carranza, L., Feoli, E., Ganis, P. (1998): Analysis of vegetation structural diversity by Burnaby’s similarity index. Plant Ecology 138: 77-87.

Cavender-Bares, J., Kozak, K. H., Fine, P. V. A., Kembel, S. W. (2009): The merging of community ecology and phylogenetic biology. - Ecology Letters 12: 693-715, Oxford.

Chapin, F. S., III., Zavaleta, E. S., Eviners, V. T., Naylor,, R. L., Vitousek, P. M., Reynolds, H. L., Hooper, D. U., Lavorel, S., Sala, O. E., Hobbie, S. E., Mack, M. C., Díaz, S. (2000): Consequences of changing biodiversity. Nature 405: 234-242.

Chapman, A. D. (2009): Numbers of Living Species in Australia and the World. 2. Aufl. - 80 pp. Australian Biological Resources Study, Canberra.

Chiarucci, A., Bacaro, G., Rocchini, D., Ricotta, C., Palmer, M. W., Scheiner, S. M. (2009): Spatially constrained rarefaction: incorporating the autocorrelated structure of biological communities into sample-based rarefaction. Community Ecology 10: 209-214.

Chytrý, M. (2007) [Ed.]: Vegetation of the Czech Republic - 1. Grassland and Heathland Vegetation [tschechisch, mit englischen Zusammenfassungen]. - 526 pp. Academia, Praha.

Chytrý, M., Otýpková, Z. (2003): Plot sizes used for phytosociological sampling of European vegetation. - Journal of Vegetation Science 14: 563-570.

Chytrý, M., Tichý, L., Holt, J., Botta-Dukát, Z. (2002): Determination of diagnostic species with statistical fidelity measures. - Journal of Vegetation Science 13: 79-90.

Ciccarelli, F. D., Doerks,T., von Mering, C., Creevey, C. J., Snel, B., Bork, P. (2006): Toward automatic reocnstruction of a highly resolved tree of life. - Science 311: 1283-1287.

Collins, S. L., Glenn, S. M., Gibson, D. J. (1995): Experimental analysis of intermediate disturbance and initial floristic composition: Decoupling cause and effect. - Ecology 76: 486-492.

Colwell, R. K., Coddington, J. A. (1994): Estimating terrestrial biodiversity through extrapolation. - Philosophical Transactions of the Royal Society of London, B 345: 101-118.

Connell, J. H. (1978): Diversity in tropical rain forests and coral reefs. - Science 199: 1302-1310.

Connor, E. F., McCoy, E. D. (1979): The statistics and biology of the species-area relationship. - American Naturalist 113: 791-833.

Connor, E. F., McCoy, E. D. (2001): Species-area relationships. - In: Levin, S. A. [Ed.]: Encyclopedia of Biodiversity, Volume 5: pp. 397-411., Academic Press, San Diego.

Cornell, H. V., Karlson, R. H. (1997): Local and regional processes as controls of species richness. - In: Tilman, D., Kareiva, P. [Eds.]: Spatial ecology - the role of space in population dynamics and interspecific interactions. - In: Levin, S. A., Horn, H. S. [Eds.]: Monographs in Population Biology 30: pp. 250-268. Princeton University Press, Princeton. 
Crawley, M. J., Harral, J. E. (2001): Scale dependence in plant biodiversity. - Science 291: 864-868.

Crist, T. O., Veech, J. A. (2006): Additive partitioning of rarefaction curves and species-area relationships: unifying $\alpha$-, $\beta$ - and $\gamma$-diversity with sample size and habitat area. - Ecology Letters 9: 923-932.

Darwin, C. (1859): On the Origin of Species by Means of Natural Selection, or the Preservation of Favoured Races in the Struggle for Live. - 502 pp. Murray, London.

de Bello, F., Thuiller, W., Lepš, J., Choler, P., Clément, J.-C., Macek, P., Sebastià, M.-T., Lavorel, S. (2009): Partitioning of functional diversity reveals the scale and extent of trait convergence and divergence. - Journal of Vegetation Science 20: 475-486.

den Boer, P. J. (1986): The present status of the competitive exclusion principle. - Trends in Ecology \& Evolution 1: $25-28$.

Dengler, J. (2003): Entwicklung und Bewertung neuer Ansätze in der Pflanzensoziologie unter besonderer Berücksichtigung der Vegetationsklassifikation. - Archiv naturwissenschaftlicher Dissertationen 14: 297 pp. Galunder, Nümbrecht.

Dengler, J. (2004): Klasse: Koelerio-Corynephoretea Klika in Klika \& V. Novák 1941 - Sandtrockenrasen und Felsgrusfluren von der submeridionalen bis zur borealen Zone. - In: Berg, C., Dengler, J., Abdank, A., Isermann, M. [Eds.]: Die Pflanzengesellschaften Mecklenburg-Vorpommerns und ihre Gefährdung - Textband: pp. 301-326. Weissdorn, Jena.

Dengler, J. (2005): Zwischen Estland und Portugal - Gemeinsamkeiten und Unterschiede der Phytodiversitätsmuster europäischer Trockenrasen. - Tuexenia 25: 387-405.

Dengler, J. (2006): Variabilität von Artendichte und Artenzusammensetzung auf unterschiedlichen räumlichen Skalenebenen - Exemplarische Untersuchungen aus Trockenrasen und Konsequenzen für das Probedesign von Biodiversitätsuntersuchungen. - In: Bültmann, H., Fartmann, T., Hasse, T. [Eds.]: Trockenrasen auf unterschiedlichen Betrachtungsebenen. - Arbeiten aus dem Institut für Landschaftsökologie Münster 15: 73-81. Münster.

Dengler, J. (2008): Pitfalls in small-scale species-area sampling and analysis. - Folia Geobotanica 43: 269-287.

Dengler, J. (2009a): Which function describes the species-area relationship best? - A review and empirical evaluation. - Journal of Biogeography 36: 728-744.

Dengler, J. (2009b): A flexible, multi-scale approach for standardised recording of plant species richness patterns. Ecological Indicators 9: 1169-1178.

Dengler, J. (2010): Robust methods for detecting a small island effect. - Diversity and Distributions 16: $256-266$.

Dengler, J., Boch, S. (2008a): Forest-edge communities (Trifolio-Geranietea sanguinei) on the island of Saaremaa (Estonia): Phytosociology and biodiversity patterns. - In: Dengler, J., Dolnik, C., Trepel, M. [Eds.]: Flora, Vegetation und Naturschutz zwischen Schleswig-Holstein und Südamerika - Festschrift für Klaus Dierßen zum 60. Geburtstag. - Mitteilungen der Arbeitsgemeinschaft Geobotanik in Schleswig-Holstein und Hamburg 65: 257-286 + 2 Beilagen. Kiel.

Dengler, J., Boch, S. (2008b): Sampling-design effects on properties of species-area curves - a case study from Estonian dry grassland communities. - Folia Geobotanica 43: 289-304.

Dengler, J., Löbel, S. (2006): The basiphilous dry grasslands of shallow, skeletal soils (Alysso-Sedetalia) on the island of Öland (Sweden), in the context of North and Central Europe. - Phytocoenologia 36: 343-391.

Dengler, J., Oldeland, J. (2010): Effects of sampling protocol on the shapes of species richness curves. - Journal of Biogeography 37: 1698-1705. 
Dengler, J., Berg, C., Jansen, F. (2005): New ideas for modern phytosociological monographs. - Annali di Botanica N. S. 5: 49-66.

Dengler, J., Eisenberg, M., Schröder, J. (2006a): Die grundwasserfernen Saumgesellschaften Nordostniedersachsens im europäischen Kontext - Teil I: Säume magerer Standorte (Trifolio-Geranietea sanguinei). - Tuexenia 26: 51$93+9$ Tabellen.

Dengler, J., Löbel, S., Boch, S. (2006b): Dry grassland communities of shallow, skeletal soils (Sedo-Scleranthenea) in northern Europe. - Tuexenia 26: 159-190 + 6 Tabellen.

Dengler, J., Rūsiņa, S., Boch, S., Bruun, H. H., Diekmann, M., Dierßen, K., Dolnik, C., Dupré, C., Golub, V. B., Grytnes, J.-A., Helm, A., Ingerpuu, N., Löbel, S., Pärtel, M., Rašomavičius, V., Tyler, G., Znamenskiy, S. R., Zobel, M. (2006c): Working group on dry grasslands in the Nordic and Baltic region - Outline of the project and first results for the class Festuco-Brometea. - Annali di Botanica N. S. 6: 1-28.

Dengler, J., Eisenberg, M., Schröder, J. (2007): Die grundwasserfernen Saumgesellschaften Nordostniedersachsens im europäischen Kontext - Teil II: Säume nährstoffreicher Standorte (Artemisietea vulgaris) und vergleichende Betrachtung der Saumgesellschaften insgesamt. - Tuexenia 27: 91-136 + 6 Tabellen.

Dengler, J., Chytrý, M., Ewald, J. (2008): Phytosociology. - In: Jørgensen, S. E., Fath, B. D. [Eds.]: Encyclopedia of Ecology: pp. 2767-2779. Elsevier, Oxford.

Dengler, J., Löbel, S., Dolnik, C. (2009): Species constancy depends on plot size - a problem for vegetation classification and how it can be solved. - Journal of Vegetation Science 20: 754-766.

Dengler, J., Ewald, J., Kühn, I., Peet, R. K. (2011a): Ecoinformatics and global change - an overdue liaison. Journal of Vegetation Science 22: 577-581.

Dengler, J., Jansen, F., Glöckler, F., Peet, R. K., De Cáceres, M., Chytrý, M., Ewald, J., Oldeland, J., Finckh, M., Lopez-Gonzalez, G., Mucina, L., Rodwell, J. S., Schaminée, J. H. J., Spencer, N. (2011b): The Global Index of Vegetation-Plot Databases (GIVD): a new resource for vegetation science. - Journal of Vegetation Science 22: 582-597.

Desmet, P., Cowling, R. (2004): Using the species-area relationship to set baseline targets for conservation. Ecology \& Society 9(2): Artikel 1: 1-23. URL: http://www.ecologyandsociety.org/vol9/iss2/art11.

Diekmann, M. (1995): Delimitation of syntaxa in northern Europe - a case study. - Annali di Botanica 53: 65-79.

Diekmann, M. (1997): The differentiation of alliances in South Sweden. - Folia Geobotanica \& Phytotaxonomica 32: 193-205.

Dierschke, H. (1974): Saumgesellschaften im Vegetations- und Standortgefälle an Waldrändern. - Scripta Geobotanica 6: 246 pp. + 5 Tabellen. Goltze, Göttingen.

Dierschke, H. (1981): Zur syntaxonomischen Bewertung schwach gekennzeichneter Pflanzengesellschaften. - In: Dierschke, H. [Ed.]: Syntaxonomie (Rinteln 31.3.-3.4.1980). - Berichte der internationalen Symposien der Internationalen Vereinigung für Vegetationskunde 24: 109-122. Cramer, Vaduz.

Dierschke, H. (1994): Pflanzensoziologie - Grundlagen und Methoden - 683 pp. Ulmer, Stuttgart.

Dierßen, K. (1990): Einführung in die Pflanzensoziologie (Vegetationskunde). - 241 pp. Wissenschaftliche Buchgesellschaft, Darmstadt.

Dierßen, K., Dierßen, B. (1996): Vegetation Nordeuropas. - 838 pp. Ulmer, Stuttgart.

Dobson, A. (1995): Biodiversity and human health. - Trends in Ecology \& Evolution 10: 390-391.

Dolnik, C. (2003): Artenzahl-Areal-Beziehungen von Wald- und Offenlandgesellschaften - Ein Beitrag zur Erfassung der botanischen Artenvielfalt unter besonderer Berücksichtigung der Flechten und Moose am Beispiel 
des Nationalparks Kurische Nehrung (Russland). - Mitteilungen der Arbeitsgemeinschaft Geobotanik in Schleswig-Holstein und Hamburg 62: 183 pp. Kiel.

Dolnik, C., Breuer, M. (2008): Scale dependency in the species-area relationship of plant communities. - Folia Geobotanica 43: 305-318.

Drakare, S., Lennon, J. J., Hillebrand, H. (2006): The imprint of the geographical, evolutionary and ecological context on species-area relationships. - Ecology Letters 9: 215-227.

Du Rietz, G. E. (1922): Über das Wachsen der Anzahl der konstanten Arten und der totalen Artenzahl mit steigendem Areal in natürlichen Pflanzenassoziationen. - Botaniska Notiser 1922: 17-36.

Dupré, C., Diekmann, M. (2001): Differences in species richness and life-history traits between grazed and abandoned grasslands in southern Sweden. - Ecography 24: 275-286.

Dupré, C., Wessberg, C., Diekmann, M. (2002): Species richness in decidous forests: Effects of species pools and environmental variables. - Journal of Vegetation Science 13: 505-516.

Dupré, C., Stevens, C. J., Ranke, T., Bleeker, A., Peppler-Lisbach, C., Gowing, D. J. G., Dise, N. B., Dorland, E., Bobbink, R., Diekmann, M. (2010): Changes in species richness and composition in European acidic grasslands over the past 70 years: the contribution of cumulative atmospheric nitrogen deposition. - Global Change Biology 16: $344-357$.

Edwards-Jones, G. (2006): Ecological economics and nature conservation. - In: Groom, M. J., Meffe, G. K., Carroll, C. R. [Eds.]: Principles of Conservation Biology. 3. Aufl.: pp. 137-169. Sinauer, Sunderland, MA.

EEA [European Environment Agency] (2007) [Ed.]: Halting the loss of biodiversity by 2010: proposal for a first set of indicators to monitor progress in Europe . - EEA Technical Report 2007(11): 182 pp. European Environment Agency, Copenhagen.

Eisenberg, M. (2003): Saumgesellschaften NO-Niedersachsens - Soziologie und Pflanzenartenvielfalt. - $112+$ X pp. + 5 Tabellen. Diplomarbeit, Institut für Ökologie und Umweltchemie, Universität Lüneburg.

Eriksson, O. (1993): The species-pool hypothesis and plant community diversity. - Oikos 68: 371-374.

European Commission (2007) [Ed.]: Interpretation Manual of European Union Habitats - EUR27. - 144 pp. European Commission, DG Environment, Brussels.

Ewald, J. (2001): Der Beitrag pflanzensoziologischer Datenbanken zur vegetationsökologischen Forschung. Berichte der Reinhold-Tüxen-Gesellschaft 13: 53-69.

Ewald, J. (2003a): A critique for phytosociology. - Journal of Vegetation Science 14: 291-296.

Ewald, J. (2003b): The calcareous riddle: Why are there so many calciphilous species in the Central European flora? - Folia Geobotanica 38: 357-366.

Fahrig, L. (2003): Effects of habitat fragmentation on biodiversity. - Annual Review of Ecology, Evolution, and Systematics 34: 487-515.

Faith, D. P. (1992): Conservation evaluation and phylogenetic diversity. - Biological Conservation 61: 1-10.

Farnsworth, N. R. (1988): Screening plants for new medicins. - In: Wilson, E. O. (1988) [Ed.]: Biodiversity: pp. 8397. National Academy Press, Washington, DC.

Fattorini, S. (2006): Detecting biodiversity hotspots by species-area relationships: a case study of Mediterranean beetles. - Conservation Biology 20: 1169-1180.

Fattorini, S. (2007): To fit or not to fit? A poorly fitting procedure produces inconsistent results when the speciesarea relationship is used to locate hotspots. - Biodiversity and Conservation 16: 2531-2538. 
Field, R., Hawkins, B. A., Cornell, H. V., Currie, D. J., Diniz-Filho, A. F., Guégan, J.-F., Kaufman, D. M., Kerr, J. T., Mittelbach, G. G., Oberdorff, T., O’Brien, E. M., Turner, J. R. G. (2009): Spatial species-richness gradients across scales: a meta-analysis. - Journal of Biogeography 36: 132-147, Oxford.

Fischer, M., Kalko, E. K. V., Linsenmair, K. E., Pfeiffer, S., Prati, D., Schulze, E.-D., Weisser, W. W. (2010): Exploratories for large-scale and long-term functional biodiversity research. - In: Müller, F., Baessler, C., Schubert, H., Klotz, S. [Eds.]: Long-Term Ecological Research - Between Theory and Application: pp. 429-443. Springer, Berlin.

Fridley, J. D., Peet, R. K., Wentworth, T. R., White, P. S. (2005): Connecting fine- and broad-scale species-area relationships of southeastern U. S. flora. - Ecology 86: 1172-1177.

Friedel, A., Oheimb, G. von, Dengler, J., Härdtle, W. (2006): Species diversity and species composition of epiphytic bryophytes and lichens - a comparison or managed and unmanaged beech forests in NE Germany. - Feddes Repertorium 117: 172-185.

Gaston, K. J., Blackburn, T. M. (2000): Pattern and Process in Macroecology. - XII + 377 pp. Blackwell, Malden, MA.

Gaston, K. J., Spicer, J. I. (2004): Biodiversity: an Introduction. 2. Aufl. - XV + 191 pp. Blackwell, Oxford.

Gaston, K. J., Evans, K. L., Lennon, J. J. (2007): The scaling of spatial turnover: pruning the thicket. - In: Storch, D., Marquet, P. A., Brown, J. H. [Eds.]: Scaling Biodiversity: pp. 181-222. Cambridge University Press, Cambridge.

Gleason, H. A. (1922): On the relation between species and area. - Ecology 3: 158-162.

Gleason, H. A. (1929): The significance of Raunkiaer's law of frequency. - Ecology 10: 406-408.

Gotelli, N. J., Colwell, R. K. (2001): Quantifying biodiversity: procedures and pitfalls in the measurement and comparison of species richness. - Ecology Letters 4: 379-391.

Grace, J. B. (1999): The factors controlling species density in herbaceous plant communities: an assessment. Perspectives in Plant Ecology, Evolution and Systematics 2: 1-28.

Graham, C. H., Fine, P. V. A. (2008): Phylogenetic beta diversity: linking ecological and evolutionary processes across space in time. - Ecology Letters 11: 1265-1277.

Gray, J. S., Ugland, K. I., Lambshead, J. (2004a): Species accumulation and species area curves - a comment on Scheiner (2003). - Global Ecology and Biogeography 13: 469-476.

Gray, J. S., Ugland, K. I., Lambshead, J. (2004b): On species accumulation and species-area curves. - Global Ecology and Biogeography 13: 567-568.

Grime, J. P. (1973): Competitive exclusion in herbaceous vegetation. - Nature 242: 344-347.

Groom, M. J. (2006): Threats to biodiversity. - In: Groom, M. J., Meffe, G. K., Carroll, C. R. [Eds.]: Principles of Conservation Biology. 3. Aufl.: pp. 63-109. Sinauer, Sunderland, MA.

Grubb, P. J. (1977): The maintenance of species-richnes in plant communities: the importance of the regeneration niche. - Biological Reviews of the Cambridge Philosophical Society 52: 107-145.

Gruttke, H. (2005) [,2004“] [Ed.]: Ermittlung der Verantwortlichkeit für die Erhaltung mitteleuropäischer Arten. Naturschutz und Biologische Vielfalt 8: 280 pp. Bundesamt für Naturschutz, Bonn.

Grytnes, J. A., Heegaard, E., Romdal, T. S. (2008): Can the mass effect explain the mid-altitudinal peak in vascular plant species richness? - Basic and Applied Ecology 9: 373-382. 
Guilhaumon, F., Gimenez, O., Gaston, K. J., Mouillot, D. (2008): Taxonomic and regional uncertainty in speciesarea relationships and the identification of richness hotspots. - Proceedings of the National Academy of Sciences of the USA 105: 15458-15463.

Haarmeyer, D. H., Schmiedel, U., Dengler, J., Bösing, B. M. (2010a): How does grazing intensity affect different vegetation types in arid Succulent Karoo, South Africa? Implications for conservation management. - Biological Conservation 143: 588-596.

Haarmeyer, D. H., Luther-Mosebach, J., Dengler, J., Schmiedel, U., Finckh, M., Berger, K., Deckert, J., Domptail, S. E., Dreber, N., Gibreel, T., Grohmann, C., Gröngröft, A., Haensler, A., Hanke, W., Hoffmann, A., Husted, L. B., Kangombe, F. N., Keil, M., Krug, C. B., Labitzky, T., Linke, T., Mager, D., Mey, W., Muche, G., Naumann, C., Pellowski, M., Powrie, L. W., Pröpper, M., Rutherford, M. C., Schneiderat, U., Strohbach, B. J., Vohland, K., Weber, B., Wesuls, D., Wisch, U., Zedda, L., Büdel, B., Darienko, T., Deutschewitz, K., Dojani, S., Erb, E., Falk, T., Friedl, T., Kanzler, S.-E., Limpricht, C., Linsenmair, K. E., Mohr, K., Oliver, T., Petersen, A., Rambold, G., Zeller, U., Austermühle, R., Bausch, J., Bösing, B. M., Classen, N., Dorendorf, J., Dorigo, W., Esler, K. J., Etzold, S., Graiff, A., Grotehusmann, L., Hecht, J., Hoyer, P., Kongor, R. Y., Lang, H., Lieckfeld, L. A. B., Oldeland, J., Peters, J., Röwer, I. U., September, Z. M., Sop, T. K., van Rooyen, M. W., Weber, J., Willer, J., Jürgens, N. (2010b): The BIOTA Observatories. - In: Jürgens, N., Haarmeyer, D. H., Luther-Mosebach, J., Dengler, J., Finckh, M., Schmiedel, U. [Eds.]: Biodiversity in Southern Africa. Volume 1: Patterns at Local Scale - the BIOTA Observatories: pp. 6-801. Klaus Hess Publishers, Göttingen \& Windhoek.

Haddad, N. M., Crutsinger, G. M., Gross, K., Haarstad, J., Tilman, D. (2011): Plant diversity and the stability of foodwebs. - Ecology Letters 14: 42-46.

Hallberg, H. P. (1971): Vegetation auf den Schalenablagerungen in Bohuslän, Schweden. - Acta Phytogeographica Suecica 56: 136 pp. + Anhang. Svenska Växtgeografiska Sällskapet, Uppsala.

Hardy, O. J., Senterre, B. (2007): Characterizing the phylogenetic structure of communities by an additive partitioning of phylogenetic diversity. - Journal of Ecology 95: 493-506.

Harper, J. L., Hawksworth, D. L. (1995): Biodiversity: measurement and estimation - Preface. - Philosophical Transactions of the Royal Society of London, B 345: 5-12.

Harte, J. (2007): Towards a mechanistic vasis for a unified theory of spatial structure in ecological communities at multiple spatial scales - In: Storch, D., Marquet, P. A., Brown, J. H. [Eds.]: Scaling Biodiversity: pp. 101-126. Cambridge University Press, Cambridge.

Harte, J., McCarthy, S., Taylor, K., Kinzig, A., Fischer, M. L. (1999): Estimating species-area relationships from plot to landscape scale using species spatial-turnover data. - Oikos 86: 45-54.

Harte, J., Smith, A. B., Storch, D. (2009): Biodiversity scales from plots to biomes with a universal species-area curve. - Ecology Letters 12: 789-797.

He, F., Legendre, P. (1996): On species-area relations. - American Naturalist 148: 719-737.

Hector, A., Schmid, B., Beierkuhnlein, C., Caldeira, M. C., Diemer, M., Dimitrakopoulos, P. G., Finn, J. A., Freitas, H., Giller, P. S., Good, J., Harris, R., Högberg, P., Huss-Danell, K., Joshi, J., Jumpponen, A., Körner, C., Leadley, P. W., Loreau, M., Minns, A., Mulder, C. P. H., O’Donnovan, G., Otway, S. J., Pereira, J. S., Prinz, A., Read, D. J., Scherer-Lorenzen, M., Schulze, E.-D., Siamantziouras, A.-S. D., Spehn, E. M., Terry, A. C., Troumbis, A. Y., Woodward, F. I., Yachi, S., Lawton, J. H. (1999): Plant diversity and productivity experiments in European grasslands. - Science 286: 1123-1127. 
Helm, A., Hanski, I., Pärtel, M. (2006): Slow response of plant species richness to habitat loss and fragmentation. Ecology Letters 9: 72-77.

Hijmans, R. J., Cameron, S. E., Parra, J. L., Jones, P. G., Jarvis, A. (2005): Very high resolution interpolated climate surfaces for global land areas. - International Journal of Climatology 25:1965-1978.

Hill, M. O. (1973): Diversity and evenness: A unifying notation and its consequences. - Ecology 54: 427-431.

Hill, M. O. (1979): TWINSPAN - A FORTRAN program for arranging multivariate data in an ordered two-way table by classification of the individuals and attributes. $-90 \mathrm{pp}$. Cornell University, Ithaca, NY.

Hobohm, C. (1998): Pflanzensoziologie und die Erforschung der Artenvielfalt - Überarbeitete und erweiterte Fassung der an der Universität Lüneburg eingereichten und angenommenen Habilitationsschrift. - Archiv naturwissenschaftlicher Dissertationen 5: 231 pp. Galunder, Wiehl.

Hooper, D. U., Chapin, F. S. III, Ewel, J. J., Hector, A., Inchausti, P., Lavorel, S., Lawton J. H., Lodge, D. M., Loreau, M., Naeem, S., Schmid, B., Setälä, H., Symstad, A. J., Vandermeer, J., Wardle, D. A. (2005): Effects of biodiversity on ecosystem functioning: a consensus on current knowledge. - Ecological Monographs 75: 3-35.

Hubbell, S. P. (2001): The Unified Neutral Theory of Biodiversity and Biogeography. - Monographs in Population Biology 32: XIV + 375 pp. Princeton University Press, Princeton.

Hunter, M. L., Jr. (2002): Fundamentals of Conservation Biology. 2. Aufl. - XVI + 547 pp., Blackwell, Malden, MA.

Illyés, E., Chytrý, M., Botta-Dukát, Z., Jandt, U., Škodová, I., Janišova, M., Willner, W., Hájek, O. (2007): Semidry grasslands along a climatic gradient across Central Europe: Vegetation classification with validation. Journal of Vegetation Science 18: 835-846.

Iltis, H. H. (1988): Serendipity in the exploration of biodiversity - What good are weedy tomatoes? - In: Wilson, E. O. (1988) [Ed.]: Biodiversity: pp. 98-105. National Academy Press, Washington, DC.

IUCN [International Union for Conservation of Nature] 2011 [Ed.]: IUCN red list of threatened species. Version 2011.1. - URL: http://www.iucnredlist.org [Zugriff am 22.10.2011].

Jäger, E. J., Werner, K. (1995) [Eds.]: Gefäßpflanzen: Atlasband. - In: Rothmaler, W. [Founder]: Exkursionsflora von Deutschland 3. 9. Aufl. - 753 pp. Fischer, Jena.

Jandt, U., von Wehrden, H., Bruelheide, H. (2011): Exploring large vegetation databases to detect temporal trends in species occurrences. - Journal of Vegetation Science 22: 957-972.

Janišová, M. (2007) [Ed.]: Grassland vegetation of Slovak Republic-Electronic Expert System for Identification of Syntaxa [slowakisch, mit englischer Zusammenfassung]. - 263 pp. + CD-ROM. Botanický ústav SAV, Bratislava.

Jansen, F., Dengler, J. (2008): GermanSL - Eine universelle taxonomische Referenzliste für Vegetationsdatenbanken in Deutschland. - Tuexenia 28: 239-253.

Jansen, F., Dengler, J. (2010): Plant names in vegetation databases - a neglected source of bias. - Journal of Vegetation Science 21: 1179-1186.

Jansen, F., Dengler, J., Glöckler, F., Chytrý, M., Ewald, J., Oldeland, J., Schaminée, J. H. J. (2011): Die mitteleuropäischen Datenbanken im Global Index of Vegetation-Plot Databases (GIVD). - Tuexenia 31: 351367.

Johnson, J. B., Omland, K. S. (2004): Model selection in ecology and evolution. - Trends in Ecology \& Evolution 19: $101-108$. 
Jonsell, B., Karlsson, T. (2004): Endemic vascular plants in Norden. - In: Jonsell, B. [Ed.]: Flora Nordica General Volume: pp. 139-159. Royal Swedish Acad. of Sciences, Stockholm.

Jost, L. (2007): Partitioning diversity into independent alpha and beta components. - Ecology 88: 2427-2439.

Jurasinksi, G., Retzer, V., Beierkuhnlein, C. (2009): Inventory, differentiation, and proportional diversity: a consistent terminology for quantifying species diversity. - Oecologia 159: 15-26.

Jürgens, N., Schmiedel, U., Haarmeyer, D. H., Dengler, J., Finckh, M., Goetze, D., Gröngröft, A., Hahn, K., Koulibaly, A., Luther-Mosebach, J., Muche, G., Oldeland, J., Petersen, A., Porembski, S., Rutherford, M. C., Schmidt, M., Sinsin, B., Strohbach, B. J., Thiombiano, A., Wittig, R., Zizka, G. (im Druck): The BIOTA Biodiversity Observatories in Africa - A standardized framework for large-scale environmental monitoring. Environmental Monitoring and Assessment. DOI: 10.1007/s10661-011-1993-y.

Kallimanis, A. S., Halley, J. M., Vokou, D., Sgardelis, S. P. (2008): The scale of analysis determines the spatial pattern of woody species diversity in the Mediterranean environment. - Plant Ecology 196: 143-151.

Kareiva, P. (2001): Ecoinformtics: facilitating access to existing data sets. - Trends in Ecology \& Evolution 16: 226.

Kaufman, D. M. (1995): Diversity of New World mammals: universality of the latitudinal gradients of species and bauplans. - Journal of Mammology 76: 322-334.

Kim, E.-S. (2006). Development, potentials, and challenges of the International Long-Term Ecological Research (ILTER) Network. - Ecological Research 21: 788-793.

Klein, A.-M., Müller, C., Hoehn, P., Kremen, C. (2009): Understanding the role of species richness for crop pollination services. - In: Naeem, S., Bunker, D. E., Hector, A., Loreau, M., Perrings, C. (2009) [Eds.]: Biodiversity, Ecosystem Functioning, and Human Wellbeing - An Ecological and Economic Perspective: pp. 195-208. Oxford University Press, Oxford.

Kliment, J., Jarolímek, I., Šibík, J. (2007): Mulgedio-Aconitetea Hadač et Klika in Klika 1948 [slowakisch, mit englischer Zusammenfassung]. - In: Kliment, J., Valachovič, M. [Eds.]: Plant Communities of Slovakia. 4. High-Mountain Vegetation: Mulgedio-Aconitetea, Betulo carpaticae-Alnetea viridis, Elyno-Seslerietea, Carici rupestris-Kobresietea bellardii, Salicetea herbaceae, Loiseleurio-Vaccinietea, Caricetea curvulae, Nardetea strictae: pp. 21-129. Veda Publisher House Slovak Akademy of Sciences, Bratislava.

Koči, M. (2007): Subalpínská vysokobylinná a křovinná vegetace (Mulgedio-Aconitetea) - Subalpine tall-forb and deciduous-shrub vegetation [tschechisch, mit englische Zusammenfassung]. - In: Chytrý, M. [Ed.]: Vegetation of the Czech Republic - 1. Grassland and Heathland Vegetation: pp. 91-131. Academia, Praha.

Kočí, M., Chytrý, M., Tichý, L. (2003): Formalized reproduction of an expert-based phytosociolocical classification: A case study of subalpine tall-forb vegetation. - Journal of Vegetation Science 14: 601-610.

Koh, L. P., Ghazoul, J. (2010): A matrix-calibrated species-area model for predicting biodiversity losses due to landuse change. - Conservation Biology 24: 995-1001.

Koperski, M., Sauer, M., Braun, W., Gradstein, S. R. (2000): Referenzliste der Moose Deutschlands. Schriftenreihe für Vegetationskunde 34: 519 pp. Bundesamt für Naturschutz, Bonn.

Köppel, C. (2002) [,„2000“]: Die Roten Listen der Pflanzengesellschaften in Europa - eine Literatur-Synopse. - In: Rennwald, E. [Ed.]: Verzeichnis und Rote Liste der Pflanzengesellschaften Deutschlands - mit Datenservice auf CD-ROM. - Schriftenreihe für Vegetationskunde 35: 83-87. Bundesamt für Naturschutz, Bonn.

Krahulec, F., Rosén, E., van der Maarel, E. (1986): Preliminary classification and ecology of dry grassland communities on Ölands Stora Alvar (Sweden). - Nordic Journal of Botany 6: 797-809. 
Krebs, J. (2003): Vegetation und Naturschutz von Wald- und Gebüschsäumen in der Umgebung von Lüneburg. $102+8$ pp. + Beilagemappe. Diplomarbeit, Institut für Ökologie und Umweltchemie, Universität Lüneburg.

Kreft, H., Jetz, W., Mutke, J., Kier, G., Barthlott, W. (2008): Global diversity of island floras from a macroecological perspective. - Ecology Letters 11: 116-127.

Kunin, W. E. (1997): Sample shape, spatial scale and species counts: implications for reserve design. - Biological Conservation 82: 369-377.

Lawton, J. H. (1999): Are there general laws in ecology? - Oikos 84: 177-192.

Lennon, J. J., Kunin, W. E., Hartley, S., Gaston, K. J. (2007): Species distribution patterns, diversity scaling and testing of fractals in southern African birds - In: Storch, D., Marquet, P. A., Brown, J. H. [Eds.]: Scaling Biodiversity: pp. 51-76. Cambridge University Press, Cambridge.

Lenoir, J., Gégout, J. C., Marquet, P. A., Ruffray, P. de, Brisse, H. (2008): A significant upward shift in plant species optimum elevation during the 20th century. - Science 320: 1768-1771.

Löbel, S. (2002): Trockenrasen auf Öland: Syntaxonomie - Ökologie - Biodiversität. - $178+$ XIV pp. + 4 Tabellen. Diplomarbeit, Institut für Ökologie und Umweltchemie, Universität Lüneburg.

Löbel, S., Dengler, J. (2008) [„,2007’]: Dry grassland communities on southern Öland: phytosociology, ecology, and diversity. - In: van der Maarel, E. [Ed.]: Structure and dynamics of alvar vegetation on Öland and some related dry grasslands - Dedicated to Ejvind Rosén on his $65^{\text {th }}$ birthday. - Acta Phytogeographica Suecica 88: 13-32. Svenska Växtgeografiska Sällskapet, Uppsala.

Löbel, S., Dengler, J., Hobohm, C. (2004): Beziehungen zwischen der Artenvielfalt von Gefäßpflanzen, Moosen und Flechten in Trockenrasen der Insel Öland (Schweden). - Kieler Notizen zur Pflanzenkunde SchleswigHolstein und Hamburg 32: 9-13.

Löbel, S., Dengler, J., Hobohm, C. (2006): Species richness of vascular plants, bryophytes and lichens in dry grasslands: The effects of environment, landscape structure and competition. - Folia Geobotanica 41: 377-393.

Loehle, C. (1990): Proper statistical treatment of species-area data. - Oikos 57: 143-145, Oxford.

Lomolino, M. V. (2000): Ecology’s most general, yet protean pattern: the species-area relationship. - Journal of Biogeography 27: 17-26.

Lomolino, M. V. (2001): The species-area relationship: new challenges for an old pattern. - Progress in Physical Geography 25: 1-21, London [u. a.].

Lomolino, M. V., Weiser, M. D. (2001): Towards a more general species-area relationship: diversity on all islands, great and small. - Journal of Biogeography 28: 431-445.

Lomolino, M. V., Riddle, B. R., Whittaker, R. J., Brown, J. H. (2010): Biogeography. 4. Aufl. - XIV + 878 pp. Sinauer, Sunderland, MA.

Loreau, M. (2000): Are communities saturated? On the relationship between $\alpha, \beta$ and $\gamma$ diversity. - Ecology Letters 3: $73-76$.

Loreau, M., Naeem, S., Inchausti, P., Bengtsson, J., Grimpe, J. P., Hector, A., Hooper, D. U., Huston, M. A., Raffaelli, D., Schmid, B., Tilman, D., Wardle, D. A. (2001): Biodiversity and ecosystem functioning: current knowledge and future challenges. - Science 294: 804-808C.

Ludwig, G., Haupt, H., Gruttke, H., Binot-Hafke, M. (2005): Methodische Weiterentwicklung der Roten Listen gefährdeter Tiere, Pflanzen und Pilze in Deutschland - eine Übersicht. - Natur und Landschaft 80: $257-265$.

Lundholm, J. T. (2009): Plant species diversity and environmental heterogeneity: spatial scale and competing hypotheses. - Journal of Vegetation Science 20: 377-391. 
Luther-Mosebach, J., Dengler, J., Schmiedel, U., Röwer, I. U., Labitzky, T., Gröngröft, A. (im Druck): A first formal classification of Hardeveld vegetation in Namaqualand, South Africa. - Applied Vegetation Science. DOI: 10.1111/j.1654-109X.2011.01173.X.

Mace, G. M., Collar, N. J., Gaston, K. J., Hilton-Taylor, C., Akçakaya, H. R., Leader-Williams, N., Milner-Gulland, E. J., Stuart, S. N. (2008): Quantification of extinction risk: IUCN's system of classifying threatened species. Conservation Biology 22: 1424-1442..

Mace, G. M., Cramer, W., Díaz, S., Faith, D. P., Larigauderie, A., Le Prestre, P., Palmer, M., Perrings, C., Scholes, R. J., Walpole, M., Walther, B. A., Watson, J. E. M., Mooney, H. A. (2010): Biodiversity targets after 2010. Current Opinion in Environmental Sustainability 2: 3-8.

Magurran, A. E. (2004): Measuring Biological Diversity. - VIII + 256 pp. Blackwell, Malden, MA.

Magurran, A. E. (2007): Species abundance distributions over time. - Ecology Letters 10: 347-354.

Marshall, L. G. (1988): Extinction. - In: Myers, A. A., Giller, P. S. [Eds.]: Analytical Biogeography - an Integrated Approach to the Study of Animal and Plant Distributions: pp. 219-254. Chapman \& Hall, London.

May, R. M. (1994): Conceptual aspects of the quantification of the extent of biological diversity. - Philosophical Transactions of the Royal Society of London, B 345: 13-20, London.

May, R. M. (2010): Tropical arthropod species, more or less? - Science 329: 41-42.

McGlinn, D. J., Palmer, M. W. (2009): Modeling the sampling effect in the species-time-area relationship. Ecology 90: 836-846.

Michl, T., Dengler, J., Huck, S. (2010): Montane-subalpine tall-herb vegetation (Mulgedio-Aconitetea) in central Europe: large-scale synthesis and comparison with northern Europe. - Phytocoenologia 40: 117-154.

Mooney, H. A., Lubchenco, J., Dirzo, R., Sala, O. E. (1995) [Eds.]: Biodiversity and ecosystem functioning: basic principles. - In: Heywood, V. H., Watson, R. T. [Eds.]: Global Biodiversity Assessment: pp. $275-325$. Cambridge University Press, Cambridge.

Mora, C., Tittensor, D. P., Adl, S., Simpson, A. G. B., Worm, B. (2011): How many species are there on Earth and in the ocean? - PLoS Biology 9(8): e1001127. DOI: 10.1371/journal.pbio.1001127.

Moravec, J. (1973): The determination of the minimal area of phytocoenoses. - Folia Geobotanica \& Phytotaxonomica 8: 23-47.

Mucina, L., Rutherford, M. C. (2006) [Eds.]: The vegetation of South Africa, Lesotho and Swaziland. - Strelitzia 19: 807 pp. + CD-ROM. South African National Biodiversity Institute, Pretoria.

Mucina, L., Rutherford, M. C., Powrie, L. W. (2006): The logic of the map: approaches and procedures. - In: Mucina, L., Rutherford, M. C. [Eds.]: The vegetation of South Africa, Lesotho and Swaziland. - Strelitzia 19: 12-29. South African National Biodiversity Institute, Pretoria.

Myers, N., Mittermeier, R. A., Mittermeier, C. G., Fonseca, G. A. B. de, Kent, J. (2000): Biodiversity hotspots for conservation priorities. - Nature 403: 853-858.

Naeem, S., Thompson, L. J., Lawler, S. P., Lawton, J. H., Woodfin, R. M. (1994): Declining biodiversity can alter the performance of ecosystems. - Nature 368: 734-737.

Naeem, S., Bunker, D. E., Hector, A., Loreau, M., Perrings, C. (2009) [Eds.]: Biodiversity, Ecosystem Functioning, and Human Wellbeing - An Ecological and Economic Perspective. - XIV + 368 pp. Oxford University Press, Oxford.

Nekola, J. C., Brown, J. H. (2007): The wealth of species: ecological communities, complex systems and the legacy of Frank Preston. - Ecology Letters 10: 188-196. 
Nekola, J. C., White, P. S. (1999): The distance decay of similarity in biogeography and ecology. - Journal of Biogeography 26: 867-878.

Nosek, J. N. (1986): Spatial processes in a grassland community, III. Species-area relation, dominance-diversity durves, changes in the frequency and density of the species. - Acta Botanica Hungarica 32: 61-78.

Noss, R. F. (1990): Indicators for monitoring biodiversity: a hierarchical approach. - Conservation Biology 4: 355364.

Olszewski, T. D. (2004): A unified mathematical framework for the measurement of richness and evenness within and among multiple communities. - Oikos 104: 377-387.

Otýpková, Z., Chytrý, M. (2006): Effects of plot size on the ordination of vegetation samples. - Journal of Vegetation Science 17: 465-472.

Palmer, M. W. (1994): Variation in species richenss: towards a unification of hypotheses. - Folia Geobotanica \& Phytotaxonomica 29:511-530.

Palmer, M. W. (2007): Species-area curves and the geometry of nature. - In: Storch, D., Marquet, P. A., Brown, J. H. [Eds.]: Scaling Biodiversity: pp. 15-31. Cambridge University Press, Cambridge.

Palmer, M. W., White, P. S. (1994): On the existence of ecological communities. - Journal of Vegetation Science 5: 279-282.

Pärtel, M. (2002): Local plant diversity patterns and evolutionary history at the regional scale. - Ecology 83: 23612366.

Pärtel, M., Kalamees, R., Zobel, M., Rosén, E. (1999): Alvar grasslands in Estonia: variation in species composition and community structure. - Journal of Vegetation Science 10: 561-568.

Pausas, J. G., Austin, M. P. (2001): Patterns of plant species richness in relation to different environments: An appraisal. - Journal of Vegetation Science 12: 153-166.

Pereira, H. M., Leadley, P. W., Proenca, V., Alkemade, R., Scharlemann, J, P. W., Fernandez-Manjarrés, J. F., Araújo, M. B., Balvanera, P., Biggs, R., Cheung, W. W. L., Chini, L., Cooper, H. D., Gilman, E. L., Guénette, S., Hurtt, G. C., Huntington, H. P., Mace, G. M., Oberdorff, T., Revenga, C., Rodrigues, P., Scholes, R. J., Sumaila, U. R., Waldpole, M. (2010): Scenarios for global biodiversity in the 21st century. - Science 330: 14961501.

Petchey, O. L., Gaston, K. J. (2002): Functional diversity (FD), species richness and community composition. Ecology Letters 5: 402-411.

Petchey, O. L., Gaston, K. J. (2006): Functional diversity: back to the basics and looking forward. - Ecology Letters 9: 741-758.

Peters, J. (2010): Plant diversity patterns at different spatial scales in a semi-arid savanna ecosystem in central Namibia. - 64 + IX pp. + CD-ROM. Diplom thesis in Landscape Ecology \& Nature Conservation, Ernst-MoritzArndt University, Greifswald. URL: http://www.biologie.unihamburg.de/bzf/syst/Diplom_thesis_Jan_Peters_2009.pdf.

Petersen, A. (2008): Pedodiversity of southern African drylands. - Hamburger Bodenkundliche Arbeiten 61: 375 pp. Verein zur Förderung der Bodenkunde in Hamburg, Hamburg.

Peterson, D. L., Parker, V. T. (1998) [Eds.]: Ecological Scale - Theory and Applications. - XVII + 615 pp. Columbia University Press, New York.

Pimm, S. L., Russell, G. J., Gittleman, J. L., Brooks, T. M. (1995): The future of biodiversity. - Science 269: 347350 . 
Plotkin, J. B., Potts, M. D., Yu, D. W., Bunyavejchewin, S., Condit, R., Foster, R., Hubbell, S. P., LaFrankie, J., Manokaran, N., Seng, L. H., Sukumar, R., Nowak, M. A., Ashton, P. S. (2000): Predicting species diversity in tropical forests. - Proceedings of the National Academy of Sciences of the USA 97: 10850-10854.

Plotkin, M. J. (1988): The outlook for new agricultural and industrial products from the tropics - In: Wilson, E. O. (1988) [Ed.]: Biodiversity: pp. 106-116. National Academy Press, Washington, DC.

Pompe, S., Hanspach, J., Badeck, F., Klotz, S., Thuiller, W., Kühn, I. (2008): Climate and land use change impacts on plant distributions in Germany. - Biology Letters 4: 564-567.

Preston, F. W. (1962): The canonical distribution of commonness and rarity: Part II. - Ecology 43: 410-432.

Purvis, A., Hector, A. (2000): Getting the measure of biodiversity. - Nature 405: 212-219, London.

Qian, H., Ricklefs, R. E. (2007): A latitudinal gradient in large-scale beta diversity for vascular plants in North America. - Ecology Letters 10: 737-744.

Qian, H., Ricklefs, R. E. (2008): Global concordance in diversity patterns of vascular plants and terrestrial vertebrates. - Ecology Letters 11: 547-553.

Qian, H., Ricklefs, R. E., White, P. S. (2005): Beta diversity of angiosperms in temperate floras of eastern Asia and eastern North America. - Ecology Letters 8: 15-22.

Qiu, Y.-L., Li, L., Wang, B., Chen, Z., Knoop, V., Groth-Malonek, M., Dombrovska, O., Lee, J., Kent, L., Rest, J., Estabrook, G. F., hendry, T. A., Taylor, D. W., Testa, C. M., Ambros, M., Crandall-Stotler, B., Duff, R. J., Stech, M., Frey, W., Quandt, D., Davis, C. C. (2006): The deepest divergences in land plants inferred from phylogenomic evidence. - Proceedings of the National Academy of Sciences of the USA 103: 15511-15516.

Quinn, G. P., Keough, M. J. (2002): Experimental Design and Data Analysis for Biologists. - XVII + 537 pp. Cambridge University Press, Cambridge.

Rands, M. R. W., Adams, W. M., Bennun, L., Butchart, S. H. M., Clements, A., Coomes, D., Entwistle, A., Hodge, I., Kapos, V., Scharlemann, J. P. W., Sutherland, W. J., Vira, B. (2010): Biodiversity conservation: challenges beyond 2010. - Science 329: 1298-1303.

Raup, D. M. (1994): The role of extinction in evolution. - Proceedings of the National Academy of Sciences of the USA 91: 6758-6763.

Raup, D. M., Sepkoski, J. J. Jr. (1982): Mass extinctions in the marine fossil record. - Science 215: 1501-1503.

Reed, R. A., Peet, R. K., Palmer, M. W., White, P. S. (1993): Scale dependence of vegetation-environment correlations: A case study of a North Carolina piedmont woodland. - Journal of Vegetation Science 4: 329-340.

Rennwald, E. (2002) [,,2000“] [Ed..]: Verzeichnis und Rote Liste der Pflanzengesellschaften Deutschlands - mit Datenservice auf CD-ROM. - Schriftenreihe für Vegetationskunde 35: 800 pp. + CD-ROM. Bundesamt für Naturschutz, Bonn.

Ricklefs, R. E. (2004): A comprehensive framework for global patterns in biodiversity. - Ecology Letters 7: 1-15.

Ricklefs, R. E., Schluter, D. (1993) [Eds.]: Species Diversity in Ecological Communities - Historical and Geographical Perspectives. - 416 pp. University of Chicago Press, Chicago.

Ricotta, C. (2005a): On hierarchical diversity decomposition. - Journal of Vegetation Science 16: 223-226.

Ricotta, C. (2005b): A note on functional diversity measures. - Basic and Applied Ecology 6: 479-486.

Ritchie, M. E. (2010): Scale, heterogeneity, and the structure of ecological communities. - In: Levin, S. A., Horn, H. S. [Eds.]: Monographs in Population Biology 45: 229 pp. Princeton University Press, Princeton.

Ritchie, M. E., Olff, H. (1999): Spatial scaling laws yield a synthetic theory of biodiversity. - Nature 400: 557-560. 
Rodwell, J. S. (1991) [Ed.]: British Plant Communities. Volume $1-$ Woodland and Scrub. $-\mathrm{X}+395$ pp. Cambridge University Press, Cambridge.

Rodwell, J. S., Schaminée, J. H. J., Mucina, L., Pignatti, S., Dring, J., Moss, D. (2002): The diversity of European vegetation - An overview of phytosociological alliances and their relationships to EUNIS habitats. - Rapport EC-LNV 2002/054: 168 pp. National Reference Centre for Agriculture, Nature and Fisheries, Wageningen.

Rosenzweig, M. L. (1995): Species Diversity in Space and Time. - XXI + 436 pp., Cambridge University Press, Cambridge.

Ruprecht, E., Szabó, A., Enyedi, M. Z., Dengler, J. (2009): Steppe-like grasslands in Transylvania (Romania): characterisation and influence of management on species diversity and composition. - Tuexenia 29: 353-368 + 1 Tabelle.

Sala, O. E., Chapin, F. S., III, Armesto, J. J., Berlow, E., Bloomfield, J., Dirzo, R., Huber-Sanwald, E., Huenneke, L. F., Jackson, R. B., Kinzig, A., Leemans, R., Lodge, D. M., Mooney, H. A., Oesterheld, M., LeRoy Poff, N., Sykes, M. T., Walker, B. H., Walker, M., Wall, D. H. (2000): Global biodiversity scenarios for the year 2100. Science 287: 1770-1774.

Schaminée, J. H. J., Stortelder, A. H. F., Weesthoff, V. (1995) [Eds.]: De Vegetatie von Nederland - Deel 1. Inleiding tot de Plantensociologie - Grondslagen, Methoden en Toepassingen [niederländisch]. - 296 pp. Opulus, Uppsala.

Schaminée, J. H. J., Hennekens, S. M., Chytrý, M., Rodwell, J. S. (2009): Vegetation-plot data and databases in Europe: an overview. - Preslia 81: 173-185.

Scheiner, S. M. (2003): Six types of species-area curves. - Global Ecology and Biogeography 12: 441-447.

Scheiner, S. M. (2004): A mélange of curves - further dialogue about species-area relationships. - Global Ecology and Biogeography 13: 479-484.

Scheiner, S. M. (2009): The terminology and use of species-area relationships: a response to Dengler (2009). Journal of Biogeography 36: 2005-2008.

Scheiner, S. M., Cox, S. B., Willig, M., Mittelbach, G. G., Osenberg, C., Kaspari, M. (2000): Species richness, species-area curves and Simpson's paradox. - Evolutionary Ecology Research 2: 791-802.

Scheiner, S. M., Chiarucci, A., Fox, G. A., Helmus, M. R., McGlinn, D., Willig, M. R. (2011): The underpinnings of the relationship of species richness with space and time. - Ecological Monographs 81: 195-213.

Schmiedel, U., Dengler, J., Luther-Mosebach, J., Gröngröft, A., Muche, G., Petersen, A., Strohbach, B. J., Jürgens, N. (2010): Patterns and dynamics of vascular plant diversity along the BIOTA transects in southern Africa. - In: Schmiedel, U., Jürgens, N. [Eds.]: Biodiversity in Southern Africa. Volume 2: Patterns and Processes at Regional Scale: pp. 118-135. Klaus Hess Publishers, Göttingen \& Windhoek.

Schmiedel, U., Dengler, J., Etzold, S. (im Druck): Vegetation dynamics of endemic-rich quartz fields in the Succulent Karoo, South Africa, in response to recent climatic trends. - Journal of Vegetation Science. DOI: 10.1111/j.1654-1103.2011.01346.x.

Schnittler, M., Ludwig, G. (1996): Zur Methodik der Erstellung Roter Listen. - In: Ludwig, G., Schnittler, M. [Eds.]: Rote Listen gefährdeter Pflanzen Deutschlands. - Schriftenreihe für Vegetationskunde 28: 709-739. Bundesamt für Naturschutz, Bonn.

Schoener, T. W. (1976): The species-area relation within archipelagos: models and evidence from island land birds. - Proceedings of the International Ornithological Congress 16: 628-642. 
Scholes, R. J., Mace, G. M., Turner, W., Geller, G. N., Jürgens, N., Larigaudrie, A., Muchoney, D., Walther, B. A., Mooney, H. A. (2008): Toward a global biodiversity observing system. - Science 321: 1044-1045.

Schuster, B., Diekmann, M. (2003): Changes in species density along the soil pH gradient - evidence from German plant communities. - Folia Geobotanica 38: 367-379.

Schutyser, F., Condé, S., Hoogeveen, Y. (2009): Progress towards the European 2010 biodiversity target - indicator fact sheets. Compendium to EEA Report No 4/2009. - EEA Technical Report 2009(5): 78 pp. European Environmental Agency, Copenhagen.

Schweiger, O., Klotz, S., Durka, W., Kühn, I. (2008): A comparative test of phylogenetic diversity indices. Oecologia 157: 485-495.

Secretariat of the Convention on Biological Diversity (2010): Global Biodiversity Outlook 3. - 94 pp. Secretariat of the Convention on Biological Diversity, Montreal.

Sfenthourakis, S., Triantis, K. A. (2009): Habitat diversity, ecological requirements of species and the Small Island Effect. - Diversity and Distributions 15: 131-140, Oxford.

Shmida, A., Wilson, M. V. (1985): Biological determinants of species diversity. - Journal of Biogeography 12: 120.

Sitte, P., Weiler, E. W., Kadereit, J. W., Bresinsky, A., Körner, C. (2002): Lehrbuch der Botanik für Hochschulen.In: Strasburger, E., Noll, F., Schenck, H., Schimper, A. F. W. [Founder.]: 35. Aufl. XIV + 1123 pp. Spektrum Akademischer Verlag, Heidelberg.

Šizling, A. L., Storch, D. (2004): Power-law species-area relationships and self-similar species distributions within finite areas. - Ecology Letters 7: 60-68.

Sjögren, E. (1988) [Ed.]: Plant cover on the limestone Alvar of Öland: ecology - sociology - taxonomy. - Acta Phytogeographica Suecica 76: 160 pp. Svenska Växtgeografiska Sällskapet, Uppsala.

Škornik, S., Vidrih, M., Kaligarič, M. (2010): The effect of grazing pressure on species richness, composition and productivity in North Adriatic Karst pastures. - Plant Biosystems 144: 355-364.

Smith, B., Wilson, J. B. (1996): A consumer's guide to evenness indices. - Oikos 76: 70-82.

Stark, S. C., Bunker, D. E., Carson, W. P. (2006): A null model of exotic plant diversity tested with exotic and native species-area relationships. - Ecology Letters 9: 136-141.

Stiles, A., Scheiner, S. M. (2007): Evaluation of species-area functions using Sonoran Desert plant data: not all species-area curves are power functions. - Oikos 116: 1930-1940.

Stohlgren, T. J. (2007): Measuring Plant Diversity - Lessons from the Field. - XVII +390 pp. Oxford University Press, Oxford.

Stohlgren, T. J., Falkner, M. B., Schell, L. D. (1995): A Modified-Whittaker nested vegetation sampling method. Vegetatio 117: 113-121.

Storch, D., Gaston, K. J. (2004): Untangling ecological complexity on different scales of space and time. - Basic and Applied Ecology 5: 389-400.

Storch, D., Šizling, A. L. (2008): The concept of taxon invariance in ecology: do diversity patterns vary with changes in taxonomic resolution? - Folia Geobotanica 43: 329-344.

Storch, D., Marquet, P. A., Brown, J. H. (2007a) [Eds.]: Scaling Biodiversity. - XVII + 470 pp. Cambridge University Press, Cambridge. 
Storch, D., Marquet, P. A., Brown, J. H. (2007b): Introduction: scaling biodiversity - what is the problem? - In: Storch, D., Marquet, P. A., Brown, J. H. [Eds.]: Scaling Biodiversity: pp. 1-11. Cambridge University Press, Cambridge.

Storch, D., Šizling, A. L., Reif, J., Polechová, J., Šizlingová, E., Gaston, K. J. (2008): The quest for a null model for macroecological patterns: geometry of species distributions at multiple spatial scales. - Ecology Letters 11: 771-784.

Thomas, C. D., Cameron, A., Green, R. E., Bakkenes, M., Beaumont, L. J., Collingham, Y. C., Erasmus, B. F. N., Siqueira, M. F. de, Grainger, A., Hannah, L., Hughes, L., Huntley, B., Jarsveld, A. S. van, Midgley, G. F., Miles, L., Ortega-Huerta, M. A., Peterson, A. T., Phillips, O. L., Williamson, S. E. (2004): Extinction risk from climate change. - Nature 427: 145-147.

Tichý, L. (2002): JUICE, software for vegetation classification. - Journal of Vegetation Science 13: 451-453.

Tichý, L. (2005): New similarity indices for the assignment of relevés to the vegetation units of an existing phytosociological classification. - Plant Ecology 179: 67-72.

Tichý, L., Chytrý, M. (2006): Statistical determination of diagnostic species for site groups of unequal size. Journal of Vegetation Science 17: 809-818.

Tichý, L., Chytrý, M., Hájek, M., Talbot, S. S., Botta-Dukát, Z. (2010): OptimClass: Using species-to-cluster fidelity to determine the optimal partition in classification of ecological communities. - Journal of Vegetation Science 21: 287-299.

Tilman, D. (1994): Competition and biodiversity in spatially structured habitats. - Ecology 75: 2-16.

Tilman, D. (2000): Causes, consequences and ethics of biodiversity. - Nature 405: 208-211.

Tilman, D., Wedin, D., Knops, J. (1996): Productivity and sustainability influenced by biodiversity in grassland ecosystems. - Nature 379: 718-720.

Timmermann, T., Dengler, J., Abdank, A., Berg, C. (2006): Objektivierung von Naturschutzbewertungen - Das Beispiel Roter Listen von Pflanzengesellschaften. - Naturschutz und Landschaftsplanung 38: 133-139.

Tittensor, D. P., Micheli, F., Nyström, M., Worm, B. (2007): Human impacts on the species-area relationship in reef fish assemblages. - Ecology Letters 10: 760-772.

Tjørve, E. (2003): Shapes and functions of species-area curves: a review of possible models. - Journal of Biogeography 30: 827-835.

Tjørve, E. (2009): Shapes and functions of species-area curves (II): a review of new models and parameterizations. Journal of Biogeography 36: 1435-1445.

Tjørve, E., Tjørve, K. M. C. (2011): Subjecting the theory of the small-island effect to Ockham's razor. - Journal of Ecology 96: 1141-1151.

Tonné, F. (1954): Besser bauen mit Besonnungs- und Tageslicht-Planung. - 41 pp. Hoffmann, Schorndorf.

Tremp, H. (2005): Aufnahme und Analyse vegetationsökologischer Daten. - UTB 8299: 141 pp. Ulmer, Stuttgart.

Tsiripidis, I., Bergmeier, E., Fotiadis, G., Dimopoulos, P. (2009): A new algorithm for the determination of differential taxa. - Journal of Vegetation Science 20: 233-240.

Tuomisto, H. (2010a): A diversity of beta diversities: straightening up a concept gone awry. Part 1. Defining beta diversity as a function of alpha and gamma diversity. - Ecography 33: 2-22.

Tuomisto, H. (2010b): A diversity of beta diversities: straightening up a concept gone awry. Part 2. Quantifying beta diversity and related phenomena. - Ecography 33: 23-45.

Turner, W. R., Tjørve, E. (2005): Scale-dependence in species-area relationships. - Ecography 28: 721-730. 
Turner, W. R., Brandon, K., Brooks, T. M., Costanza, R., da Fonseca, G. A. B., Portela, R. (2007): Global conservation of biodiversity and ecosystem services. - BioScience 57: 868-873.

Turtureanu, P. D., Dengler, J. (im Druck): Different aspects of plant diversity show contrasting patterns in Carpathian forest openings. - Plant Ecology. DOI: 10.1007/s11258-011-0007-z.

Ulrich, W. (2005): Predicting species numbers using species-area and endemic-area relations. - Biodiversity and Conservation 14: 3351-3362.

United Nations (1992): Convention on Biological Diversity. - URL: http://www.cbd.int/doc/legal/cbd-en.pdf [Zugriff am 16.10.2011].

Valachovič, M. (1995) [Ed.]: Rastlinné spoločenstvá Slovenska. 1. Pionierska vegetácia: Asplenietea trichomanis, Thlaspietea rotundifolii, Sedo-Scleranthetea, Koelerio-Corynephoretea, Festuceta vaginatae, Lemnetea, Potametea [slowakisch]. - 184 pp. Veda Vydavastel'stvo Slovenskej Akadémie Vied, Bratislava.

van der Maarel, E. (1997): Biodiversity: from bable to biosphere management. - Special Features in Biosystematics and Biodiversity 2: 60 pp. Opulus, Uppsala.

van der Maarel, E. (2008) [,,2007”] [Ed.]: Structure and dynamics of alvar vegetation on Öland and some related dry grasslands - Dedicated to Ejvind Rosén on his 65 ${ }^{\text {th }}$ birthday. - Acta Phytogeographica Suecica 88: 98 pp. Svenska Växtgeografiska Sällskapet, Uppsala.

Vassilev, K., Pedashenko, H., Nikolov, S. C., Apostolova, I., Dengler, J. (2011): Effect of land abandonment on the vegetation of upland semi-natural grasslands in the Western Balkan Mts., Bulgaria. - Plant Biosystems 145: $654-665$.

Veech, J. A. (2000): Choice of species-area function affects identification of hotspots. - Conservation Biology 14: $140-147$.

Vellend, M. (2001): Do commonly used indices of $\beta$-diversity measure species turnover? - Journal of Vegetation Science 12: 545-552.

Villéger, S., Mason, N. W. H., Mouillot, D. (2008): New multidimensional functional diversity indices for a multifaceted framework in functional ecology. - Ecology 89: 2290-2301.

Vitousek, P. M., Mooney, H. A., Lubchenco, J., Melillo, J. M. (1997): Human domination of Earth's ecosystems. Science 277: 494-499.

Wallenfang, J. (2011): Testing the existence of the small island effect. $-41+$ XXVIII pp. + CD-ROM. Bachelorarbeit in Biologie, Univ. Hamburg.

Weibull, A.-C., Östman, Ö., Granqvist, Å. (2003): Species richness in agroecosystems: the effect of landscape, habitat and farm management. - Biodiversity and Conservation 12: 1335-1355.

Westhoff, V., van der Maarel, E. (1973): The Braun-Blanquet-Approach. - Whittaker, R. H. [Ed.]: Ordination and classification of communities. - Handbook of Vegetation Science 5: pp. 617-726. Junk, The Hague.

White, E. P. (2007): Spatiotemporal scaling of species richness: patterns, processes, and implications. - In: Storch, D., Marquet, P. A., Brown, J. H. [Eds.]: Scaling Biodiversity: pp. 325-346. Cambridge University Press, Cambridge.

Whittaker, R. H. (1960): Vegetation of the Siskiyou Mountains, Oregon and California. - Ecological Monographs 30: 279-338.

Whittaker, R. H. (1972): Evolution and measurement of species diversity. - Taxon 21: 213-251.

Whittaker, R. J., Fernández-Palacios, J. M. (2007): Island Biogeography-Ecology, Evolution, and Conservation. 2. Aufl. - XII + 401 pp. Oxford University Press., Oxford. 
Whittaker, R. J., Willis, K. J., Field, R. (2001): Scale and species richness: towards a general, hierarchical theory of species diversity. - Journal of Biogeography 28: 453-470.

Williams, M. R. (1996): Species-area curves: the need to include zeroes. - Global Ecology and Biogeography Letters 5: 91-93.

Williamson, M. (1988): Relationship of species number to area, distance and other variables. - In: Myers, A. A., Giller, P. S. [Eds.]: Analytical Biogeography: An Integrated Approach to the Study of Animal and Plant Distributions: pp. 91-115. Chapman \& Hall, London.

Williamson, M. (2003): Species-area relationships at small scales in continuum vegetation. - Journal of Ecology 91: 904-90.

Williamson, M., Gaston, K. J., Lonsdale, W. M. (2001): The species-area relationship does not have an asymptote! Journal of Biogeography 28: 827-830.

Williamson, M., Gaston, K. J., Lonsdale, W. M. (2002): An asymptote is an asymptote and not found in species-area relationships. - Journal of Biogeography 29: 1713-1713.

Willig, M. R., Kaufman, D. M., Stevens, R. D. (2003): Latitudinal gradients of biodiversity: pattern, process, scale, and synthesis. - Annual Review of Ecology, Evolution, and Systematics 34: 273-309.

Willner, W. (2006): The association concept revisited. - Phytocoenologia 36: 67-76.

Willner, W. (2011): Unambiguous assignment of relevés to vegetation units: the example of the Festuco-Brometea and Trifolio-Geranietea sanguinei. - Tuexenia 31:271-282 + 1 Tabelle.

Willner, W., Grabherr, G. (2007) [Eds.]: Die Wälder und Gebüsche Österreichs - Ein Bestimmungswerk mit Tabellen. - 2 Bände, $302+290$ pp. Spektrum Akademischer Verlag, Heidelberg.

Willner, W., Tichý, L., Chytrý, M. (2009): Effects of different fidelity measures and concepts on the determination of diagnostic species. - Journal of Vegetation Science 20: 130-137.

Wilson, E. O. (1988a) [Ed.]: Biodiversity. - 521 pp. National Academy Press, Washington, D. C.

Wilson, E. O. (1988b): The current state of biological diversity. - In: Wilson, E. O. [Ed.]: Biodiversity: pp. 3-18. National Academy Press, Washington, D. C.

Wilson, E. O. (1992) [Ed.]: Ende der biologischen Vielfalt? - Der Verlust an Arten, Genen und Lebensräumen und die Chancen für eine Umkehr. - 557 pp. Spektrum, Heidelberg.

Wisskirchen, R., Haeupler, H. (1998): Standardliste der Farn- und Blütenpflanzen Deutschlands. - 765 pp. Ulmer, Stuttgart.

World Resources Institute (2005) [Ed.]: Ecosystem and Human Well-Being: Biodiversity Synthesis - A Report of the Millennium Ecosystem Assessment. - 86 pp. World Resources Institute, Washington, DC.

Zehm, A., Nobis, M., Schwabe, A. (2003): Multiparameter analysis of vertical vegetation structure based on digital image processing. - Flora 198: 142-160. 


\section{Curriculum Vitae}

\section{Persönliche Daten}

Dr. Jürgen Dengler

Rückertstraße 3

D-21337 Lüneburg

Geboren am 12.06.1967 in Heilbronn am Neckar ledig

\section{Ausbildung}

19.02.2003 Promotion im Fach Ökologie an der Mathematisch-Naturwissenschaftlichen Fakultät der Christian-Albrechts-Universität zu Kiel über die Entwicklung und Bewertung neuer Ansätze in der Pflanzensoziologie unter besonderer Berücksichtigung der Vegetationsklassifikation (Referent: Prof. Dr. K. Dierßen, Kiel; Correferenten: Prof. Dr. W. Härdtle, Lüneburg, und Prof. Dr. E. Bergmeier, Göttingen)

17.06.1994 Diplom im Fach Biologie an der Christian-Albrechts-Universität zu Kiel mit einer Diplomarbeit zum Thema Trockenrasen im Biosphärenreservat Schorfheide-Chorin: Flora - Vegetation - Böden - Naturschutz bei Prof. Dr. K. Dierßen, Kiel

1991-1994 Studium von Botanik, Zoologie, Limnologie, Biologischer Meereskunde, Bodenkunde und Informatik an der Christian-Albrechts-Universität zu Kiel (6.-12. Fachsemester)

1988-1991 Studium der Biologie an der Universität Bayreuth (1.-5. Fachsemester)

19.06.1986 Abitur am Immanuel-Kant-Gymnasium in Leinfelden-Echterdingen (bei Stuttgart) mit der Gesamtnote 1,0

\section{Berufliche Tätigkeit}

10/2007-10/2011 Wissenschaftlicher Angestellter (Lehrvertretung Prof. Dr. N. Jürgens; Abteilung Biodiversität, Evolution und Ökologie der Pflanzen) am Biozentrum Klein Flottbek und Botanischer Garten, Universität Hamburg

2007

Werkauftrag im Rahmen der DFG-Biodiversitätsexploratorien an der Universität Potsdam

1998-2006 Wissenschaftlicher Angestellter am Institut für Ökologie und Umweltchemie, Fachbereich Umweltwissenschaften der Universität Lüneburg

1998-2004 Projektleitung beim Datenbank- und Buchprojekt „Die Pflanzengesellschaften Mecklenburg-Vorpommerns und ihre Gefährdung“" des Landesamtes für Umwelt, Naturschutz und Geologie Mecklenburg-Vorpommern (als Werkvertrag)

Seit 1993

Freiberufliche Tätigkeit als ökologischer Gutachter

1986-1988 Zivildienst beim Gartenbauamt der Landeshauptstadt Stuttgart (praktischer Naturschutz und Grünflächenpflege) und bei der Bezirksgruppe Stuttgart des Deutschen Bundes für Vogelschutz (Umweltbildung, Jugendarbeit, praktischer Naturschutz) 


\section{Auszeichnungen und Stipendien}

2003

Promotion zum Dr. rer. nat. mit summa cum laude an der MathematischNaturwissenschaftlichen Fakultät der Christian-Albrechts-Universität zu Kiel

1994-1997 Promotionsstipendium der Studienstiftung des deutschen Volkes

1991-1994 Stipendium der Studienstiftung des deutschen Volkes Diplom in Biologie mit Auszeichnung an der Christian-Albrechts-Universität zu Kiel

Mitgliedschaften in Editorial Boards von Fachzeitschriften

Web of Science Tuexenia

Sonstige $\quad$ Annali di Botanica, Diversity; Kieler Notizen zur Pflanzenkunde

\section{Gastherausgeberschaften in Fachzeitschriften}

In Vorbereitung Virtual Special Feature für Applied Vegetation Science zu Towards a consistent classification of European grasslands

In Vorbereitung Special Issue für Agriculture, Ecosystems and Environment zu Diversity patterns in European grasslands under the joint influence of nature and agriculture

2010-2012 Sonderband von Biodiversity \& Ecology zu Vegetation databases for the 21st century

2010-2011 Special Issue von Journal of Vegetation Science zu Ecoinformatics and global change

2010-2011 Special Feature von Plant Biosystems zu Succession, management and restoration of dry grasslands

2008 Sonderbandes der Mitteilungen der Arbeitsgemeinschaft Geobotanik in Schleswig-Holstein und Hamburg zu Flora, Vegetation and Nature Conservation from Schleswig-Holstein to South America - Festschrift for Klaus Dierßen on Occasion of his 60th Birthday

Seit 2005 Dry Grassland Special Features in Tuexenia (jährlich)

2004

Beiträge der 1. Trockenrasentagung in den Kieler Notizen zur Pflanzenkunde

Reviewer für Fachzeitschriften $(*=$ mehrfach)

Web of Science Agriculture, Ecosystems and Environment*; Applied Vegetation Science*; Basic and Applied Ecology*; Biodiversity and Conservation*; Biologia*; Biological Conservation; Community Ecology*; Ecography*; Ecological Indicators*; Flora; Folia Geobotanica*; Global Ecology and Biogeography*; Journal of Biogeography*; Journal of Applied Ecology; Journal of Ecology*; Journal of Vegetation Science*; Koedoe*; Landscape Ecology*; Methods in Ecology and Evolution; Naturwissenschaften; Oikos; Plant Ecology*; Plant Biosystems*; Plant Ecology \& Diversity; Preslia*; Theoretical Ecology; Tuexenia*

Sonstige $\quad$ Acta Botanica Croatica; Diversity; Frontiers of Biogeography; Kieler Notizen zur Pflanzenkunde*; Kochia; Mitteilungen der Arbeitsgemeinschaft Geobotanik in Schleswig-Holstein und Hamburg*; Web Ecology 


\section{Reviewer für wissenschaftliche Organisationen}

2011-2012 Ecological Society of America Panel on Vegetation Classification, Federal Geographic Data Committee of the USA \& NatureServe: National Vegetation Classification for the United States (USNVC) und International Vegetation Classification (IVC)

2011

Netherlands Organisation for Scientific Research: Projektantrag im Open Programme

\section{Leitungsfunktionen in wissenschaftlichen Organisationen}

Seit 2011

Gewähltes Mitglied im Council der International Association for Vegetation Science (IAVS)

Seit $2008 \quad$ Gründung und Leitung der European Dry Grassland Group (EDGG; www.edgg.org) gemeinsam mit S. Rusina, Lettland, M. Janišova, Slowakei, M. Vrahnakis, Griechenland, und S. Venn, Finnland. Die EDGG hat gegenwärtig rund 650 Mitgliedern aus 50 Ländern und ist seit Oktober 2009 eine offizielle Working Group der International Association for Vegetation Science (IAVS)

Seit $2007 \quad$ Vorstandsmitglied des Netzwerkes Phytodiversität Deutschland e. $V$. (NetPhyD); Gründung und Leitung der Sektion Taxonomische Referenzlisten

Seit 2005 Gründung und Leitung der internationalen Working Group on Dry Grasslands in the Nordic and Baltic Region

Seit 2004 Gründung und Leitung der Arbeitsgruppe Trockenrasen gemeinsam mit Dr. Ute Jandt, Halle (Saale)

\section{Organisation von wissenschaftlichen Tagungen und Symposien}

02.-03.11.2011 1st EuroSL Workshop: Towards establishing an electronic taxonomic reference list of all European plant taxa for use in vegetation databases and other plant-related databases in Göttingen

05.-08.09.2011 Symposium Biodiversity patterns at different scales - from theory to application auf der 41. Jahrestagung der Gesellschaft für Ökologie (GfÖ) in Oldenburg

24.-26.02.2010 9th international Meeting on Vegetation Databases in Hamburg unter dem Motto Vegetation Databases and Climate Change

6.-8.10.2009 Session Management and use of biodiversity auf dem Tropentag 2009, Hamburg

10.-14.09.2007 Symposien Scale-dependency in biodiversity und Grassland ecology, diversity, and conservation auf der 37. Jahrestagung der Gesellschaft für Ökologie (GfÖ) in Marburg

24.-26.09.2004 Erste Jahrestagung der Arbeitsgruppe Trockenrasen in Lüneburg unter dem Motto Trockenrasen als Biodiversitätshotspots

Sonstige wissenschaftliche Ämter

Seit 2010

Mitglied im Standing Committee der Phytosociological Nomenclature Commission der IAVS 
Seit 2010

Sprecher des Steering Committee des Global Index of Vegetation-Plot Databases (www.givd.info)

2008

Mitglied in der Auswahlkommission der Studienstiftung des deutschen Volkes

Seit 2007

2007

National Representative von SynBioSys Europe für Deutschland

Gutachter und Opponent im Promotionsverfahren von Dr. T. Černy an der

Karls-Universität Prag

\section{Eingeladene GastwissenschaftlerInnen aus dem Ausland}

2011

Salza Todorova, Masaryk University, Brno, Tschechien

2011

Prof. Dr. Emin Ugurlu, University of Manisa, Manisa, Türkei

2011

Prof. Dr. Alireza Naqinezhad, University of Mazandaran, Balbolsar, Iran

2011

Pavel Dan Turtureanu, Babes-Bolyai University, Cluj-Napoca, Rumänien

2010-2011

Bahar Behmanesh, Gorgan University of Agricultural Sciences and Natural Resources, Gorgan, Iran

2009

Hristo Pedashenko, Academy of Sciences, Sofia, Bulgarien

Mitgliedschaft in Fachorganisationen

International International Association for Vegetation Science (IAVS); Society for Conservation Biology (SCB); British Ecological Society (BES); Ecological Society of America (ESA), International Biogeography Society (IBS); Bryologischlichenologische Arbeitsgemeinschaft Mitteleuropas (BLAM); European Vegetation Survey (EVS); Workshop Vegetation Databases

National

Gesellschaft für Ökologie (GfÖ); Arbeitskreise „Makroökologie“ und „Renaturierungsökologie“ der GfÖ, Floristisch-soziologische Arbeitsgemeinschaft; Reinhold-Tüxen-Gesellschaft (RTG); Gesellschaft zur Erforschung der Flora Deutschlands (GEFD), Netzwerk Phytodiversität Deutschlands (NetPhyD)

Regional Botanische Vereine/Arbeitsgemeinschaften von Schleswig-Holstein und Hamburg, Hamburg, Mecklenburg-Vorpommern, Berlin und Brandenburg sowie Sachsen-Anhalt 


\section{Erläuterung des eigenen Anteils an den vorgelegten Publikatio- nen}

Bei den vorgelegten 38 Publikationen war ich 6-mal Alleinautor, 12-mal Erstautor, 8-mal Seniorautor und 12-mal sonstiger Mitautor.

\section{Quellen von Biodiversitätsdaten}

[1] Dengler, J. (2009b): A flexible, multi-scale approach for standardised recording of plant species richness patterns. - Ecological Indicators 9: 1169-1178.

\section{Alleinautor.}

[2] Jürgens, N., Schmiedel, U., Haarmeyer, D. H., Dengler, J., Finckh, M., Goetze, D., Gröngröft, A., Hahn, K., Koulibaly, A., Luther-Mosebach, J., Muche, G., Oldeland, J., Petersen, A., Porembski, S., Rutherford, M. C., Schmidt, M., Sinsin, B., Strohbach, B. J., Thiombiano, A., Wittig, R., Zizka, G. (im Druck): The BIOTA Biodiversity Observatories in Africa - A standardized framework for large-scale environmental monitoring. - Environmental Monitoring and Assessment. DOI: 10.1007/s10661-011-1993-y.

Mitautor: Während ich bei der Entwicklung des BIOTA-Erfassungsdesigns nicht beteiligt war, habe ich den vorliegenden Artikel maßgeblich mitkonzipiert und war einer der Hauptautoren. Mein Beiträge betrafen dabei vor allem die kritische Reflektion der verwendeten Methoden und ihren Vergleich mit anderen gängigen Biodiversitätsmonitoring-Programmen.

[3] Dengler, J., Ewald, J., Kühn, I., Peet, R. K. (2011a): Ecoinformatics and global change - an overdue liaison. - Journal of Vegetation Science 22: 577-581.

Erstautor: Dies ist das Editorial zu einem von mir initiierten und koordinierten Special Issue, das auf einer federführend von mir organisierten internationalen Tagung beruht. Die Konzeption des Textes stammt von mir, während J. Ewald und R. K. Peet einzelne Absätze beigesteuert haben.

[4] Dengler, J., Jansen, F., Glöckler, F., Peet, R. K., De Cáceres, M., Chytrý, M., Ewald, J., Oldeland, J., Finckh, M., Lopez-Gonzalez, G., Mucina, L., Rodwell, J. S., Schaminée, J. H. J., Spencer, N. (2011b): The Global Index of Vegetation-Plot Databases (GIVD): a new resource for vegetation science. - Journal of Vegetation Science 22: 582-597.

Erstautor: Dies isteine Analyse der vom Global Index of Vegetation-Plot Databases (GIVD) indizierten Datenbestände. Die Initiative zu GIVD stammt von mir und ich bin seit der Gründung Sprecher des GIVD Steering Committee. Den vorliegenden Artikel habe ich koordiniert, erhebliche Teile davon selbst verfasst und die Beiträge aller Co-AutorInnen eingearbeitet. 
[5] Jansen, F., Dengler, J., Glöckler, F., Chytrý, M., Ewald, J., Oldeland, J., Schaminée, J. H. J. (2011): Die mitteleuropäischen Datenbanken im Global Index of Vegetation-Plot Databases (GIVD). - Tuexenia 31: $351-367$.

Mitautor: Dieser Beitrag ist unter Federführung von F. Jansen in enger Anlehnung an Beitrag [4] entstanden. Der von F. Jansen entworfene Text wurde von mir vor der Einreichung kritisch überarbeitet.

[6] Jansen, F., Dengler, J. (2008): GermanSL - Eine universelle taxonomische Referenzliste für Vegetationsdatenbanken in Deutschland. - Tuexenia 28: 239-253.

Mitautor: GermanSL ist eine gemeinsame Idee von F. Jansen und mir, wobei ich stärker für das Konzept und die taxonomischen Inhalte zuständig bin, während F. Jansen federführend bei der informationstechnischen Implementierung ist. Wir haben diesen Artikel ungefähr zu gleichen Teilen verfasst und dann gemeinsam überarbeitet.

[7] Jansen, F., Dengler, J. (2010): Plant names in vegetation databases - a neglected source of bias. - Journal of Vegetation Science 21: 1179-1186.

Mitautor: Dieser Artikel verarbeitet im Kern Gedanken, die F. Jansen und ich gemeinsam bei der Erstellung der GermanSL entwickelt haben. Die Idee, diesen Artikel zu verfassen, stammt von F. Jansen, doch haben wir sie dann ungefähr zu gleichen Teilen in die Praxis umgesetzt .

\section{Theorie, Simulationen und Analysemethoden zur Skalenabhängigkeit von Biodiversität}

[8] Dengler, J. (2009a): Which function describes the species-area relationship best? - A review and empirical evaluation. - Journal of Biogeography 36: 728-744.

Alleinautor.

[9] Dengler, J. (2008): Pitfalls in small-scale species-area sampling and analysis. - Folia Geobotanica 43: 269287.

Alleinautor.

[10] Dengler, J., Oldeland, J. (2010): Effects of sampling protocol on the shapes of species richness curves. Journal of Biogeography 37: 1698-1705.

Erstautor: Die Idee zu diesem Artikel stammt von mir, ich habe die statistischen Analysen der Ergebnisse der von J. Oldeland nach meinen Vorgaben erstellten Simulationen durchgeführt und den Text verfasst, der dann von J. Oldeland Korrektur gelesen wurde.

[11] Dengler, J., Boch, S. (2008b): Sampling-design effects on properties of species-area curves - a case study from Estonian dry grassland communities. - Folia Geobotanica 43: 289-304.

Erstautor: Die Datengrundlage für diesen Artikel hat S. Boch in einer von mir konzipierten und betreuten Diplomarbeit geschaffen (Boch 2005). Die Idee zu diesem Artikel stammt von mir, ich habe die stastischen Analysen durchgeführt und den Text verfasst, der dann von S. Boch kritisch durchgesehen wurde. 
[12] Dengler, J. (2010): Robust methods for detecting a small island effect. - Diversity and Distributions 16: 256266.

Alleinautor.

[13] Dengler, J., Löbel, S., Dolnik, C. (2009): Species constancy depends on plot size - a problem for vegetation classification and how it can be solved. - Journal of Vegetation Science 20: 754-766.

Erstautor: Datengrundlage für diesen Artikel waren neben publierten Veröffentlichungen unpublizierte Daten von C. Dolnik und aus der von mir konzipierten und betreuten Diplomarbeit von S. Löbel (Löbel 2002). Die Idee zu vorliegendem Artikel und das zu Grunde liegende mathematische Konzept stammen von mir. Ich habe die statistischen Analysen durchgeführt und den Text verfasst, der anschließend von beiden MitautorInnen kritisch durchgesehen wurde.

\section{Artendiversität auf verschiedenen Skalenebenen}

[14] Löbel, S., Dengler, J., Hobohm, C. (2004): Beziehungen zwischen der Artenvielfalt von Gefäßpflanzen, Moosen und Flechten in Trockenrasen der Insel Öland (Schweden). - Kieler Notizen zur Pflanzenkunde Schleswig-Holstein und Hamburg 32: 9-13.

Mitautor: Die Datengrundlage für diesen Artikel hat S. Löbel in einer von mir konzipierten und betreuten Diplomarbeit geschaffen (Löbel 2002). Den vorliegenden Artikel hat S. Löbel federführend verfasst und ich habe den Text vor der Einreichung kritisch durchgesehen.

[15] Dengler, J. (2005): Zwischen Estland und Portugal - Gemeinsamkeiten und Unterschiede der Phytodiversitätsmuster europäischer Trockenrasen. - Tuexenia 25: 387-405.

Alleinautor.

[16] Allers, M.-A., Dengler, J. (2007): Small-scale patterns of plant species richness in the central European landscape. - Verhandlungen der Gesellschaft für Ökologie 37: 181.

Seniorautor: Die Datengrundlage für diesen Artikel hat M.-A. Allers in einer von mir konzipierten und betreuten Diplomarbeit geschaffen (Allers 2007). Das vorliegende „extended summary“ habe ich federführend verfasst, mit kritischer Durchsicht von M.-A. Allers.

[17] Dengler, J. (2006): Variabilität von Artendichte und Artenzusammensetzung auf unterschiedlichen räumlichen Skalenebenen - Exemplarische Untersuchungen aus Trockenrasen und Konsequenzen für das Probedesign von Biodiversitätsuntersuchungen. - In: Bültmann, H., Fartmann, T., Hasse, T. [Eds.]: Trockenrasen auf unterschiedlichen Betrachtungsebenen. - Arbeiten aus dem Institut für Landschaftsökologie Münster 15: 7381. Münster.

\section{Alleinautor.}


[18] Turtureanu, P. D., Dengler, J. (im Druck): Different aspects of plant diversity show contrasting patterns in Carpathian forest openings. - Plant Ecology. DOI: 10.1007/s11258-011-0007-z.

Seniorautor: Diese Arbeit ist gedacht als Bestandteil der kumulativen Dissertation von P. D. Turtureanu. Während ich bei der Konzeption und Durchführung der Geländeerhebungen für diesen Artikel nicht beteiligt war, habe ich P. D. Turtureanu während seines Gastaufenthaltes in Hamburg (auf meine Einladung hin) umfangreich bei der Konzeption und Durchführung der Analysen und beim Verfassen des Artikels beraten und diesen etliche Male kritisch durchgesehen und überarbeitet.

[19] Löbel, S., Dengler, J., Hobohm, C. (2006): Species richness of vascular plants, bryophytes and lichens in dry grasslands: The effects of environment, landscape structure and competition. - Folia Geobotanica 41: 377393.

Mitautor: Die Datengrundlage für diesen Artikel hat S. Löbel in einer von mir konzipierten und betreuten Diplomarbeit geschaffen (Löbel 2002). Sie hat die Analysen mit meiner Beratung konzipiert und durchgeführt und den Artikel federführend verfasst, während ich ihn vor der Ersteinreichung kritisch durchgesehen und nach erfolgter Begutachtung die notwendige Revision vorgenommen habe.

[20] Ruprecht, E., Szabó, A., Enyedi, M. Z., Dengler, J. (2009): Steppe-like grasslands in Transylvania (Romania): characterisation and influence of management on species diversity and composition. - Tuexenia 29: 353-368 + 1 Tabelle.

Seniorautor: Die Konzeption und Durchführung der Geländeerhebungen zu diesem Artikel erfolgte ohne meine Beteiligung. Ich war dann aber wesentlich bei der Konzeption der Analysen, insbesondere bei der Berücksichtigung der Skalenabhängigkeit von Biodiversität und bei der Ermittlung diagnostischer Arten beteiligt. Der Text wurde federführend von E. Ruprecht verfasst, doch vor den Einreichungen jeweils umfangreich durch mich überarbeitet.

[21] Vassilev, K., Pedashenko, H., Nikolov, S. C., Apostolova, I., Dengler, J. (2011): Effect of land abandonment on the vegetation of upland semi-natural grasslands in the Western Balkan Mts., Bulgaria. - Plant Biosystems 145: 654-665.

Seniorautor: Die Konzeption und Durchführung der Geländeerhebungen zu diesem Artikel erfolgte ohne meine Beteiligung. Ich habe dann aber einen wesentlichen Teil der statistischen Analysen konzipiert und durchgeführt und mich in erheblichem Umfang an der konzeptionellen Ausrichtung und der mehrfachen Überarbeitung des Textes beteiligt. Dies geschah teilweise während des Gastaufenthaltes von H. Pedashenko in Hamburg (auf meine Einladung hin).

[22] Friedel, A., Oheimb, G. von, Dengler, J., Härdtle, W. (2006): Species diversity and species composition of epiphytic bryophytes and lichens - a comparison or managed and unmanaged beech forests in NE Germany. - Feddes Repertorium 117: 172-185.

Mitautor: Diese Arbeit ist Bestandteil der kumulativen Dissertation von A. Friedel. Die Konzeption und Durchführung der Geländeerhebungen zu diesem Artikel erfolgte ohne meine Beteiligung. Ich war dann aber 
an der Bestimmung einiger Moose und wesentlich an der Konzeption und Durchführung eines Teils der Analysen (insbesondere der korrekten Berücksichtigung der Skalenabhängigkeit von Biodiversität) beteiligt und habe zur kritischen Durchicht und Überarbeitung des Textes beigetragen.

[23] Schmiedel, U., Dengler, J., Luther-Mosebach, J., Gröngröft, A., Muche, G., Petersen, A., Strohbach, B. J., Jürgens, N. (2010): Patterns and dynamics of vascular plant diversity along the BIOTA transects in southern Africa. - In: Schmiedel, U., Jürgens, N. [Eds.]: Biodiversity in Southern Africa. Volume 2: Patterns and Processes at Regional Scale: pp. 118-135. Klaus Hess Publishers, Göttingen \& Windhoek.

Mitautor: Die Konzeption und Durchführung der Geländeerhebungen zu diesem Artikel erfolgte ohne meine Beteiligung. Ich habe dann aber sämtliche statistischen Auswertungen konzipiert und durchgeführt und den größten Teil des Textes verfasst. Die zweite Hauptautorin war U. Schmiedel, während die Beiträge aller übrigen Co-AutorInnen sich im Wesentlichen auf die kritische Durchsicht der von uns beiden entworfenen Texte beschränkte. Dass in diesem Beitrag die Skalenabhängigkeit von Biodiversität umfangreich und methodisch einwandfrei analysiert wurde, geht auf mich zurück, genauso wie der Vergleich der im südlichen Afrika gefundenen Biodiversitätsmuster mit jenen aus anderen Erdregionen.

[24] Haarmeyer, D. H., Schmiedel, U., Dengler, J., Bösing, B. M. (2010a): How does grazing intensity affect different vegetation types in arid Succulent Karoo, South Africa? Implications for conservation management. - Biological Conservation 143: 588-596.

Mitautor: Die Grundlage dieser Veröffentlichung ist die Diplomarbeit von D. H. Haaremeyer (Haarmeyer 2009), die von U. Schmiedel konzipiert und von ihr und mir gemeinsam betreut wurde. Meine Beiträge waren dabei vor allem methodischer Art, insbesondere bezüglich statistischer Verfahren, Biodiversitätsanalysen und Skalenabhängigkeiten. Der vorliegende Artikel wurde federführend von D. H. Haarmeyer konzipiert und verfasst, mit intensivem Feedback von U. Schmiedel und mir.

[25] Schmiedel, U., Dengler, J., Etzold, S. (im Druck): Vegetation dynamics of endemic-rich quartz fields in the Succulent Karoo, South Africa, in response to recent climatic trends. - Journal of Vegetation Science. DOI: 10.1111/j.1654-1103.2011.01346.x.

Mitautor: Die Konzeption und Durchführung der Geländeerhebungen zu diesem Artikel erfolgte ohne meine Beteiligung durch die beiden anderen Autorinnen. Ich habe die statistischen Analysen konzipiert und durchgeführt und den Methods- und Results-Teil der Arbeit größtenteils verfasst. Gemeinsam mit den beiden Mitautorinnen habe ich den Gesamtext bei der Ersteinreichung und bei den Revisionen kritisch durchgelesen und überarbeitet.

\section{Analyse und Diversität von Pflanzengesellschaften}

[26] Dengler, J., Chytrý, M., Ewald, J. (2008): Phytosociology. - In: Jørgensen, S. E., Fath, B. D. [Eds.]: Encyclopedia of Ecology: pp. 2767-2779. Elsevier, Oxford.

Erstautor: Ich habe die Einladung zu diesem Artikel von der Encyclopedia of Ecology erhalten und daraufhin das dreiköpfige Autorenteam zusammengestellt. Die Konzeption des Artikels und die 
Arbeitsteilung zwischen den Autoren habe ich koordiniert. Jeder der drei Autoren hat ungefähr 1/3 des Textes federführend verfasst.

[27] Dengler, J., Berg, C., Jansen, F. (2005): New ideas for modern phytosociological monographs. - Annali di Botanica N. S. 5: 49-66.

Erstautor: Dieser Beitrag fußt zu erheblichen Teilen auf von mir entwickelten konzeptionellen Ideen, die dann in den „Pflanzengesellschaften Mecklenburg-Vorpommerns“ (Berg et al. 2004), wo ich maßgeblich für die Klassifikationsmethodik zuständig war, erstmals umfangreich erprobt wurden. Ich hatte die Federführung beim Verfassen und Überarbeiten des vorliegenden Artikels, doch gab es auch umfangreiche Beiträge von C. Berg und kleinere von F. Jansen.

[28] Berg, C., Dengler, J. (2005): Moose und Flechten als diagnostische Arten von Pflanzengesellschaften - eine Übersicht aus Mecklenburg-Vorpommern. - Herzogia 18: 145-161.

Mitautor: Dieser Beitrag fußt auf den „Pflanzengesellschaften Mecklenburg-Vorpommerns“ (Berg et al. 2004), wo ich maßgeblich für die Klassifikationsmethodik zuständig war. Die Idee zu vorliegendem Artikel hatte C. Berg, während wir den Text ungefähr zu gleichen Teilen verfasst haben.

[29] Timmermann, T., Dengler, J., Abdank, A., Berg, C. (2006): Objektivierung von Naturschutzbewertungen Das Beispiel Roter Listen von Pflanzengesellschaften. - Naturschutz und Landschaftsplanung 38: $133-139$.

Mitautor: Dieser Beitrag fußt auf den „Pflanzengesellschaften Mecklenburg-Vorpommerns“ (Berg et al. 2004), wo alle vier AutorInnen die Methodik der Naturschutzbewertungen entwickelt haben. Beim Verfassen des vorliegenden Beitrags hatte T. Timmermann die Federführung, doch haben die drei MitautorInnen jeweils umfangreiche und ähnlich große Beiträge zum Text und dessen Überarbeitung geleistet.

[30] Dengler, J., Löbel, S. (2006): The basiphilous dry grasslands of shallow, skeletal soils (Alysso-Sedetalia) on the island of Öland (Sweden), in the context of North and Central Europe. - Phytocoenologia 36: 343-391.

Erstautor: Die wichtigste Grundlage dieser Veröffentlichung ist die Diplomarbeit von S. Löbel (Löbel 2002), die von mir konzipiert und betreut wurde. Ergänzend haben S. Löbel und ich noch umfangreiche Literaturdaten digitalisiert. Den Artikel haben S. Löbel und ich gemeinsam konzipiert und jeder von uns hat einen Teil der Analysen und Datenaufbereitungen durchgeführt. Beim Verfassen des Textes hatte ich die Federführung, doch hat S. Löbel dazu Beiträge in nahezu gleichem Umfang geleistet.

[31] Dengler, J., Löbel, S., Boch, S. (2006b): Dry grassland communities of shallow, skeletal soils (SedoScleranthenea) in northern Europe. - Tuexenia 26: 159-190 + 6 Tabellen.

Erstautor: Die wichtigste Grundlage dieser Veröffentlichung sind die Diplomarbeiten von S. Löbel (Löbel 2002) und S. Boch (Boch 2005), die beide von mir konzipiert und betreut wurden. Beim vorliegenden Artikel und den zugrundeliegenden Datenaufbereitungen und Analysen hatte ich die Federführung, doch haben beide Co-AutorInnen wesentliche Beiträge geleistet. 
[32] Boch, S., Dengler, J. (2006): Floristische und ökologische Charakterisierung sowie Phytodiversität der Trockenrasen auf der Insel Saaremaa (Estland). - In: Bültmann, H., Fartmann, T., Hasse, T. [Eds.]: Trockenrasen auf unterschiedlichen Betrachtungsebenen. - Arbeiten aus dem Institut für Landschaftsökologie Münster 15: 55-71. Münster.

Seniorautor: Dieser Artikel basiert auf der von mir konzipierten und betreuten Diplomarbeit von S. Boch (Boch 2005). S. Boch hat diesen Artikel dann federführend verfasst, mit konstruktiv-kritischer Begleitung meinerseits.

[33] Löbel, S., Dengler, J. (2008) [,2007’]: Dry grassland communities on southern Öland: phytosociology, ecology, and diversity. - In: van der Maarel, E. [Ed.]: Structure and dynamics of alvar vegetation on Öland and some related dry grasslands - Dedicated to Ejvind Rosén on his $65^{\text {th }}$ birthday. - Acta Phytogeographica Suecica 88: 13-32. Svenska Växtgeografiska Sällskapet, Uppsala.

Seniorautor: Dieser Artikel basiert auf der von mir konzipierten und betreuten Diplomarbeit von S. Löbel (Löbel 2002). S. Löbel hat diesen Artikel dann federführend verfasst, mit konstruktiv-kritischer Begleitung meinerseits.

[34] Dengler, J., Eisenberg, M., Schröder, J. (2006a): Die grundwasserfernen Saumgesellschaften Nordostniedersachsens im europäischen Kontext - Teil I: Säume magerer Standorte (Trifolio-Geranietea sanguinei). - Tuexenia 26: 51-93 + 9 Tabellen.

Erstautor: Die Arbeit basiert auf Daten aus den von mir konzipierten und betreuten Diplomarbeiten von M. Eisenberg und J. Schröder (geb. Krebs) in Kombination mit umfangreichen unpublizierten Daten von mir. Die Idee zu zu diesem Artikel stammt von mir, ich hatte die Federführung beim endgültigen Aufbereiten der Daten und Verfassen des Manuskriptes und war für die Revision nach erfolgter Begutachtung verantwortlich.

[35] Dengler, J., Eisenberg, M., Schröder, J. (2007): Die grundwasserfernen Saumgesellschaften Nordostniedersachsens im europäischen Kontext - Teil II: Säume nährstoffreicher Standorte (Artemisietea vulgaris) und vergleichende Betrachtung der Saumgesellschaften insgesamt. - Tuexenia 27: 91-136 + 6 Tabellen.

Erstautor: Die Arbeit basiert auf Daten aus den von mir konzipierten und betreuten Diplomarbeiten von $M$. Eisenberg und J. Schröder (geb. Krebs) in Kombination mit umfangreichen unpublizierten Daten von mir. Die Idee zu zu diesem Artikel stammt von mir, ich hatte die Federführung beim endgültigen Aufbereiten der Daten und Verfassen des Manuskriptes und war für die Revision nach erfolgter Begutachtung verantwortlich.

[36] Dengler, J., Boch, S. (2008a): Forest-edge communities (Trifolio-Geranietea sanguinei) on the island of Saaremaa (Estonia): Phytosociology and biodiversity patterns. - In: Dengler, J., Dolnik, C., Trepel, M. [Eds.]: Flora, Vegetation und Naturschutz zwischen Schleswig-Holstein und Südamerika - Festschrift für Klaus Dierßen zum 60. Geburtstag. - Mitteilungen der Arbeitsgemeinschaft Geobotanik in SchleswigHolstein und Hamburg 65: 257-286 + 2 Beilagen. Kiel. 
Erstautor: Die Idee zu diesem Artikel stammt von mir, die zugrundeliegenden Vegetationsaufnahmen wurden teils von mir, teils von $S$. Boch, teils von uns gemeinsam erhoben. Ich habe die Analysen durchgeführt und den Text größtenteils verfasst, während S. Boch kleinere Beiträge beigesteuert hat.

[37] Michl, T., Dengler, J., Huck, S. (2010): Montane-subalpine tall-herb vegetation (Mulgedio-Aconitetea) in central Europe: large-scale synthesis and comparison with northern Europe. - Phytocoenologia 40: 117-154.

Seniorautor (wir haben uns hier entschlossen, mich als Seniorautor an zweiter Stelle zu listen, da ich zugleich erheblich mehr Beiträge als der Drittautor geleistet habe): Dieser Artikel ist Bestandteil der kumulativen Dissertation von T. Michl. Ich war erheblich in Konzeption der Analysen und das Verfassen und Überbeiten des Textes involviert, wobei ich insbesondere meine methodischen Kompetenzen bei der überregionalen konsistenten Vegetationsklassifikation und bei der pflanzensoziologischen Nomenklatur eingebracht habe.

[38] Luther-Mosebach, J., Dengler, J., Schmiedel, U., Röwer, I. U., Labitzky, T., Gröngröft, A. (im Druck): A first formal classification of Hardeveld vegetation in Namaqualand, South Africa. - Applied Vegetation Science. DOI: 10.1111/j.1654-109X.2011.01173.x.

Seniorautor (wir haben uns hier entschlossen, mich als Seniorautor an zweiter Stelle zu listen, da ich zugleich erheblich mehr Beiträge als die folgenden Autoren geleistet habe): Dieser Artikel basiert auf den von J. Luther-Mosebach und I. U. Röwer im Rahmen von deren Diplomarbeiten (Luther-Mosebach 2009, Röwer 2009) erhobenden Daten. Die Diplomarbeiten wurden von U. Schmiedel und mir gemeinsam konzipiert und betreut. Die methodische Herangehensweise an die Vegetationsklassifikation in einem zuvor syntaxonomisch nicht bearbeiteten Gebiet wurde von mir entwickelt und dann von J. Luther-Mosebach umgesetzt, ebenso alle statistischen Analysen. Der Text wurde ungefähr zu gleichen Teilen von J. LutherMosebach, U. Schmiedel und mir verfasst, während ich nach erfolgter Begutachtung die notwendige Revision weitgehend alleine vorgenommen habe. 


\section{Gesamtpublikationsverzeichnis}

Bis zum Zeitpunkt der Fertigstellung dieser Habilitationsschrift habe ich (ohne Buchbesprechungen) insgesamt 158 wissenschaftliche Publikationen (ohne Buchbesprechungen) verfasst, wovon 149 erschienen, vier im Druck und fünf im Review-Verfahren sind. Bei 29 Publikationen (21 erschienen, 4 im Druck, 4 eingereicht) handelt es sich um Artikel für/in Zeitschriften, die im Web of Science (http://apps.webofknowledge.com/) geführt sind. Weiterhin habe ich sechs Bücher (mit-) herausgegeben und/oder verfasst. Die in die Habilitationsschrift einbezogenen Publikationen sind fett gesetzt.

\section{III.1 Publikationen im Web of Science}

\section{Zur Veröffentlichung eingereicht}

Akasbi, Z., Oldeland, J., Dengler, J., Finckh, M.: Advances in the analysis of GPS tracks for the assessment of grazing pattern and intensity: a case study with goats in southern Morocco. - Manuskript für Environmental Monitoring and Assessment (im Review).

Akasbi, Z., Oldeland, J., Dengler, J., Finckh, M.: Effects of grazing exclusion on interannual changes of dwarf shrub biomass in a Moroccan sagebrush steppe. - Manuskript für African Journal of Range and Forage Science (im Review).

Beck, J., Ballesteros-Mejia, L., Buchmann, C. M., Dengler, J., Fritz, S., Gruber, B., Hof, C., Jansen, F., Knapp, S., Kreft, H., Schneider, A.-K., Winter, M., Dormann, C. F.: What's on the horizon for macroecology? Manuskript for Ecography (in Revision nach positiver Begutachtung).

Wilson, J. B., Peet, R. K., Dengler, J., Pärtel, M.: Plant species richness: the world records. - Manuskript für Journal of Vegetation Science (wieder eingereicht nach positiver Begutachtung).

\section{Im Druck}

Jürgens, N., Schmiedel, U., Haarmeyer, D. H., Dengler, J., Finckh, M., Goetze, D., Gröngröft, A., HahnHadjali, K., Koulibaly, A., Luther-Mosebach, J., Muche, G., Oldeland, J., Petersen, A., Porembski, S., Rutherford, M. C., Schmidt, M., Sinsin, B., Strohbach, B. J., Thiombiano, A., Wittig, R., Zizka, G. (im Druck): The BIOTA Biodiversity Observatories in Africa - A standardized framework for large-scale environmental monitoring. - Environmental Monitoring and Assessment. DOI: 10.1007/s10661-011-1993-y.

Luther-Mosebach, J., Dengler, J., Schmiedel, U., Röwer, I. U., Labitzky, T., Gröngröft, A. (im Druck): A first formal classification of Hardeveld vegetation in Namaqualand, South Africa. - Applied Vegetation Science. DOI: 10.1111/j.1654-109X.2011.01173.x.

Schmiedel, U., Dengler, J., Etzold, S. (im Druck): Vegetation dynamics of endemic-rich quartz fields in the Succulent Karoo, South Africa, in response to response to recent climatic trends. - Journal of Vegetation Science. DOI: 10.1111/j.1654-1103.2011.01346.x.

Turtureanu, P. D., Dengler, J. (im Druck): Different aspects of plant diversity show contrasting patterns in Carpathian forest openings. - Plant Ecology. DOI: 10.1007/s11258-011-0007-z. 


\section{1}

Dengler, J., Ewald, J., Kühn, I., Peet, R. K. (2011): Ecoinformatics and global change - an overdue liason. Journal of Vegetation Science 22: 577-581.

Dengler, J., Jansen, F., Glöckler, F., Peet, R. K., De Cáceres, M., Chytrý, M., Ewald, J., Oldeland, J., Finckh, M., Lopez-Gonzalez, G., Mucina, L., Rodwell, J. S., Schaminée, J. H. J., Spencer, N. (2011): The Global Index of Vegetation-Plot Databases (GIVD): a new resource for vegetation science. - Journal of Vegetation Science 22: 582-597.

Janišová, M., Bartha, S., Kiehl, K., Dengler, J. (2011): Advances in the conservation of dry grasslands Introduction to contributions from the 7th European Dry Grassland Meeting. - Plant Biosystems 145: 507-513.

Janišová, M., Wellstein, C., Willner, W., Dengler, J. (2011): Succession, restoration, and management of dry grasslands - Special Feature with contributions from the 7th European Dry Grassland Meeting 2010 in Smolenice. - Tuexenia 31: 227-234.

Jansen, F., Dengler, J., Glöckler, F., Chytrý, M., Ewald, J., Oldeland, J., Schaminée, J. H. J. (2011): Die mitteleuropäischen Datenbanken im Global Index of Vegetation-Plot Databases (GIVD). - Tuexenia 31: 351-367.

Vassilev, K., Pedashenko, H., Apostolova, I., Nikolov, S. C., Dengler, J. (2011): Effect of land abandonment on the vegetation of upland semi-natural grasslands in the Western Balkan Mts., Bulgaria. - Plant Biosystems 145: 654-665.

\section{0}

Dengler, J. (2010): Robust methods for detecting a small island effect. - Diversity and Distributions 16: 256266.

Dengler, J., Oldeland, J. (2010): Effects of sampling protocol on the shapes of species richness curves. Journal of Biogeography 37: 1698-1705.

Haarmeyer, D. H., Bösing, B. M., Schmiedel, U., Dengler, J. (2010): The role of domestic herbivores in endozoochorous plant dispersal in the arid Knersvlakte, South Africa. - South African Journal of Botany 76: $359-364$.

Haarmeyer, D. H., Schmiedel, U., Dengler, J., Bösing, B. M. (2010): How does grazing intensity affect different vegetation types in arid Succulent Karoo, South Africa? Implications for conservation management. - Biological Conservation 143: 588-596.

Jansen, F., Dengler, J. (2010): Plant names in vegetation databases - a neglected source of bias. - Journal of Vegetation Science 21: 1179-1186.

Michl, T., Dengler, J., Huck, S. (2010): Montane-subalpine tall-herb vegetation (Mulgedio-Aconitetea) in central Europe: large-scale synthesis and comparison with northern Europe. - Phytocoenologia 40: 117154.

2009

Dengler, J., Löbel, S., Dolnik, C. (2009): Species constancy depends on plot size - a problem for vegetation classification and how it can be solved. - Journal of Vegetation Science 20: 754-766.

Dengler, J. (2009): A flexible, multi-scale approach for standardised recording of plant species richness patterns. - Ecological Indicators 9: 1169-1178. 
Dengler, J. (2009): Which function describes the species-area relationship best? - A review and empirical evaluation. - Journal of Biogeography 36: 728-744.

\section{8}

Dengler, J. (2008): Pitfalls in small-scale species-area sampling and analysis. - Folia Geobotanica 43: $269-287$.

Dengler, J., Boch, S. (2008): Sampling-design effects on properties of species-area curves - a case study from Estonian dry grassland communities. - Folia Geobotanica 43: 289-304.

Putfarken, D., Dengler, J., Lehmann, S., Härdtle, W. (2008): Site use of grazing cattle and sheep in a large-scale pasture landscape: a GPS/GIS assessment. - Applied Animal Behaviour Science 111: 54-67.

\section{7}

Dengler, J., Boch, S. (2007): Taxonomy, ecology, and distribution of six remarkable plant taxa (Spermatophyta, Bryophyta, and Lichenes) of the Estonian dry grassland flora. - Phyton 47: 47-71.

\section{6}

Dengler, J., Löbel, S. (2006): The basiphilous dry grasslands of shallow, skeletal soils (Alysso-Sedetalia) on the island of Öland (Sweden), in the context of North and Central Europe. - Phytocoenologia 36: 343-391.

Löbel, S., Dengler, J., Hobohm, C. (2006): Species richness of vascular plants, bryophytes and lichens in dry grasslands: The effects of environment, landscape structure and competition. - Folia Geobotanica 41: 377-393.

\section{III.2 Buchpublikationen}

\section{0}

Jürgens, N., Haarmeyer, D. H., Luther-Mosebach, J., Dengler, J., Finckh, M., Schmiedel, U. (2010) [Eds.]: Biodiversity in Southern Africa. Volume 1: Patterns at Local Scale - the BIOTA Observatories. - XX $+801 \mathrm{pp}$. Klaus Hess Publishers, Göttingen \& Windhoek.

darin:

Haarmeyer, D. H., Luther-Mosebach, J., Dengler, J., Schmiedel, U., Finckh, M., Berger, K., Deckert, J., Domptail, S. E., Dreber, N., Gibreel, T., Grohmann, C., Gröngröft, A., Haensler, A., Hanke, W., Hoffmann, A., Husted, L. B., Kangombe, F. N., Keil, M., Krug, C. B., Labitzky, T., Linke, T., Mager, D., Mey, W., Muche, G., Naumann, C., Pellowski, M., Powrie, L. W., Pröpper, M., Rutherford, M. C., Schneiderat, U., Strohbach, B. J., Vohland, K., Weber, B., Wesuls, D., Wisch, U., Zedda, L., Büdel, B., Darienko, T., Deutschewitz, K., Dojani, S., Erb, E., Falk, T., Friedl, T., Kanzler, S.-E., Limpricht, C., Linsenmair, K. E., Mohr, K., Oliver, T., Petersen, A., Rambold, G., Zeller, U., Austermühle, R., Bausch, J., Bösing, B. M., Classen, N., Dorendorf, J., Dorigo, W., Esler, K. J., Etzold, S., Graiff, A., Grotehusmann, L., Hecht, J., Hoyer, P., Kongor, R. Y., Lang, H., Lieckfeld, L. A. B., Oldeland, J., Peters, J., Röwer, I. U., September, Z. M., Sop, T. K., van Rooyen, M. W., Weber, J., Willer, J., Jürgens, N. (2010): The BIOTA Observatories. - pp. 6-801. 


\section{8}

Dengler, J., Dolnik, C., Trepel, M. (2008) [Eds.]: Flora, Vegetation und Naturschutz zwischen Schleswig-Holstein und Südamerika - Festschrift für Klaus Dierßen zum 60. Geburtstag. - Mitteilungen der Arbeitsgemeinschaft Geobotanik in Schleswig-Holstein und Hamburg 65: 526 pp. + 4 Beilagen. Kiel.

\section{6}

Schulz, F., Dengler, J. (2006) [Eds.]: Verbreitungsatlas der Moose in Schleswig-Holstein und Hamburg. - 402 pp. Landesamt für Natur und Umwelt des Landes Schleswig-Holtein, Flintbek.

darin:

Dengler, J., Schulz, F.: Über das Projekt. - pp. 10-12.

Dierßen, K., Martin, C., Lütt, S., Dolnik, C., Siemsen, M., Dengler, J.: Mooslebensräume im Gebiet. - pp. 1844.

Lütt, S., Dengler, J., Martin, C., Schulz, F.: Gefährdung und Schutz der Moose im Gebiet. - pp. 45-54.

Dierßen, K., Dengler, J.: Moose in Pflanzengesellschaften und Synusien. - pp. 55-56.

Dengler, J.: Systematik und Nomenklatur der Sippen. - pp. 65-68.

Schulz, F., Dengler, J.: Bilanz der Kartierung. - pp. 68-78.

Dengler, J., Schulz, F.: Aufbau des Speziellen Teils. - pp. 79-82.

Siemsen, M., Dierßen, K., Schulz, F., Dolnik, C., Dengler, J., Lütt, S., Martin, C., Meinunger, L., Schröder, W.: Spezieller Teil. - pp. 83-360.

Dengler, J., Dierßen, K.: Syntaxonomische Übersicht der Pflanzengesellschaften im Gebiet. - pp. 368-371.

Dengler, J., Dierßen, K., Martin, C., Siemsen, M.: Glossar. - pp. 373-382.

\section{4}

Berg, C., Dengler, J., Abdank, A., Isermann, M. (2004) [Eds]: Die Pflanzengesellschaften MecklenburgVorpommerns und ihre Gefährdung - Textband. - 606 pp. Weissdorn, Jena.

darin:

Berg, C., Dengler, J., Abdank, A., Isermann, M.: Über dieses Projekt. - pp. 10-14.

Berg, C., Dengler, J., Abdank, A., Isermann, M.: Hinweise zum Aufbau des Werkes. - pp. 14-16.

Isermann, M., Dengler, J.: Introduction and summary for English-speaking readers. - pp. 16-21.

Dengler, J., Berg, C.: Vegetationsklassifikation. - pp. 29-40.

Dengler, J.: Nomenklatur der Pflanzengesellschaften. - pp. 41-51.

Dengler, J., Berg, C.: Benennung der Pflanzen. - pp. 53-54.

Berg, C., Dengler, J.: Verbreitungskarten von Syntaxa. - pp. 57-60.

Berg, C., Timmermann, T., Dengler, J.: Naturschutzfachliche Wertstufe. - pp. 67-72.

Abdank, A., Dengler, J., Berg, C.: Handlungsbedarf. - pp. 72-73.

Dengler, J., Wollert, H.: Klasse: Polygono-Poetea annuae Rivas-Martínez 1975 - Annuellen-Trittrasen und verwandte Gesellschaften. - pp. 257-263.

Dengler, J., Wollert, H.: Klasse: Sisymbrietea Korneck 1974 nom. cons. propos. - Annuellen-Ruderalfluren frischer bis trockener Standorte. - pp. 264-272.

Dengler, J.: Klasse: Koelerio-Corynephoretea Klika in Klika \& V. Novák 1941 - Sandtrockenrasen und Felsgrusfluren von der submeridionalen bis zur borealen Zone. - pp. 301-326. 
Dengler, J.: Klasse: Festuco-Brometea Br.-Bl. \& Tx. ex Klika \& Hadač 1944 - Basiphile Magerrasen und Steppen im Bereich der submeridionalen und temperaten Zone. - pp. 327-335.

Dengler, J.: Klasse: Trifolio-Geranietea sanguinei T. Müller 1962 - Licht- und wärmebedürftige Saumgesellschaften und Staudenfluren magerer Standorte. - pp. 362-379.

Dengler, J., Wollert, H.: Klasse: Artemisietea vulgaris Lohmeyer \& al. ex von Rochow 1951 - Ausdauernde Ruderalgesellschaften und Säume frischer bis trockener, stickstoffreicher Standorte. - pp. 380-410.

Abdank, A., Berg, C., Dengler, J., Isermann, M.: Bilanz der Roten Liste und Konsequenzen für den Naturschutz. - pp. 494-507.

Isermann, M., Berg, C., Dengler, J., Schmidt, J.: Glossar. - pp. 518-523.

2003

Dengler, J. (2003, korrigierter Nachdruck 2004): Entwicklung und Bewertung neuer Ansätze in der Pflanzensoziologie unter besonderer Berücksichtigung der Vegetationsklassifikation. - Archiv naturwissenschafticher Dissertationen 14: 297 pp. Galunder, Nümbrecht.

\section{1}

Berg, C., Dengler, J., Abdank, A. (2001) [Eds.]: Die Pflanzengesellschaften Mecklenburg-Vorpommerns und ihre Gefährdung - Tabellenband. - 341 pp. Weissdorn, Jena.

darin:

Dengler, J., Berg, C.: Erläuterungen zum Aufbau der Tabellen. - pp. 9-14.

Dengler, J.: Polygono arenastri-Poetea annuae. - pp. 93-95.

Dengler, J.: Sisymbrietea. - pp. 96-103.

Dengler, J.: Koelerio-Corynephoretea. - pp. 118-136.

Dengler, J.: Festuco-Brometea. - pp. 137-143.

Dengler, J.: Trifolio-Geranietea. - pp. 159-177.

Dengler, J.: Artemisietea vulgaris. - pp. 178-210.

\section{III.3 Sonstige Publikationen mit peer review}

\section{Zur Veröffentlichung eingereicht}

Jansen, F., Dengler, J., Berg, C.: VegMV, the Vegetation Database of Mecklenburg-Vorpommern. - Manuskript für Biodiversity \& Ecology (im Review).

2010

Schmiedel, U., Dengler, J., Luther-Mosebach, J., Gröngröft, A., Muche, G., Petersen, A., Strohbach, B. J., Jürgens, N. (2010): Patterns and dynamics of vascular plant diversity along the BIOTA transects in southern Africa. - In: Schmiedel, U., Jürgens, N. [Hrsg.]: Biodiversity in Southern Africa. Volume 2: Patterns and Processes at Regional Scale: pp. 118-135. Klaus Hess Publishers, Göttingen \& Windhoek.

\section{9}

Mucina, L., Dengler, J., Bergmeier, E., Čarni, A., Dimopolous, P., Jahn, R., Matevski, V. (2009): New and validated high-rank syntaxa from Europe. - Lazaroa 30: 269-278. 
Ruprecht, E., Szabó, A., Enyedi, M. Z., Dengler, J. (2009): Steppe-like grasslands in Transylvania (Romania): characterisation and influence of management on species diversity and composition. - Tuexenia 29: 353368 + 1 Tabelle.

2008

Dengler, J., Boch, S. (2008): Forest-edge communities (Trifolio-Geranietea sanguinei) on the island of Saaremaa (Estonia): Phytosociology and biodiversity patterns. - In: Dengler, J., Dolnik, C., Trepel, M. [Eds.]: Flora, Vegetation und Naturschutz zwischen Schleswig-Holstein und Südamerika - Festschrift für Klaus Dierßen zum 60. Geburtstag. - Mitteilungen der Arbeitsgemeinschaft Geobotanik in SchleswigHolstein und Hamburg 65: 257-286 + 2 Beilagen. Kiel.

Dengler, J., Chytrý, M., Ewald, J. (2008): Phytosociology. - In: Jørgensen, S. E., Fath, B. D. [Eds.]: Encyclopedia of Ecology: pp. 2767-2779. Elsevier, Oxford.

Jansen, F., Dengler, J. (2008): GermanSL - Eine universelle taxonomische Referenzliste für Vegetationsdatenbanken in Deutschland. - Tuexenia 28: 239-253.

Löbel, S., Dengler, J. (2008) [,,2007”]: Dry grassland communities on southern Öland: phytosociology, ecology, and diversity. - In: van der Maarel, E. [Ed.]: Structure and dynamics of alvar vegetation on Öland and some related dry grasslands - Dedicated to Ejvind Rosén on his $65^{\text {th }}$ birthday. - Acta Phytogeographica Suecica 88: 13-32. Svenska Växtgeografiska Sällskapet, Uppsala.

2007

Dengler, J., Eisenberg, M., Schröder, J. (2007): Die grundwasserfernen Saumgesellschaften Nordostniedersachsens im europäischen Kontext - Teil II: Säume nährstoffreicher Standorte (Artemisietea vulgaris) und vergleichende Betrachtung der Saumgesellschaften insgesamt. - Tuexenia 27: 91-136 + 6 Tabellen.

2006

Boch, S., Dengler, J. (2006): Floristische und ökologische Charakterisierung sowie Phytodiversität der Trockenrasen auf der Insel Saaremaa (Estland). - In: Bültmann, H., Fartmann, T., Hasse, T. [Eds.]: Trockenrasen auf unterschiedlichen Betrachtungsebenen. - Arbeiten aus dem Institut für Landschaftsökologie Münster 15: 55-71. Münster.

Dengler, J. (2006): Variabilität von Artendichte und Artenzusammensetzung auf unterschiedlichen räumlichen Skalenebenen - Exemplarische Untersuchungen aus Trockenrasen und Konsequenzen für das Probedesign von Biodiversitätsuntersuchungen. - In: Bültmann, H., Fartmann, T., Hasse, T. [Eds.]: Trockenrasen auf unterschiedlichen Betrachtungsebenen. - Arbeiten aus dem Institut für Landschaftsökologie Münster 15: 73-81. Münster.

Dengler, J., Eisenberg, M., Schröder, J. (2006): Die grundwasserfernen Saumgesellschaften Nordostniedersachsens im europäischen Kontext - Teil I: Säume magerer Standorte (Trifolio-Geranietea sanguinei). Tuexenia 26: 51-93 + 9 Tabellen.

Dengler, J., Löbel, S., Boch, S. (2006): Dry grassland communities of shallow, skeletal soils (SedoScleranthenea) in northern Europe. - Tuexenia 26: 159-190 + 6 Tabellen.

Dengler, J., Rūsiņa, S., Boch, S., Bruun, H. H., Diekmann, M., Dierßen, K., Dolnik, C., Dupré, C., Golub, V. B., Grytnes, J.-A., Helm, A., Ingerpuu, N., Löbel, S., Pärtel, M., Rašomavičius, V., Tyler, G., Znamenskiy, S. R., Zobel, M. (2006): Working group on dry grasslands in the Nordic and Baltic region - Outline of the project and first results for the class Festuco-Brometea. - Annali di Botanica, N. S. 6: 73-100. 
Friedel, A., Oheimb, G. von, Dengler, J., Härdtle, W. (2006): Species diversity and species composition of epiphytic bryophytes and lichens - a comparison or managed and unmanaged beech forests in NE Germany. - Feddes Repertorium 117: 172-185.

Timmermann, T., Dengler, J., Abdank, A., Berg, C. (2006): Objektivierung von Naturschutzbewertungen Das Beispiel Roter Listen von Pflanzengesellschaften. - Naturschutz und Landschaftsplanung 38: 133-139.

2005

Berg, C., Dengler, J. (2005): Moose und Flechten als diagnostische Arten von Pflanzengesellschaften - eine Übersicht aus Mecklenburg-Vorpommern. - Herzogia 18: 145-161.

Dengler, J. (2005): Zwischen Estland und Portugal - Gemeinsamkeiten und Unterschiede der Phytodiversitätsmuster europäischer Trockenrasen. - Tuexenia 25: 387-405.

Dengler, J., Berg, C., Jansen, F. (2005): New ideas for modern phytosociological monographs. - Annali di Botanica N. S. 5: 49-66.

2004

Berg, C., Dengler, J. (2004): Von der Datenbank zur Regionalmonografie - Erfahrungen aus dem Projekt „Die Pflanzengesellschaften Mecklenburg-Vorpommerns und ihre Gefährdung“. - Berichte der Reinhold-TüxenGesellschaft 16: 29-56.

Dengler, J., Koska, I., Timmermann, T., Berg, C., Clausnitzer, U., Isermann, M., Linke, C., Päzolt, J., Polte, T., Spangenberg, A. (2004): New descriptions and typifications of syntaxa within the project 'Plant communities of Mecklenburg-Vorpommern and their vulnerability' - Part II. - Feddes Repertorium 115: 343-392.

Marquardt, B., Rathjen, J., Boch, S., Kaiser, T., Dengler, J. (2004): Naturschutz im Industriegebiet? - Flora, Vegetation und Naturschutzpotenzial des Lüneburger Hafens. - Drosera 2004: 43-92.

2003

Dengler, J., Berg, C., Eisenberg, M., Isermann, M., Jansen, F., Koska, I., Löbel, S., Manthey, M., Päzolt, J., Spangenberg, A., Timmermann, T., Wollert, H. (2003): New descriptions and typifications of syntaxa within the project 'Plant communities of Mecklenburg-Vorpommern and their vulnerability' - Part I. - Feddes Repertorium 114: 587-631.

Dengler, J., Krebs, J. (2003): Zwei neue Saumassoziationen der Klasse Trifolio-Geranietea sanguinei aus dem norddeutschen Tiefland. - Drosera 2003: 11-32.

Kießlich, M., Dengler, J., Berg, C. (2003): Die Gesellschaften der Bidentetea tripartitae Tx. \& al. ex von Rochow 1951 in Mecklenburg-Vorpommern mit Anmerkungen zur Synsystematik und Nomenklatur der Klasse. - Feddes Repertorium 114: 91-139.

\section{2}

Dengler, J., Eisenberg, M., Kraft, A., Löbel, S. (2002): Die Ilmenauniederung „Düvelsbrook“ - Standort, Vegetation und Naturschutz eines Feuchtwiesengebietes bei Lüneburg. - Drosera 2002: 33-78.

2001

Berg, C., Timmermann, T., Dengler, J. (2001): Methodische Ansätze für eine „Rote Liste der Pflanzengesellschaften Mecklenburg-Vorpommerns“: Naturschutzfachliche Wertstufe. - Berichte der Reinhold-Tüxen-Gesellschaft 13: $217-221$. 
Dengler, J. (2001): Erstellung und Interpretation synchorologischer Karten am Beispiel der Klasse KoelerioCorynephoretea. - Berichte der Reinhold-Tüxen-Gesellschaft 13: 223-228.

\section{0}

Dengler, J., Lemke, H., Wollert, H. (2000): Zwei Stipa-Arten im Uecker-Randow-Gebiet (MecklenburgVorpommern) wiederentdeckt - Mit Anmerkungen zum Stipa pennata-Aggregat in Nordostdeutschland. - Gleditschia 28: 17-26.

\section{9}

Kratzert, G., Dengler, J. (1999): Die Trockenrasen der „Gabower Hänge“ am Oderbruch. - Verhandlungen des Botanischen Vereins von Berlin und Brandenburg 132: 285-329 + 10 Tabellen + 1 Karte.

Wolfram, C., Dengler, J. (1999): Bryum torquescens Bruch \& Schimp. neu für Brandenburg, mit Hinweisen zur Bestimmung und Verbreitung der Art. - Verhandlungen des Botanischen Vereins von Berlin und Brandenburg 132: $363-370$.

\section{8}

Dengler, J. (1998): Zur Unterscheidung von Phleum pratense s. str. und Phleum bertolonii. - Floristische Rundbriefe 32: 7-12.

\section{7}

Dengler, J. (1997): Gedanken zur synsystematischen Arbeitsweise und zur Gliederung der Ruderalgesellschaften (Artemisietea vulgaris s. 1.). Mit der Beschreibung des Elymo-Rubetum caesii ass. nova. - Tuexenia 17: 251-282 +4 Tabellen.

\section{6}

Dengler, J. (1996): Das Festuca ovina-Aggregat in Brandenburg und Berlin - Bestimmungsschlüssel und Kartieraufruf. - Verhandlungen des Botanischen Vereins von Berlin und Brandenburg 129: 133-139.

\section{5}

Dengler, J. (1995): Anmerkungen zur Bestimmung der brandenburgischen Vertreter von Vicia sect. Cracca. - Verhandlungen des Botanischen Vereins von Berlin und Brandenburg 128: 147-154.

Dengler, J. (1995): Vier bislang wenig beachtete Gefäßpflanzensippen der brandenburgischen Flora - Arabis sagittata (Bertol.) DC., Centaurea jacea L. ssp. subjacea (Beck) Hyl., Centaurea nigrescens Willd. und Vicia tenuissima (Bieb.) Schinz \& Thell. - Verhandlungen des Botanischen Vereins von Berlin und Brandenburg 128: 131145.

\section{4}

Dengler, J. (1994): Flora und Vegetation von Trockenrasen und verwandten Gesellschaften im Biosphärenreservat Schorfheide-Chorin. - Gleditschia 22: 179-321. 


\section{III.4 Sonstige Publikationen ohne peer review}

\section{0}

Dengler, J., GIVD Steering Committee (2010): GIVD, a new ecological metadatabase. - Frontiers of Biogeography 2: 70.

Ewald, J., Finckh, M., Dengler, J. (2010): Bericht von der 9. internationalen Tagung zu Vegetationsdatenbanken mit dem Schwerpunkt „Klimawandel“ in Hamburg. - Tuexenia 30.: 489-492.

Jandt, U., Becker, T., Dengler, J., Janišová, M. (2010): Dry grasslands: species interactions and distribution Editorial to the Special Feature with contributions from the 6th European Dry Grassland Meeting 2009 in Halle (Saale). - Tuexenia 30: 349-355.

\section{9}

Dengler, J., Ruprecht, E., Szabó, A., Turtureanu, D., Beldean, M., Uğurlu, E., Pedashenko, H., Dolnik, C., Jones, A. (2009): EDGG cooperation on syntaxonomy and biodiversity of Festuco-Brometea communities in Transylvania (Romania): report and preliminary results. - Bulletin of the European Dry Grassland Group 4: 13-19.

Dolnik, C., Dengler, J., Jandt, U., Kiehl, K.: Dry Grasslands in a Changing Environment - Special Feature with contributions from the $5^{\text {th }}$ Dry Grassland Meeting 2008 in Kiel. - Tuexenia 29: 331-337.

\section{8}

Dengler, J. (2008): Veröffentlichungsverzeichnis von Klaus Dierßen. - In: Dengler, J., Dolnik, C., Trepel, M. [Eds.]: Flora, Vegetation und Naturschutz zwischen Schleswig-Holstein und Südamerika - Festschrift für Klaus Dierßen zum 60. Geburtstag. - Mitteilungen der Arbeitsgemeinschaft Geobotanik in Schleswig-Holstein und Hamburg 65: 31-44. Kiel.

Dengler, J., Allers, M.-A. (2008): Varying diversity patterns of different plant groups at different spatial scales in central European landscapes. - In: Mucina, L., Kalwij, J. M., Smith, V. R., Chytrý, M., White, P. S., Cilliers, S. S., Pillar, V. D., Zobel, M., Sun, I.-F. [Eds.]: Frontiers of Vegetation Science - an Evolutionary Angel: pp. 4042. Keith Phillips Images, Somerset West.

Dengler, J., Rūsiņa, S., Boch, S., Löbel, S. (2008): The basiphilous semi-dry grasslands (Festuco-Brometea) in N and NE Europe: gradient analysis and large-scale classification. - In: Mucina, L., Kalwij, J. M., Smith, V. R., Chytrý, M., White, P. S., Cilliers, S. S., Pillar, V. D., Zobel, M., Sun, I.-F. [Eds.]: Frontiers of Vegetation Science - an Evolutionary Angel: pp. 42-43. Keith Phillips Images, Somerset West.

Kiehl, K., Albrecht, H., Röder, D., Jeschke, M., Dolnik, C., Schwabe, A., Jandt, U., Dengler, J. (2008): Wiederherstellung und spontane Ansiedlung von Trocken- und Halbtrockenrasen in ursprünglichen und urbanindustriellen Lebensräumen - Tagung in Freising-Weihenstephan vom 6. bis 8. September 2007. - Tuexenia 28: 263-266.

Schmiedel, U., Dengler, J., Etzold, S. (2008): Population dynamics on quartz fields in South Africa - climate change effects on conservation efforts. - In: Mucina, L., Kalwij, J. M., Smith, V. R., Chytrý, M., White, P. S., Cilliers, S. S., Pillar, V. D., Zobel, M., Sun, I.-F. [Eds.]: Frontiers of Vegetation Science - an Evolutionary Angel: pp. 163-165. Keith Phillips Images, Somerset West.

Trepel, M., Dolnik, C., Dengler, J. (2008): Flora, Vegetation und Naturschutz zwischen Schleswig-Holstein und Südamerika - Einführung in die Festschrift für Klaus Dierßen zum 60. Geburtstag [dt. und engl.]. - In: Dengler, J., Dolnik, C., Trepel, M. [Eds.]: Flora, Vegetation und Naturschutz zwischen Schleswig-Holstein und 
Südamerika - Festschrift für Klaus Dierßen zum 60. Geburtstag. - Mitteilungen der Arbeitsgemeinschaft Geobotanik in Schleswig-Holstein und Hamburg 65: 7-10. Kiel.

\section{7}

Allers, M.-A., Dengler, J. (2007): Small-scale patterns of plant species richness in the central European landscape. - Verhandlungen der Gesellschaft für Ökologie 37: 181.

Jeschke, M., Dengler, J., Boch, S., Dolnik, C., Kiehl, K., Löbel, S. (2007): Species-area relationships in European dry grasslands - A comparative analysis across regions, taxa, and scales. - Verhandlungen der Gesellschaft für Ökologie 37: 180.

\section{6}

Bültmann, H., Hasse, T., Dörsing, M., Jandt, U., Becker, T., Dengler, J. (2006): Trockenrasen auf unterschiedlichen Betrachtungsebenen - Zweite Jahrestagung der AG Trockenrasen vom 26.-28.08.05 in Münster. - Tuexenia 26: 389-390.

Dengler, J. (2006): Polycarpon tetraphyllum (L.) L. - In: Romahn, K. [Ed.]: Funde seltener, gefährdeter, neuer und wenig beachteter Gefäßpflanzen in Schleswig-Holstein. - Kieler Notizen zur Pflanzenkunde 34: 57-58.

Dengler, J. (2006): Xerothermvegetation in NO-Niedersachsen und im Mittleren Elbetal. - In: Härdtle, W., Horst, K., Prüter, J. [Eds.]: Flora und Vegetation im nordöstlichen Niedersachsen. - Jahrbuch des Naturwisschenschaftlichen Vereins für das Fürstentum Lüneburg von 1851 e. V., Sonderheft 1: 107-114.

Dengler, J. (2006): Zweizahn-Gesellschaften und Melden-Uferfluren (Bidentetea) im Elbtal. - In: Härdtle, W., Horst, K., Prüter, J. [Eds.]: Flora und Vegetation im nordöstlichen Niedersachsen. - Jahrbuch des Naturwisschenschaftlichen Vereins für das Fürstentum Lüneburg von 1851 e. V., Sonderheft 1: 115-116.

Dengler, J., Allers, M.-A. (2006): Plant species richness of the central European landscape on different spatial scales measured with a new approach. - Verhandlungen der Gesellschaft für Ökologie 36: 159.

Dengler, J., Boch, S. (2006): Vegetation des NSG Kalkberg in Lüneburg. - In: Härdtle, W., Horst, K., Prüter, J. [Eds.]: Flora und Vegetation im nordöstlichen Niedersachsen. - Jahrbuch des Naturwisschenschaftlichen Vereins für das Fürstentum Lüneburg von 1851 e. V., Sonderheft 1: 49-55.

Dengler, J., Fischer, P., Härdtle, W. (2006): Flusslandschaft Elbe zwischen Radegast und Dömitz (Elbeexkursion 1). - In: Härdtle, W., Horst, K., Prüter, J. [Eds.]: Flora und Vegetation im nordöstlichen Niedersachsen. - Jahrbuch des Naturwisschenschaftlichen Vereins für das Fürstentum Lüneburg von 1851 e. V., Sonderheft 1: 69-77.

\section{5}

Boch, S., Dengler, J. (2005): Patterns of plant species richness of dry grasslands on the island of Saaremaa (Estonia). - Verhandlungen der Gesellschaft für Ökologie 35: 203.

Dengler, J., Jandt, U. (2005): Arbeitsgruppe „Trockenrasen“ gegründet - Bericht von der ersten Jahrestagung unter dem Motto „Trockenrasen als Biodiversitätshotspots“. - Tuexenia 25: 375-378.

Dengler, J., Koperski, M., Boch, S., Marquardt, B., Rost, S. (2005) [„,2004“]: Zur Flora des NSG Kalkberg in Lüneburg unter besonderer Berücksichtigung seiner Moose. - Jahrbuch des Naturwisschenschaftlichen Vereins für das Fürstentum Lüneburg von 1851 e. V. 43: 175-187. 


\section{4}

Bedall, P., Bruchmann, I., Gascho, C., Hoeft, I., Maroschek, N., Dengler, J. (2004): Artenzusammensetzung und Phytodiversität in Kalkmagerrasen entlang eines Höhentransektes am Südalpenrand (Italien, Trentino). - Kieler Notizen zur Pflanzenkunde Schleswig-Holstein und Hamburg 32: 44-49.

Boch, S., Dengler, J. (2004): Die Trockenrasen der Insel Saaremaa (Estland): Charakterisierung und Phytodiversität (erste Ergebnisse). - Kieler Notizen zur Pflanzenkunde Schleswig-Holstein und Hamburg 32: 3-8.

Dengler, J. (2004): Artenzusammensetzung und Phytodiversität von Trockenrasen auf Granitgrus entlang eines Höhentransektes in der Serra da Estrela (Portugal). - Kieler Notizen zur Pflanzenkunde Schleswig-Holstein und Hamburg 32: 49-56.

Dengler, J. (2004): Phytodiversitätsmuster in nordostdeutschen Trockenrasen. - Kieler Notizen zur Pflanzenkunde Schleswig-Holstein und Hamburg 32: 14-19.

Dengler, J., Bedall, P., Bruchmann, I., Hoeft, I., Lang, A. (2004): Artenzahl-Areal-Beziehungen in uckermärkischen Trockenrasen unter Berücksichtigung von Kleinstflächen - eine neue Methode und erste Ergebnisse. - Kieler Notizen zur Pflanzenkunde Schleswig-Holstein und Hamburg 32: 20-25.

Dengler, J., Jandt, U. (2004) [Eds.]: Trockenrasen als Biodiversitätshotspots - Erste Jahrestagung der Arbeitsgruppe „Trockenrasen“. - Kieler Notizen zur Pflanzenkunde Schleswig-Holstein und Hamburg 32: 1-56.

Dolnik, C., Behmann, H., Dengler, J., Horst, E., Jansen, W., Kempe, W., Kieckbusch, J., Kunzmann, D., Lütt, S., Putfarken, D., Romahn, K., Stolley, G., Timmermann-Trosiener, I. (2004): Funde von seltenen, gefährdeten und wenig beachteten Gefäßpflanzen in Schleswig-Holstein. - Kieler Notizen zur Pflanzenkunde Schleswig-Holstein und Hamburg 32: 103-123.

Drews, H., Dengler, J. (2004): Steilufer an der nordoldenburgischen Küste: Artenausstattung, Vegetation und Pflegekonzept unter besonderer Berücksichtigung der Kalkhalbtrockenrasen und der wärmeliebenden Säume. - Kieler Notizen zur Pflanzenkunde Schleswig-Holstein und Hamburg 32: 57-95.

Eisenberg, M., Dengler, J., Hobohm, C., Härdtle, W. (2003): Plant species diversity in skirt communities: Investigations from Wendland / Lower Saxony. - Verhandlungen der Gesellschaft für Ökologie 33: 80.

Löbel, S., Dengler, J., Hobohm, C. (2004): Beziehungen zwischen der Artenvielfalt von Gefäßpflanzen, Moosen und Flechten in Trockenrasen der Insel Öland (Schweden). - Kieler Notizen zur Pflanzenkunde in Schleswig-Holstein und Hamburg 32: 9-13.

\section{2}

Abdank, A., Berg, C., Dengler, J. (2002) [,2000“]: Gefährdungseinstufung von Pflanzengesellschaften - Vorgehen bei der „Roten Liste der Pflanzengesellschaften von Mecklenburg-Vorpommern“. - In: Rennwald, E. [Ed.]: Verzeichnis und Rote Liste der Pflanzengesellschaften Deutschlands - mit Datenservice auf CD-ROM. - Schriftenreihe für Vegetationskunde 35: 49-63. Bundesamt für Naturschutz, Bonn.

Dengler, J. (2002): Beiträge zur Nomenklatur einiger Ruderalgesellschaften. - In: Rennwald, E. [Ed.]: Verzeichnis und Rote Liste der Pflanzengesellschaften Deutschlands - mit Datenservice auf CD-ROM. - Schriftenreihe für Vegetationskunde 35: 65-69. Bundesamt für Naturschutz, Bonn.

Dengler, J., Berg, C. (2002) [,„2000“]: Klassifikation und Benennung von Pflanzengesellschaften - Ansätze zu einer konsistenten Methodik im Rahmen des Projekts „Rote Liste der Pflanzengesellschaften von MecklenburgVorpommern“. - In: Rennwald, E. [Ed.]: Verzeichnis und Rote Liste der Pflanzengesellschaften Deutschlands mit Datenservice auf CD-ROM. - Schriftenreihe für Vegetationskunde 35: 17-47. Bundesamt für Naturschutz, Bonn. 
Löbel, S., Dengler, J., Hobohm, C. (2002): Patterns of phytodiversity in dry grasslands on Öland (Sweden). - Verhandlungen der Gesellschaft für Ökologie 32: 52

Rennwald, E. unter Mitarbeit von zahlreichen Autoren, darunter Dengler, J. (2002) [,2000“]: Rote Liste der Pflanzengesellschaften Deutschlands mit Anmerkungen zur Gefährdung. - In: Rennwald, E. [Ed.]: Verzeichnis und Rote Liste der Pflanzengesellschaften Deutschlands - mit Datenservice auf CD-ROM. - Schriftenreihe für Vegetationskunde 35: 393-592. Bundesamt für Naturschutz, Bonn.

Rennwald, E. unter Mitarbeit von zahlreichen Autoren, darunter Dengler, J. (2002) [,2000“]: Verzeichnis der Pflanzengesellschaften Deutschlands mit Synonymen und Formationseinteilung. - In: Rennwald, E. [Ed.]: Verzeichnis und Rote Liste der Pflanzengesellschaften Deutschlands - mit Datenservice auf CD-ROM. - Schriftenreihe für Vegetationskunde 35: 121-391. Bundesamt für Naturschutz, Bonn.

\section{1}

Dengler, J. (2001): Uneven distribution of phytodiversity in NE German dry grassland communities. - Verhandlungen der Gesellschaft für Ökologie 31: 28.

Dengler, J., Löbel, S., Michl, T. (2001): Die Steinhöhe - ein ökologisches Kleinod in Lüneburg (Ergebnisse des vegetationskundlichen Studentenpraktikums im Sommersemester 1999). - Jahrbuch des Naturwisschenschaftlichen Vereins für das Fürstentum Lüneburg von 1851 e. V. 42: 143-188 + 1 Tabelle + 1 Karte.

Dengler, J., Siemsen, M., Wolfram, C., Berg, C., Drews, H., Keienburg, T., Lütt, S., Martin, C., Schröder, W. (2001): Neue Funde gefährdeter und anderer bemerkenswerter Moose in Schleswig-Holstein. - Kieler Notizen zur Pflanzenkunde Schleswig-Holstein und Hamburg 27/28: 8-27.

Dengler, J., Wollert, H. (2001): Zum Auftreten des Vulpietum myuri in Mecklenburg und Nordostniedersachsen. Botanischer Rundbrief für Mecklenburg-Vorpommern 35: 97-100, 1 Tab.

Siemsen, M., Wolfram, C., Dengler, J. (2001): Neue Funde gefährdeter und anderer bemerkenswerter Moose in Schleswig-Holstein und Hamburg - 2. Folge. - Kieler Notizen zur Pflanzenkunde Schleswig-Holstein und Hamburg 27/28: 28-69.

\section{0}

Dengler, J. (2000): Synsystematische Stellung und Gliederung der uckermärkischen Sandtrockenrasen. - Berichte der Naturwissenschaftlichen Gesellschaft Bayreuth 24: 302-306.

\section{8}

Dengler, J. (1998): Bemerkenswerte Gefäßpflanzenfunde in der Xerothermvegetation von MecklenburgVorpommern - Teil I. - Botanischer Rundbrief für Mecklenburg-Vorpommern 32: 87-110.

Dengler, J. (1998): Der Geschützte Landschaftsbestandteil „Mühlenberg“ bei Brodowin - Flora, Vegetation und Bedeutung für den Naturschutz. - Naturschutz und Landschaftspflege in Brandenburg 7: 149-158.

Dengler, J. (1998): Neues von den schmalblättrigen Schwingel-Sippen (Festuca ovina agg. und F. rubra agg.) in Deutschland mit besonderer Berücksichtigung von Schleswig-Holstein und Hamburg. - Kieler Notizen zur Pflanzenkunde Schleswig-Holstein und Hamburg 25/26: 6-32.

Dengler, J., Hünert, C. (1998): Ein bemerkenswerter Trockenhang in Kiel-Elmschenhagen mit zwei für SchleswigHolstein neuen Gefäßpflanzenarten. - Kieler Notizen zur Pflanzenkunde Schleswig-Holstein und Hamburg 25/26: $80-87$. 


\section{6}

Dengler, J. (1996): Das Festuca ovina-Aggregat in Mecklenburg-Vorpommern - Bestimmungsschlüssel und Kartieraufruf. - Botanischer Rundbrief für Mecklenburg-Vorpommern 29: 89-96.

Dengler, J. (1996): Anmerkungen zur Taxonomie und Bestimmung von Schaf-Schwingeln i. w. S. (Festuca ovina agg.) in Deutschland mit besonderer Berücksichtigung Schleswig-Holsteins. - Kieler Notizen zur Pflanzenkunde Schleswig-Holstein und Hamburg 24: 1-29.

\section{III.5 Buchrezensionen}

Seit 1998 über 100 Besprechungen wissenschaftlicher Bücher in den Kieler Notizen zur Pflanzenkunde, in Feddes Repertorium, Phytocoenologia und im Bulletin of the European Dry Grassland Group, teilweise gemeinsam mit Co-Autoren. 


\section{Verzeichnis von Lehrveranstaltungen an Universitäten}

\section{IV.1 Doktorandenkurse}

Publizieren in internationalen ökologischen Journals („Paper-Writing-Seminar") für Diplomanden, Doktoranden und Postdocs (teilweise auf Englisch, wöchentlich) [Universität Hamburg, seit 2007]

Forschungs-, Publikations- und Karrierestrategien für NachwuchswissenschaftlerInnen in der Ökologie [Universität Hamburg, Sommersemester 2008]

Vegetation science and databanks: A contribution to biodiversity informatics. - Teil des Workshops „Techniques for quantifying plant diversity“ der DFG Graduate School 1086 (auf Englisch) [Universität Göttingen, Wintersemester 2005/06]

\section{IV.2 MSc.-Modul}

Ökologie terrestrischer Lebensräume (Mitveranstalter) [Universität Hamburg, seit 2009]

- Praktikum Ökologie terrestrischer Lebensräume (abwechselnd: Mittleres Elbetal und Nordseeküste)

- Seminar Ökologie von Biozönosen

\section{IV.3 BSc.-Module}

Vegetations-, boden- und landschaftsökologische Exkursion und Geländeübung (teilweise auf Englisch; Alleinveranstalter) [Universität Hamburg, seit 2010]

- Seminar „Ökologie und Umweltprobleme des Exkursionsgebietes“

- Ökologische Fortgeschrittenen-Exkursion mit Geländeübung (Uckermark)

Methoden der Vegetationsökologie und Biodiversitätsforschung (teilweise auf Englisch; Leitung) [Universität Hamburg, seit 2009/10]

- Vorlesung Statistische und raumbezogene Methoden der Vegetationsökologie und Biodiversitätsforschung

- Übung Analyse vegetationsökologischer Daten mittels statistischer Verfahren und geografischer Informationssysteme (GIS)

Biologie, Ökologie und Bestimmung von Moosen (Alleinveranstalter) [Universität Hamburg, seit 2009/10]

- Seminar Biologie der Moose

- Übung Bestimmung heimischer Moose

- Exkursionen zu Mooslebensräumen in Hamburg und Umgebung

Grundlagen der Biologie (Mitveranstalter) [Universität Hamburg, seit 2007]

- Vorlesung Grundlagen der Biologie

Biodiversität der Pflanzen (Mitveranstalter) [Universität Hamburg, 2009]

- Bestimmungsübungen höherer Pflanzen

Ökologie (Mitveranstalter) [Universität Hamburg, seit 2008]

- Vorlesung Allgemeine Ökologie

- Praktikum Ökologie 


\section{IV.4 Sonstige Vorlesungen}

Methoden der Vegetationsanalyse und Biodiversitätsforschung [Universität Hamburg, 2007/08]

Vegetation und Ökologie der Erde [Universität Hamburg, 2007/08]

Vegetation Europas [Universität Lüneburg, 1998/99]

\section{IV.5 Sonstige Übungen}

Methoden der Vegetationsanalyse und Biodiversitätsforschung (Statistik) [Universität Hamburg, 2007/08]

Wissenschaftliches Arbeiten in der Ökologie [Universität Lüneburg, 2006]

Einführung in die Biologie und Bestimmung der Moose [Universität Lüneburg, 1998-2006]

Botanische Bestimmungsübungen: Samenpflanzen [Universität Lüneburg, 1999-2005]

\section{IV.6 Sonstige Projektpraktika u. ä.}

Ökologisches Praktikum: Vegetationskundliche Methoden (als Projektpraktikum mit wechselnden Untersuchungsgebieten und Fragestellungen; jeweils bestehend aus vegetationskundlichfloristischer und bodenkundlicher Datenerhebung, naturwissenschaftlicher Auswertung, GISKarten-Erstellung und planerischer Umsetzung) [Universität Lüneburg]:

- Steinhöhe (ehemaliges militärisches Übungsgelände mit Wald- und Offenland) [Sommersemester 1999, in Zusammenarbeit mit dem Umweltinformatiker P. Mix]

- Wilschenbruch (überwiegend brach gefallene Feuchtwiesenniederung) [Sommersemester $2000+$ Wintersemester 2000/01]

- Naturschutz im Industriegebiet Lüneburger Hafen? [Sommersemester 2002 + Wintersemester 2002/03, gemeinsam mit dem Landschaftsarchitekten Dr. T. Kaiser]

Vegetationsökologische Geländeübungen in der Uckermark (mit Vorbereitungsseminar, Exkursions-, Projekt-, bodenkundlichem Labor- und Statistikteil) [Universität Lüneburg, Sommersemester 2004]

\section{IV.7 Sonstige Exkursionen}

Große Bioökologische Exkursion in die Uckermark [Universität Lüneburg, 2005]

Große Bioökologische Exkursion in die Südalpen [Mitwirkung, Universität Lüneburg, 2004]

Geobotanische Fortgeschrittenenexkursion in die Uckermark [Mitwirkung, Universität Kiel bzw. Lüneburg, 1994-2003]

Große Bioökologische Exkursion in die Schweizer Alpen (Davos) mit Geländeübungen [Universität Lüneburg, 2001]

Große Bioökologische Exkursion in die Serra da Estrela (Portugal) [Mitwirkung, Universität Lüneburg, 1999]

Geobotanische Fortgeschrittenenexkursion in den Harz und den Kyffhäuser [Mitwirkung, Universität Kiel, 1996] 
Geobotanische Fortgeschrittenenenexkursion auf die Lofoten [Mitwirkung, Universität Kiel, 1995]

\section{IV.8 Sonstige Seminare}

Biodiversität - eine ökologische Einführung [Universität Lüneburg, 2006]

Einführung in die Ökologie [Universität Lüneburg, 2006]

Geobotanisches Hauptseminar: Vegetationsökologische Methoden und Konzepte [Universität Lüneburg, 1999-2005]

Einführung in die Landschaftsökologie unter besonderer Berücksichtigung des norddeutschen Tieflandes [Universität Lüneburg, 2003-2005]

Ökologie und Umweltprobleme des Alpenraumes [Universität Lüneburg, 2000/01]

Seminar zur Großen Bioökologischen Exkursion in die Serra da Estrela (Portugal) [Mitwirkung, Universität Lüneburg, 1999]

\section{IV.9 Kolloquien}

Ökologisches Kolloquium [Mitwirkung, Universität Lüneburg, 1999-2005]

Fernweh-Kolloquium: Fremde Länder aus umweltwissenschaftlichem Blickwinkel gesehen [Universität Lüneburg, 1999-2003]

\section{IV.10 Betreuung von Dissertationen}

Turtureanu, P. D. (laufend): Plant species diversity of forest clearings from Trascău (Apuseni Mountains, Romania). Ph.D. thesis, Babeş-Bolyai University, Cluj-Napoca, Romania [Betreuung]

Akasbi, Z. (laufend): Effects of different grazing regimes on semi-arid ecosystems in southern Morocco. Doktorarbeit, Universität Hamburg [Betreuung].

\section{IV.11 Betreuung von Diplom-, Staatsexamens-, Bachelor- und Semesterarbeiten}

Wallenfang, J. (2011): Testing the existence of the small island effect. $-41+$ XXVIII pp. + CD-ROM. Bachelorarbeit im Studiengang Biologie, Universität Hamburg [Betreuung und Zweitgutachter].

Lampe, P. (2011): Standortbedingungen und Phytodiversität der Trittrasengesellschaften (Polygono-Poetea annuae) in Hamburg. - 48 pp. + CD-ROM. Bachelorarbeit im Studiengang Biologie, Universität Hamburg [Betreuung und Zweitgutachter].

Rahmlow, M. (2011): Klassifikation der Trittrasengesellschaften (Polygono-Poetea annuae) Hamburgs unter besonderer Berücksichtigung der Moose. - 44 pp. + CD-ROM. Bachelorarbeit im Studiengang Biologie, Universität Hamburg [Betreuung und Zweitgutachter]. 
Peters, J. (2010): Plant diversity patterns at different spatial scales in a semi-arid savanna ecosystem in central Namibia. - 64 + IX pp. + CD-ROM. Diplomarbeit im Studiengang Landschaftsökologie, Universität Greifswald [Betreuung und Zweitgutachter].

Dorendorf, J. U. (2010): Hierarchical classification of Nama-Karoo vegetation in the Keetmanshoop area, southcentral Namibia. - 46 + XIX pp. + CD-ROM. Diplomarbeit im Studiengang Biologie, Universität Hamburg [Betreuung].

Pellowski, M. (2009): Verteilung von Pflanzenarten und ihren funtionellen Merkmalen entlang von Beweidungsgradienten in Zentralnamibia. - V + 54 + XXVI pp. + CD-ROM., Staatsexamensarbeit im Studiengang, Universität Hamburg [Betreuung].

Weber, J. (2009): Einfluss von Niederschlag und Beweidung auf die Populationsdynamik von Pflanzenarten in der Sukkulenten Karoo (Südafrika). - VII + 44 + XXVIII pp. + CD-ROM., Staatsexamensarbeit im Studiengang Biologie, Universität Hamburg [Betreuung].

Luther-Mosebach, J. (2009): Hierarchical classification of the vegetation on the communal farmland of Soebatsfontein in Namaqualand, South Africa The role of grazing history and habitat conditions. -47 pp. + CDROM. Diplomarbeitim Studiengang Biologie, Universität Hamburg [Betreuung].

Röwer, I. U. (2009): Small-scale vegetation and biodiversity patterns of termitaria (heuweltjies) under different grazing pressures in the communal area of Soebatsfontein, South Africa. - 58 pp. + CD-ROM. Diplomarbeit im Studiengang Biologie, Universität Hamburg [Betreuung].

Bösing, B. M. (2009): Interactions between wildlife and vegetation under different grazing intensities and habitat types in the Knersvlakte, South Africa. - IV + $44+$ XIII pp. + CD-ROM. Diplomarbeit im Studiengang Biologie, Universität Hamburg [Betreuung].

Haarmeyer, D. H. (2009): Effects of domestic livestock on the vegetation of the Knersvlakte, South Africa. - VI + $56+$ XV pp. + CD-ROM. Diplomarbeit im Studiengang Biologie, Universität Hamburg [Betreuung]. - Diese Arbeit wurde mit dem Preis der Gesellschaft für Ökologie (GfÖ) 2010 für die beste Diplomarbeit in Deutschland, Österreich und der Scchweiz, ausgezeichnet.

Schaare-Schlüterhof, O. (2008): Ökologische Studien zur biotischen und abiotischen Heterogenität sowie zu den Vegetationsmustern auf den namibischen BIOTA-Observatorien Narais/Duruchaus und Gellap-Ost/Nabaos. 71 S. + CD-ROM, Staatsexamensarbeit im Studiengang Biologie, Universität Hamburg [Betreuung].

Allers, M.-A. (2007): Muster des Pflanzenartenreichtums auf unterschiedlichen räumlichen Skalenebenen am Beispiel des Messtischblattes 2728 / Lüneburg. - VIII + 80 + III pp. + Anhang. Diplomarbeit im Fach Ökologie, Studiengang Umweltwissenschaften, Universität Lüneburg [Betreuung und Erstgutachter].

Boch, S. (2005): Die Trockenrasen der Insel Saaremaa (Estland): Charakerisierung - Standort - Syntaxonomie Biodiversität. - $89+$ IX pp. + Beilagemappe. Diplomarbeit im Fach Ökologie, Studiengang Umweltwissenschaften, Universität Lüneburg [Betreuung und Erstgutachter].

Eisenberg, M. (2003): Saumgesellschaften NO-Niedersachsens - Soziologie und Pflanzenartenvielfalt. - $112+$ X pp. + 5 Tabellen. Diplomarbeit im Fach Ökologie, Studiengang Umweltwissenschaften, Universität Lüneburg [Betreuung und Zweitgutachter].

Krebs, J. (2003): Vegetation und Naturschutz von Wald- und Gebüschsäumen in der Umgebung von Lüneburg. $102+8$ pp. + Beilagemappe. Diplomarbeit im Fach Ökologie, Studiengang Umweltwissenschaften, Universität Lüneburg [Betreuung und Zweitgutachter]. 
Löbel, S. (2002): Trockenrasen auf Öland: Syntaxonomie - Ökologie - Biodiversität. - $178+$ XIV pp. + 4 Tabellen. Diplomarbeit im Fach Ökologie, Studiengang Umweltwissenschaften, Universität Lüneburg [Betreuung und Zweitgutachter].

Kießlich, M. (2001): Vegetationskundliche Untersuchungen von Zweizahn-Gesellschaften (Bidentetea tripartitae) in Mecklenburg-Vorpommern. $-82+9$ pp. +8 Tabellen. Diplomarbeit im Fach Ökologie, Studiengang Umweltwissenschaften, Universität Lüneburg [Betreuung].

Kratzert, G. (1998): Die Trockenrasen der Gabower Hänge am Oderbruch. - 104 pp. + Anhang. Diplomarbeit im Studiengang Biologie, Universität Kiel [Betreuung].

Dormann, C. (1997): Sandrohr (Calamagrostis epigejos (L.) Roth) in Trockenrasen des Biosphärenreservates Schorfheide-Chorin: Bestandsstruktur, ökologische Auswirkungen und Pflegemaßnahmen. - Zeitschrift für Ökologie und Naturschutz 6: 207-217. - Semesterarbeit im Studiengang Biologie, Universität Kiel [Betreuung]. 